DOI 10.4171/JEMS/356

François Germinet · Abel Klein

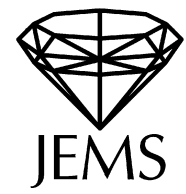

\title{
A comprehensive proof of localization for continuous Anderson models with singular random potentials
}

\author{
Received April 28, 2011
}

\begin{abstract}
We study continuous Anderson Hamiltonians with non-degenerate single site probability distribution of bounded support, without any regularity condition on the single site probability distribution. We prove the existence of a strong form of localization at the bottom of the spectrum, which includes Anderson localization (pure point spectrum with exponentially decaying eigenfunctions) with finite multiplicity of eigenvalues, dynamical localization (no spreading of wave packets under the time evolution), decay of eigenfunctions correlations, and decay of the Fermi projections. We also prove log-Hölder continuity of the integrated density of states at the bottom of the spectrum.
\end{abstract}

Keywords. Anderson localization, dynamical localization, random Schrödinger operator, continuous Anderson model, integrated density of states

\section{Contents}

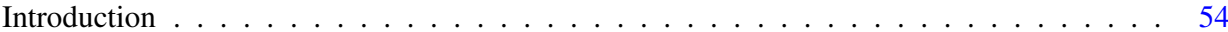

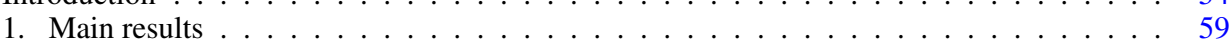

2. Anderson Hamiltonians . . . . . . . . . . . . . . . . . . . . . . . . . . 64

2.1. Normalized Anderson Hamiltonians _. . . . . . . . . . . . . . . . . . . . . . . . . 64

2.2. Generalized Anderson Hamiltonians . . . . . . . . . . . . . . . . . . . . 65

2.3. Finite volume Anderson Hamiltonians _ . . . . . . . . . . . . . . . . . . . . 66

2.4. Generalized eigenfunctions . . . . . . . . . . . . . . . . . . 67

2.5. Properties of finite volume operators $\ldots \ldots \ldots \ldots \ldots$

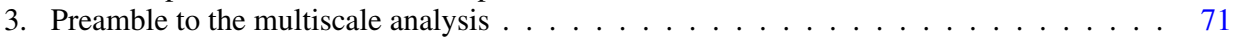

3.1. Good boxes and free sites . . . . . . . . . . . . . . . . . . . . . . 71

3.2. Tools for the multiscale analysis . . . . . . . . . . . . . . . . . 74

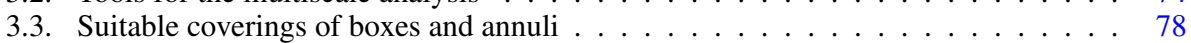

4. The multiscale analysis with a Wegner estimate . . . . . . . . . . . . 81

4.1. A priori finite volume estimates . . . . . . . . . . . . . . . . 81

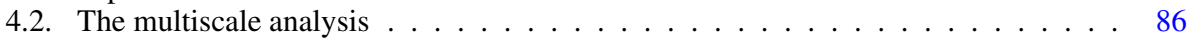

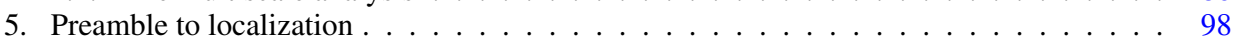

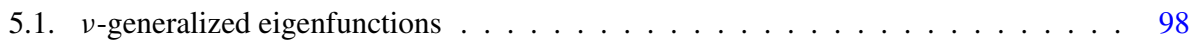

F. Germinet: Département de Mathématiques, Université de Cergy-Pontoise,

IUF, UMR 8088 CNRS, F-95000 Cergy-Pontoise, France; e-mail: francois.germinet@u-cergy.fr

A. Klein: Department of Mathematics, University of California, Irvine,

Irvine, CA 92697-3875, USA; e-mail: aklein@ uci.edu

Mathematics Subject Classification (2010): Primary 82B44; Secondary 47B80, 60H25, 81Q10 
5.2. Generalized eigenfunctions and good boxes . . . . . . . . . . . . . . . . . . . . 99

5.3. Generalized eigenfunctions and annuli of good boxes . . . . . . . . . . . 100

5.4. Generalized eigenfunction expansion . . . . . . . . . . . . . . . . . . . . . . . 101

5.5. Connection with point spectrum . . . . . . . . . . . . . . . . . 102

6. From the multiscale analysis to localization . . . . . . . . . . . . . . . . . . 103

6.1. The first spectral reduction . . . . . . . . . . . . . . . . . . . . . . 104

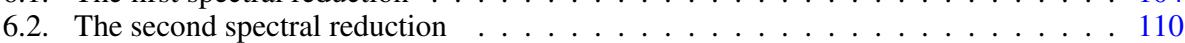

6.3. Annuli of good boxes . . . . . . . . . . . . . . . . . . . 113

7. Localization . . . . . . . . . . . . . . . . . . . . . . . . . 116

7.1. Anderson localization and finite multiplicity of eigenvalues . . . . . . . . . 116

7.2. Eigenfunctions correlations and dynamical localization . . . . . . . . . . . . . 117

7.3. Localization in expectation . . . . . . . . . . . . . . . . . . . . 122

8. Log-Hölder continuity of the integrated density of states . . . . . . . . . . . . 125

Appendix . . . . . . . . . . . . . . . . . . . . . . 128

A.1. The quantitative unique continuation principle . . . . . . . . . . . . . . . . 129

A.2. Application to Schrödinger operators with periodic potentials . . . . . . . . . . 135

References . . . . . . . . . . . . . . . . . . . . . . 138

\section{Introduction}

Anderson Hamiltonians are alloy-type random Schrödinger operators on $\mathrm{L}^{2}\left(\mathbb{R}^{d}\right)$ that model the motion of an electron moving in a randomly disordered crystal. They are the continuous analogue of the Anderson model, a random Schrödinger operator on $\ell^{2}\left(\mathbb{Z}^{d}\right)$.

In this paper we prove a strong form of localization at the bottom of the spectrum for Anderson Hamiltonians with a non-degenerate single site probability distribution with compact support, without any regularity condition on the single site probability distribution. This strong form of localization includes Anderson localization (pure point spectrum with exponentially decaying eigenfunctions) with finite multiplicity of eigenvalues, dynamical localization (no spreading of wave packets under the time evolution), decay of eigenfunctions correlations, and decay of the Fermi projections. We also prove log-Hölder continuity of the integrated density of states at the bottom of the spectrum.

Localization for random Schrödinger operators was first established in the celebrated paper by Gol'dsheid, Molchanov and Pastur [GoMP] for a certain one-dimensional continuous random Schrödinger operator. Localization is by now well established for one and quasi-one random Schrödinger operators [KuS, L, KIMP, CKM, KILS, Sto, DSS].

In the multi-dimensional case there is a wealth of results concerning localization for the (discrete) Anderson model and the (continuous) Anderson Hamiltonian as long as the single site probability distribution has enough regularity (absolutely continuous with a bounded density, Hölder continuous, log-Hölder continuous). In this case Anderson and dynamical localizations are well established: see, e.g., [FrS, MS1, FrMSS, DelLS, SiW, SVW, Dr, DrK1, Sp, DrK2, AM, K11, FK1, A, ASFH, W2, Klo4, HolM, CoH1, Klo2, GDB, FK2, KiSS1, KiSS2, DS, GK1, GK3, GK4, AENSS, K12]. Localization is also known in a random displacement model where the displacement probability distribution has a bounded density [Klo1, GhK, KloLNS], for a class of Gaussian random potentials [FiLM, U, LeMW], and for Poisson models where the single-site potentials are multiplied by random variables with bounded densities [MS2, CoH1]. What all these results have 
in common is the availability of random variables with sufficiently regular probability distributions, which can be exploited, in an averaging procedure, to produce an a priori Wegner estimate at all scales (an estimate on the probability of energy resonances in finite volumes); see, e.g., [We, FrS, HolM, CKM, CoH1, Klo2, CoHM, Ki, FiLM, St, CoHN, CoHKN, CoHK1, CoHK2].

In contrast, for the most natural random Schrödinger operators on the continuum (cf. [LiGP, Subsection 1.1]), the Bernoulli-Anderson Hamiltonian (simplest disordered substitutional alloy) and the Poisson Hamiltonian (simplest disordered amorphous medium), localization results in two or more dimensions were much harder to obtain. The Bernoulli -Anderson Hamiltonian is an Anderson Hamiltonian where the single site probability distribution is the distribution of a Bernoulli random variable, and the Poisson Hamiltonian is a random Schrödinger operator corresponding to identical impurities placed at locations given by a homogeneous Poisson point process on $\mathbb{R}^{d}$. In both cases the random variables with regular probability distributions are not available, so there is no a priori Wegner estimate.

Bourgain and Kenig [BoK] proved Anderson localization at the bottom of the spectrum for the Bernoulli-Anderson Hamiltonian. In their remarkable paper the Wegner estimate is established by a multiscale analysis using "free sites" and a new quantitative version of the unique continuation principle which gives a lower bound on eigenfunctions. Since this Wegner estimate has weak probability estimates and the underlying random variables are discrete, they also introduced a new method to prove Anderson localization from estimates on the finite-volume resolvents given by a single energy multiscale analysis. The new method does not use spectral averaging as in [DelLS, SiW, CoH1], which requires random variables with bounded densities. It is also not an energy-interval multiscale analysis as in [FrMSS, DrK1, FK2, GK1, K12], which requires better probability estimates.

Germinet, Hislop and Klein [GHK1, GHK2, GHK3] established Anderson localization at the bottom of the spectrum for the Poisson Hamiltonian, using a multiscale analysis that exploits the probabilistic properties of Poisson point processes to control the randomness of the configurations, and at the same time allows the use of the new ideas introduced by Bourgain and Kenig.

Aizenman, Germinet, Klein, and Warzel [AGKW] used a Bernoulli decomposition for random variables to show that spectral localization (pure point spectrum with probability one) for Anderson Hamiltonians follows from an extension of the Bourgain-Kenig results to nonhomogeneous Bernoulli-Anderson Hamiltonians, which incorporate an additional background potential and allow the variances of the Bernoulli terms not to be identical but only uniformly positive. Such random Schrödinger operators are generalized Anderson Hamiltonians as in Definition 2.2, for which we prove Anderson and dynamical localization in this paper, thus providing a proof of the required extension stated in [AGKW, Theorem 1.4].

In this article we provide a comprehensive proof of localization for Anderson Hamiltonians, drawing on the methods of [FrS, FrMSS, DrK1, CoH1, FK2, GK1, GK6, K12] and incorporating the new ideas of $[\mathrm{BoK}]$. We make no assumptions on the single site probability distribution except for compact support. (The proof can be extended to dis- 
tributions of unbounded support with appropriate assumptions on the tails of the distribution.) We perform a multiscale analysis to obtain probabilistic statements about restrictions of the Anderson Hamiltonian to finite volumes. From the conclusions of the multiscale analysis we extract an infinite volume characterization of localization: a probabilistic statement concerning the generalized eigenfunctions of the (infinite volume) Anderson Hamiltonian, from which we derive both Anderson and dynamical localization, as well as other consequences of localization, such as decay of eigenfunctions correlations (e.g., SULE, SUDEC) and decay of the Fermi projections.

This new infinite volume description of localization (given in Theorem 1.2(B)) yields all the manifestations of localization that have been previously derived from the energy interval multiscale analysis for sufficiently regular single site probability distribution [FrMSS, DrK1, GDB, DS, GK1, GK6, K12]. This description may also be derived from the energy interval multiscale analysis (see Remark 1.7); it is implicit in [GK6]. One of the main achievements of this paper is the extraction of such a clean and simple statement of localization for Bernoulli and other singular single site probability distributions.

We give a detailed account of this single energy multiscale analysis, which uses 'free sites' and the quantitative unique continuation principle as in [BoK] to obtain control of finite volume resonances. We also explain in detail how all forms of localization can be extracted from this single energy multiscale analysis. To put this extraction in perspective, Fröhlich and Spencer, in their seminal paper [FrS], obtained a single energy multiscale analysis for the discrete Anderson model with good probability estimates, but were not able to derive Anderson localization from their result. The desired localization was later obtained from a multiscale analysis by two different methods. Spectral averaging gets Anderson localization from a single energy multiscale analysis as in [FrS], but requires absolutely continuous single site probability distributions with a bounded density [DelLS, $\mathrm{SiW}, \mathrm{CoH} 1]$. Anderson localization, and later dynamical localization, can be proven from an energy interval multiscale analysis using generalized eigenfunctions [FrMSS, DrK1, DS, GK1, K12]. None of these methods were available in Bourgain and Kenig's setting. Spectral averaging is not feasible for Bernoulli random variables, and the energy interval multiscale analysis requires better probability estimates than are possible using the quantitative unique continuation principle. In response, Bourgain and Kenig developed a new method for obtaining Anderson localization from a single energy multiscale analysis, using Peierl's argument, generalized eigenfunctions, and two energy reductions [BouK, Section 7]. (Their method is simpler in the setting of [FrS], where the second energy reduction is not needed-see Remarks 6.13 and 6.14.) In this paper we combine the ideas of [BouK, Section 7] with methods we developed in [GK1, GK6] to extract all forms of localization from a single energy multiscale analysis, giving a detailed account of all steps.

We also derive log-Hölder continuity of the integrated density of states from the conclusions of the multiscale analysis. The multiscale analysis requires the probabilistic control of finite volume resonances subexponentially close to the given energy (and no more, as noted in [DrK1]). In [BoK] and in this article, this control is obtained as part of the multiscale analysis. We show that, in the presence of a multiscale analysis, log-Hölder continuity of the integrated density of states is the infinite volume trace of this probabilistic control (the 'Wegner estimate'). 
The integrated density of states of the discrete Anderson model is always log-Hölder continuous [CrS]. If the single site probability distribution is continuous (i.e., it has no atoms), then the integrated density of states for both discrete Anderson models and continuous Anderson Hamiltonians has at least as much regularity as the concentration function of this probability distribution [CoHK2]. Although for the discrete Anderson model there is an easy proof of continuity of the integrated density of states for arbitrary single site probability distribution [DelS], for the continuous Anderson Hamiltonian it is not even known if the integrated density of states is always a continuous function if this probability distribution has an atom.

Neither Anderson localization nor dynamical localization carry information about the regularity of the integrated density of states. Roughly speaking, dynamical localization and regularity of the integrated density of states carry complementary types of information. This is made more precise in [GK5], where we showed that for Anderson Hamiltonians with an a priori Wegner estimate, dynamical localization is necessary and sufficient to perform a multiscale analysis. The multiscale analysis contains more information than just localization properties: it also encodes regularity of the integrated density of states. This fact has been overlooked, since, prior to the multiscale analysis in [BoK], all multiscale analyses for Anderson models were performed with an a priori Wegner estimate which readily implied regularity of the integrated density of states, even without localization. In view of our results in [GK5], we may argue that, by proving both localization and log-Hölder continuity of the integrated density of states, we have extracted from the multiscale analysis all the encoded information. This 'philosophical' remark would become a mathematical statement if we could prove that localization combined with the log-Hölder continuity of the integrated density of states is enough to start a multiscale analysis, extending the results of [GK5] to the setting of this article.

The strong localization results, including Anderson localization and dynamical localization, and the log-Hölder continuity of the integrated density of states, presented in this paper for Anderson Hamiltonians, are also valid for Poisson Hamiltonians using the probabilistic properties of Poisson point processes to control the randomness of the configurations as in [GHK2].

It remains a challenge to prove localization for other random Schrödinger operators with no assumptions on the single site probability distribution except for compact support (e.g., for a Bernoulli distribution). In particular, there is no proof of localization for the multidimensional discrete Bernoulli-Anderson model, for which everything in [BoK] and this paper is valid except for the quantitative unique continuation principle; there is no unique continuation principle for discrete Schrödinger operators, where non-zero eigenfunctions may vanish on arbitrarily large sets [J, Theorem 2]. The same applies to random Landau Hamiltonians [CoH2, W1, GKS1, GKS2, GKM], where, although the unique continuation principle holds, an appropriate quantitative unique continuation principle is missing. (There is a quantitative unique continuation principle for Landau Hamiltonians, but it comes with the exponent 2 instead of $4 / 3$ [Da]. The multiscale analysis requires an exponent $<(1+\sqrt{3}) / 2$, as discused in Remark 4.8. Note that $4 / 3<(1+\sqrt{3}) / 2<2$.) The same is also true for a continuous alloy-type random Schrödinger operators with single site potentials of indefinite sign [Klo2, KloN, HK], where, although we have the 
quantitative unique continuation principle, it cannot be used to control the finite volume resonances.

This article is organized as follows:

1. Main results: In Section 1 we define Anderson Hamiltonians and state our main results, Theorem 1.2 and Corollary 1.4.

2. Anderson Hamiltonians: In Section 2 we introduce (normalized) generalized Anderson Hamiltonians, finite volume operators, and prove some basic deterministic properties. We always work with generalized Anderson Hamiltonians in the following sections.

3. Preamble to the multiscale analysis: In Section 3 we introduce the machinery for the multiscale analysis. We define 'good boxes', 'free sites', 'suitable coverings' of boxes and annuli, and prove some basic lemmas.

4. The multiscale analysis with a Wegner estimate: Section 4 is devoted to the multiscale analysis; Theorem 4.1 states the full result at the bottom of the spectrum. Proposition 4.3 gives a priori finite volume estimates at the bottom of the spectrum that yield the starting condition for the multiscale analysis. The single energy multiscale analysis with a Wegner estimate is performed in Proposition 4.6 on any energy interval where we have a priori finite volume estimates.

5. Preamble to localization: In Section 5 we introduce tools for extracting localization from the multiscale analysis. We discuss generalized eigenfunctions and the generalized eigenfunction expansion, and show that generalized eigenfunctions are small in good boxes (e.g., Lemma 5.3).

6. From the multiscale analysis to localization: In Section 6 we extract localization from the multiscale analysis. We assume that the conclusions of the multiscale analysis (i.e., of Proposition 4.6) hold for all energies in a bounded open interval (not necessarily at the bottom of the spectrum), and derive localization in that interval. Theorem 6.1 encapsulates all forms of localization.

7. Localization: In Section 7 we extract the usual forms of localization from Theorem 6.1. Anderson localization and finite multiplicity of eigenvalues are proven in Theorem 7.1. Eigenfunctions correlations (e.g., SUDEC, SULE) are obtained with probability one in Theorem 7.2 and in expectation in Theorem 7.4. Dynamical localization and decay of Fermi projections are proved with probability one in Corollary 7.3 and in expectation in Corollary 7.7 .

8. Log-Hölder continuity of the integrated density of states: In Section 8 we derive $\log$-Hölder continuity of the integrated density of states from the multiscale analysis with a Wegner estimate; see Theorem 8.1.

A. A quantitative unique continuation principle for Schrödinger operators: In Appendix A we rewrite Bourgain and Kenig's quantitative unique continuation principle for Schrödinger operators, i.e., [BoK, Lemma 3.10], in a form convenient for our purposes; see Theorem A.1 and Corollary A.2. We also give an application of this quantitative unique continuation principle to periodic Schrödinger operators, providing an alternative proof to Combes, Hislop and Klopp's lower bound estimate concerning periodic potentials and spectral projections [CoHK1, Theorem 4.1]. 


\section{Main results}

We start by defining Anderson Hamiltonians.

Definition 1.1. An Anderson Hamiltonian is a random Schrödinger operator on $\mathrm{L}^{2}\left(\mathbb{R}^{d}\right)$ of the form

$$
H_{\omega}:=-\Delta+V_{\mathrm{per}}+V_{\omega}
$$

where

(i) $\Delta$ is the $d$-dimensional Laplacian operator,

(ii) $V_{\text {per }}$ is a bounded periodic potential with period $q \in \mathbb{N}$,

(iii) $V_{\omega}$ is an alloy-type random potential,

$$
V_{\omega}(x):=\sum_{\zeta \in \mathbb{Z}^{d}} \omega_{\zeta} u(x-\zeta),
$$

where

(a) the single site potential $u$ is a non-negative bounded measurable function on $\mathbb{R}^{d}$ with compact support, uniformly bounded away from zero in a neighborhood of the origin,

(b) $\boldsymbol{\omega}=\left\{\omega_{\zeta}\right\}_{\zeta \in \mathbb{Z}^{d}}$ is a family of independent identically distributed random variables whose common probability distribution $\mu$ is non-degenerate with bounded support.

Given an Anderson Hamiltonian $H_{\omega}$, we set $P_{\omega}(B):=\chi_{B}\left(H_{\omega}\right)$ for a Borel set $B \subset \mathbb{R}^{d}, P_{\omega}(E):=P_{\omega}(\{E\})$ and $\left.\left.P_{\omega}^{(E)}:=P_{\omega}(]-\infty, E\right]\right)$ for $E \in \mathbb{R}$.

An Anderson Hamiltonian $H_{\omega}$ is a $q \mathbb{Z}^{d}$-ergodic family of random self-adjoint operators $\left(q=1\right.$ if $\left.V_{\text {per }}=0\right)$. It follows (see [KiM1, CL, PF]) that there exist fixed subsets $\Sigma$, $\Sigma_{\mathrm{pp}}, \Sigma_{\mathrm{ac}}$ and $\Sigma_{\mathrm{sc}}$ of $\mathbb{R}$ such that the spectrum $\sigma\left(H_{\omega}\right)$ of $H_{\omega}$, as well as its pure point, absolutely continuous, and singular continuous components, are equal to these fixed sets with probability one. We let $E_{\text {inf }}=\inf \Sigma>-\infty$, the bottom of the non-random spectrum; note that there exists $E_{1}>E_{\text {inf }}$ such that $\left[E_{\text {inf }}, E_{1}\right] \subset \Sigma[\mathrm{KiM} 2]$.

We will use the following notation:

- Given $x=\left(x_{1}, \ldots, x_{d}\right) \in \mathbb{R}^{d}$, we set

$$
\|x\|:=\max \left\{\left|x_{1}\right|, \ldots,\left|x_{d}\right|\right\} \quad \text { and } \quad\langle x\rangle:=\left(1+\|x\|^{2}\right)^{1 / 2} .
$$

- Given $v>0$ and $y \in \mathbb{R}^{d}$, we let $T_{v, y}$ be the operator on $\mathrm{L}^{2}\left(\mathbb{R}^{d}\right)$ given by multiplication by the function $T_{v, y}(x):=\langle x-y\rangle^{\nu}$. We set $\langle X-y\rangle:=T_{1, y}$ and $T_{v}:=T_{v, 0}=\langle X\rangle^{\nu}$.

- We let

$$
\left.\Lambda_{L}(x):=\left\{y \in \mathbb{R}^{d} ;\|y-x\|<L / 2\right\}=x+\right]-L / 2, L / 2\left[^{d}\right.
$$

denote the (open) box of side $L$ centered at $x \in \mathbb{R}^{d}$. By a box $\Lambda_{L}$ we will mean a box $\Lambda_{L}(x)$ for some $x \in \mathbb{R}^{d}$. We write $\bar{\Lambda}_{L}=\bar{\Lambda}_{L}$ for the closed box. Given scales $L_{1}<L_{2}$, we consider the (open) annulus

$$
\Lambda_{L_{2}, L_{1}}(x):=\Lambda_{L_{2}}(x) \backslash \bar{\Lambda}_{L_{1}}(x)=\left\{y \in \mathbb{R}^{d} ; L_{1} / 2<\|y-x\|<L_{2} / 2\right\},
$$

and let $\bar{\Lambda}_{L_{2}, L_{1}}(x):=\overline{\Lambda_{L_{2}, L_{1}}(x)}$ be the closed annulus. 
- Given a set $B$, we write $\chi_{B}$ for its characteristic function.

- $\chi_{x}$ will denote the characteristic function of the unit box centered at $x \in \mathbb{R}^{d}$, i.e., $\chi_{x}:=\chi_{\Lambda_{1}(x)}$.

- The cardinality of a set $A$ will be denoted by \#A.

- Given a Borel set $\Xi \subset \mathbb{R}^{d}$, we will denote its Lebesgue measure by $|\Xi|$.

- We will use the notation $\sqcup$ for disjoint unions: given sets $A$ and $B$, writing $C=A \sqcup B$ means that $C=A \cup B$ and $A \cap B=\emptyset$.

- We let $\mathcal{B}_{b}$ denote the collection of bounded complex-valued Borel functions on $\mathbb{R}$, and set $\mathcal{B}_{b, 1}:=\left\{f \in \mathcal{B}_{b} ; \sup _{t \in \mathbb{R}}|f(t)| \leq 1\right\}$.

- Given an open set $\Xi \subset \mathbb{R}^{d}$ and $n \in \mathbb{N} \cup\{\infty\}, C^{n}(\Xi)$ will denote the collection of $n$-times continuously differentiable complex-valued functions on $\Xi$, with $C_{c}^{n}(\Xi)$ denoting the subset of functions with compact support.

- By a constant we will always mean a finite constant. We will use $C_{a, b, \ldots}, C_{a, b, \ldots}^{\prime}$, $C(a, b, \ldots)$, etc., to denote a constant depending only on the parameters $a, b, \ldots$

We prove a probabilistic statement about the generalized eigenfunctions of an Anderson Hamiltonian, from which we will derive all the usual statements about localization. Generalized eigenfunctions, originally used by Martinelli and Scoppola [MS1] to extract absence of absolutely continuous from the multiscale analysis, have been an indispensable tool in all proofs of localization that do not use spectral averaging [FrMSS, DrK1, GK1, K12, BoK].

Let $H_{\omega}$ be an Anderson Hamiltonian on $\mathrm{L}^{2}\left(\mathbb{R}^{d}\right)$ and fix $v>0$. A generalized eigenfunction for a realization $H_{\omega}$ (i.e., we fix the values of the random variables $\omega$ ) with generalized eigenvalue $E \in \mathbb{R}$ is a measurable function $\psi$ on $\mathbb{R}^{d}$, with $0<\left\|T_{v}^{-1} \psi\right\|<\infty$, satisfying the eigenvalue equation for $E$ in the weak sense, i.e.,

$$
\left\langle H_{\omega} \varphi, \psi\right\rangle=E\langle\varphi, \psi\rangle \quad \text { for all } \varphi \in C_{c}^{\infty}\left(\mathbb{R}^{d}\right) .
$$

We will denote by $\Theta_{\omega}^{(v)}(E)$ the collection of generalized eigenfunctions for $H_{\omega}$ with generalized eigenvalue $E$.

To detect localization for a realization $H_{\omega}$, we introduce quantities that measure the concentration of the generalized eigenfunctions with generalized eigenvalue $E$ in certain subsets of $\mathbb{R}^{d}$. Given $x \in \mathbb{R}^{d}$, we will measure this concentration at $x$ by

$$
W_{\omega, x}^{(v)}(E):= \begin{cases}\sup _{\psi \in \Theta_{\omega}^{(v)}(E)} \frac{\left\|\chi_{x} \psi\right\|}{\left\|T_{\nu, x}^{-1} \psi\right\|} & \text { if } \Theta_{\omega}^{(v)}(E) \neq \emptyset, \\ 0 & \text { otherwise, }\end{cases}
$$

and at an annulus around $x$ at scale $L \geq 1$ by

$$
W_{\omega, x, L}^{(v)}(E):= \begin{cases}\sup _{\psi \in \Theta_{\omega}^{(\nu)}(E)} \frac{\left\|\chi_{x, L} \psi\right\|}{\left\|T_{\nu, x}^{-1} \psi\right\|} & \text { if } \Theta_{\omega}^{(v)}(E) \neq \emptyset, \\ 0 & \text { otherwise, }\end{cases}
$$

where $\chi_{x, L}:=\chi_{\Lambda_{2 L+1, L-1}(x)}$. (For technical reasons we will need an annulus slightly bigger than $\chi_{\Lambda_{2 L, L}(x)}$.) We always have $0 \leq W_{\omega, x}^{(v)}(E) \leq(5 / 4)^{v / 2}<2^{v / 2}$ and $0 \leq$ 
$W_{\omega, x, L}^{(v)}(E) \leq 2^{v / 2} L^{v}$. We will work with a fixed $v>d / 2$, but note that $W_{\omega, x}^{(v)}(E)$ and $W_{\omega, x, L}^{(v)}(E)$ are increasing in $v$.

We also prove log-Hölder continuity of the integrated density of states. The integrated density of states $N(E)$ for an Anderson Hamiltonian $H_{\omega}$, usually defined through the infinite volume limit of the normalized eigenvalue counting functions of appropriate restrictions to finite volumes (e.g., [CL, PF]), equals (e.g., [DoIM])

$$
N(E)=\frac{1}{q^{d}} \mathbb{E}\left\{\operatorname{tr}\left(\chi_{\Lambda_{q}(0)} P_{\omega}^{(E)} \chi_{\Lambda_{q}(0)}\right)\right\} \quad \text { for } E \in \mathbb{R} .
$$

The following theorem contains our main results; item (B) encapsulates localization for Anderson Hamiltonians.

Theorem 1.2. Let $H_{\omega}$ be an Anderson Hamiltonian on $\mathrm{L}^{2}\left(\mathbb{R}^{d}\right)$. For each $\left.p \in\right] 1 / 3,3 / 8[$ there exists an energy $E_{0}>E_{\mathrm{inf}}$ such that the following holds for all $\left.\tilde{p} \in\right] 0, p[$ :

(A) The integrated density of states $N(E)$ is locally log-Hölder continuous of order $\tilde{p} d$ in the interval $\left[E_{\mathrm{inf}}, E_{0}[\right.$, i.e., for all $\tilde{p} \in] 0, p\left[\right.$ and compact intervals $I \subset\left[E_{\mathrm{inf}}, E_{0}[\right.$ with length $|I| \leq 1 / 2$ we have

$$
\left|N\left(E_{2}\right)-N\left(E_{1}\right)\right| \leq \frac{C_{\widetilde{p}, I}}{|\log | E_{2}-E_{1}||^{\tilde{p} d}} \quad \text { for all } E_{1}, E_{2} \in I .
$$

(B) Let $\vartheta=\frac{1}{2} \rho^{n_{1}}$ for some $\left.\rho \in\right] 1 /(1+p), 1\left[\right.$ and $n_{1} \in \mathbb{N}$ with $\left(n_{1}+1\right) \rho^{n_{1}}<p-\tilde{p}$. There exists a constant $M>0$ such that, for every fixed $v>d / 2$, there is a finite scale $L_{0}$ such that for all $L \geq L_{0}$ and $x_{0} \in \mathbb{R}^{d}$ there exists an event $\mathcal{U}_{L, x_{0}}$ with the following properties:

(i) $\mathcal{U}_{L, x_{0}}$ depends only on the random variables $\left\{\omega_{\zeta}\right\}_{\zeta \in \Lambda_{\frac{1001 L}{500}}\left(x_{0}\right)}$, and

$$
\mathbb{P}\left\{\mathcal{U}_{L, x_{0}}\right\} \geq 1-L^{-\tilde{p} d}
$$

(ii) If $\omega \in \mathcal{U}_{L, x_{0}}$, for all $E \in\left[E_{\mathrm{inf}}, E_{0}\right.$ [ we have

$$
\text { either } W_{\boldsymbol{\omega}, x_{0}}^{(v)}(E) \leq \mathrm{e}^{-M L^{\vartheta}} \quad \text { or } \quad W_{\omega, x_{0}, L}^{(v)}(E) \leq \mathrm{e}^{-M L} .
$$

In particular, for all $\omega \in \mathcal{U}_{L, x_{0}}$ we have

$$
W_{\boldsymbol{\omega}, x_{0}}^{(v)}(E) W_{\omega, x_{0}, L}^{(v)}(E) \leq \mathrm{e}^{-\frac{1}{2} M L^{\vartheta}} \quad \text { for } E \in\left[E_{\mathrm{inf}}, E_{0}[.\right.
$$

Remark 1.3. The conclusions of Theorem 1.2 hold on any bounded open interval $\mathcal{I}$ in which we verify the starting condition (i.e., hypotheses) for the multiscale analysis of Proposition 4.6. Theorem 1.2 is stated for an interval at the bottom of the spectrum, where the starting condition for the multiscale analysis is derived from Lifshitz tail estimates in Proposition 4.3. This starting condition, and hence the analogue of Theorem 1.2, can also be proved in intervals at the edge of spectral gaps, similarly to Proposition 4.3, using the internal Lifshitz tails estimates given in [Klo3]. This starting condition is also derived in 
Proposition 4.5 for a fixed interval at the bottom of the spectrum at high disorder, provided $\mu(\{\inf \operatorname{supp} \mu\})=0$, and the conclusions of Theorem 1.2 hold in this fixed interval at high disorder if $\mu([\inf \operatorname{supp} \mu, \inf \operatorname{supp} \mu+t]) \leq C t^{\gamma}$, with $\gamma>0$ appropriately large. Note that Theorem 1.2 also holds if the single site potential $u$ in Definition 1.1 is assumed to be non-positive instead of non-negative, since in this case replacing $u$ by $-u$ and $\mu$ by $\tilde{\mu}$, where $\tilde{\mu}(B)=\mu(-B)$, rewrites the random Schrödinger operator as an Anderson Hamiltonian as in Definition 1.1.

Theorem 1.2(A) says that in the interval [ $E_{\text {inf }}, E_{0}$ [ (more generally, in the interval where we have a multiscale analysis) the integrated density of states $N(E)$ is log-Hölder continuous regardless of the (lack of) regularity of $\mu$. If the single site probability distribution $\mu$ is continuous (i.e., $\mu$ has no atoms), then it is known that the integrated density of states has at least as much regularity as the concentration function $S_{\mu}$ of $\mu$ [CoHK2]: for all compact intervals $I \subset \mathbb{R}$ we have

$$
\left|N\left(E_{2}\right)-N\left(E_{1}\right)\right| \leq C_{I} S_{\mu}\left(\left|E_{2}-E_{1}\right|\right) \quad \text { for all } E_{1}, E_{2} \in I,
$$

where $S_{\mu}(s):=\sup _{t \in \mathbb{R}} \mu([t, t+s])$ for $s \geq 0$. If $\mu$ has an atom, (1.14) is still true but useless, since $\inf _{s>0} S_{\mu}(s)>0$. For the continuous Anderson Hamiltonian it is not even known if $N(E)$ is a continuous function on $\mathbb{R}$ if $\mu$ has an atom.

Theorem 1.2(B) is a probabilistic statement about the infinite volume Anderson Hamiltonian; there is no mention of finite volume operators. It captures all the usual forms of localization. Anderson localization with finite multiplicity of eigenvalues will follow from (1.11) and (1.12) by a simple application of the Borel-Cantelli Lemma. Dynamical localization, decay of eigenfunctions correlations (e.g., SULE, SUDEC), and decay of the Fermi projections will be consequences of (1.11) and (1.13). These and other familiar localization properties are stated in Corollary 1.4. (Theorem 1.2(A) is not needed for Corollary 1.4.)

Corollary 1.4. Let $H_{\omega}$ be an Anderson Hamiltonian on $\mathrm{L}^{2}\left(\mathbb{R}^{d}\right)$. Fix $\left.p \in\right] 1 / 3,3 / 8[$, and let $\left.E_{0}>E_{\text {inf }}, \tilde{p} \in\right] 0, p\left[, \vartheta>0\right.$ and $M>0$ be as in Theorem 1.2. Then $H_{\omega}$ exhibits strong localization in the energy interval $\left[E_{\mathrm{inf}}, E_{0}\right.$ [ in the following sense:

(i) The following holds with probability one:

(a) $H_{\omega}$ has pure point spectrum in the interval $\left[E_{\mathrm{inf}}, E_{0}[\right.$.

(b) For all $E \in\left[E_{\mathrm{inf}}, E_{0}\left[, \psi \in \operatorname{Ran} P_{\omega}(E)\right.\right.$, and $v>d / 2$, we have

$$
\left\|\chi_{x} \psi\right\| \leq C_{\omega, E, v}\left\|T_{v}^{-1} \psi\right\| e^{-M\|x\|} \quad \text { for all } x \in \mathbb{R}^{d} .
$$

In particular, each eigenfunction $\psi$ of $H_{\omega}$ with eigenvalue $E \in\left[E_{\mathrm{inf}}, E_{0}[\right.$ is exponentially localized with the non-random rate of decay $M>0$.

(c) The eigenvalues of $H_{\omega}$ in $\left[E_{\mathrm{inf}}, E_{0}\right.$ [ have finite multiplicity:

$$
\operatorname{tr} P_{\omega}(E)<\infty \quad \text { for all } E \in\left[E_{\mathrm{inf}}, E_{0}[\text {. }\right.
$$

(ii) The following holds with probability one for all $\varepsilon>0$ on all compact intervals $I \subset\left[E_{\mathrm{inf}}, E_{0}[\right.$ : 
(a) For all $E \in I, x, y \in \mathbb{R}^{d}$, and $v>d / 2$, we have

$$
\left\|\chi_{x} \phi\right\|\left\|\chi_{y} \psi\right\| \leq C_{\omega, I, v, \varepsilon}\left\|T_{v}^{-1} \phi\right\|\left\|T_{\nu}^{-1} \psi\right\| \mathrm{e}^{\|x\|^{(1+\varepsilon) \vartheta / \widetilde{p}}} \mathrm{e}^{-\frac{1}{4} M\|x-y\|^{\vartheta}}
$$

for all $\phi, \psi \in \operatorname{Ran} P_{\omega}(E)$, and

$$
\left\|\chi_{x} P_{\omega}(E)\right\|_{2}\left\|\chi_{y} P_{\omega}(E)\right\|_{2} \leq C_{\omega, I, v, \varepsilon}\left\|T_{v}^{-1} P_{\omega}(E)\right\|_{2}^{2} \mathrm{e}^{\|x\|^{(1+\varepsilon) \vartheta / \widetilde{p}}} \mathrm{e}^{-\frac{1}{4} M\|x-y\|^{\vartheta}} .
$$

(b) For all $E \in I$, there exists a "center of localization" $y_{\omega, E} \in \mathbb{R}^{d}$ for all eigenfunctions with eigenvalue $E$, in the sense that for all $x \in \mathbb{R}^{d}$ and $v>d / 2$ we have

$$
\left\|\chi_{x} \phi\right\| \leq C_{\omega, I, v, \varepsilon}\left\|T_{v}^{-1} \phi\right\| \mathrm{e}^{\left\|y_{\omega, E}\right\|^{(1+\varepsilon) \vartheta / \tilde{p}}} \mathrm{e}^{-\frac{1}{4} M\left\|x-y_{\omega, E}\right\|^{\vartheta}}
$$

for all $\phi \in \operatorname{Ran} P_{\omega}(E)$, and

$$
\left\|\chi_{x} P_{\omega}(E)\right\|_{2} \leq C_{\omega, I, \nu, \varepsilon}\left\|T_{\nu}^{-1} P_{\omega}(E)\right\|_{2} \mathrm{e}^{\left\|y_{\omega, E}\right\|^{(1+\varepsilon) \vartheta / \widetilde{p}}} \mathrm{e}^{-\frac{1}{4} M\left\|x-y_{\omega, E}\right\|^{\vartheta}} .
$$

Moreover,

$$
N_{\omega, I}(L):=\sum_{\substack{E \in I \\\left\|y_{\omega, E}\right\| \leq L}} \operatorname{tr} P_{\omega}(E) \leq C_{\omega, I, \varepsilon} L^{(1+\varepsilon) d / \widetilde{p}} \quad \text { for } L \geq 1 .
$$

(c) For all $x, y \in \mathbb{R}^{d}$ we have

$$
\sup _{f \in \mathcal{B}_{b, 1}}\left\|\chi_{y} f\left(H_{\omega}\right) P_{\omega}(I) \chi_{x}\right\|_{1} \leq C_{\omega, I, \varepsilon} \mathrm{e}^{\|x\|^{(1+\varepsilon) \vartheta / \tilde{p}}} \mathrm{e}^{-\frac{1}{4} M\|x-y\|^{\vartheta}} .
$$

(d) For all $E \in I$ and $x, y \in \mathbb{R}^{d}$ we have

$$
\left\|\chi_{y} P_{\omega}^{(E)} \chi_{x}\right\|_{1} \leq C_{\omega, I, \varepsilon} \mathrm{e}^{\|x\|^{(1+\varepsilon) \vartheta / \widetilde{p}}} \mathrm{e}^{-\frac{1}{4} M\|x-y\|^{\vartheta}} .
$$

(iii) Given $b>0$, for all $s \in] 0, p /(b+1 / 2)\left[, x_{0} \in \mathbb{R}^{d}\right.$, and compact intervals $I \subset$ $\left[E_{\text {inf }}, E_{0}[\right.$, we have

$$
\begin{aligned}
& \mathbb{E}\left\{\sup _{f \in \mathcal{B}_{b, 1}}\left\|\langle X\rangle^{b d} f\left(H_{\omega}\right) P_{\omega}(I) \chi_{x_{0}}\right\|_{1}^{s}\right\}<\infty, \\
& \mathbb{E}\left\{\sup _{t \in \mathbb{R}}\left\|\langle X\rangle^{b d} \mathrm{e}^{-i t H_{\omega}} P_{\omega}(I) \chi_{x_{0}}\right\|_{1}^{s}\right\}<\infty, \\
& \mathbb{E}\left\{\sup _{E \in I}\left\|\langle X\rangle^{b d} P_{\omega}^{(E)} \chi_{x_{0}}\right\|_{1}^{s}\right\}<\infty
\end{aligned}
$$

Remark 1.5. If Theorem 1.2(B) holds on a given bounded open interval $\mathcal{I}$ (instead of the interval $\left[E_{\mathrm{inf}}, E_{0}[\right.$ at the bottom of the spectrum, as discussed in Remark 1.3), then Corollary 1.4 also holds as stated in the interval $\mathcal{I}$.

Remark 1.6. Theorem 1.2 and Corollary 1.4 also hold for Poisson Hamiltonians, with minor modifications. Their proofs can be modified for Poisson Hamiltonians using the methods of [GHK2, GHK3], both for positive and attractive Poisson potentials. 
Remark 1.7. It is instructive to compare Theorem 1.2 and Corollary 1.4 to the known results for the case when the single site probability distribution $\mu$ is absolutely continuous with a bounded density (or Hölder continuous), for which slightly stronger versions of these results have been derived from an energy-interval multiscale analysis as in [FrMSS, DrK1, FK2, GK1, GK6, K12]. In this case the probability estimate (1.11) is much stronger, one gets subexponential decay $\mathrm{e}^{-L^{\xi}}$ for any $\left.\xi \in\right] 0,1$ [ for the bad probabilities [GK1], and even exponential decay when the fractional moment method applies [AENSS]. The "either or' statement in (1.12) is stronger: either $W_{\boldsymbol{\omega}, x_{0}}^{(v)}(E) \leq \mathrm{e}^{-M L}$ or $W_{\omega, x_{0}, L}^{(v)}(E) \leq \mathrm{e}^{-M L}$. We also have exponential decay in (1.13) and in Corollary 1.4(ii), that is, they hold with $\vartheta=1$. Corollary 1.4(iii) holds for all $b>0$ with $s=1$. The SUDEC estimate (1.17) and the SULE estimate (1.19) hold with exponential decay and milder than exponential growth in $x$ or $y$; moreover they are equivalent, one can be derived from the other (see [GK1, GK6]). But in the general case (1.17) and (1.19) are not equivalent; (1.17) implies (1.19) but the converse is not true.

Theorem 1.2 and Corollary 1.4 will be proved in the context of generalized Anderson Hamiltonians. Theorem 1.2(A) is proven in Theorem 8.1, and Theorem 1.2(B) is contained in Theorem 6.1. Corollary 1.4(i) is proven in Theorem 7.1, Corollary 1.4(ii) in Theorem 7.2 and Corollary 7.3, and Corollary 1.4(iii) follows from Corollary 7.7.

\section{Anderson Hamiltonians}

\subsection{Normalized Anderson Hamiltonians}

Given an Anderson Hamiltonian $H_{\omega}$, it follows from Definition 1.1 that the common probability distribution $\mu$ of the random variables $\omega=\left\{\omega_{\zeta}\right\}_{\zeta \in \mathbb{Z}^{d}}$ satisfies

$$
\left\{M_{-}, M_{+}\right\} \subset \operatorname{supp} \mu \subset\left[M_{-}, M_{+}\right] \quad \text { for some }-\infty<M_{-}<M_{+}<\infty .
$$

Letting

$$
\begin{aligned}
\widehat{V}_{\text {per }} & =\widetilde{V}_{\text {per }}-\inf \sigma\left(-\Delta+\widetilde{V}_{\text {per }}\right) \quad \text { with } \quad \widetilde{V}_{\text {per }}(x)=V_{\text {per }}(x)+M_{-} \sum_{\zeta \in \mathbb{Z}^{d}} u(x-\zeta), \\
\widehat{V}_{\widehat{\omega}}(x) & =\sum_{\zeta \in \mathbb{Z}^{d}} \widehat{\omega}_{\zeta} \widehat{u}(x-\zeta) \quad \text { with } \widehat{u}=\left(M_{+}-M_{-}\right) u \text { and } \widehat{\omega}_{\zeta}=\frac{\omega_{\zeta}-M_{-}}{M_{+}-M_{-}}, \\
\widehat{H}_{\widehat{\omega}} & =-\Delta+\widehat{V}_{\text {per }}+\widehat{V}_{\widehat{\boldsymbol{\omega}}},
\end{aligned}
$$

we have

$$
H_{\omega}=\widehat{H}_{\widehat{\omega}}+\inf \sigma\left(-\Delta+\widetilde{V}_{\text {per }}\right) .
$$

Since $\widehat{H}_{\widehat{\omega}}$ is a normalized Anderson Hamiltonian as in Definition 2.1 below, we conclude that every Anderson Hamiltonian equals a normalized Anderson Hamiltonian plus a constant. Thus, without loss of generality, it suffices to study normalized Anderson Hamiltonians as in Definition 2.1, which makes the relevant parameters explicit. 
Definition 2.1. A normalized Anderson Hamiltonian is an Anderson Hamiltonian $H_{\omega}$ such that:

(i) the periodic potential $V_{\text {per }}$ satisfies

$$
\inf \sigma\left(-\Delta+V_{\text {per }}\right)=0,
$$

(ii) the single site potential $u$ is a measurable function on $\mathbb{R}^{d}$ with

$$
\left.u_{-} \chi_{\Lambda_{\delta_{-}}(0)} \leq u \leq u_{+} \chi_{\Lambda_{\delta_{+}}(0)} \text { for some constants } u_{ \pm}, \delta_{ \pm} \in\right] 0, \infty[,
$$

(iii) $\boldsymbol{\omega}=\left\{\omega_{\zeta}\right\}_{\zeta \in \mathbb{Z}^{d}}$ is a family of independent, identically distributed random variables with a common probability distribution $\mu$ satisfying

$$
\{0,1\} \subset \operatorname{supp} \mu \subset[0,1] .
$$

The condition (2.4) implies that $\left[0, E_{1}\right] \subset \sigma\left(H_{0}\right)$ for some $E_{1}>0$. It follows that the non-random spectrum $\Sigma$ of a normalized Anderson Hamitonian $H_{\boldsymbol{\omega}}$ satisfies (see [KiM2])

$$
\sigma\left(H_{0}\right) \subset \Sigma \subset[0, \infty[
$$

so

$$
\inf \Sigma=0 \quad \text { and } \quad\left[0, E_{1}\right] \subset \Sigma \text { for some } E_{1}=E_{1}\left(V_{\text {per }}\right)>0 .
$$

In particular, we have

$$
\Sigma=\sigma(-\Delta)=\left[0, \infty\left[\text { if } \quad V_{\text {per }}=0\right.\right.
$$

\subsection{Generalized Anderson Hamiltonians}

We will conduct our analysis of normalized Anderson Hamiltonians in a more general context which incorporates an additional background potential, bounded and nonnegative, but otherwise arbitrary, and allows variability in the single site potentials as long as they satisfy uniform bounds.

Definition 2.2. A generalized (normalized) Anderson Hamiltonian is a random Schrödinger operator on $\mathrm{L}^{2}\left(\mathbb{R}^{d}\right)$ of the form

$$
H_{\omega}=H_{0}+V_{\omega} \quad \text { with } \quad H_{0}=-\Delta+V_{\text {per }}+U,
$$

where $V_{\text {per }}$ is a bounded periodic potential with period $q \in \mathbb{N}$ such that

$$
\inf \sigma\left(-\Delta+V_{\text {per }}\right)=0,
$$

$U$ is a measurable function on $\mathbb{R}^{d}$ satisfying

$$
0 \leq U(x) \leq U_{+} \quad \text { for all } x \in \mathbb{R}^{d} \text { for some constant } U_{+} \in[0, \infty[,
$$

and $V_{\omega}$ is the random potential

$$
V_{\omega}(x):=\sum_{\zeta \in \mathbb{Z}^{d}} \omega_{\zeta} u_{\zeta}(x),
$$


where the family $\boldsymbol{\omega}=\left\{\omega_{\zeta}\right\}_{\zeta \in \mathbb{Z}^{d}}$ of random variables is as in Definition 2.1, and $\mathbf{u}=$ $\left\{u_{\zeta}\right\}_{\zeta \in \mathbb{Z}^{d}}$ is a family of measurable functions on $\mathbb{R}^{d}$ such that there are constants $u_{ \pm}, \delta_{ \pm} \in$ ] $0, \infty[$ for which

$$
u_{-} \chi_{\Lambda_{\delta_{-}}(\zeta)} \leq u_{\zeta} \leq u_{+} \chi_{\Lambda_{\delta_{+}}(\zeta)} \quad \text { for all } \zeta \in \mathbb{Z}^{d}
$$

Without loss of generality, we realize the random variables $\left\{\omega_{\zeta}\right\}_{\zeta \in \mathbb{Z}^{d}}$ as the coordinate functions on the probability space $(\Omega, \mathcal{F}, \mathbb{P})$, where $\Omega=[0,1]^{\mathbb{Z}^{d}}, \mathcal{F}$ denotes the $\sigma$ algebra generated by the coordinate functions, and $\mathbb{P}=\mu^{\mathbb{Z}^{d}}$, the product measure of $\mathbb{Z}^{d}$ copies of the common probability distribution $\mu$ of the random variables $\left\{\omega_{\zeta}\right\}_{\zeta \in \mathbb{Z}^{d}}$. In other words, $(\Omega, \mathcal{F}, \mathbb{P})=\left([0,1], \mathcal{B}_{[0,1]}, \mu\right)^{\mathbb{Z}^{d}}$, the product measure space of $\mathbb{Z}^{d}$ copies of the measure space $\left([0,1], \mathcal{B}_{[0,1]}, \mu\right)$, where $\mathcal{B}_{[0,1]}$ is the Borel $\sigma$-algebra on $[0,1]$. The expectation with respect to $\mathbb{P}$ will be denoted by $\mathbb{E}$. Note that $\Omega$ is a compact Hausdorff space with the product topology and $\mathcal{F}$ is the corresponding Borel $\sigma$-algebra. A set $\mathcal{U} \in \mathcal{F}$ will be called an event.

A generalized Anderson Hamiltonian $H_{\omega}$ is a measurable map from the probability space $(\Omega, \mathcal{F}, \mathbb{P})$ to self-adjoint operators on the Hilbert space $\mathrm{L}^{2}\left(\mathbb{R}^{d}\right)$. Measurability of $H_{\omega}$ means that the maps $\omega \mapsto f\left(H_{\omega}\right)$ are weakly (and hence strongly) measurable for all bounded Borel measurable functions $f$ on $\mathbb{R}$.

A generalized Anderson Hamiltonian $H_{\omega}$ is not, in general, a $q \mathbb{Z}^{d}$-ergodic family of random self-adjoint operators for any $q \in \mathbb{N}$, so the spectrum of $H_{\boldsymbol{\omega}}$, as well as its pure point, absolutely continuous, and singular continuous components, need not be nonrandom (i.e., equal to some fixed set with probability one). But we always have $\sigma\left(H_{\omega}\right) \subset$ $[0, \infty)$ for all $\omega \in \Omega$.

\subsection{Finite volume Anderson Hamiltonians}

Given a set $\Xi \subset \mathbb{R}^{d}$, we set $\widetilde{\Xi}:=\Xi \cap \mathbb{Z}^{d}$ and consider the product measure space $\left(\Omega_{\Xi}, \mathcal{F}_{\Xi}, \mathbb{P}_{\Xi}\right)=\left([0,1], \mathcal{B}_{[0,1]}, \mu\right)^{\widetilde{\Xi}}$; in particular, $\Omega_{\Xi}=[0,1]^{\widetilde{\Xi}}$. We identify $\mathcal{F}_{\Xi}$ with the sub- $\sigma$-algebra of subsets of $\Omega$ generated by the coordinate functions $\omega_{\Xi}=\left\{\omega_{\zeta}\right\}_{\zeta \in \widetilde{\Xi}}$, in which case $\mathbb{P}_{\Xi}$ is the restriction of $\mathbb{P}$ to $\mathcal{F}_{\Xi}$.

Given a generalized Anderson Hamiltonian $H_{\omega}$, we set

$$
V_{\omega_{\Xi}}(x):=\sum_{\zeta \in \widetilde{\Xi}} \omega_{\zeta} u_{\zeta}(x) \quad \text { for } \omega \in \Omega \text { and } \Xi \subset \mathbb{R}^{d},
$$

and define the corresponding finite volume (generalized) Anderson Hamiltonian on a box $\Lambda=\Lambda_{L}(x)$ in $\mathbb{R}^{d}$ as follows:

$$
H_{\omega, \Lambda}:=H_{0, \Lambda}+V_{\omega, \Lambda} \quad \text { on } \mathrm{L}^{2}(\Lambda)
$$

with

$$
H_{0, \Lambda}:=-\Delta_{\Lambda}+V_{\text {per }, \Lambda}+U_{\Lambda},
$$

where $\Delta_{\Lambda}$ is the Laplacian on $\Lambda$ with a Dirichlet boundary condition, and $V_{\mathrm{per}, \Lambda}, U_{\Lambda}$ and $V_{\omega, \Lambda}$ are the restrictions of $V_{\text {per }}, U$ and $V_{\omega_{\Lambda}}$ to $\Lambda$. Since we are using a Dirichlet boundary 
condition, we always have inf $\sigma\left(H_{0, \Lambda}\right) \geq 0$ (easy to see using quadratic forms), and hence $\inf \sigma\left(H_{\omega, \Lambda}\right) \geq 0$. The finite volume resolvent, defined for $z \notin \sigma\left(H_{\omega, \Lambda}\right)$ by

$$
R_{\omega, \Lambda}(z):=\left(H_{\omega, \Lambda}-z\right)^{-1} \quad \text { on } \mathrm{L}^{2}(\Lambda),
$$

is a compact operator. Note that $\Delta_{\Lambda}=\nabla_{\Lambda} \cdot \nabla_{\Lambda}$, where $\nabla_{\Lambda}$ is the gradient with a Dirichlet boundary condition.

We will identify $\mathrm{L}^{2}(\Lambda)$ with $\chi_{\Lambda} \mathrm{L}^{2}\left(\mathbb{R}^{d}\right)$ when convenient, and, if necessary, we will use subscripts $\Lambda$ and $\mathbb{R}^{d}$ to distinguish between the norms and inner products of $\mathrm{L}^{2}(\Lambda)$ and $\mathrm{L}^{2}\left(\mathbb{R}^{d}\right)$. In particular, we use the identification $V_{\mathrm{per}, \Lambda}=\chi_{\Lambda} V_{\mathrm{per}}, U_{\Lambda}=\chi_{\Lambda} U$, and $V_{\omega, \Lambda}=\chi_{\Lambda} V_{\omega_{\Lambda}}$. If $\Lambda \subset \Lambda^{\prime}$, we will also extend operators on $\mathrm{L}^{2}(\Lambda)$, such as $R_{\omega, \Lambda}(z)$, to operators on $\mathrm{L}^{2}\left(\Lambda^{\prime}\right)$ by making them the zero operator on $\mathrm{L}^{2}\left(\Lambda^{\prime} \backslash \Lambda\right)$. If $\eta \in \mathrm{L}^{\infty}(\Lambda)$, we will also use $\eta$ to denote the operator given by multiplication by $\eta$ on $\mathrm{L}^{2}(\Lambda)$.

If $\Xi \subset \mathbb{R}^{d}, \bar{\Xi}$ will denote its closure, $\Xi^{0}$ its interior, and $\partial \Xi:=\bar{\Xi} \backslash \Xi^{0}$ its boundary. If $\Xi \subset \Xi^{\prime} \subset \mathbb{R}^{d}, \partial^{\Xi^{\prime}} \Xi:=\partial \Xi \backslash \partial \Xi^{\prime}$ will denote the boundary of $\Xi$ in $\Xi^{\prime}$. ( $\partial^{\Xi^{\prime}} \Xi$ is the boundary of $\Xi$ with respect to the relative topology on $\Xi^{\prime}$.)

Given a box $\Lambda \subset \Lambda^{\prime}$, where $\Lambda^{\prime}$ is either a box or $\mathbb{R}^{d}$, and $\delta>0$, we set (the distance is given by the norm in (1.3))

$$
\begin{aligned}
\Lambda^{\Lambda^{\prime}, \delta} & :=\left\{x \in \Lambda ; \Lambda_{2 \delta}(x) \cap \Lambda^{\prime} \subset \Lambda\right\}=\left\{x \in \Lambda ; \operatorname{dist}\left(x, \partial^{\Lambda^{\prime}} \Lambda\right) \geq \delta\right\}, \\
\partial^{\Lambda^{\prime}, \delta} \Lambda & :=\Lambda \backslash \Lambda^{\Lambda^{\prime}, \delta} .
\end{aligned}
$$

If $\Lambda^{\prime}=\mathbb{R}^{d}$ we generally omit it from the notation.

In general $V_{\omega, \Lambda} \neq \chi_{\Lambda} V_{\omega, \Lambda^{\prime}}$ for $\Lambda \subset \Lambda^{\prime}$, but we always have

$$
\chi_{\Lambda^{\Lambda^{\prime}, \delta_{+} / 2}} V_{\omega, \Lambda}=\chi_{\Lambda^{\Lambda^{\prime}, \delta_{+} / 2}} V_{\omega, \Lambda^{\prime}}
$$

In this paper we will always assume that the finite volumes $\Lambda=\Lambda_{L}$ where we define $H_{\omega, \Lambda}$ have $L \geq 100\left(\delta_{+}+1\right)$.

\subsection{Generalized eigenfunctions}

Let $H_{\omega}$ be a generalized Anderson Hamiltonian, fix $\omega \in \Omega$, and let $\Lambda$ be either $\mathbb{R}^{d}$ or a box $\Lambda_{L}$. Recall that $\mathcal{D}\left(H_{\omega, \Lambda}\right)=\mathcal{D}\left(\Delta_{\Lambda}\right)$.

Definition 2.3. A generalized eigenfunction for $H_{\omega, \Lambda}$ with generalized eigenvalue $E \in \mathbb{R}$ is a measurable function $\psi$ on $\Lambda$ with

$$
0<\left\|T_{v}^{-1} \psi\right\|_{\Lambda}<\infty \quad \text { for some } v>0,
$$

such that

$$
\left\langle H_{\omega, \Lambda} \varphi, \psi\right\rangle=E\langle\varphi, \psi\rangle \quad \text { for all } \varphi \in C_{c}^{\infty}(\Lambda)
$$


It follows (e.g., [K1KS]) that if $\psi$ is a generalized eigenfunction for $H_{\omega, \Lambda}$ with generalized eigenvalue $E \in \mathbb{R}$, then for all $\phi \in C_{c}^{2}(\Lambda)$ we have $\phi \psi \in \mathcal{D}\left(\Delta_{\Lambda}\right) \subset \mathcal{D}\left(\nabla_{\Lambda}\right)$ and

$$
\left(H_{\omega, \Lambda}-E\right) \phi \psi=W_{\Lambda}(\phi) \psi,
$$

where $W_{\Lambda}(\phi)$ is the closed densely defined operator on $\mathrm{L}^{2}(\Lambda)$ given by

$$
W_{\Lambda}(\phi)=-2(\nabla \phi) \cdot \nabla_{\Lambda}-\Delta \phi
$$

(More precisely, $W_{\Lambda}(\phi) \psi:=W_{\Lambda}(\phi) \tilde{\phi} \psi$ for all $\widetilde{\phi} \in C_{c}^{2}(\Lambda)$ such that $\widetilde{\phi} \equiv 1$ on $\operatorname{supp} \phi$.)

Eigenfunctions are always generalized eigenfunctions.

\subsection{Properties of finite volume operators}

We will now derive some deterministic properties of the finite volume operators corresponding to a generalized Anderson Hamiltonian $H_{\omega}$.

Given $\Lambda$, either a finite box or $\mathbb{R}^{d}$, and $x, y \in \Lambda,\left\|\chi_{y} R_{\omega, \Lambda}(z) \chi_{x}\right\| \in[0, \infty[$ is well defined for $z \notin \sigma\left(H_{\omega, \Lambda}\right)$. We will abuse the notation and make the extension to $z \in \sigma\left(H_{\omega, \Lambda}\right)$ by

$$
\left\|\chi_{y} R_{\omega, \Lambda}(z) \chi_{x}\right\|:=\limsup _{\varepsilon \rightarrow 0}\left\|\chi_{y} R_{\omega, \Lambda}(z+i \varepsilon) \chi_{x}\right\| \in[0, \infty] .
$$

We will consider boxes $\Lambda \subset \Lambda^{\prime}$ without requiring the interior box $\Lambda$ to be at a certain distance from the boundary of $\Lambda^{\prime}$. For this reason we work with $\partial^{\Lambda^{\prime}} \Lambda$ (the boundary of $\Lambda$ in $\Lambda^{\prime}$ ) instead of $\partial \Lambda$.

Lemma 2.4. Consider a box $\Lambda=\Lambda_{\ell} \subset \Lambda^{\prime}$, where $\Lambda^{\prime}$ is either a finite box or $\mathbb{R}^{d}$, and let $z \notin \sigma\left(H_{\omega, \Lambda}\right)$. Then, given $x \in \Lambda$ with $\Lambda_{\delta_{+}+3}(x) \cap \Lambda^{\prime} \subset \Lambda$ and $y \in \Lambda^{\prime}$, we can find $x^{\prime} \in \Upsilon_{\Lambda}^{\Lambda^{\prime}}$, where

$$
\Upsilon_{\Lambda}^{\Lambda^{\prime}}:=\left\{x \in \Lambda ; \operatorname{dist}\left(x, \partial^{\Lambda^{\prime}} \Lambda\right)=\left(\delta_{+}+1\right) / 2\right\}
$$

such that

$$
\begin{aligned}
& \left\|\chi_{y} R_{\omega, \Lambda^{\prime}}(z) \chi_{x}\right\| \\
& \quad \leq\left\|\chi_{y} \chi_{\Lambda^{\left(\Lambda^{\prime}, 1 / 2\right)}} R_{\omega, \Lambda}(z) \chi_{x}\right\|+\gamma_{z} \ell^{d-1}\left\|\chi_{y} R_{\omega, \Lambda^{\prime}}(z) \chi_{x^{\prime}}\right\|\left\|\chi_{x^{\prime}} R_{\omega, \Lambda}(z) \chi_{x}\right\|,
\end{aligned}
$$

with

$$
\gamma_{z}=\gamma_{z, d, V_{\mathrm{per}}}=C_{d}\left(1+\max \left\{0, \Re z-\operatorname{ess} \inf V_{\mathrm{per}}\right\}\right)^{1 / 2}
$$

In particular,

(i) if $y \in \Lambda^{\prime} \backslash \Lambda$, we have

$$
\left\|\chi_{y} R_{\omega, \Lambda^{\prime}}(z) \chi_{x}\right\| \leq \gamma_{z} \ell^{d-1}\left\|\chi_{y} R_{\omega, \Lambda^{\prime}}(z) \chi_{x^{\prime}}\right\|\left\|\chi_{x^{\prime}} R_{\omega, \Lambda}(z) \chi_{x}\right\|,
$$


(ii) if $y \in \Lambda$, we have

$$
\begin{aligned}
& \left\|\chi_{y} R_{\omega, \Lambda^{\prime}}(z) \chi_{x}\right\| \\
& \quad \leq\left\|\chi_{y} R_{\omega, \Lambda}(z) \chi_{x}\right\|+\gamma_{z} \ell^{d-1}\left\|\chi_{y} R_{\omega, \Lambda^{\prime}}(z) \chi_{x^{\prime}}\right\|\left\|\chi_{x^{\prime}} R_{\omega, \Lambda}(z) \chi_{x}\right\| .
\end{aligned}
$$

Proof. Given boxes $\Lambda \subset \Lambda^{\prime}$, we let $\Upsilon_{\Lambda}^{\Lambda^{\prime}}$ be as in (2.26) and set

$$
\widehat{\Upsilon}=\widehat{\Upsilon}_{\Lambda}^{\Lambda^{\prime}}:=\left\{x \in \Lambda ; \operatorname{dist}\left(x, \Upsilon_{\Lambda}^{\Lambda^{\prime}}\right)<1 / 4\right\}=\left\{\bigcup_{y^{\prime} \in \Upsilon_{\Lambda}^{\Lambda^{\prime}}} \Lambda_{1 / 2}\left(y^{\prime}\right)\right\} \cap \Lambda .
$$

There exists a constant $C_{d}$, independent of $\Lambda$ and $\Lambda^{\prime}$, for which we can find a function $\phi=\phi_{\Lambda}^{\Lambda^{\prime}} \in C^{2}\left(\Lambda^{\prime}\right)$, with $0 \leq \phi \leq 1$, such that

$$
\begin{aligned}
& \phi \equiv 1 \quad \text { on } \Lambda^{\Lambda^{\prime},\left(\delta_{+}+1\right) / 2+1 / 4}, \\
& \phi \equiv 0 \quad \text { on } \Lambda^{\prime} \backslash \Lambda^{\Lambda^{\prime},\left(\delta_{+}+1\right) / 2-1 / 4}, \\
& |\nabla \phi|,|\Delta \phi| \leq C_{d} .
\end{aligned}
$$

Note that

$$
\operatorname{supp} \phi \subset \Lambda^{(1)}:=\Lambda^{\Lambda^{\prime},\left(\delta_{+}+1\right) / 2-(1 / 4)} \quad \text { and } \quad \operatorname{supp} \nabla \phi \subset \widehat{\Upsilon}=\widehat{\Upsilon}_{\Lambda}^{\Lambda^{\prime}} .
$$

In particular, we have $\phi \mathcal{D}\left(\Delta_{\Lambda}\right) \subset \mathcal{D}\left(\Delta_{\Lambda}\right)$ and $\phi \mathcal{D}\left(\Delta_{\Lambda^{\prime}}\right) \subset \mathcal{D}\left(\Delta_{\Lambda^{\prime}}\right)$.

Suppose first that $z \notin \sigma\left(H_{\omega, \Lambda}\right) \cup \sigma\left(H_{\omega, \Lambda^{\prime}}\right)$. In this case we use the geometric resolvent identity (cf. [CoH1, FK2, BoK]). In view of (2.20), if $z \notin \sigma\left(H_{\omega, \Lambda}\right) \cup \sigma\left(H_{\omega, \Lambda^{\prime}}\right)$ we get

$$
R_{\omega, \Lambda^{\prime}}(z) \phi=\phi R_{\omega, \Lambda}(z)+R_{\omega, \Lambda^{\prime}}(z) W_{\Lambda}(\phi) R_{\omega, \Lambda}(z),
$$

as operators from $\mathrm{L}^{2}(\Lambda)$ to $\mathrm{L}^{2}\left(\Lambda^{\prime}\right)$, where $W_{\Lambda}(\phi)$ is as in (2.24). Given $x \in \Lambda$ with $\Lambda_{\delta_{+}+3}(x) \cap \Lambda^{\prime} \subset \Lambda$, i.e., $x \in \Lambda^{\Lambda^{\prime},\left(\delta_{+}+1\right) / 2+1}$, we have

$$
\chi_{x}=\phi \chi_{x}, \quad \chi_{y^{\prime}} \chi_{x}=0 \quad \text { for } y^{\prime} \in \Upsilon_{\Lambda}^{\Lambda^{\prime}} .
$$

It follows that for $y \in \Lambda^{\prime}$ we have

$$
\begin{aligned}
\chi_{y} R_{\omega, \Lambda^{\prime}}(z) \chi_{x} & =\chi_{y} R_{\omega, \Lambda^{\prime}}(z) \phi \chi_{x} \\
& =\chi_{y} \phi R_{\omega, \Lambda}(z) \chi_{x}+\chi_{y} R_{\omega, \Lambda^{\prime}}(z) W_{\Lambda}(\phi) R_{\omega, \Lambda}(z) \chi_{x} \\
& =\chi_{y} \phi R_{\omega, \Lambda}(z) \chi_{x}+\chi_{y} R_{\omega, \Lambda^{\prime}}(z) \chi_{\widehat{\Upsilon}} W_{\Lambda}(\phi) R_{\omega, \Lambda}(z) \chi_{x} .
\end{aligned}
$$

Let $\ell$ be the length of the side of the box $\Lambda$, i.e., $\Lambda=\Lambda_{\ell}$. Then we can pick $y_{1}, \ldots, y_{J} \in \Upsilon_{\Lambda}^{\Lambda^{\prime}}$, where $C_{d}^{\prime} \ell^{d-1} \leq J \leq C_{d}^{\prime \prime} \ell^{d-1}$, and $y_{1}^{\prime}, \ldots, y_{J^{\prime}}^{\prime} \in \overline{\Upsilon_{\Lambda}^{\Lambda^{\prime}}} \backslash \Upsilon_{\Lambda}^{\Lambda^{\prime}}$, with $0 \leq J^{\prime} \leq C_{d}^{\prime \prime \prime} \ell^{d-2}$ (note $J^{\prime}=0$ if $\partial^{\Lambda^{\prime}} \Lambda=\partial \Lambda$, in which case $\overline{\Upsilon_{\Lambda}^{\Lambda^{\prime}}}=\Upsilon_{\Lambda}^{\Lambda^{\prime}}$ ), such that $\Lambda_{1}\left(y_{j}\right) \subset \Lambda$ for $j=1, \ldots, J$,

$$
\widehat{\Upsilon}_{\Lambda}^{\Lambda^{\prime}}=\left\{\bigcup_{j=1}^{J} \bar{\Lambda}_{1 / 2}\left(y_{j}\right)\right\} \cup\left\{\bigcup_{j^{\prime}=1}^{J^{\prime}} \bar{\Lambda}_{1 / 2}\left(y_{j^{\prime}}^{\prime}\right) \cap \bar{\Lambda}\right\},
$$


and $y_{1}, \ldots, y_{J}, y_{1}^{\prime}, \ldots, y_{J^{\prime}}^{\prime}$ form a minimal set with respect to these properties. It follows that we can select disjoint open sets $\mathcal{O}_{j} \subset \Lambda_{1 / 2}\left(y_{j}\right)$ and $\mathcal{O}_{j^{\prime}}^{\prime} \subset \Lambda_{1 / 2}\left(y_{j^{\prime}}^{\prime}\right) \cap \Lambda$, where $j=1, \ldots, J$ and $j^{\prime}=1, \ldots, J^{\prime}$, such that

$$
\overline{\widehat{\Upsilon}_{\Lambda}^{\Lambda^{\prime}}}=\left\{\bigcup_{j=1}^{J} \overline{\mathcal{O}_{j}}\right\} \cup\left\{\bigcup_{j^{\prime}=1}^{J^{\prime}} \overline{\mathcal{O}_{j^{\prime}}^{\prime}}\right\} .
$$

It follows that

$$
\begin{aligned}
& \left\|\chi_{y} R_{\boldsymbol{\omega}, \Lambda^{\prime}}(z) \chi_{\widehat{\Upsilon}} W_{\Lambda}(\phi) R_{\boldsymbol{\omega}, \Lambda}(z) \chi_{x}\right\| \\
& \leq \sum_{j=1}^{J}\left\|\chi_{y} R_{\boldsymbol{\omega}, \Lambda^{\prime}}(z) \chi_{\mathcal{O}_{j}} W_{\Lambda}(\phi) R_{\boldsymbol{\omega}, \Lambda}(z) \chi_{x}\right\|+\sum_{j^{\prime}=1}^{J^{\prime}}\left\|\chi_{y} R_{\boldsymbol{\omega}, \Lambda^{\prime}}(z) \chi_{\mathcal{O}_{j^{\prime}}^{\prime}} W_{\Lambda}(\phi) R_{\boldsymbol{\omega}, \Lambda}(z) \chi_{x}\right\| \\
& \leq \sum_{j=1}^{J}\left\{\left\|\chi_{y} R_{\boldsymbol{\omega}, \Lambda^{\prime}}(z) \chi_{\Lambda_{1 / 2}\left(y_{j}\right)}\right\|\left\|\chi_{\Lambda_{1 / 2}\left(y_{j}\right)} W_{\Lambda}(\phi) R_{\boldsymbol{\omega}, \Lambda}(z) \chi_{x}\right\|\right\} \\
& \quad+\sum_{j^{\prime}=1}^{J^{\prime}}\left\{\left\|\chi_{y} R_{\boldsymbol{\omega}, \Lambda^{\prime}}(z) \chi_{\Lambda_{1 / 2}\left(y_{j^{\prime}}^{\prime}\right) \cap \Lambda}\right\|\left\|\chi_{\Lambda_{1 / 2}\left(y_{j^{\prime}}^{\prime}\right) \cap \Lambda} W_{\Lambda}(\phi) R_{\boldsymbol{\omega}, \Lambda}(z) \chi_{x}\right\|\right\}
\end{aligned}
$$

Let $\Lambda_{\sharp}$ be either $\Lambda_{1 / 2}\left(y_{j}\right)=\Lambda_{1 / 2}\left(y_{j}\right) \cap \Lambda$ or $\Lambda_{1 / 2}\left(y_{j^{\prime}}^{\prime}\right) \cap \Lambda$ for some $j$ or $j^{\prime}$. We write $\Lambda_{\sharp}^{\prime}$ for the corresponding $\Lambda_{1}\left(y_{j}\right) \cap \Lambda$ or $\Lambda_{1}\left(y_{j^{\prime}}^{\prime}\right) \cap \Lambda$. Using (2.24) and (2.34) we get

$$
\left\|\chi_{\Lambda_{\sharp}} W_{\Lambda}(\phi) R_{\omega, \Lambda}(z) \chi_{x}\right\| \leq 2 C_{d}\left\|\chi_{\Lambda_{\sharp}} \nabla_{\Lambda} R_{\omega, \Lambda}(z) \chi_{x}\right\|+C_{d}\left\|\chi_{\Lambda_{\sharp}} R_{\omega, \Lambda}(z) \chi_{x}\right\| .
$$

We now use the following interior estimate (e.g., [GK5, Lemma A.2]): Let $\eta \in C^{1}(\mathcal{O})$ with $\|\eta\|_{\infty} \leq 1$, where $\mathcal{O} \subset \mathbb{R}^{d}$ is an open set. Given a finite box $\Lambda$ such that $\bar{\Lambda} \subset \mathcal{O}$, we set $\eta_{\Lambda}=\eta \chi_{\Lambda}$. Then, for all $\omega \in[0,1]^{\mathbb{Z}^{d}}, z \in \mathbb{C}$, and $\psi \in \mathcal{D}\left(\Delta_{\Lambda}\right)$, we have

$$
\begin{aligned}
\left\|\eta_{\Lambda} \nabla_{\Lambda} \psi\right\|^{2} \leq & \left\|\chi_{\operatorname{supp} \eta_{\Lambda}}\left(H_{\omega, \Lambda}-z\right) \psi\right\|^{2} \\
& +\left(1+\max \left\{0, \Re z-\operatorname{ess} \inf V_{\text {per }}\right\}+4\left\|\nabla \eta_{\Lambda}\right\|_{\infty}^{2}\right)\left\|\chi_{\operatorname{supp} \eta_{\Lambda}} \psi\right\|^{2}
\end{aligned}
$$

(Although [GK5, Lemma A.2] is stated with somewhat different conditions on $\eta$, the proof applies with $\eta$ as above. The important observation is that with the Dirichlet boundary condition we have $\eta \psi=\eta_{\Lambda} \psi \in \mathcal{D}\left(\Delta_{\Lambda}\right)$ for all $\psi \in \mathcal{D}\left(\Delta_{\Lambda}\right)$.)

Given a box $\Lambda_{1 / 2}\left(x^{\prime}\right)$, we fix a function $\eta \in C^{1}\left(\mathbb{R}^{d}\right)$ with $0 \leq \eta \leq 1$ such that $\eta \equiv 1$ on $\Lambda_{1 / 2}\left(x^{\prime}\right)$, supp $\eta \subset \Lambda_{1}\left(x^{\prime}\right)$, and $\|\nabla \eta\|_{\infty} \leq C_{d}^{\prime \prime \prime}$. We have, using (2.43) and $\eta \chi_{x}=0$ (see (2.37)),

$$
\left\|\chi_{\Lambda_{\sharp}} \nabla_{\Lambda} R_{\omega, \Lambda}(z) \chi_{x}\right\| \leq\left\|\eta_{\Lambda} \nabla_{\Lambda} R_{\omega, \Lambda}(z) \chi_{x}\right\| \leq \gamma_{\Re z, d, V_{\mathrm{per}}}^{\prime}\left\|\chi_{\Lambda_{\sharp}^{\prime}} R_{\omega, \Lambda}(z) \chi_{x}\right\|,
$$

with

$$
\gamma_{\mathfrak{R} z, d, V_{\mathrm{per}}}^{\prime}:=C_{d}^{\prime \prime \prime}\left(1+\max \left\{0, \Re z-\operatorname{ess} \inf V_{\mathrm{per}}\right\}\right)^{1 / 2}
$$


If $\Lambda_{\sharp}=\Lambda_{1 / 2}\left(y_{j}\right)$, we have $\chi_{\Lambda_{\sharp}} \leq \chi_{\Lambda_{\sharp}^{\prime}}=\chi_{y_{j}}$. If $\Lambda_{\sharp}=\Lambda_{1 / 2}\left(y_{j^{\prime}}^{\prime}\right) \cap \Lambda$, we have $\chi_{\Lambda_{\sharp}} \leq \chi_{\Lambda_{\sharp}^{\prime}} \leq \chi_{y_{j^{\prime}}^{\prime \prime}}$ for some $y_{j^{\prime}}^{\prime \prime} \in \Upsilon_{\Lambda}^{\Lambda^{\prime}}$. Thus, it follows from (2.41) and (2.44) that

$$
\begin{aligned}
\left\|\chi_{y} R_{\omega, \Lambda^{\prime}}(z) \chi_{\widehat{\Upsilon}} W_{\Lambda}(\phi) R_{\omega, \Lambda}(z) \chi_{x}\right\| & \\
& \leq c_{d}\left(1+\gamma_{\Re z, d, V_{\mathrm{per}}}^{\prime}\right) \ell^{d-1}\left\|\chi_{y} R_{\omega, \Lambda^{\prime}}(z) \chi_{x^{\prime}}\right\|\left\|\chi_{x^{\prime}} R_{\omega, \Lambda}(z) \chi_{x}\right\|
\end{aligned}
$$

for some $x^{\prime} \in \Upsilon_{\Lambda}^{\Lambda^{\prime}}$.

Combining (2.38) and (2.46) we conclude that

$$
\begin{aligned}
\left\|\chi_{y} R_{\omega, \Lambda^{\prime}}(z) \chi_{x}\right\| \leq & \left\|\chi_{y} \phi R_{\omega, \Lambda}(z) \chi_{x}\right\| \\
& +\gamma_{z} \ell^{d-1}\left\|\chi_{y} R_{\omega, \Lambda^{\prime}}(z) \chi_{x^{\prime}}\right\|\left\|\chi_{x^{\prime}} R_{\omega, \Lambda}(z) \chi_{x}\right\|
\end{aligned}
$$

for some $x^{\prime} \in \Upsilon_{\Lambda}^{\Lambda^{\prime}}$, where $\gamma_{z}$ is as in (2.28), which yields (2.27). If $y \in \Lambda^{\prime} \backslash \Lambda$, then $\chi_{y} \phi=0$, and we get (2.29). If $y \in \Lambda$, using $0 \leq \phi \leq 1$ we get (2.30).

If $z \in \sigma\left(H_{\omega, \Lambda^{\prime}}\right) \backslash \sigma\left(H_{\omega, \Lambda}\right)$, for all have $\varepsilon \neq 0$ we have $z+i \varepsilon \notin \sigma\left(H_{\omega, \Lambda}\right) \cup \sigma\left(H_{\omega, \Lambda^{\prime}}\right)$, and the lemma holds for $z+i \varepsilon$. The lemma then follows for $z$ in view of (2.25).

Lemma 2.5. Consider a box $\Lambda=\Lambda_{\ell} \subset \Lambda^{\prime}$, where $\Lambda^{\prime}$ is either a finite box or $\mathbb{R}^{d}$. Let $\psi$ be a generalized eigenfunction of $H_{\omega, \Lambda^{\prime}}$ with generalized eigenvalue $E \in \mathbb{R} \backslash \sigma\left(H_{\omega, \Lambda}\right)$. Then for every $x \in \Lambda$ with $\Lambda_{\delta_{+}+3}(x) \cap \Lambda^{\prime} \subset \Lambda$, we can find $x^{\prime} \in \Upsilon_{\Lambda}^{\Lambda^{\prime}}$ such that

$$
\left\|\chi_{x} \psi\right\| \leq \gamma_{E} \ell^{d-1}\left\|\chi_{x^{\prime}} R_{\omega, \Lambda}(E) \chi_{x}\right\|\left\|\chi_{x^{\prime}} \psi\right\| .
$$

Proof. Let $\phi=\phi_{\Lambda}^{\Lambda^{\prime}}$ be the function in the proof of the previous lemma (cf. (2.32)-(2.34)). It follows from (2.23) that

$$
\phi \psi=R_{\omega, \Lambda}(E) W_{\Lambda}(\phi) \psi
$$

Thus, given $x \in \Lambda$ with $\Lambda_{\delta_{+}+3}(x) \cap \Lambda^{\prime} \subset \Lambda$, we have

$$
\left\|\chi_{x} \psi\right\|=\left\|\chi_{x} \phi \psi\right\|=\left\|\chi_{x} R_{\omega, \Lambda}(E) W_{\Lambda}(\phi) \psi\right\| .
$$

Proceeding as in (2.41)-(2.46) we get (2.48).

\section{Preamble to the multiscale analysis}

We fix a generalized Anderson Hamiltonian $H_{\omega}$.

\subsection{Good boxes and free sites}

A finite box will be called 'good' at an energy $E$ when the finite volume resolvent is not too big and exhibits exponential decay. As in [Bo, BoK, GHK2], we will also require 'free sites'.

Given a box $\Lambda$, a subset $S \subset \widetilde{\Lambda}$, and $\boldsymbol{t}_{S}=\left\{t_{\zeta}\right\}_{\zeta \in S} \in[0,1]^{S}$, we set

$$
H_{\omega, t_{S}, \Lambda}:=H_{0, \Lambda}+V_{\omega, t_{S}, \Lambda} \quad \text { on } \mathrm{L}^{2}(\Lambda),
$$


where $V_{\omega, t_{S}, \Lambda}=\chi_{\Lambda} V_{\omega_{\Lambda}, t_{S}}$ with

$$
V_{\omega_{\Lambda}, t_{S}}(x):=V_{\omega_{\Lambda \backslash S}}(x)+V_{t_{S}}(x)=\sum_{\zeta \in \widetilde{\Lambda} \backslash S} \omega_{\zeta} u_{\zeta}(x)+\sum_{\zeta \in S} t_{\zeta} u_{\zeta}(x) .
$$

$R_{\boldsymbol{\omega}, t_{S}, \Lambda}(z)$ will denote the corresponding finite volume resolvent.

Definition 3.1. Consider a configuration $\omega \in \Omega$, an energy $E \in \mathbb{C}$, a rate of decay $m>0$, $0<\varsigma<1$, and $S \subset \widetilde{\Lambda}_{L}$. A box $\Lambda_{L}$ is said to be $(\omega, E, m, \varsigma, S)$-good if the following holds for all $\boldsymbol{t}_{S} \in[0,1]^{S}$ :

$$
\left\|R_{\boldsymbol{\omega}, t_{S}, \Lambda_{L}}(E)\right\| \leq \mathrm{e}^{L^{1-\varsigma}}
$$

and

$$
\left\|\chi_{x} R_{\omega, t_{S}, \Lambda_{L}}(E) \chi_{y}\right\| \leq \mathrm{e}^{-m\|x-y\|} \quad \text { for all } x, y \in \Lambda_{L} \text { with }\|x-y\| \geq L / 100 .
$$

In this case $S$ consists of $(\omega, E, m, \varsigma)$-free sites for the box $\Lambda_{L}$. If no free sites are specified, i.e., $S=\emptyset$, then $\Lambda_{L}$ is said to be $(\omega, E, m, \varsigma)$-good.

Remark 3.2. Condition (3.4) is stronger than the usual condition in the definition of a good box (cf. [DrK1, CoH1, GK1, K12]), where decay is postulated only from the center of the box to its boundary. We introduce the exponential decay in $\|x-y\|$ for arbitrary $x, y$ in the box, not too close to each other, in order to prove Lemma 3.10, where we will need to consider locations $x$ and $y$ that may be anywhere in a box $\Lambda^{\prime}$. In particular, we will need to consider the case when both $x$ and $y$ are close to the boundary of $\Lambda^{\prime}$. Thus, we will need to apply Lemma 2.4 for boxes $\Lambda \subset \Lambda^{\prime}$ that touch the boundary of $\Lambda^{\prime}$ (i.e., $\left.\partial \Lambda \cap \partial \Lambda^{\prime} \neq \emptyset\right)$. For this reason we defined $\Upsilon_{\Lambda}^{\Lambda^{\prime}}$ in (2.26) in terms of $\partial^{\Lambda^{\prime}} \Lambda$, the boundary of $\Lambda$ in $\Lambda^{\prime}$.

Remark 3.3. It follows from (2.15) and (2.16) that for all $E \in \mathbb{C}$ we have

$$
\left\{\Lambda_{L} \text { is }(E, m, \varsigma, S) \text {-good }\right\}:=\left\{\omega \in \Omega ; \Lambda_{L} \text { is }(\omega, E, m, \varsigma, S) \text {-good }\right\} \in \mathcal{F}_{\Lambda_{L}} .
$$

Moreover, the set

$$
\left\{\left(E, \omega_{\Lambda_{L}}\right) \in \mathbb{R} \times \Omega_{\Lambda_{L}} ; \Lambda_{L} \text { is }(\omega, E, m, \varsigma, S) \text {-good }\right\}
$$

is closed in $\mathbb{R} \times \Omega_{\Lambda_{L}}$, and hence jointly measurable in $\left(E, \omega_{\Lambda_{L}}\right)$.

Definition 3.4. Consider an energy $E \in \mathbb{R}$, a rate of decay $m>0$, and numbers $0<\varsigma<1$ and $p>0$. A scale $L>0$ is called $(E, m, \varsigma, p)$-good if for every $x \in \mathbb{R}^{d}$ we have

$$
\mathbb{P}\left\{\Lambda_{L}(x) \text { is }(E, m, \varsigma) \text {-good }\right\} \geq 1-L^{-p d} .
$$

If a box $\Lambda_{L}$ is $(\omega, E, m, \varsigma)$-good, then it is just as good for energies $E^{\prime}$ such that $\left|E^{\prime}-E\right| \leq \mathrm{e}^{-c L}$, the precise statement being given in the following definition and lemma. 
Definition 3.5. Consider a configuration $\omega \in \Omega$, an energy $E \in \mathbb{C}$, a rate of decay $m>0$, and $0<\varsigma<1$. A box $\Lambda_{L}$ is said to be $(\omega, E, m, \varsigma)$-jgood (just as good) if

$$
\left\|R_{\omega, \Lambda_{L}}(E)\right\| \leq 2 \mathrm{e}^{L^{1-\varsigma}}
$$

and

$$
\left\|\chi_{x} R_{\omega, \Lambda_{L}}(E) \chi_{y}\right\| \leq 2 \mathrm{e}^{-m\|x-y\|} \quad \text { for all } x, y \in \Lambda_{L} \text { with }\|x-y\| \geq L / 100 .
$$

Lemma 3.6. Let $\omega \in \Omega, E \in \mathbb{C}, 0<\tau<\varsigma<1$. Suppose the box $\Lambda_{L}$ is $(\omega, E, m, \varsigma)$ good with a rate of decay $m \geq L^{-\tau}$. Then, if $L \geq \widetilde{L}_{\varsigma, \tau}$, the box $\Lambda_{L}$ is $\left(\omega, E^{\prime}, m, \varsigma\right)$-jgood for all energies $E^{\prime} \in \mathbb{C}$ such that $\left|E^{\prime}-E\right| \leq \mathrm{e}^{-2 m L}$.

Proof. By the resolvent identity,

$$
R_{\omega, \Lambda_{L}}\left(E^{\prime}\right)=R_{\omega, \Lambda_{L}}(E)-\left(E^{\prime}-E\right) R_{\omega, \Lambda_{L}}(E) R_{\omega, \Lambda_{L}}\left(E^{\prime}\right) .
$$

Thus, for $\left|E^{\prime}-E\right| \leq \mathrm{e}^{-2 m L}$, we get

$$
\left\|R_{\omega, \Lambda_{L}}\left(E^{\prime}\right)\right\| \leq \mathrm{e}^{L^{1-\varsigma}}+\mathrm{e}^{-2 m L} \mathrm{e}^{L^{1-\varsigma}}\left\|R_{\omega, \Lambda_{L}}\left(E^{\prime}\right)\right\|
$$

Since $0<\tau<\varsigma<1$, (3.8) follows.

Similarly, using also (3.8), given $x, y \in \Lambda_{L}$ with $\|x-y\| \geq L / 100$, we have

$$
\left\|\chi_{x} R_{\omega, \Lambda_{L}}\left(E^{\prime}\right) \chi_{y}\right\| \leq \mathrm{e}^{-m\|x-y\|}+2 \mathrm{e}^{-2 m L} \mathrm{e}^{2 L^{1-\zeta}},
$$

and (3.9) follows.

We also need the following variant of Lemma 3.6; the proof is almost identical.

Lemma 3.7. Let $\omega \in \Omega, E \in \mathbb{C}, 0<\varsigma<1$, and $0<\widetilde{m}<m$. Suppose the box $\Lambda_{L}$ is $(\boldsymbol{\omega}, E, m, \varsigma)$-good. Then, if $L \geq \widetilde{L}_{\zeta, \widetilde{m}}$, given $E^{\prime} \in \mathbb{C}$ with $\left|E^{\prime}-E\right| \leq \mathrm{e}^{-m_{1} L}$, where $m_{1} \in[\tilde{m}, m]$, the box $\Lambda_{L}$ is $\left(\boldsymbol{\omega}, E^{\prime}, m_{2}, \varsigma\right)$-jgood with

$$
m_{2}=m_{1}\left(1-C \tilde{m}^{-1} L^{-\varsigma}\right) .
$$

The following definition will be needed only for real energies.

Definition 3.8. Consider an energy $E \in \mathbb{R}$, a rate of decay $m>0$, and numbers $0<$ $\varsigma, \varsigma^{\prime}<1$ and $p>0$.

(i) Given a box $\Lambda_{L}$, a subset $S \subset \widetilde{\Lambda}_{L}$ is called $\varsigma^{\prime}$-abundant if

$$
\#\left(S \cap \Lambda_{L / 5}\right) \geq L^{\left(1-\varsigma^{\prime}\right) d} \quad \text { for all boxes } \Lambda_{L / 5} \subset \Lambda_{L} .
$$

(ii) Given a box $\Lambda_{L}$, an event $\mathcal{C}$ is said to be $\left(\Lambda_{L}, E, m, \varsigma, \varsigma^{\prime}\right)$-adapted if there exists a $\varsigma^{\prime}$-abundant subset $S_{\mathcal{C}} \subset \widetilde{\Lambda}_{L}$ such that $\mathcal{C} \in \mathcal{F}_{\Lambda_{L} \backslash S_{\mathcal{C}}}$ and $\Lambda_{L}$ is $\left(\omega, E, m, \zeta, S_{\mathcal{C}}\right)$ good for all $\omega \in \mathcal{C}$. In this case $\mathcal{C}$ will also be called $\left(\Lambda_{L}, E, m, \varsigma, \varsigma^{\prime}, S_{\mathcal{C}}\right)$-adapted. 
(iii) Given a box $\Lambda_{L}$, an event $\mathcal{E}$ is called $\left(\Lambda_{L}, E, m, \varsigma, \varsigma^{\prime}\right)$-extra good if it is the disjoint union of a finite number of $\left(\Lambda_{L}, E, m, \varsigma, \varsigma^{\prime}\right)$-adapted events, i.e., there exist disjoint $\left(\Lambda_{L}, E, m, \varsigma, \varsigma^{\prime}\right)$-adapted events $\left\{\mathcal{C}_{i}\right\}_{i=1, \ldots, I}$ such that

$$
\mathcal{E}=\bigsqcup_{i=1}^{I} \mathcal{C}_{i}
$$

(iv) A scale $L>0$ is called ( $\left.E, m, \varsigma, \varsigma^{\prime}, p\right)$-extra good if for every $x \in \mathbb{R}^{d}$ there exists a $\left(\Lambda_{L}(x), E, m, \varsigma, \varsigma^{\prime}\right)$-extra good event $\mathcal{E}_{L, x}$ such that

$$
\mathbb{P}\left\{\mathcal{E}_{L, x}\right\} \geq 1-L^{-p d} .
$$

If a scale $L$ is $\left(E, m, \varsigma, \varsigma^{\prime}, p\right)$-extra good, it is clearly also $(E, m, \varsigma, p)$-good.

\subsection{Tools for the multiscale analysis}

We now combine Lemmas 2.4 and 2.5 with good boxes to obtain crucial tools for the multiscale analysis. In Lemmas 3.9 and 3.10 we will not know a priori that $E \notin \sigma\left(H_{\omega, \Lambda}\right)$, and we will apply Lemma 2.4 with the notation given in (2.25).

Lemma 3.9. Fix a configuration $\omega \in \Omega$ and an energy $E \in \mathbb{C}$. Let $\Lambda$ be either $\mathbb{R}^{d}$ or $a$ box $\Lambda_{L}$. Consider a scale $\ell$, with $\ell<L / 6$ if $\Lambda=\Lambda_{L}$, numbers $0<\tau<\varsigma<1$, and $m \geq \ell^{-\tau}$. Let $\Theta \subset \Lambda$ be such that for all $x \in \Lambda \backslash \Theta$ there exists an $(\omega, E, m, \varsigma)$-good box, denoted by $\Lambda_{\ell}^{(x)}$, such that $\Lambda_{\ell}^{(x)} \subset \Lambda$ with $\Lambda_{\ell / 5}(x) \cap \Lambda \subset \Lambda_{\ell}^{(x)}$. Then there exists $a$ constant $C=C_{d, V_{\mathrm{per}}, E}$, locally bounded in $E$, such that setting

$$
m^{\prime}=m\left(1-C(\log \ell) \ell^{\tau-1}\right)
$$

the following holds:

(i) For all $x, y \in \Lambda$ with $x \notin \Theta$ we have

$$
\left\|\chi_{y} R_{\omega, \Lambda}(E) \chi_{x}\right\| \leq\left\|\chi_{y} \chi_{\Lambda_{\ell}^{(x, \Lambda, 1 / 2)}} R_{\omega, \Lambda}^{(x)}(E) \chi_{x}\right\|+\mathrm{e}^{-m^{\prime}\left\|x-x_{1}\right\|}\left\|\chi_{y} R_{\omega, \Lambda}(E) \chi_{x_{1}}\right\|,
$$

for some $x_{1} \in \Upsilon_{\Lambda_{\ell}^{(x)}}^{\Lambda}$, so in particular

$$
\ell / 11 \leq\left\|x-x_{1}\right\| \leq \ell .
$$

(ii) Let $x, y \in \Lambda$ with $x \notin \Theta$ and $\|x-y\| \geq \ell$. Then

$$
\left\|\chi_{y} R_{\omega, \Lambda}(E) \chi_{x}\right\| \leq \mathrm{e}^{-m^{\prime}\left\|x-x^{\prime}\right\|}\left\|\chi_{y} R_{\omega, \Lambda}(E) \chi_{x^{\prime}}\right\|
$$

for some $x^{\prime} \in \Lambda$ such that either $x^{\prime} \in \Theta$ or $\left\|x^{\prime}-y\right\|<\ell$, i.e.,

$$
x^{\prime} \in \Theta \cup \Lambda_{2 \ell}(y) \text {. }
$$


(iii) Suppose $E \in \mathbb{R}$ and $\psi$ is a generalized eigenfunction of $H_{\omega, \Lambda}$ with generalized eigenvalue $E^{\prime} \in\left[E-\mathrm{e}^{-2 m \ell}, E+\mathrm{e}^{-2 m \ell}\right]$. Then for all $x \in \Lambda \backslash \Theta$ we have

$$
\left\|\chi_{x} \psi\right\| \leq \mathrm{e}^{-m^{\prime}\left\|x-x^{\prime}\right\|}\left\|\chi_{x^{\prime}} \psi\right\| \leq \mathrm{e}^{-\frac{m^{\prime}}{11} \ell}\left\|\chi_{x^{\prime}} \psi\right\| \quad \text { for some } x^{\prime} \in \Upsilon_{\Lambda_{\ell}^{(x)}}^{\Lambda}
$$

and also

$$
\left\|\chi_{x} \psi\right\| \leq \mathrm{e}^{-m^{\prime}\left\|x-x^{\prime \prime}\right\|}\left\|\chi_{x^{\prime \prime}} \psi\right\| \leq \mathrm{e}^{-m^{\prime} \operatorname{dist}\{x, \Theta\}}\left\|\chi_{x^{\prime \prime}} \psi\right\| \quad \text { for some } x^{\prime \prime} \in \Theta \text {. }
$$

If $E^{\prime}=E$, then (3.22) and (3.23) hold with $m$ substituted for $m^{\prime}$.

Proof. (i) Since $x \notin \Theta$, we use the existence of the good box $\Lambda_{\ell}^{(x)}$ and apply (2.27) to get

$$
\begin{aligned}
& \left\|\chi_{y} R_{\boldsymbol{\omega}, \Lambda}(E) \chi_{x}\right\| \\
& \quad \leq\left\|\chi_{y} \chi_{\Lambda_{\ell}^{(x, \Lambda, 1 / 2)}} R_{\omega, \Lambda_{\ell}^{(x)}}(E) \chi_{x}\right\|+\gamma_{E} \ell^{d-1} \mathrm{e}^{-m\left\|x-x_{1}\right\|}\left\|\chi_{y} R_{\boldsymbol{\omega}, \Lambda}(E) \chi_{x_{1}}\right\|
\end{aligned}
$$

for some $x_{1} \in \Upsilon_{\Lambda_{\ell}^{(x)}}^{\Lambda}$, so $\ell / 10-\left(\delta_{+}+1\right) / 2 \leq\left\|x-x_{1}\right\| \leq \ell-\left(\delta_{+}+1\right) / 2$, hence (3.19) holds, and we have (3.18) with (3.17).

(ii) Since $x \notin \Theta$ and $\|x-y\| \geq \ell$, we apply (3.18) repeatedly to get

$$
\left\|\chi_{y} R_{\omega, \Lambda}(E) \chi_{x}\right\| \leq \mathrm{e}^{-m^{\prime} \sum_{i=1}^{n}\left\|x_{i-1}-x_{i}\right\|}\left\|\chi_{y} R_{\omega, \Lambda}(E) \chi_{x_{n}}\right\|
$$

with $x_{0}=x$ and $x_{i} \in \Upsilon_{\Lambda_{\ell}^{\left(x_{i-1}\right)}}^{\Lambda}, i=1, \ldots, n$, where $n \in \mathbb{N}$ is such that $x_{i} \notin \Theta$ and $\left\|x_{i}-y\right\| \geq \ell$ for $i=0,1, \ldots, n-1$, and either $x_{n} \in \Theta$ or $\left\|x_{n}-y\right\|<\ell$. Since $\left\|x_{0}-x_{n}\right\| \leq \sum_{i=1}^{n}\left\|x_{i-1}-x_{i}\right\|$, (3.20) follows.

(iii) It follows from Lemma 3.6 that for all $x \notin \Theta$ the box $\Lambda_{\ell}^{(x)}$ is $\left(\omega, E^{\prime}, m, \varsigma\right)$-jgood. Thus, given $x \notin \Theta$, we apply Lemma 2.5 with the box $\Lambda_{\ell}^{(x)}$ to get (3.22). To prove (3.23), we proceed similarly to the proof of (3.20), applying Lemma 2.5 repeatedly.

Note that in (iii) the constant $C$ in (3.17) depends on $E^{\prime}$. Since $\left|E^{\prime}-E\right| \leq 1$, we can fix a constant $C=C_{E, d, V_{\text {per }}}$, locally bounded in $E$, that works for all the conclusions of the lemma.

The following lemma will play an important role in the multiscale analysis. We use the notation given in (2.19).

Lemma 3.10. Fix a configuration $\omega \in \Omega$ and an energy $E \in \mathbb{C}$. Consider a box $\Lambda=\Lambda_{L}$ and let $\varsigma, \rho, \kappa, \tau \in] 0,1\left[, \ell=L^{\rho}, m \geq \ell^{-\tau}\right.$, and $K, K^{\prime} \in \mathbb{N}$, where

$$
\kappa \zeta>\tau \rho \text {. }
$$

Suppose there exist $\Theta=\bigsqcup_{j=1}^{K} \Theta_{j} \subset \Lambda$ satisfying the following conditions:

(i) There exist disjoint boxes $\Lambda_{j}=\Lambda_{L_{j}}\left(y_{j}\right) \subset \Lambda$ with $L^{\kappa} \leq L_{j} \leq K^{\prime} L^{\kappa}, j=1, \ldots, K$, such that

$$
\begin{aligned}
& \Theta_{j} \subset \Lambda_{j}^{\left(\Lambda, L^{\kappa} / 10\right)} \\
& \left\|R_{\boldsymbol{\omega}, \Lambda_{j}}(E)\right\| \leq \mathrm{e}^{L^{\kappa(1-\varsigma)}} .
\end{aligned}
$$


(ii) For all $x \in \Lambda \backslash \Theta$ there exists an $(\omega, E, m, \varsigma)$-good box $\Lambda_{\ell}^{(x)} \subset \Lambda$ such that $\Lambda_{\ell / 5}(x) \cap$ $\Lambda \subset \Lambda_{\ell}^{(x)}$.

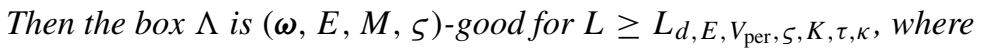

$$
M \geq m\left(1-C\left(L^{\kappa-1}+L^{\rho \tau-\kappa \zeta}\right)\right) \geq L^{-\tau}
$$

and $C=C_{E, d, V_{\mathrm{per}}, K, K^{\prime}}$ is locally bounded in $E$.

Proof. We start by proving (3.3) for $\Lambda$. Since $H_{\omega, \Lambda}$ has discrete spectrum, there exists $\varepsilon>0$ such that $E^{\prime} \notin \sigma\left(H_{\omega, \Lambda}\right)$ if $0<\left|E^{\prime}-E\right|<\varepsilon$. We take $\varepsilon \leq \mathrm{e}^{-2 m \ell}$, so the boxes $\Lambda_{\ell}^{(x)}$ given in condition (ii) are $\left(\omega, E^{\prime}, m, \varsigma\right)$-jgood by Lemma 3.6, and small enough such that it follows from (3.28) that

$$
\left\|R_{\omega, \Lambda_{j}}\left(E^{\prime}\right)\right\| \leq 2 \mathrm{e}^{L^{K(1-\varsigma)}} \quad \text { for } j=1, \ldots, K .
$$

We will estimate $\left\|R_{\omega, \Lambda}\left(E^{\prime}\right)\right\|$ for $0<\left|E^{\prime}-E\right|<\varepsilon$. Suppose either $x$ or $y$ is not in $\Theta$, say $x \notin \Theta$. In this case we apply Lemma 3.9(i). It follows from (3.18), appropriately modified for jgood boxes, Definition 3.5, and (3.19), that

$$
\begin{aligned}
\left\|\chi_{y} R_{\omega, \Lambda}\left(E^{\prime}\right) \chi_{x}\right\| & \leq 2 \mathrm{e}^{\ell^{1-\varsigma}}+2 \mathrm{e}^{-m^{\prime} \ell / 11}\left\|R_{\omega, \Lambda}\left(E^{\prime}\right)\right\| \leq 2 \mathrm{e}^{\ell^{1-\varsigma}}+2 \mathrm{e}^{-\frac{1}{15} \ell^{1-\tau}}\left\|R_{\omega, \Lambda}\left(E^{\prime}\right)\right\| \\
& \leq 2 \mathrm{e}^{\ell^{1-\varsigma}}+\frac{1}{2} L^{-2 d}\left\|R_{\omega, \Lambda}\left(E^{\prime}\right)\right\|
\end{aligned}
$$

for large $L$. If $x \in \Theta$ and $y \notin \Theta$ we use $\left\|\chi_{y} R_{\omega, \Lambda}\left(E^{\prime}\right) \chi_{x}\right\|=\left\|\chi_{x} R_{\omega, \Lambda}\left(E^{\prime}\right) \chi_{y}\right\|$ to get (3.31). Suppose now $x, y \in \Theta$, say $x \in \Theta_{s}$. Then we apply (2.27) with the box $\Lambda_{s}$, and use (3.30), getting

$$
\left\|\chi_{y} R_{\omega, \Lambda}\left(E^{\prime}\right) \chi_{x}\right\| \leq 2 \mathrm{e}^{L^{K(1-\varsigma)}}+2 \gamma\left(K^{\prime} L^{\kappa}\right)^{d-1} \mathrm{e}^{L^{k(1-\varsigma)}}\left\|\chi_{y} R_{\omega, \Lambda}\left(E^{\prime}\right) \chi_{x_{0}}\right\|,
$$

where $x_{0} \in \Upsilon_{\Lambda_{s}}^{\Lambda}$ and $\gamma=\gamma_{E+1}$. Note that (3.27) implies $\operatorname{dist}\left\{x_{0}, \Theta\right\} \geq L^{\kappa} / 11$; in particular, $\left\|x_{0}-y\right\| \geq L^{\kappa} / 11$ as $y \in \Theta$. We can now use Lemma 3.9(ii), with $m^{\prime} / 2$ replacing $m^{\prime}$ in (3.20) to compensate for using jgood boxes instead of good ones, to conclude that

$$
\begin{aligned}
\left\|\chi_{y} R_{\omega, \Lambda}\left(E^{\prime}\right) \chi_{x_{0}}\right\| & \leq \mathrm{e}^{-m^{\prime} / 2\left\|x_{0}-x^{\prime}\right\|}\left\|\chi_{y} R_{\omega, \Lambda}\left(E^{\prime}\right) \chi_{x^{\prime}}\right\| \\
& \leq \mathrm{e}^{-m^{\prime} L^{\kappa} / 30}\left\|\chi_{y} R_{\omega, \Lambda}\left(E^{\prime}\right) \chi_{x^{\prime}}\right\|,
\end{aligned}
$$

where $x^{\prime}$ satisfies (3.21), so $\left\|x_{0}-x^{\prime}\right\| \geq L^{\kappa} / 11-\ell>L^{\kappa} / 15$ for large $L$. From (3.32), (3.33), and (3.26), we conclude that, for large $L$, we have

$$
\begin{aligned}
\left\|\chi_{y} R_{\omega, \Lambda}\left(E^{\prime}\right) \chi_{x}\right\| & \leq 2 \mathrm{e}^{L^{K(1-\varsigma)}}+2 \gamma\left(K^{\prime} L^{\kappa}\right)^{d-1} \mathrm{e}^{L^{K(1-\varsigma)}} \mathrm{e}^{-\frac{1}{60} \ell^{-\tau} L^{\kappa}}\left\|R_{\omega, \Lambda}\left(E^{\prime}\right)\right\| \\
& \leq 2 \mathrm{e}^{L^{K(1-\varsigma)}}+\frac{1}{2} L^{-2 d}\left\|R_{\omega, \Lambda}\left(E^{\prime}\right)\right\| .
\end{aligned}
$$

Combining (3.31) and (3.34) we get

$$
\begin{aligned}
\left\|R_{\boldsymbol{\omega}, \Lambda}\left(E^{\prime}\right)\right\| & \leq L^{2 d}\left\{2 \mathrm{e}^{L^{\kappa(1-\varsigma)}}+\frac{1}{2} L^{-2 d}\left\|R_{\boldsymbol{\omega}, \Lambda}\left(E^{\prime}\right)\right\|\right\} \\
& \leq 2 L^{2 d} \mathrm{e}^{L^{\kappa(1-\varsigma)}}+\frac{1}{2}\left\|R_{\boldsymbol{\omega}, \Lambda}\left(E^{\prime}\right)\right\|,
\end{aligned}
$$


and hence, for large $L$,

$$
\left\|R_{\boldsymbol{\omega}, \Lambda}\left(E^{\prime}\right)\right\| \leq 2 L^{2 d} \mathrm{e}^{L^{k(1-\varsigma)}} \leq \mathrm{e}^{L^{1-\varsigma}} .
$$

We now conclude that for large $L$ we have

$$
\left\|R_{\omega, \Lambda}(E)\right\|=\lim _{E^{\prime} \rightarrow E}\left\|R_{\omega, \Lambda}\left(E^{\prime}\right)\right\| \leq \mathrm{e}^{L^{1-\varsigma}} .
$$

To finish the proof, we need to prove (3.4) for the box $\Lambda$.

Sublemma 3.11. Given $s \in\{1,2, \ldots, K\}$, let $x, y \in \Lambda$ with $x \in \Theta_{s}$ and $\|x-y\| \geq L_{s}$. Then there exist $x^{(0)} \in \Upsilon_{\Lambda_{s}}^{\Lambda}$ and $x^{\prime} \in \Lambda$, with $x^{\prime}$ satisfying (3.21) and

$$
\frac{1}{11} L^{\kappa} \leq\left\|x^{(0)}-x\right\| \leq L_{s}-\frac{1}{10} L^{\kappa} \quad \text { and } \quad\left\|x^{(0)}-y\right\| \geq \frac{1}{10} L^{\kappa},
$$

such that

$$
\left\|\chi_{y} R_{\omega, \Lambda}(E) \chi_{x}\right\| \leq \mathrm{e}^{-m^{\prime \prime}\left\|x^{(0)}-x^{\prime}\right\|}\left\|\chi_{y} R_{\omega, \Lambda}(E) \chi_{x^{\prime}}\right\|,
$$

where

$$
m^{\prime \prime}=m^{\prime}\left(1-C \ell^{\tau} L^{-\kappa \varsigma}\right) \quad \text { with } C=C_{E, d, V_{\mathrm{per}}, K^{\prime}} \text { locally bounded in } E .
$$

Proof. Let $x, y \in \Lambda$ with $x \in \Theta_{s}$ and $\|x-y\| \geq L_{s}$. We proceed as in (3.32) and (3.33) (note that we are now working at energy $E$, so we have (3.28) and condition (ii) holds), getting

$$
\begin{aligned}
\left\|\chi_{y} R_{\omega, \Lambda}(E) \chi_{x}\right\| & \leq \gamma_{E}\left(K^{\prime} L^{\kappa}\right)^{d-1} \mathrm{e}^{L^{k(1-\varsigma)}}\left\|\chi_{y} R_{\omega, \Lambda}(E) \chi_{x^{(0)}}\right\| \\
& \leq \gamma_{E}\left(K^{\prime} L^{\kappa}\right)^{d-1} \mathrm{e}^{L^{k(1-\varsigma)}} \mathrm{e}^{-m^{\prime}\left\|x^{(0)}-x^{\prime}\right\|}\left\|\chi_{y} R_{\omega, \Lambda}(E) \chi_{x^{\prime}}\right\| \\
& \leq \mathrm{e}^{-m^{\prime \prime}\left\|x^{(0)}-x^{\prime}\right\|}\left\|\chi_{y} R_{\omega, \Lambda}(E) \chi_{x^{\prime}}\right\|,
\end{aligned}
$$

where $x^{(0)} \in \Upsilon_{\Lambda_{s}}^{\Lambda}$, so we have (3.38), and $x^{\prime} \in \Lambda$ satisfies (3.21), so $\left\|x^{(0)}-x^{\prime}\right\| \geq$ $L^{\kappa} / 11-\ell>L^{\kappa} / 15$, and thus $m^{\prime \prime}$ is as in (3.40).

Now let $x, y \in \Lambda$ with $\|x-y\| \geq L / 100 \geq K^{\prime} L^{\kappa}$. If $x \notin \Theta$, we apply Lemma 3.9(ii), obtaining $x^{\prime}$ satisfying (3.21). If $\left\|x^{\prime}-y\right\|<K^{\prime} L^{\kappa}$, we stop. Otherwise we then start from $x^{\prime}$ and apply Sublemma 3.11 repeatedly, until we get

$$
\left\|\chi_{y} R_{\omega, \Lambda}(E) \chi_{x}\right\| \leq \mathrm{e}^{-m^{\prime \prime} \sum_{i=1}^{n}\left\|x_{i-1}^{(0)}-x_{i}\right\|}\left\|\chi_{y} R_{\omega, \Lambda}(E) \chi_{x_{n}}\right\|,
$$

where $x=x_{0}=x_{0}^{(0)}, x_{1}=x^{\prime}, x_{i-1}^{(0)}$ and $x_{i}$ correspond to $x^{(0)}$ and $x^{\prime}$ in Sublemma 3.11 for $x_{i-1}, y$ for $i=2, \ldots, n$, and $n \in \mathbb{N}$ is such that $\left\|x_{i}-y\right\| \geq K^{\prime} L^{\kappa}$ (and hence $\left.x_{i} \in \Theta\right)$ for $i=1, \ldots, n-1$, and $\left\|x_{n}-y\right\|<K^{\prime} L^{\kappa}$. If $x \in \Theta$, we start directly with Sublemma 3.11 obtaining also (3.42) but with $x=x_{0}$, and $x_{0}^{(0)}$ and $x_{1}$ corresponding to $x^{(0)}$ and $x^{\prime}$ in Sublemma 3.11 for $x_{0}$ and $y$.

Now let us choose distinct $j_{0}, j_{1}, \ldots, j_{r} \in\{0,1, \ldots, K+1\}$, where $0 \leq r \leq K+1$, as follows: 
(a) If $x \notin \Theta$, we set $j_{0}=0$ and $\Lambda_{0}=\Theta_{0}=\{x\}$. If $x \in \Theta, j_{0}$ is determined by $x \in \Theta_{j_{0}}$. Set also $\Theta_{K+1}=\left\{x_{n}\right\}$.

(b) Pick $j_{1} \neq j_{0}$ such that for some $i_{1} \in\{1, \ldots, n\}$ we have $x_{i_{1}-1}^{(0)} \in \Lambda_{j_{0}}$ and $x_{i_{1}} \in \Theta_{j_{1}}$.

(c) Given $j_{0}, j_{1}, \ldots, j_{s}$, then if $i_{s}=n, r=s$, so stop. If not, pick $j_{s+1} \notin\left\{j_{0}, j_{1}, \ldots, j_{s}\right\}$ such that that for some $i_{s+1} \in\{1, \ldots, n\}$ we have $x_{i_{s+1}-1}^{(0)} \in \Lambda_{j_{s}}$ and $x_{i_{s+1}} \in \Theta_{j_{s+1}}$.

It then follows from (3.42) that

$$
\left\|\chi_{y} R_{\omega, \Lambda}(E) \chi_{x}\right\| \leq \mathrm{e}^{-m^{\prime \prime} \sum_{s=1}^{r}\left\|x_{i_{s}-1}^{(0)}-x_{i s}\right\|}\left\|R_{\boldsymbol{\omega}, \Lambda}(E)\right\| .
$$

By our construction,

$\sum_{s=1}^{r}\left\|x_{i_{s}-1}^{(0)}-x_{i_{s}}\right\| \geq \sum_{s=1}^{r} \operatorname{dist}\left\{\Lambda_{s-1}, \Lambda_{s}\right\} \geq\left\|x-x_{n}\right\|-K K^{\prime} L^{\kappa} \geq\|x-y\|-\left(K K^{\prime}+1\right) L^{\kappa}$.

It follows, using also (3.37), that

$$
\left\|\chi_{y} R_{\omega, \Lambda}(E) \chi_{x}\right\| \leq \mathrm{e}^{-m^{\prime \prime}\left(\|x-y\|-\left(K K^{\prime}+1\right) L^{\kappa}\right)} \mathrm{e}^{L^{1-\varsigma}} \leq \mathrm{e}^{-M(\|x-y\|)},
$$

where

$$
M=m^{\prime \prime}\left(1-C\left(L^{\kappa} L^{-1}+\ell^{\tau} L^{-\varsigma}\right)\right),
$$

with a constant $C=C_{E, d, V_{\mathrm{per}}, K, K^{\prime}}$ locally bounded in $E$.

The lemma is proved.

\subsection{Suitable coverings of boxes and annuli}

\subsubsection{Suitable coverings of boxes}

Definition 3.12. Given scales $\ell<L$, a suitable $\ell$-covering of a box $\Lambda_{L}(x)$ is a collection of boxes $\Lambda_{\ell}$ of the form

$$
\mathcal{G}_{\Lambda_{L}(x)}^{(\ell)}=\left\{\Lambda_{\ell}(r)\right\}_{r \in \mathbb{G}_{\Lambda_{L}(x)}^{(\ell)}},
$$

where

$$
\mathbb{G}_{\Lambda_{L}(x)}^{(\ell)}:=\left\{x+\alpha \ell \mathbb{Z}^{d}\right\} \cap \Lambda_{L}(x) \quad \text { with } \quad \alpha \in\left[\frac{3}{5}, \frac{4}{5}\right] \cap\left\{\frac{L-\ell}{2 \ell n} ; n \in \mathbb{N}\right\} .
$$

Lemma 3.13. Let $\ell \leq L / 6$. Then every box $\Lambda_{L}(x)$ has a suitable $\ell$-covering, and for any suitable $\ell$-covering $\mathcal{G}_{\Lambda_{L}(x)}^{(\ell)}$ of $\Lambda_{L}(x)$ we have

$$
\begin{aligned}
& \Lambda_{L}(x)=\bigcup_{r \in \mathbb{G}_{\Lambda_{L}(x)}^{(\ell)}} \Lambda_{\ell}(r), \\
& \text { for each } y \in \Lambda_{L}(x) \text { there is } r \in \mathbb{G}_{\Lambda_{L}(x)}^{(\ell)} \text { with } \Lambda_{\ell / 5}(y) \cap \Lambda_{L}(x) \subset \Lambda_{\ell}(r), \\
& \Lambda_{\ell / 5}(r) \cap \Lambda_{\ell}\left(r^{\prime}\right)=\emptyset \quad \text { for all } r, r^{\prime} \in x+\alpha \ell \mathbb{Z}^{d}, r \neq r^{\prime}, \\
& \left(\frac{L}{\ell}\right)^{d} \leq \# \mathbb{G}_{\Lambda_{L}(x)}^{(\ell)}=\left(\frac{L-\ell}{\alpha \ell}+1\right)^{d} \leq\left(\frac{2 L}{\ell}\right)^{d} .
\end{aligned}
$$


Moreover, given $y \in x+\alpha \ell \mathbb{Z}^{d}$ and $n \in \mathbb{N}$, it follows that

$$
\Lambda_{(2 n \alpha+1) \ell}(y)=\bigcup_{r \in\left\{x+\alpha \ell \mathbb{Z}^{d}\right\} \cap \Lambda_{(2 n \alpha+1) \ell}(y)} \Lambda_{\ell}(r),
$$

and $\left\{\Lambda_{\ell}(r)\right\}_{r \in\left\{x+\alpha \ell \mathbb{Z}^{d}\right\} \cap \Lambda_{(2 n \alpha+1) \ell}(y)}$ is a suitable $\ell$-covering of the box $\Lambda_{(2 n \alpha+1) \ell}(y)$. In particular,

$$
\text { for each } y \in \mathbb{Z}^{d} \text { there is } r \in x+\alpha \ell \mathbb{Z}^{d} \text { with } \Lambda_{\ell / 5}(y) \subset \Lambda_{\ell}(r) .
$$

Proof. It suffices to note that $\ell \leq L / 6$ ensures [(3)/5, 4/5] $\{L-\ell / 2 \ell n ; n \in \mathbb{N}\} \neq \emptyset$, $\alpha \leq 4 / 5$ gives (3.50) and (3.54), and $\alpha \geq 3 / 5$ yields (3.51).

To fix ideas we make the following definition.

Definition 3.14. The standard $\ell$-covering of a box $\Lambda_{L}(x)$ is the unique suitable $\ell$-covering of $\Lambda_{L}(x)$ with

$$
\alpha=\alpha_{L, \ell}:=\max \left\{[3 / 5,4 / 5] \cap\left\{\frac{L-\ell}{2 \ell n} ; n \in \mathbb{N}\right\}\right\} .
$$

We now consider standard coverings by good boxes.

Definition 3.15. Consider a configuration $\omega \in \Omega$, an energy $E \in \mathbb{R}$, a rate of decay $m>0,0<\varsigma<1$, and $\eta>0$. A box $\Lambda_{L}$ is said to be $(\boldsymbol{\omega}, E, m, \varsigma, \eta)$-pgood (for predecessor of good) if, letting $\ell=L^{1 /(1+\eta)}$, every box $\Lambda_{\ell}$ in the standard $\ell$-covering of $\Lambda_{L}$ is $(\boldsymbol{\omega}, E, m, \varsigma)$-good.

Lemma 3.16. Suppose the box $\Lambda_{L}$ is $(\omega, E, m, \varsigma, \eta)$-pgood for some $\omega \in \Omega, E \in \mathbb{R}$, $m>0,0<\varsigma<1$, and $\eta>0$, set $\ell=L^{1 /(1+\eta)}$, and let $0<\widehat{m} \leq m$. Then, if $L \geq \widehat{L}_{\varsigma, \widetilde{m}, \eta}$, given $m_{1} \in[\widehat{m}, m]$, the box $\Lambda_{L}$ is $\left(\omega, E^{\prime}, M_{1}, \varsigma\right)$-good for all energies $E^{\prime} \in \mathbb{C}$ such that $\left|E^{\prime}-E\right| \leq \mathrm{e}^{-m_{1} \ell}$, where

$$
M_{1}=m_{1}\left(1-C_{d, p, \widehat{m}} L^{-\min \{s, \eta\} /(1+\eta)}\right) .
$$

Proof. Let $\Lambda_{\ell}$ be $(\omega, E, m, \varsigma)$-good and $E^{\prime} \in \mathbb{C}$ with $\left|E^{\prime}-E\right| \leq \mathrm{e}^{-m_{1} \ell}$. It follows from Lemma 3.7 that $\Lambda_{\ell}$ is $\left(\boldsymbol{\omega}, E^{\prime}, m_{2}, \varsigma\right)$-jgood if $\ell \geq \widetilde{\ell}_{5, \widehat{m}}$, with $m_{2}=m_{1}\left(1-C \widehat{m}^{-1} \ell^{-\varsigma}\right)$.

Now suppose $\Lambda=\Lambda_{L}$ is $(\boldsymbol{\omega}, E, m, \varsigma, \eta)$-pgood and $\ell \geq \tilde{\ell}_{5, \widehat{m}}$. We proceed as in Lemma 3.10 (but note that $\Theta=\emptyset$ ). Proceeding as in (3.31) and (3.35), using the fact that every box $\Lambda_{\ell}$ in the standard $\ell$-covering of $\Lambda_{L}$ is $\left(\boldsymbol{\omega}, E^{\prime}, m_{2}, \varsigma\right)$-jgood, we get, for $L$ sufficently large,

$$
\begin{aligned}
\left\|R_{\boldsymbol{\omega}, \Lambda}\left(E^{\prime}\right)\right\| & \leq L^{2 d}\left(2 \mathrm{e}^{\ell^{1-\varsigma}}+\mathrm{e}^{-m_{3} \ell / 11}\left\|R_{\omega, \Lambda}\left(E^{\prime}\right)\right\|\right) \\
& \leq 2 L^{2 d} e^{\ell^{1-\varsigma}}+\frac{1}{2}\left\|R_{\omega, \Lambda}(E)\right\|
\end{aligned}
$$

where $m_{3}=m_{2}\left(1-C_{d, V_{\mathrm{per}}, p, \widehat{m}, \mathcal{I}} \frac{\log \ell}{\ell}\right)$, and hence

$$
\left\|R_{\boldsymbol{\omega}, \Lambda}\left(E^{\prime}\right)\right\| \leq 4 L^{2 d} e^{\ell^{1-\varsigma}} \leq \mathrm{e}^{L^{1-\varsigma}} .
$$


Given $x, y \in \Lambda=\Lambda_{L}$ with $\|x-y\| \geq L / 100$, we proceed as in the derivation of (3.45) (with $\Theta=\emptyset$ ) to obtain, using (3.58),

$$
\left\|\chi_{y} R_{\omega, \Lambda}\left(E^{\prime}\right) \chi_{x}\right\| \leq \mathrm{e}^{\left.-m_{3}(\|x-y\|-\ell)\right)} 4 L^{2 d} e^{\ell^{1-\varsigma}} \leq \mathrm{e}^{-M_{1}\|x-y\|},
$$

where $M_{1}$ is as in (3.56).

Lemma 3.17. Suppose the scale $\ell$ is $(E, m, \varsigma, p)$-good, where $E \in \mathbb{R}, m>0,0<$ $\varsigma<1$, and $p>0$. Then, if $L=\ell^{1+\eta}$, where $0<\eta<p$, we have

$$
\mathbb{P}\left\{\Lambda_{L}(x) \text { is }(\boldsymbol{\omega}, E, m, \varsigma, \eta) \text {-pgood }\right\} \geq 1-2^{d} L^{-\frac{p-\eta}{1+\eta} d} \quad \text { for all } x \in \mathbb{R}^{d} .
$$

Proof. It follows from (3.7) and (3.52) that

$$
\mathbb{P}\left\{\Lambda_{L} \text { is not } E \text {-pgood }\right\}<\left\{2 L^{\frac{\eta}{1+\eta}}\right\}^{d} L^{-\frac{p d}{1+\eta}}=2^{d} L^{-\frac{p-\eta}{1+\eta} d} .
$$

3.3.2. Suitable coverings of annuli. Given scales $L_{1}<L_{2}$, we consider the open annulus

$$
\Lambda_{L_{2}, L_{1}}(x):=\Lambda_{L_{2}}(x) \backslash \bar{\Lambda}_{L_{1}}(x)=\left\{y \in \mathbb{R}^{d} ; L_{1} / 2<\|y-x\|<L_{2} / 2\right\} .
$$

We let $\bar{\Lambda}_{L_{2}, L_{1}}(x):=\overline{\Lambda_{L_{2}, L_{1}}(x)}$ be the closed annulus, and set $\Lambda_{\infty, L}(x):=\mathbb{R}^{d} \backslash \bar{\Lambda}_{L}(x)$.

Definition 3.18. Given scales $\ell, L_{1}, L_{2}$ with $L_{1}<L_{2}$ and $\ell<\left(L_{1}-L_{2}\right) / 2$, a suitable $\ell$-covering of an annulus $\Lambda_{L_{2}, L_{1}}(x)$ is a collection of boxes $\Lambda_{\ell}$ of the form

$$
\mathcal{G}_{\Lambda_{L_{2}, L_{1}}(x)}^{(\ell)}=\left\{\Lambda_{\ell}(r)\right\}_{r \in \mathbb{G}_{\Lambda_{L_{2}, L_{1}}^{(x)}}^{(\ell)}},
$$

where

$$
\begin{aligned}
\mathbb{G}_{\Lambda_{L_{2}, L_{1}}(x)}^{(\ell)} & :=\left\{r \in x+\mathbb{U}_{L_{1}, \ell}+\alpha \ell \mathbb{Z}^{d} ; \Lambda_{\ell}(r) \subset \Lambda_{L_{2}, L_{1}}(x)\right\}, \quad \text { with } \\
\mathbb{U}_{L_{1}, \ell} & :=\left\{0, L_{1} / 2,-L_{1} / 2,\left(L_{1}+\ell\right) / 2,-\left(L_{1}+\ell\right) / 2\right\}^{d} \backslash\left\{0, L_{1} / 2,-L_{1} / 2\right\}^{d}, \\
\alpha & \in[3 / 5,4 / 5] \cap\left\{\frac{L_{2}-L_{1}-2 \ell}{2 \ell n} ; n \in \mathbb{N}\right\} .
\end{aligned}
$$

Lemma 3.19. Consider scales $\ell, L_{1}, L_{2}$ with $L_{1}<L_{2}$ and $\ell<\left(L_{2}-L_{1}\right) / 7$. Then every annulus $\Lambda_{L_{2}, L_{1}}(x)$ has a suitable $\ell$-covering, and for any suitable $\ell$-covering $\mathcal{G}_{\Lambda_{L_{2}, L_{1}}(\ell)}^{(\ell)}$ of $\Lambda_{L_{2}, L_{1}}(x)$ we have

$$
\Lambda_{L_{2}, L_{1}}(x)=\bigcup_{r \in \mathbb{G}_{\Lambda_{L_{2}, L_{1}}^{(x)}}^{(x)}} \Lambda_{\ell}(r),
$$

given $y \in \Lambda_{L_{2}, L_{1}}(x)$ there is $r \in \mathbb{G}_{\Lambda_{L_{2}, L_{1}}(x)}^{(\ell)}$ with $\Lambda_{\ell / 5}(y) \cap \Lambda_{L_{2}, L_{1}}(x) \subset \Lambda_{\ell}(r)$,

$\# \mathbb{G}_{\Lambda_{L_{2}, L_{1}}(x)}^{(\ell)} \leq\left(2 L_{2} / \ell\right)^{d} \# \mathbb{U}_{L_{1}, \ell} \leq\left(10 L_{2} / \ell\right)^{d}$. 
Definition 3.18 is similar to Definition 3.12, and Lemma 3.19 is proven similarly to Lemma 3.13, but there are some differences. In particular, we do not have the analog of (3.51).

As in Definition 3.14, the standard $\ell$-covering of $\Lambda_{L_{2}, L_{1}}(x)$ corresponds to

$$
\alpha=\alpha_{L_{2}, L_{1}, \ell}:=\max \left\{[3 / 5,4 / 5] \cap\left\{\frac{L_{2}-L_{1}-\ell}{2 \ell n} ; n \in \mathbb{N}\right\}\right\} .
$$

\section{The multiscale analysis with a Wegner estimate}

We will prove the following theorem.

Theorem 4.1. Let $H_{\omega}$ be a generalized Anderson Hamiltonian on $\mathrm{L}^{2}\left(\mathbb{R}^{d}\right)$. Fix $p \in$ ]1/3,3/8[ and $\left.\varsigma, \varsigma^{\prime} \in\right] 0,1\left[\right.$. Then there exist an energy $E_{0}>0$, a rate of decay $m>0$, and a scale $L_{0}$, all depending only on $d, V_{\mathrm{per}}, \delta_{ \pm}, u_{ \pm}, U_{+}, \mu, p, \varsigma, \varsigma^{\prime}$, such that all scales $L \geq L_{0}$ are $\left(E, m, \varsigma, \varsigma^{\prime}, p\right)$-extra good for all energies $E \in\left[0, E_{0}\right]$. In particular, all scales $L \geq L_{0}$ are $(E, m, \varsigma, p)$-good for all energies $E \in\left[0, E_{0}\right]$.

To prove the theorem we first obtain an a priori estimate on the probability that a box $\Lambda_{L}$ is good with an adequate supply of free sites for all energies in an interval at the bottom of the spectrum (Proposition 4.3). Next, we perform a multiscale analysis to show that if such a probabilistic estimate holds for a given energy at a sufficiently large scale, then it holds all large scales (Proposition 4.6). Theorem 4.1 is an immediate consequence of Propositions 4.3 and 4.6.

Remark 4.2. If 0 is not an atom for the measure $\mu$ in (2.6), Proposition 4.5 provides an alternative to Proposition 4.3, giving an a priori estimate in a fixed interval at the bottom of the spectrum for sufficiently high disorder. If we also have $\mu([0, t]) \leq C t^{\gamma}$, with $\gamma>0$ appropriately large, Propositions 4.5 and 4.6 (and their proofs) yield an alternative high disorder version of Theorem 4.1.

\subsection{A priori finite volume estimates}

We set $\tilde{q}=\max \{q, 2\}$, where $q \in \mathbb{N}$ is the period of the background periodic operator $V_{\text {per }}$ in (2.10).

Proposition 4.3. Let $H_{\omega}$ be a generalized Anderson Hamiltonian on $\mathrm{L}^{2}\left(\mathbb{R}^{d}\right)$, and fix $p>0$ and $0<\varepsilon \leq 1$. There exists $\tilde{L}=\tilde{L}\left(d, V_{\text {per }}, u_{-}, \delta_{-}, \mu, p, \varepsilon\right)$ such that for all scales $L \geq \tilde{L}$ and all $x \in \mathbb{R}^{d}$ we have

$\mathbb{P}\left\{H_{\boldsymbol{\omega}, t_{S}, \Lambda_{L}(x)} \geq\left((p+1) d \log \left(L+\delta_{+}+\tilde{q}\right)\right)^{-(2+\varepsilon) / d}\right.$ for all $\left.\boldsymbol{t}_{S} \in[0,1]^{S}\right\} \geq 1-L^{-p d}$,

where $S=S_{x, L, q}=\widetilde{\Lambda}_{L}(x) \backslash \tilde{q} \mathbb{Z}^{d}$. In particular, setting

$$
E_{L}=\frac{1}{2}\left((p+1) d \log \left(L+\delta_{+}+\tilde{q}\right)\right)^{-(2+\varepsilon) / d} \quad \text { and } \quad m_{L}=\frac{1}{2} \sqrt{E_{L}}
$$


it follows that for all scales $L \geq \tilde{L}, x \in \mathbb{R}^{d}, t_{S} \in[0,1]^{S}$, and energies $E \in\left[0, E_{L}\right]$, we have, with probability $\geq 1-L^{-p d}$,

$$
\left\|R_{\omega, t_{S}, \Lambda_{L}(x)}(E)\right\| \leq 1 / E_{L},
$$

and, for all $y, y^{\prime} \in \Lambda_{L}$ with $\left\|y-y^{\prime}\right\| \geq 20 \sqrt{d}$,

$$
\left\|\chi_{y} R_{\omega, t_{S}, \Lambda_{L}(x)}(E) \chi_{y^{\prime}}\right\| \leq\left(2 / E_{L}\right) \mathrm{e}^{-\frac{2}{3} \sqrt{E_{L}}\left\|y-y^{\prime}\right\|} .
$$

In particular, given $\left.\varsigma, \varsigma^{\prime} \in\right] 0,1\left[\right.$, there is $\tilde{\tilde{L}}=\tilde{\tilde{L}}\left(d, V_{\mathrm{per}}, u_{-}, \delta_{-}, \mu, p, \varsigma, \varsigma^{\prime}, \varepsilon\right)$ such that all scales $L \geq \tilde{\tilde{L}}$ are $\left(E, m_{L}, \varsigma, \varsigma^{\prime}, p\right)$-extra good for all energies $E \in\left[0, E_{L}\right]$.

Proof. It suffices to prove (4.1), since given $H_{\omega, t_{S}, \Lambda_{L}(x)} \geq 2 E_{L}$, for all $E \in\left[0, E_{L}\right]$ we get immediately (4.3), and (4.4) follows by the Combes-Thomas estimate. (We use the precise estimate given in [GK2, Eq. (19)], which is also valid for finite volume operators with Dirichlet boundary condition.) Moreover, in view of (2.12) and (2.14), it suffices to prove (4.1) for the case when $U=0$, and $u_{\zeta}=u_{-} \chi_{\Lambda_{\delta_{-}}(\zeta)}$ for all $\zeta \in \tilde{q} \mathbb{Z}^{d}, u_{\zeta}=0$ otherwise.

So let

$$
H_{\omega}^{(q)}=H_{0}+V_{\omega}^{(q)} \quad \text { with } \quad V_{\omega}^{(q)}(x):=\sum_{\zeta \in \tilde{q} \mathbb{Z}^{d}} \omega_{\zeta} u(x-\zeta),
$$

where $u=u_{-} \chi_{\Lambda_{\delta_{-}}(0)}$. Note that $H_{\omega}^{(q)}$ is an Anderson Hamiltonian as in Definition 2.1, except that $\mathbb{Z}^{d}$ was replaced by $\tilde{q} \mathbb{Z}^{d}$ and the periodic potential has period $\tilde{q}$, and hence its integrated density of states $N^{(q)}(E)$ is well defined with the usual properties (cf. [CL, PF]). Given a box $\Lambda$, we define the corresponding finite volume operator $H_{\omega, \Lambda}^{(q)}$ as in (2.16). For scales $L \in \tilde{q} \mathbb{N}$ we set

$$
N_{\omega, \Lambda_{L}}^{(q)}(E):=\operatorname{tr} \chi_{]-\infty, E]}\left(\widetilde{H}_{\omega, \Lambda_{L}}^{(q)}\right),
$$

where

$$
\widetilde{H}_{\omega, \Lambda}^{(q)}:=H_{0, \Lambda}+\widetilde{V}_{\omega, \Lambda}^{(q)} \quad \text { on } \mathrm{L}^{2}(\Lambda),
$$

where $H_{0, \Lambda}$ is as in (2.17) and $\widetilde{V}_{\omega, \Lambda}^{(q)}$ is the restriction of $V_{\omega}^{(q)}$ to $\Lambda$. In general $\widetilde{V}_{\omega, \Lambda}^{(q)} \neq$ $V_{\omega, \Lambda}^{(q)}$, but we have (2.20).

We recall (e.g., [CL, Eq. (VI.15) on page 311]) that

$$
\mathbb{E}\left(N_{\omega, \Lambda_{L}}^{(q)}(E)\right) \leq N^{(q)}(E)\left|\Lambda_{L}\right| \quad \text { for all } L \in \tilde{q} \mathbb{N} .
$$

We now use the Lifshitz tails estimate as in [Klo3, Remark 7.1] (note that it applies with $\mu$ as in (2.6)):

$$
\lim _{E \downarrow 0} \frac{\log \left|\log N^{(q)}(E)\right|}{\log E} \leq-\frac{d}{2} .
$$


It follows that there is an energy $E_{1}=E_{1}\left(d, V_{\text {per }}, u_{-}, \delta_{-}, \mu, \varepsilon\right)>0$ such that

$$
N^{(q)}(E) \leq \mathrm{e}^{-E^{-d /(2+\varepsilon)}} \quad \text { for all energies } E \leq E_{1} .
$$

Combining (4.6)-(4.10), and using Chebyshev's inequality, we find that for all scales $L \in \tilde{q} \mathbb{N}, x \in \mathbb{R}^{d}$, and energies $E \leq E_{1}$,

$$
\mathbb{P}\left\{\sigma\left(\widetilde{H}_{\omega, \Lambda_{L}(x)}^{(q)}\right) \cap[0, E] \neq \emptyset\right\} \leq \mathbb{E}\left(N_{\omega, \Lambda_{L}(x)}^{(q)}(E)\right) \leq \mathrm{e}^{-E^{-d /(2+\varepsilon)}} L^{d},
$$

and hence

$$
\mathbb{P}\left\{\tilde{H}_{\omega, \Lambda_{L}(x)}^{(q)} \geq \min \left\{((p+1) d \log L)^{-(2+\varepsilon) / d}, E_{1}\right\}\right\} \geq 1-L^{-p d} .
$$

To get (4.1) from (4.12), given a scale $L \geq 1$ we set

$$
L_{q}:=\min \left\{L^{\prime} \in \tilde{q} \mathbb{N} ; L+\delta_{+} \leq L^{\prime}\right\}
$$

It follows from (2.20) that

$$
\chi_{\Lambda_{L}(x)} \widetilde{V}_{\omega, \Lambda_{L_{q}}(x)}^{(q)}=V_{\omega, \Lambda_{L}(x)}^{(q)}
$$

Since we are using a Dirichlet boundary condition for the Laplacian, we conclude that $\inf \sigma\left(H_{\omega, \Lambda_{L}(x)}^{(q)}\right) \geq \inf \sigma\left(\widetilde{H}_{\omega, \Lambda_{L_{q}}(x)}^{(q)}\right)$. Since $L+\delta_{+} \leq L_{q}<L+\delta_{+}+\tilde{q}$, we conclude that

$$
\mathbb{P}\left\{H_{\omega, \Lambda_{L}(x)}^{(q)} \geq \min \left\{\left((p+1) d \log \left(L+\delta_{+}+\tilde{q}\right)\right)^{-(2+\varepsilon) / d}, E_{1}\right\}\right\} \geq 1-L^{-p d}
$$

for all $L \geq 1$. The desired estimate (4.1) follows for all scales $L \geq \tilde{L}$, where $\tilde{L}=$ $\tilde{L}\left(d, V_{\text {per }}, u_{-}, \delta_{-}, \mu, p, \varepsilon\right)$.

Remark 4.4. In the absence of a periodic background potential, i.e., $V_{\text {per }}=0$, one can prove a slightly modified form of Proposition 4.3 using ideas from [BoK] instead of Lifshitz tails. As in the proof of Proposition 4.3, it suffices to consider the operator $H_{\omega}=-\Delta+V_{\omega}$, where $V_{\omega}$ is as in (4.5). Setting $K>10 \delta_{-}, \Lambda=\Lambda_{L}$, It follows from the lower bound in (2.14) that there exists a constant $c_{u_{-}, \delta_{-}, d}>0$ such that

$$
\bar{V}_{\omega_{\Lambda}}(x):=\frac{1}{K^{d}} \int_{\Lambda_{K}(0)} V_{\omega_{\Lambda}}(x-a) \mathrm{d} a \geq c_{u_{-}, \delta_{-}, d} Y_{\omega, \Lambda} \chi_{\Lambda}(x),
$$

where

$$
Y_{\boldsymbol{\omega}, \Lambda}:=\min _{\xi \in \widetilde{\Lambda}} \frac{1}{K^{d}} \sum_{\zeta \in \Lambda_{K / 3}(\xi)} \omega_{\zeta} .
$$

It follows from standard estimates (e.g., [Y, Proposition 3.3.1]) that, with $\bar{\mu}$ and $\sigma$ the mean and standard deviation of the probability measure $\mu$, we have

$$
\mathbb{P}\left\{\frac{1}{K^{d}} \sum_{\zeta \in \Lambda_{K / 3}(\xi)} \omega_{\zeta} \leq \frac{\bar{\mu}}{2}\right\} \leq \mathrm{e}^{-A K^{d}},
$$


where

$$
A=A_{\mu, d}=\frac{\bar{\mu}}{3^{d} 8 \sigma^{2}(1+\bar{\mu} / 2)}>0
$$

and hence

$$
\mathbb{P}\left\{Y_{\boldsymbol{\omega}, \Lambda} \leq \bar{\mu} / 2\right\} \leq L^{d} \mathrm{e}^{-A K^{d}} .
$$

It follows from (4.16) and (4.20) that with $c_{u_{-}, \delta_{-}, d}^{\prime}=\frac{1}{2} c_{u_{-}, \delta_{-}, d}$,

$$
\mathbb{P}\left\{\bar{V}_{\omega_{\Lambda}}>c_{u_{-}, \delta_{-}, d}^{\prime} \bar{\mu} \chi_{\Lambda}\right\} \geq 1-L^{d} \mathrm{e}^{-A K^{d}},
$$

so, if $\bar{V}_{\omega_{\Lambda}}>c_{u_{-}, \delta_{-}, d}^{\prime} \bar{\mu} \chi_{\Lambda}$, we have

$$
\bar{H}_{\omega, \Lambda}:=-\Delta_{\Lambda}+\chi_{\Lambda} \bar{V}_{\omega_{\Lambda}} \geq c_{u_{-}, \delta_{-}, d}^{\prime} \bar{\mu} \quad \text { on } \mathrm{L}^{2}(\Lambda) .
$$

Thus, if $\varphi \in C_{c}^{\infty}(\Lambda)$ with $\|\varphi\|=1$, we have

$$
\begin{aligned}
\left\langle\varphi, H_{\omega, \Lambda} \varphi\right\rangle_{\Lambda} & =\left\langle\varphi, \bar{H}_{\omega, \Lambda} \varphi\right\rangle_{\Lambda}+\left\langle\varphi,\left(V_{\omega_{\Lambda}}-\bar{V}_{\omega_{\Lambda}}\right) \varphi\right\rangle_{\Lambda} \\
& \geq c_{u_{-}, \delta_{-}, d}^{\prime} \bar{\mu}+\left\langle\varphi,\left(V_{\omega_{\Lambda}}-\bar{V}_{\omega_{\Lambda}}\right) \varphi\right\rangle_{\mathbb{R}^{d}} \\
& \geq c_{u_{-}, \delta_{-}, d}^{\prime} \bar{\mu}+\left\langle\varphi, V_{\omega_{\Lambda}} \varphi\right\rangle_{\mathbb{R}^{d}}-\frac{1}{K^{d}} \int_{\Lambda_{K}(0)}\left\langle\varphi(\cdot+a), V_{\omega_{\Lambda}} \varphi(\cdot+a)\right\rangle \mathrm{d} a \\
& \geq c_{u_{-}, \delta_{-}, d}^{\prime} \bar{\mu}-\frac{1}{K^{d}} \int_{\Lambda_{K}(0)}\left|\left\langle\varphi, V_{\omega_{\Lambda}} \varphi\right\rangle-\left\langle\varphi(\cdot+a), V_{\omega_{\Lambda}} \varphi(\cdot+a)\right\rangle\right| \mathrm{d} a \\
& \geq c_{u_{-}, \delta_{-}, d}^{\prime} \bar{\mu}-c_{u}^{\prime} K\left\|\nabla_{\Lambda} \varphi\right\|_{\Lambda} \geq c_{u_{-}, \delta_{-}, d}^{\prime} \bar{\mu}-c_{u}^{\prime} K\left\langle\varphi, H_{\omega, \Lambda} \varphi\right\rangle_{\Lambda}^{1 / 2},
\end{aligned}
$$

where we used

$$
\|\varphi(\cdot+a)-\varphi\|_{\mathbb{R}^{d}}=\left\|\left(\mathrm{e}^{a \cdot \nabla}-1\right) \varphi\right\|_{\mathbb{R}^{d}} \leq|a|\|\nabla \varphi\|_{\mathbb{R}^{d}}=|a|\left\|\nabla_{\Lambda} \varphi\right\|_{\Lambda} .
$$

It follows that there is $\tilde{K}_{u, d}>0$ such that for $K>\tilde{K}_{u, d}$ we have

$$
\left\langle\varphi, H_{\omega, \Lambda} \varphi\right\rangle_{\Lambda} \geq c_{u_{-}, \delta_{-}, d}^{\prime \prime} \frac{\bar{\mu}^{2}}{K^{2}} .
$$

Since this holds for all $\varphi \in C_{c}^{\infty}(\Lambda)$ with $\|\varphi\|=1$, we have

$$
H_{\omega, \Lambda} \geq c_{u_{-}, \delta_{-}, d}^{\prime \prime} \bar{\mu}^{2} / K^{2} \quad \text { on } \mathrm{L}^{2}(\Lambda) .
$$

From (4.21) and (4.26) we get

$$
\mathbb{P}\left\{H_{\boldsymbol{\omega}, \Lambda} \geq c_{u_{-}, \delta_{-}, d}^{\prime \prime} \bar{\mu}^{2} / K^{2}\right\}>1-L^{d} \mathrm{e}^{-A K^{d}} .
$$

Given $p>0$, we take $K=\left(\frac{(p+1) d}{A_{\mu, d}} \log L\right)^{1 / d}$ to get

$$
\mathbb{P}\left\{H_{\omega, \Lambda_{L}} \geq 2 C_{u_{-}, \delta_{-}, \mu, d, p}(\log L)^{-2 / d}\right\}>1-L^{-p d}
$$

for $L \geq \tilde{L}_{u_{-}, \delta_{-}, \mu, d, p}$, where $C_{u_{-}, \delta_{-}, \mu, d, p}>0$ is an appropriate constant. 
We then take $n \in \mathbb{N}$ and let $S=S_{\Lambda}=n \mathbb{Z}^{d} \cap \Lambda$. If $n \ll K$, we find, as in (4.16), that for all $t_{S} \in[0,1]^{S}$ we have

$$
\bar{V}_{\omega_{\Lambda}, t_{S}}(x):=\frac{1}{K^{d}} \int_{\Lambda_{K}(0)} V_{\omega_{\Lambda}, t_{S}}(x-a) \mathrm{d} a \geq c_{u_{-}, \delta_{-}, d} Y_{\omega, S, \Lambda} \chi_{\Lambda}(x),
$$

where

$$
Y_{\boldsymbol{\omega}, S, \Lambda}:=\min _{\xi \in \widetilde{\Lambda}} \frac{1}{K^{d}} \sum_{\zeta \in \Lambda_{K / 3}(\xi) \backslash S} \omega_{\zeta} .
$$

Proceeding as above, we conclude that

$$
\mathbb{P}\left\{H_{\omega, t_{S}, \Lambda_{L}} \geq 2 C_{u_{-}, \delta_{-}, \mu, d, p, q}(\log L)^{-2 / d} \text { for all } t_{S} \in[0,1]^{S}\right\}>1-L^{-p d}
$$

for $L \geq \tilde{L}_{u_{-}, \delta_{-}, \mu, d, p, q}$, where $C_{u_{-}, \delta_{-}, \mu, d, p, q}>0$ is an appropriate constant.

If 0 is not an atom for the measure $\mu$ in (2.6), i.e., if $\mu(\{0\})=0$, we can also obtain a high disorder a priori finite volume estimate.

Proposition 4.5. Consider the generalized Anderson Hamiltonian $H_{\omega, \lambda}=H_{0}+\lambda V_{\omega}$ on $\mathrm{L}^{2}\left(\mathbb{R}^{d}\right)$, where $H_{0}$ and $V_{\omega}$ are as in $(2.10)$ and $\lambda>0$. Suppose 0 is not an atom for the measure $\mu$ in (2.6). There exists an energy $\widetilde{E}=\widetilde{E}\left(d, V_{\mathrm{per}}, u_{-}, \delta_{-}\right)>0$, such that, fixing $\left.E_{0} \in\right] 0, \widetilde{E}\left[\right.$ and $p>0$, given $L \geq 100\left(\delta_{+}+1\right)$ there exists a constant $\tilde{\lambda}(L)=\tilde{\lambda}\left(d, V_{\mathrm{per}}, u_{-}, \delta_{-}, \mu, p, E_{0}, L\right)$, non-decreasing as a function of $E_{0}$, such that for all $\lambda \geq \tilde{\lambda}(L)$ we have

$$
\mathbb{P}\left\{H_{\omega, t_{S}, \lambda, \Lambda_{L}(x)} \geq E_{0} \text { for all } \boldsymbol{t}_{S} \in[0,1]^{S}\right\} \geq 1-L^{-p d} \quad \text { for all } x \in \mathbb{R}^{d},
$$

where $S=S_{x, L, q}=\widetilde{\Lambda}_{L}(x) \backslash \tilde{q} \mathbb{Z}^{d}$. Thus, for all $E \in\left[0, E_{0}\left[, x \in \mathbb{R}^{d}, \boldsymbol{t}_{S} \in[0,1]^{S}\right.\right.$, and $\lambda \geq \tilde{\lambda}(L)$, it follows, with probability $\geq 1-L^{-p d}$, that

$$
\left\|R_{\omega, t_{S}, \lambda, \Lambda_{L}(x)}(E)\right\| \leq\left(E_{0}-E\right)^{-1},
$$

and, for $y, y^{\prime} \in \Lambda_{L},\left\|y-y^{\prime}\right\| \geq 20 \sqrt{d}$,

$$
\left\|\chi_{y} R_{\omega, t_{S}, \lambda, \Lambda_{L}(x)}(E) \chi_{y^{\prime}}\right\| \leq 2\left(E_{0}-E\right)^{-1} \mathrm{e}^{-\frac{2}{3} \sqrt{E_{0}-E}\left\|y-y^{\prime}\right\|} .
$$

In particular, given $\left.\varsigma, \varsigma^{\prime} \in\right] 0,1\left[\right.$ and $0<E_{1}<E_{0}<\widetilde{E}$, there is $\tilde{\tilde{L}}=\tilde{\tilde{L}}\left(d, \varsigma, \varsigma^{\prime}, E_{0}-E_{1}\right)$ such that for all energies $E \in\left[0, E_{1}\right]$ a scale $L \geq \tilde{\tilde{L}}$ is $\left(E, \frac{1}{2} \sqrt{E_{0}-E_{1}}, \varsigma, \varsigma^{\prime}, p\right)$-extra good if $\lambda \geq \tilde{\lambda}(L)$.

Proof. Similarly to the proof of Theorem 4.3, in view of (2.14) it suffices to consider the case when $u_{\zeta}=u_{-} \chi_{\Lambda_{\delta_{-}}(\zeta)}$ for all $\zeta \in \mathbb{Z}^{d}$. Given $t \geq 0$, we set $H(t)=H_{0}+$ $V(t)$, where $V(t)=t \sum_{\zeta \in \mathbb{Z}^{d}} u_{\zeta}$ is a periodic potential with period one. Then $E(t)=$ $\inf \sigma(H(t))$ is a strictly increasing continuous function of $t \geq 0$ with $E(0)=0$ (see [Klo2, Lemma 3.1 and its proof]); we set $E(\infty)=\lim _{t \rightarrow \infty} E(t)>0$. Given a box $\Lambda=\Lambda_{L}(x)$ we let $H_{\omega, \lambda, \Lambda}$ and $H_{\Lambda}(t)$ be the corresponding finite volume operators to 
$H_{\omega, \lambda}$ and $H(t) .\left(H_{\Lambda}(t)=H_{\omega, t, \Lambda}\right.$ with $\omega_{j}=1$ for all $j \in \mathbb{Z}^{d}$.) Since we are using a Dirichlet boundary condition, we have $H_{\Lambda}(t) \geq E(t)$, and hence

$$
H_{\omega, \lambda, \Lambda} \geq E(t) \quad \text { if } \lambda \omega_{j} \geq t \text { for all } j \in \widetilde{\Lambda}
$$

Given $\left.E_{0} \in\right] 0, E(\infty)\left[\right.$, let $t_{0}>0$ be defined by $E_{0}=E\left(t_{0}\right)$. We conclude that

$$
\mathbb{P}\left\{H_{\omega, \lambda, \Lambda} \geq E_{0}\right\} \geq 1-L^{d} \mu\left(\left[0, t_{0} / \lambda[)\right) .\right.
$$

Since $\mu(\{0\})=0$ by hypothesis, we have $\lim _{\lambda \rightarrow \infty} \mu\left(\left[0, t_{0} / \lambda[)\right)=0\right.$, and hence there exists $\tilde{\lambda}(L)=\tilde{\lambda}\left(d, V_{\text {per }}, u_{-}, \delta_{-}, \mu, p, E_{0}, L\right)<\infty$ such that

$$
\mathbb{P}\left\{H_{\omega, \lambda, \Lambda} \geq E_{0}\right\} \geq 1-L^{-p d} \quad \text { for } \lambda \geq \tilde{\lambda}(L) .
$$

To prove a similar estimate with free sites, we set $H_{\omega, \lambda}^{(q)}=H_{0}+\lambda V_{\omega}^{(q)}$ with $V_{\omega}^{(q)}$ as in (4.5). Proceeding as above, let $H^{(q)}(t)=H_{0}+V^{(q)}(t)$, where $V^{(q)}(t)=t \sum_{\zeta \in \tilde{q} \mathbb{Z}^{d}} u(x-\zeta)$, set $E^{(q)}(t)=\inf \sigma\left(H^{(q)}(t)\right)$, and let $E^{(q)}(\infty)=$ $\lim _{t \rightarrow \infty} E^{(q)}(t)>0$. Given $\left.E_{0} \in\right] 0, E^{(q)}(\infty)$ [, let $t_{0}^{(q)}>0$ be defined by $E_{0}=$ $E^{(q)}\left(t_{0}^{(q)}\right)$. Given a box $\Lambda=\Lambda_{L}$, we set $S=\widetilde{\Lambda}_{L} \backslash \tilde{q} \mathbb{Z}^{d}$. We conclude that there is $\tilde{\lambda}(L)<\infty$ such that for all $\lambda \geq \tilde{\lambda}(L)$ we have

$$
\mathbb{P}\left\{H_{\boldsymbol{\omega}, \boldsymbol{t}_{S}, \lambda, \Lambda} \geq E_{0} \text { for all } \boldsymbol{t}_{S} \in[0,1]^{S}\right\} \geq 1-(L / q)^{d} \mu\left(\left[0, t_{0}^{(q)} / \lambda\right)\right) \geq 1-L^{-p d},
$$

which is (4.32). As in Proposition 4.3, if $\lambda \geq \tilde{\lambda}(L)$, then for all $E \in\left[0, E_{0}\right.$ [ and $\boldsymbol{t}_{S} \in$ $[0,1]^{S}$, it follows, with probability $\geq 1-L^{-p d}$, that we have (4.33) and (4.34).

\subsection{The multiscale analysis}

We now state our single energy multiscale analysis for generalized Anderson Hamiltonians.

Proposition 4.6. Let $H_{\omega}$ be a generalized Anderson Hamiltonian on $\mathrm{L}^{2}\left(\mathbb{R}^{d}\right)$. Fix $E_{0}>0$, $p \in] 1 / 3,3 / 8\left[\right.$, and $\left.\varsigma, \varsigma^{\prime}, \tau, \rho_{1}, \rho_{2} \in\right] 0,1\left[\right.$ with $\tau<\varsigma$ and $\rho_{2}=\rho_{1}^{n_{1}}, n_{1} \in \mathbb{N}$, such that

$$
1 /(1+p)<\rho_{1}<\frac{3}{4}(1-\varsigma) \text { and } p<\frac{1}{2} \rho_{1}\left(1-\varsigma^{\prime}\right)-\rho_{2} .
$$

There exists a finite scale $\widetilde{L}_{0}=\widetilde{L}_{0}\left(d, V_{\mathrm{per}}, \delta_{ \pm}, u_{ \pm}, U_{+}, \mu, E_{0}, p, \rho_{1}, \rho_{2}, \varsigma, \varsigma^{\prime}, \tau\right)$ with the following property: given an energy $E \in\left[0, E_{0}\right]$, a scale $L_{0} \geq \widetilde{L}_{0}$, and a number

$$
m_{0} \geq L_{0}^{-\tau}
$$

if all scales $L \in\left[L_{0}, L_{0}^{1 / \rho_{1} \rho_{2}}\right]$ are $\left(E, m_{0}, \varsigma, \varsigma^{\prime}, p\right)$-extra good, it follows that every scale $L \geq L_{0}$ is $\left(E, m_{0} / 2, \varsigma, \varsigma^{\prime}, p\right)$-extra good.

Remark 4.7. To satisfy (4.39) and (4.40), we may pick $p=\frac{3}{8}-$, and appropriate $\rho_{1}=$ $\frac{3}{4}-, \varsigma=0+, \varsigma^{\prime}=0+, \tau=0+, \rho_{2}=0+$. 
Remark 4.8. The restriction $p \in] 1 / 3,3 / 8[$ comes from the use of the quantitative unique continuation principle, stated in Theorem A.1 and used in the form given in Corollary A.2(i), which gives a lower bound of the form $R^{-C R^{4 / 3}}$ in (A.6). It is instructive to see what happens if this lower bound was of the form $R^{-C R^{\gamma}}$ for some $\gamma>0$. In the multiscale analysis, Lemma 4.11 requires $1 /(1+p)<\rho_{1}$ in (4.46). The lower bound of (A.6) is used to prove Lemma 4.14; the important estimate (4.59) is useful only if $\gamma \rho_{1}<1$. Lemma 4.16 uses $p<\frac{1}{2} \rho_{1}$ to get the probability estimate (4.113). We conclude that the multiscale analysis requires

$$
\begin{gathered}
\gamma<\frac{1+\sqrt{3}}{2} \quad \text { and } \quad \gamma-1<p<\frac{1}{2 \gamma}, \\
\frac{1}{1+p}<\rho_{1}<\frac{1}{\gamma}(1-\varsigma) \quad \text { and } \quad p<\frac{1}{2} \rho_{1}\left(1-\varsigma^{\prime}\right)-\rho_{2} .
\end{gathered}
$$

Since the quantitative unique continuation principle gives $\gamma=4 / 3<(1+\sqrt{3}) / 2$, we can perform the multiscale analysis with $p \in] 1 / 3,3 / 8[$ and (4.39).

The proof of Pproposition 4.6 will require several lemmas and definitions. We fix an energy $E \in\left[0, E_{0}\right]$, and let $p, \varsigma, \varsigma^{\prime}, \rho_{1}, \rho_{2}, n_{1}, \tau$ be as in Proposition 4.6, satisfying (4.39).

Definition 4.9. A collection $\mathcal{L}$ of scales is called $\left(E, \varsigma, \varsigma^{\prime}, p, \tau\right)$-extra good if for each $\ell \in \mathcal{L}$ there is a rate of decay $m_{\ell}$, with

$$
m_{\ell} \geq \ell^{-\tau}
$$

such that for each box $\Lambda_{\ell}$ there is a $\left(\Lambda_{\ell}, E, m_{\ell}, \varsigma, \varsigma^{\prime}\right)$-extra good event $\mathcal{E}_{\Lambda_{\ell}}$ satisfying (3.16).

In the following definitions and lemmas, given a scale $L$, we set $\ell_{1}=L^{\rho_{1}}$ and $\ell_{2}=$ $\ell_{1}^{\rho_{2}}=L^{\rho_{1} \rho_{2}}$. We also set $L_{n}=\ell_{1}^{\rho_{1}^{n}}$ for $n=0,1, \ldots, n_{1}$; note $L_{0}=\ell_{1}$ and $L_{n_{1}}=\ell_{2}$.

We start by defining an event that incorporates [BoK, property $(*)]$. Note that by writing " $\mathcal{R}=\left\{\Lambda_{\ell}(r)\right\}_{r \in R}$ is the standard $\ell$-covering of $\Lambda_{L}$ " (cf. Definitions 3.12 and 3.14), we will mean that $\mathcal{R}=\mathcal{G}_{\Lambda_{L}}^{(\ell)}$ as in (3.47) with $\alpha$ as in (3.55); in particular, $R=\mathbb{G}_{\Lambda_{L}}^{(\ell)}$ as in (3.48).

Definition 4.10. Given a box $\Lambda_{\ell_{1}}$, let $\mathcal{R}_{n}=\left\{\Lambda_{L_{n}}(r)\right\}_{r \in R_{n}}$ be the standard $L_{n}$-covering of $\Lambda_{\ell_{1}}$. Fix a number $K_{2} \in \mathbb{N}$. Then:

(i) A box $\Lambda_{\ell_{1}}$ is said to be $\left(\omega, E, K_{2}\right)$-notsobad if there is $\Theta=\bigcup_{r \in R_{n_{1}}^{\prime}} \Lambda_{3 \ell_{2}}(r)$, where $R_{n_{1}}^{\prime} \subset R_{n_{1}}$ with \# $R_{n_{1}}^{\prime} \leq K_{2}$, such that for all $x \in \Lambda_{\ell_{1}} \backslash \Theta$ there is an $\left(\omega, E, m_{L_{n}}, \varsigma\right)$ good box $\Lambda_{L_{n}}(r)$ with $r \in R_{n}$ for some $n \in\left\{1, \ldots, n_{1}\right\}$ and $\Lambda_{L_{n} / 5}(x) \cap \Lambda_{\ell_{1}} \subset$ $\Lambda_{L_{n}}(r)$.

(ii) An event $\mathcal{N}$ is $\left(\Lambda_{\ell_{1}}, E, K_{2}\right)$-notsobad if $\mathcal{N} \in \mathcal{F}_{\Lambda_{\ell_{1}}}$ and the box $\Lambda_{\ell_{1}}$ is $\left(\omega, E, K_{2}\right)$ notsobad for all $\omega \in \mathcal{N}$. 
Lemma 4.11. Suppose $\left\{L_{n} ; n=1, \ldots, n_{1}\right\}$ is $\left(E, \varsigma, \varsigma^{\prime}, p, \tau\right)$-extra good. There exists a constant $\widehat{K}_{2}=\widehat{K}_{2}\left(d, p, \rho_{1}, \rho_{2}\right)$, and for $K_{2} \in \mathbb{N}$ with $K_{2} \geq \widehat{K}_{2}$ a constant $\widehat{\ell}_{1}=$ $\widehat{\ell}_{1}\left(d, p, \rho_{1}, \rho_{2}, K_{2}\right)$, such that for any box $\Lambda_{\ell_{1}}$ with $\ell_{1} \geq \widehat{\ell}_{1}$ there exists a $\left(\Lambda_{\ell_{1}}, E, K_{2}\right)$ notsobad event $\mathcal{N}_{\Lambda_{\ell_{1}}}$ with

$$
\mathbb{P}\left\{\mathcal{N}_{\Lambda_{\ell_{1}}}\right\}>1-\ell_{1}^{-5 d}
$$

Proof. Given $\Lambda_{L_{n-1}}(r) \in \mathcal{R}_{n-1}$, we set

$$
\begin{aligned}
\mathcal{R}_{n}(r) & :=\left\{\Lambda_{L_{n}}(s) \in \mathcal{R}_{n} ; \Lambda_{L_{n}}(s) \cap \Lambda_{L_{n-1}}(r) \neq \emptyset\right\}, \\
R_{n}(r) & :=\left\{s \in R_{n} ; \Lambda_{L_{n}}(s) \in \mathcal{R}_{n}(r)\right\} .
\end{aligned}
$$

We have $\Lambda_{L_{n-1}}(r) \subset \bigcup_{s \in R_{n}(r)} \Lambda_{L_{n}}(s)$ and, similarly to (3.52), \#R $(r) \leq\left(3 L_{n-1} / L_{n}\right)^{d}$. Fix a number $K^{\prime}$, and define the event $\mathcal{N}_{\Lambda_{\ell_{1}}}$ as consisting of $\omega \in \Omega$ such that, for all $n=1, \ldots, n_{1}$ and all $r \in R_{n-1}$, we have $\omega \in \mathcal{E}_{\Lambda_{L_{n}}(s)}$ for all $s \in R_{n}(r)$, with the possible exception of at most $K^{\prime}$ disjoint boxes $\Lambda_{L_{n}}(s)$ with $s \in R_{n}(r)$. We clearly have $\mathcal{N}_{\Lambda_{\ell_{1}}} \in \mathcal{F}_{\Lambda_{\ell_{1}}}$. Since $\left\{L_{n} ; n=1, \ldots, n_{1}\right\}$ is $\left(E, \varsigma, \varsigma^{\prime}, p, \tau\right)$-extra good, the probability of its complementary event to $\mathcal{N}_{\Lambda_{\ell_{1}}}$ can be estimated from (3.16):

$$
\begin{aligned}
\mathbb{P}\left\{\Omega \backslash \mathcal{N}_{\Lambda_{\ell_{1}}}\right\} & \leq \sum_{n=1}^{n_{1}}\left(\frac{2 \ell_{1}}{L_{n-1}}\right)^{d}\left(\frac{3 L_{n-1}}{L_{n}}\right)^{K^{\prime} d} L_{n}^{-K^{\prime} p d} \\
& \leq 2^{d} 3^{K^{\prime} d} n_{1} \ell_{1}^{-\rho_{1}^{n_{1}-1}\left(K^{\prime}\left(\rho_{1}(p d+d)-d\right)+d\right)+d} \\
& =2^{d} 3^{K^{\prime} d} n_{1} \ell_{1}^{-d\left(\rho_{1}^{n_{1}-1}\left(K^{\prime}\left(\rho_{1}(p+1)-1\right)+1\right)-1\right)} \leq \ell_{1}^{-5 d},
\end{aligned}
$$

where the last inequality holds for all large $\ell_{1}$ after choosing $K^{\prime}$ sufficiently large using (4.39).

Given $\omega \in \mathcal{N}_{\Lambda_{\ell_{1}}}$, then for each $n=1, \ldots, n_{1}$ and $r \in R_{n-1}$ we can find $s_{1}, \ldots, s_{K^{\prime \prime}}$ $\in R_{n}(r)$ with $K^{\prime \prime} \leq K^{\prime}-1$ such that $\omega \in \mathcal{E}_{\Lambda_{L_{n}}(s)}$ if $s \in R_{n(r)}$ and $s \notin \bigcup_{j=1}^{K^{\prime \prime}} \Lambda_{3 L_{n}}\left(s_{j}\right)$. (Here we need boxes of side $3 L_{n}$ because we only ruled out the existence of $K^{\prime}$ disjoint boxes of side $L_{n}$.) Since each box $\Lambda_{3 L_{n}}\left(s_{j}\right)$ is contained in the union of at most $C^{\prime \prime}$ boxes in $\mathcal{R}_{n}$, we conclude that for each $\omega \in \mathcal{N}_{\Lambda_{\ell_{1}}}$ there are $t_{1}, \ldots, t_{K^{\prime \prime \prime}} \in R_{n_{1}}$ with $K^{\prime \prime \prime} \leq K_{2}=\left(C^{\prime \prime}\left(K^{\prime}-1\right)\right)^{n_{1}}$ such that, setting $\Theta=\bigcup_{t_{j}=1}^{K^{\prime \prime \prime}} \Lambda_{3 \ell_{2}}\left(t_{j}\right)$, for all $x \in \Lambda_{\ell_{1}} \backslash \Theta$ we have $\omega \in \mathcal{E}_{\Lambda_{L_{n}}(s)}$ for some $n=1, \ldots, n_{1}$ and $s \in R_{n}$ with $\Lambda_{L_{n} / 5}(x) \cap \Lambda_{\ell_{1}} \subset \Lambda_{L_{n}}(s)$.

Definition 4.12. Fix $K_{1}, K_{2} \in \mathbb{N}$. Then:

(i) An event $\mathcal{P}$ is called $\left(\Lambda, E, K_{1}, K_{2}\right)$-prepared if, with $\mathcal{R}=\left\{\Lambda_{\ell_{1}}(r)\right\}_{r \in R}$ being the standard $\ell_{1}$-covering of $\Lambda=\Lambda_{L}$, there exists a disjoint decomposition $R=R^{\prime} \sqcup R^{\prime \prime}$ with $\# R^{\prime \prime} \leq K_{1}$ such that

$$
\mathcal{P}=\left\{\bigcap_{r \in R^{\prime}} \mathcal{C}_{\Lambda_{\ell_{1}}(r)}\right\} \cap\left\{\bigcap_{r \in R^{\prime \prime}} \mathcal{N}_{\Lambda_{\ell_{1}}(r)}\right\},
$$




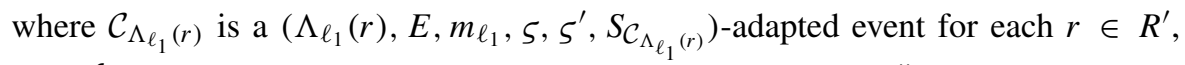
and $\mathcal{N}_{\Lambda_{\ell_{1}}(r)}$ is a $\left(\Lambda_{\ell_{1}}(r), E, K_{2}\right)$-notsobad event for each $r \in R^{\prime \prime}$. In this case we set

$$
\begin{aligned}
& S_{\mathcal{P}}:=\left\{s \in \tilde{\Lambda} ; s \in \Lambda_{\ell_{1}}(r) \Rightarrow r \in R^{\prime} \text { and } s \in S_{\mathcal{C}_{\Lambda_{\ell_{1}}(r)}}\right\} \\
& =\bigcup_{r \in R^{\prime}}\left(S_{\mathcal{C}_{\Lambda_{\ell_{1}}(r)}} \backslash \bigcup_{r^{\prime} \in R^{\prime} \backslash\{r\}}\left(\Lambda_{\ell_{1}}\left(r^{\prime}\right) \backslash S_{\left.\mathcal{C}_{\Lambda_{\ell_{1}}\left(r^{\prime}\right)}\right)}\right) \backslash \bigcup_{r \in R^{\prime \prime}} \Lambda_{\ell_{1}}(r) .\right.
\end{aligned}
$$

(ii) An event $\mathcal{Q}$ is called $\left(\Lambda, E, K_{1}, K_{2}\right)$-ready if it is the disjoint union of a finite number of $\left(\Lambda, E, K_{1}, K_{2}\right)$-prepared events, i.e., there exist disjoint $\left(\Lambda, E, K_{1}, K_{2}\right)$-prepared events $\left\{\mathcal{P}_{j}\right\}_{j=1, \ldots, J}$ such that

$$
\mathcal{Q}=\bigsqcup_{j=1}^{J} \mathcal{P}_{j}
$$

The set $S_{\mathcal{P}}$ in (4.48) is the maximal set with the required properties. It follows from (3.51) that

$$
\bigsqcup_{r \in R^{\prime}}\left\{S_{\mathcal{C}_{\Lambda_{\ell_{1}}(r)}} \cap \Lambda_{\ell_{1} / 5}(r)\right\} \subset S_{\mathcal{P}},
$$

and nothing would be lost if we had defined $S_{\mathcal{P}}$ by making (4.50) an equality.

Lemma 4.13. Suppose $\left\{L_{n} ; n=0,1, \ldots, n_{1}\right\}$ is $\left(E, \varsigma, \varsigma^{\prime}, p, \tau\right)$-extra good. For suffciently large $K_{1}, K_{2} \in \mathbb{N}$, depending only on $d, p, \rho_{1}, \rho_{2}$, if $L$ is taken large enough, depending only on $d, p, \rho_{1}, \rho_{2}, \varsigma^{\prime}, K_{1}, K_{2}$, the following holds:

(i) If $\mathcal{P}$ is a $\left(\Lambda, E, K_{1}, K_{2}\right)$-prepared event, then $S_{\mathcal{P}}$ is a $\varsigma^{\prime}$-abundant subset of $\widetilde{\Lambda}$ and $\mathcal{P} \in \mathcal{F}_{\Lambda \backslash S_{\mathcal{P}}}$.

(ii) There exists a $\left(\Lambda, E, K_{1}, K_{2}\right)$-ready event $\mathcal{Q}$ such that

$$
\mathbb{P}\{\mathcal{Q}\}>1-2 L^{-2 d} .
$$

Proof. Let $\mathcal{P}$ be a ( $\left.\Lambda, E, K_{1}, K_{2}\right)$-prepared event, as in (4.47), and let $S_{\mathcal{P}}$ be as in (4.48). In particular, $\mathcal{P} \in \mathcal{F}_{\Lambda \backslash S_{\mathcal{P}}}$. Since $\# R^{\prime \prime} \leq K_{1}$, it follows from (4.50), using (3.14), that for all boxes $\Lambda_{L / 5} \subset \Lambda$ we have, with $L$ sufficiently large,

$$
\#\left(S_{\mathcal{P}} \cap \Lambda_{L / 5}\right) \geq \ell_{1}^{\left(1-\varsigma^{\prime}\right) d}\left(\left(\frac{5}{4} \frac{L}{5 \ell_{1}}-2\right)^{d}-K_{1}\right) \geq L^{\left(1-\varsigma^{\prime}\right) d},
$$

and hence $S_{\mathcal{P}}$ is a $\varsigma^{\prime}$-abundant subset of $\widetilde{\Lambda}$.

We now use the hypothesis that $\left\{L_{n} ; n=0,1, \ldots, n_{1}\right\}$ is $\left(E, \varsigma, \varsigma^{\prime}, p, \tau\right)$-extra good. For each $r \in R$ we pick a $\left(\Lambda_{\ell_{1}}(r), E, m_{\ell_{1}}, \zeta, \varsigma^{\prime}\right)$-extra good event $\mathcal{E}_{\Lambda_{\ell_{1}}(r)}$ as in (3.15) with (3.16). Taking $K_{2}$ and $L$ sufficiently large so we can use Lemma 4.11, for each $r \in R$ we also pick a $\left(\Lambda_{\ell_{1}}(r), E, K_{2}\right)$-notsobad event $\mathcal{N}_{\Lambda_{\ell_{1}}(r)}$ with (4.44), and set $\mathcal{N}_{\Lambda_{\ell_{1}}(r)}^{*}=$ 
$\mathcal{N}_{\Lambda_{\ell_{1}}(r)} \backslash \mathcal{E}_{\Lambda_{\ell_{1}}(r)}$, clearly also a $\left(\Lambda_{\ell_{1}}(r), E, K_{2}\right)$-notsobad event. Given $K_{1} \in \mathbb{N}$, define the event $\mathcal{Q}$ by the disjoint union

$$
\begin{gathered}
\mathcal{Q}:=\bigsqcup_{\substack{R^{\prime} \subset R \\
\\
\left(R \backslash R^{\prime}\right) \leq K_{1}}} \mathcal{Q}\left(R^{\prime}\right), \quad \text { where } \\
\mathcal{Q}\left(R^{\prime}\right)=\left\{\bigcap_{r \in R^{\prime}} \mathcal{E}_{\Lambda_{\ell_{1}}(r)}\right\} \cap\left\{\bigcap_{r \in R \backslash R^{\prime}} \mathcal{N}_{\Lambda_{\ell_{1}}(r)}^{*}\right\} .
\end{gathered}
$$

Using the probability estimates in (3.16) and (4.44), and taking $K_{1}$ sufficiently large (independently of the scale), we get (4.51). This can be seen as follows. First, using (4.44), we have

$$
\mathbb{P}\left\{\mathcal{E}_{\Lambda_{\ell_{1}}(r)} \cup \mathcal{N}_{\Lambda_{\ell_{1}}(r)}^{*}\right\} \geq \mathbb{P}\left\{\mathcal{N}_{\Lambda_{\ell_{1}}(r)}\right\}>1-L^{-5 \rho_{1} d},
$$

and hence

$$
\begin{aligned}
\mathbb{P}\left\{\bigcap_{r \in R}\left\{\mathcal{E}_{\Lambda_{\ell_{1}}(r)} \cup \mathcal{N}_{\Lambda_{\ell_{1}}(r)}^{*}\right\}\right\} & >1-\left(\frac{2 L}{\ell_{1}}\right)^{d} L^{-5 \rho_{1} d} \\
& \geq 1-2^{d} L^{-\left(6 \rho_{1}-1\right) d}>1-L^{-2 d}
\end{aligned}
$$

for large $L$, where we used (3.52) and (4.39). On the other hand, letting $K_{1}=C^{\prime}\left(K^{\prime}-1\right)$, it follows from (3.16) and (4.39) that

$\mathbb{P}\left\{\right.$ there are $K^{\prime}$ disjoint boxes $\Lambda_{\ell_{1}}(r) \in \mathcal{R}$ with $\left.\omega \notin \mathcal{E}_{\Lambda_{\ell_{1}}(r)}\right\}$

$$
\leq\left(2 L / \ell_{1}\right)^{d K^{\prime}} \ell_{1}^{-p d K^{\prime}} \leq 2^{d K^{\prime}} L^{-d K^{\prime}\left(\rho_{1}(p+1)-1\right)} \leq L^{-2 d},
$$

if $K_{1}>2 C^{\prime} /\left(\rho_{1}(p+1)-1\right)$ and $L$ is large enough. We now take $C^{\prime}=3^{d}-1$, ensuring that the complementary event has at most $K_{1}$ (not necessarily disjoint) boxes $\Lambda_{\ell_{1}}(r) \in \mathcal{R}$ with $\omega \notin \mathcal{E}_{\Lambda_{\ell_{1}}(r)}$. The estimate (4.51) follows from (4.55) and (4.56).

Moreover, it follows from (3.15) and (4.53) that each $\mathcal{Q}\left(R^{\prime}\right)$ is a disjoint union of (non-empty) events of the form

$$
\mathcal{P}_{R^{\prime}}=\left\{\bigcap_{r \in R^{\prime}} \mathcal{C}_{\Lambda_{\ell_{1}}(r)}\right\} \cap\left\{\bigcap_{r \in R \backslash R^{\prime}} \mathcal{N}_{\Lambda_{\ell_{1}}(r)}^{*}\right\},
$$

where $\mathcal{C}_{\Lambda_{\ell_{1}}(r)}$ is a $\left(\Lambda_{\ell_{1}}(r), E, m_{\ell_{1}}, \zeta, \varsigma^{\prime}, S_{\mathcal{C}_{\Lambda_{\ell_{1}}(r)}}\right)$-adapted event for each $r \in R^{\prime}$. Thus $\mathcal{Q}$ is a $\left(\Lambda, E, K_{1}, K_{2}\right)$-ready event.

Given a box $\Lambda$ and a number $Y>0$,

$$
\mathcal{W}_{\Lambda, Y}:=\left\{\omega \in \Omega ;\left\|R_{\omega, \Lambda}(E)\right\| \geq Y\right\}
$$

is a measurable subset of $\Omega$, i.e., an event, and moreover $\mathcal{W}_{\Lambda, Y} \in \mathcal{F}_{\Lambda}$. 
Lemma 4.14. Given a box $\Lambda=\Lambda_{L}$, let $\mathcal{P}$ be a $\left(\Lambda, E, K_{1}, K_{2}\right)$-prepared event, and consider a box $\Lambda_{L_{1}} \subset \Lambda$ with $L_{1}=\left(2 k_{1} \alpha+1\right) \ell_{1}$, constructed as in (3.53) from the standard $\ell_{1}$-covering $\mathcal{R}=\left\{\Lambda_{\ell_{1}}(r)\right\}_{r \in R}$ of $\Lambda$, where $k_{1} \in \mathbb{N}, k_{1} \geq 100 K_{1}$. Then there

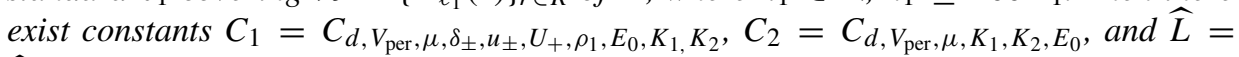
$\widehat{L}_{d, \mu, \delta_{ \pm}, V_{\mathrm{per}}, U_{+}, E_{0}, \rho_{1}, \rho_{2}, \varsigma, \varsigma^{\prime}, K_{1}, K_{2}}$ (the constants are all independent of $k_{1}$ ), such that for all scales $L \geq \widehat{L}$ we have the conditional probability estimate

$$
\mathbb{P}\left\{\left\|R_{\boldsymbol{\omega}, \Lambda_{L_{1}}}(E)\right\| \geq \mathrm{e}^{C_{1} L^{\frac{4}{3} \rho_{1}} \log L} \mid \mathcal{P}\right\} \leq C_{2} L^{-\frac{d}{2}\left(\rho_{1}\left(1-\varsigma^{\prime}\right)-2 \rho_{2}\right)} .
$$

Proof. Let $\mathcal{P}$ be a $\left(\Lambda, E, K_{1}, K_{2}\right)$-prepared event as in (4.47), and let $\left\{\Lambda_{b}\right\}_{b=1, \ldots, B}$ be an enumeration of the notsobad boxes $\left\{\Lambda_{\ell_{1}}(r)\right\}_{r \in R^{\prime \prime} \cap \Lambda_{L_{1}}}$; note $B \leq K_{1}$. For each $b=$ $1, \ldots, B$ we let $\Theta_{b} \subset \Lambda_{b}$ be as in Definition 4.10, so $\left|\Theta_{b}\right| \leq 3^{d} K_{2} \ell_{2}^{d}$. We set $\Theta=$ $\bigcup_{b=1}^{B} \Theta_{b}$, and note $|\Theta| \leq 3^{d} K_{1} K_{2} \ell_{2}^{d}$.

It follows from (3.52) and $k_{1} \geq 100 K_{1}$ that $\#\left(R \cap \Lambda_{L_{1}}\right) \geq\left(200 K_{1}\right)^{d}$, so we can pick distinct $\left\{r_{b}\right\}_{b=1, \ldots, B} \subset R^{\prime} \cap \Lambda_{L_{1}}$ such that for all $b=1, \ldots, B$ we have

$$
4 \ell_{1} \leq \operatorname{dist}\left\{r_{b}, \Lambda_{b}\right\} \leq 12 K_{1} \ell_{1} \quad \text { and } \operatorname{dist}\left\{r_{b}, \bigcup_{b^{\prime}=1}^{B} \Lambda_{b^{\prime}}\right\} \geq 4 \ell_{1} .
$$

Thus, the boxes $\left\{\Lambda_{\ell_{1} / 5}\left(r_{b}\right)\right\}_{b=1, \ldots, B}$ are disjoint, and it follows from (3.14) that for each $b$ we have

$$
\#\left(S_{\mathcal{C}_{\Lambda_{1}\left(r_{b}\right)}} \cap \Lambda_{\ell_{1} / 5}\left(r_{b}\right)\right) \geq N_{1}:=\left[\ell_{1}^{\left(1-\varsigma^{\prime}\right) d}\right] .
$$

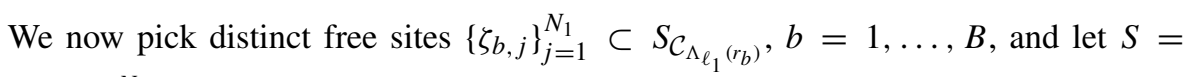
$\bigcup_{b=1}^{B}\left\{\zeta_{b, j}\right\}_{j=1}^{N_{1}}$, so $S \subset S_{\mathcal{P}}$ by (4.50) and we have

$$
\# S=B N_{1} \leq K_{1} \ell_{1}^{\left(1-\varsigma^{\prime}\right) d} .
$$

Given $\boldsymbol{t}_{S}=\left\{t_{\zeta}\right\}_{\zeta \in S} \in[0,1]^{S}$, we consider $H_{\boldsymbol{\omega}, t_{S}, \Lambda_{L_{1}}}$ as in (3.1). We fix $\boldsymbol{\omega} \in \mathcal{P} \in$ $\mathcal{F}_{\Lambda \backslash S_{\mathcal{P}}} \subset \mathcal{F}_{\Lambda \backslash S}$ and set

$$
\widetilde{H}_{t_{S}}=\widetilde{H}_{\omega, t_{S}}=H_{\omega, t_{S}, \Lambda_{L_{1}}} \quad \text { on } \mathrm{L}^{2}\left(\Lambda_{L_{1}}\right) .
$$

Since $\widetilde{H}_{t_{S}} \geq 0$ has compact resolvent, it has non-negative discrete spectrum. Using the min-max principle as in [FK3, Theorem A.1], these eigenvalues (repeated according to the finite multiplicity) are given by

$$
E_{n}\left(\boldsymbol{t}_{S}\right)=\inf _{\mathcal{L} \subset \mathcal{D}\left(\Delta_{\Lambda_{L_{1}}}\right) ; \operatorname{dim} \mathcal{L}=n}\left[\sup _{\psi \in \mathcal{L}:\|\psi\|=1}\left\langle\psi, \widetilde{H}_{t_{S}} \psi\right\rangle\right] \quad \text { for } n \in \mathbb{N} .
$$

Thus, $0 \leq E_{1}\left(t_{S}\right) \leq \cdots \leq E_{n}\left(t_{S}\right) \leq E_{n+1}\left(t_{S}\right) \leq \cdots$, and each $E_{n}\left(\boldsymbol{t}_{S}\right)$ is a continuous function of $\boldsymbol{t}_{S}$, increasing in $t_{\zeta}$ for each $\zeta \in S$. In fact, we have

$$
\left|E_{n}\left(\boldsymbol{t}_{S}\right)-E_{n}\left(\boldsymbol{t}_{S}^{\prime}\right)\right| \leq\left\|V_{t_{S}}-V_{\boldsymbol{t}_{S}^{\prime}}\right\| \leq\left|\boldsymbol{t}_{S}-\boldsymbol{t}_{S}^{\prime}\right|_{1} u_{+}
$$


a general bound that does not take advantage of our construction. To do so, we note that for $\zeta \in S$, each $E_{n}\left(\boldsymbol{t}_{S}\right)$ is piecewise differentiable in $t_{\zeta}$ for fixed $\boldsymbol{t}_{S \backslash\{\zeta\}}$ (cf. [K, Section VII.3.5]), with

$$
\frac{\partial}{\partial t_{\zeta}} E_{n}\left(\boldsymbol{t}_{S}\right)=\left\langle\psi_{n}\left(\boldsymbol{t}_{S}\right), u_{\zeta} \psi_{n}\left(\boldsymbol{t}_{S}\right)\right\rangle
$$

where by $\psi_{n}\left(t_{S}\right)$ we denote a corresponding normalized eigenfunction:

$$
\widetilde{H}_{t_{S}} \psi_{n}\left(\boldsymbol{t}_{S}\right)=E_{n}\left(\boldsymbol{t}_{S}\right) \psi_{n}\left(\boldsymbol{t}_{S}\right), \quad \psi_{n}\left(\boldsymbol{t}_{S}\right) \in \mathcal{D}\left(\Delta_{\Lambda_{L_{1}}}\right) \quad \text { with } \quad\left\|\psi_{n}\left(\boldsymbol{t}_{S}\right)\right\|=1 .
$$

Combining with (2.14), we get

$$
u_{-}\left\|\chi_{\Lambda_{\delta_{-}}(\zeta)} \psi_{n}\left(\boldsymbol{t}_{S}\right)\right\|^{2} \leq \frac{\partial}{\partial t_{\zeta}} E_{n}\left(\boldsymbol{t}_{S}\right) \leq u_{+}\left\|\chi_{\Lambda_{\delta_{+}}(\zeta)} \psi_{n}\left(\boldsymbol{t}_{S}\right)\right\|^{2}
$$

We set $m_{1}=m_{\ell_{1}}$, and consider the intervals

$$
I_{1}=\left[E-\mathrm{e}^{-2 m_{1} \ell_{1}}, E+\mathrm{e}^{-2 m_{1} \ell_{1}}\right] \quad \text { and } \quad I_{2}=\left[E-\mathrm{e}^{-4 m_{1} \ell_{1}}, E+\mathrm{e}^{-4 m_{1} \ell_{1}}\right] .
$$

If $E_{n}\left(t_{S}\right) \in I_{2}$ for some $\boldsymbol{t}_{S} \in[0,1]^{S}$, we can use Lemma 3.9(iii), namely (3.23), to conclude from the upper bound in (4.68), using (4.62), that for all $\boldsymbol{t}_{S}^{\prime} \in[0,1]^{S}$ we have

$$
\left|E_{n}\left(\boldsymbol{t}_{S}^{\prime}\right)-E\right| \leq \mathrm{e}^{-4 m_{1} \ell_{1}}+u_{+} \delta_{+}^{d} K_{1} \ell_{1}^{\left(1-\varsigma^{\prime}\right) d} \mathrm{e}^{-3 m_{1}^{\prime} \ell_{1}} \leq \mathrm{e}^{-2 m_{1} \ell_{1}},
$$

and hence $E_{n}\left(\boldsymbol{t}_{S}^{\prime}\right) \in I_{1}$. In particular, if $\boldsymbol{t}_{S}=0_{S}$ means $t_{\zeta}=0$ for all $\zeta \in S$, we have

$\#\left\{n \in \mathbb{N} ; E_{n}\left(\boldsymbol{t}_{S}\right) \in I_{2}\right.$ for some $\left.\boldsymbol{t}_{S} \in[0,1]^{S}\right\}$

$$
\leq N_{2}:=\#\left\{n \in \mathbb{N} ; E_{n}\left(0_{S}\right) \in I_{1}\right\}=\operatorname{tr}\left\{\chi_{I_{1}}\left(\tilde{H}_{0_{S}}\right)\right\} .
$$

General estimates yield (cf. [GK5, Eq. (A.7)])

$$
N_{2} \leq C_{d, V_{\mathrm{per}}}\left(E+\mathrm{e}^{-2 m_{1} \ell_{1}}\right)^{d / 2} L_{1}^{d} \leq C_{d, V_{\mathrm{per}}, E_{0}} L_{1}^{d},
$$

which is not good enough for our purposes. To improve the estimate, we apply Lemma 3.9(iii). If $E_{n}\left(t_{S}\right) \in I_{1}$, it follows from (3.22) and our construction that

$$
x \notin \Theta \Rightarrow\left\|\chi_{x} \psi_{n}\left(\boldsymbol{t}_{S}\right)\right\| \leq \mathrm{e}^{-\frac{m}{11} \ell_{2}} \leq \mathrm{e}^{-\frac{1}{11} \ell_{2}^{1-\tau}},
$$

and hence, for large $L$,

$$
\left\|\chi_{\Lambda_{L_{1}} \backslash \Theta} \psi_{n}\left(\boldsymbol{t}_{S}\right)\right\| \leq L_{1}^{d} \mathrm{e}^{-\frac{1}{11} \ell_{2}^{1-\tau}} \leq \mathrm{e}^{-\frac{1}{14} \ell_{2}^{1-\tau}} .
$$

It follows that

$$
\operatorname{tr}\left\{\chi_{\Lambda_{L_{1}} \backslash \Theta} \chi_{I_{1}}\left(\tilde{H}_{0_{S}}\right)\right\} \leq \mathrm{e}^{-\frac{1}{7} \ell_{2}^{1-\tau}} N_{2}
$$


Recalling that $\Theta$ is a union of at most $K_{1} K_{2}$ boxes of side $3 \ell_{2}$, and using the trace estimate given in [GK5, Lemma A.4], we obtain

$$
\begin{aligned}
N_{2} & \leq\left(1-\mathrm{e}^{-\frac{1}{7} \ell_{2}^{1-\tau}}\right)^{-1} \operatorname{tr}\left\{\chi_{\Theta} \chi_{I_{1}}\left(\widetilde{H}_{0_{S}}\right)\right\} \\
& \leq 2 \sum_{x \in \mathbb{Z}^{d}} \operatorname{tr}\left\{\chi_{x} \chi_{\Theta} \chi_{I_{1}}\left(\widetilde{H}_{0_{S}}\right)\right\} \leq C_{d, V_{\mathrm{per}}, E_{0}}\left(K_{1} K_{2}\right)^{d} \ell_{2}^{d},
\end{aligned}
$$

a huge improvement over (4.72).

In addition, if $E_{n}\left(t_{S}\right) \in I_{1}$ we conclude from (4.74) that there exists $b^{\prime} \in\{1, \ldots, B\}$ such that

$$
\left\|\chi_{\Theta_{b^{\prime}}} \psi_{n}\left(\boldsymbol{t}_{S}\right)\right\| \geq B^{-1 / 2}\left(1-\mathrm{e}^{-\frac{1}{7} \ell_{2}^{1-\tau}}\right)^{1 / 2} \geq\left(2 K_{1}\right)^{-1 / 2} .
$$

In view of (4.60), it now follows from the quantitative unique continuation principle ([BoK, Lemma 3.10], see Theorem A.1), which we use in the form given in Corollary A.2(i), that

$$
\left\|\chi_{\Lambda_{\delta_{-}}\left(\zeta_{b^{\prime}, j}\right)} \psi_{n}\left(\boldsymbol{t}_{S}\right)\right\| \geq \mathrm{e}^{-C_{3} \ell_{1}^{4 / 3}\left(\log \ell_{1}\right)} \quad \text { for all } j=1, \ldots, N_{1},
$$

with a constant

$$
C_{3}=C_{d, K_{1}, \delta_{-}}\left(1+\left\|V_{\text {per }}\right\|+\delta_{+}^{d} u_{+}+U_{+}+E_{0}\right)^{2 / 3}, \quad \text { where } \quad C_{d, K_{1}, \delta_{-}}>0 .
$$

To exploit (4.78), we set $\zeta_{j}=\left\{\zeta_{b, j}\right\}_{b=1, \ldots, B}$ for $j=1, \ldots, N_{1}$, and let $u_{\zeta_{j}}:=$ $\sum_{b=1}^{B} u_{\zeta b, j}, \chi_{\Lambda_{\delta_{ \pm}}\left(\zeta_{j}\right)}:=\sum_{b=1}^{B} \chi_{\Lambda_{\delta_{ \pm}}\left(\zeta_{b^{\prime}, j}\right)}$. It follows from (4.78) that

$$
\left\|\chi_{\Lambda_{\delta_{-}}\left(\zeta_{j}\right)} \psi_{n}\left(\boldsymbol{t}_{S}\right)\right\| \geq \mathrm{e}^{-C_{3} \ell_{1}^{4 / 3}\left(\log \ell_{1}\right)} \quad \text { for all } j=1, \ldots, N_{1} .
$$

Given $J \subset\left\{1, \ldots, N_{1}\right\}$ we let $S_{J}=\bigcup_{j \in J} \zeta_{j}$.

We now set $\boldsymbol{t}_{j}=\left\{t_{\zeta b, j}\right\}_{b=1, \ldots, B}$ for $j=1, \ldots, N_{1}$, and write $\boldsymbol{t}_{S}=\left\{\boldsymbol{t}_{j}\right\}_{j=1}^{N_{1}}$. Given $j^{\prime}=1, \ldots, N_{1}$, we also define $e_{j}^{\left(j^{\prime}\right)}=\left\{e_{\zeta b, j}^{\left(j^{\prime}\right)}\right\}_{b=1, \ldots, B}$ by $e_{\zeta b, j}^{\left(j^{\prime}\right)}=\delta_{j^{\prime}, j}$ for $b=1, \ldots, B$, $j=1, \ldots, N_{1}$, and let $\boldsymbol{e}_{S}^{\left(j^{\prime}\right)}=\left\{\boldsymbol{e}_{j}^{\left(j^{\prime}\right)}\right\}_{j=1}^{N_{1}}$. It follows, as in (4.66) and (4.68), that for $E_{n}\left(t_{S}\right) \in I_{1}$ we have

$$
u_{-}\left\|\chi_{\Lambda_{\delta_{-}}\left(\zeta_{j}\right)} \psi_{n}\left(\boldsymbol{t}_{S}\right)\right\|^{2} \leq \partial_{j} E_{n}\left(\boldsymbol{t}_{S}\right) \leq u_{+}\left\|\chi_{\Lambda_{\delta_{+}}\left(\zeta_{j}\right)} \psi_{n}\left(\boldsymbol{t}_{S}\right)\right\|^{2},
$$

where

$$
\partial_{j} E_{n}\left(\boldsymbol{t}_{S}\right)=\lim _{s \rightarrow 0} \frac{1}{S}\left(E_{n}\left(\boldsymbol{t}_{S}+s \boldsymbol{e}_{S}^{(j)}\right)-E_{n}\left(\boldsymbol{t}_{S}\right)\right)
$$

so (4.80) yields

$$
\partial_{j} E_{n}\left(\boldsymbol{t}_{S}\right) \geq u_{-} \mathrm{e}^{-2 C_{3} \ell_{1}^{4 / 3}\left(\log \ell_{1}\right)} .
$$

We pick $0 \leq \theta_{-}<\theta_{+} \leq 1$ such that, letting

$$
p_{-}=\mu\left(\left\{\omega \leq \theta_{-}\right\}\right) \quad \text { and } \quad p_{+}=\mu\left(\left\{\omega \geq \theta_{+}\right\}\right),
$$


we have $\left.p_{ \pm} \in\right] 0,1\left[\right.$. ( $\mu$ is the probability distribution in (2.6).) Such $\theta_{ \pm}$always exist since $\mu$ is non-degenerate, and we have $p_{-}+p_{+} \leq 1$. We set $\left.\left.\theta_{\mu}=\theta_{+}-\theta_{-} \in\right] 0,1\right]$.

We now define random variables

$$
\omega_{j}^{+}:=\max _{b=1, \ldots, B} \omega_{\zeta b, j} \quad \text { and } \quad \omega_{j}^{-}:=\min _{b=1, \ldots, B} \omega_{\zeta b, j}, \quad j=1, \ldots, N_{1},
$$

and consider the events

$$
\mathcal{Y}_{j}^{(1)}=\left\{\omega_{j}^{+} \leq \theta_{-}\right\}, \quad \mathcal{Y}_{j}^{(2)}=\left\{\omega_{j}^{-} \geq \theta_{+}\right\}, \quad \mathcal{Y}_{j}^{(0)}=\mathcal{Y}_{j}^{(1)} \sqcup \mathcal{Y}_{j}^{(1)} .
$$

It follows from (4.84) that

$$
p^{(1)}:=\mathbb{P}\left(\mathcal{Y}_{j}^{(1)}\right)=p_{-}^{B}, \quad p^{(2)}:=\mathbb{P}\left(\mathcal{Y}_{j}^{(2)}\right)=p_{+}^{B}, \quad p^{(0)}:=\mathbb{P}\left(\mathcal{Y}_{j}^{(0)}\right)=p^{(1)}+p^{(2)} .
$$

We now introduce Bernoulli random variables $\eta_{j}^{(a)}=\chi_{\mathcal{Y}_{j}^{(a)}}, a=0,1,2$. Then $\eta^{(a)}=\left\{\eta_{j}^{(a)}\right\}_{j=1}^{N_{1}}$ are independent, identically distributed Bernoulli random variables with $\mathbb{P}\left\{\eta_{j}^{(a)}=1\right\}=p^{(a)}$. Note that $\eta_{j}^{(0)}=\eta_{j}^{(1)}+\eta_{j}^{(2)}$, and

$$
\eta_{j}^{(0)}=\eta_{j}^{(1)}+\eta_{j}^{(2)} \quad \text { and } \quad \mathbb{P}\left\{\eta_{j}^{(a)}=1 \mid \eta_{j}^{(0)}=1\right\}=p^{(a)} / p^{(0)}, \quad a=1,2 .
$$

We consider the random index set given by $J_{\eta^{(0)}}=\left\{j \in\left\{1, \ldots, N_{1}\right\} ; \eta_{j}^{(0)}=1\right\}$. Then $\# J_{\boldsymbol{\eta}^{(0)}}=\sum_{j=1}^{N_{1}} \eta_{j}^{(0)}$, and standard large deviation estimates [Ho, Theorem 1] give

$$
\mathbb{P}\left\{\# J_{\boldsymbol{\eta}^{(0)}} \leq \frac{1}{2} N_{1} p^{(0)}\right\} \leq \mathrm{e}^{-\frac{1}{2} N_{1}\left(p^{(0)}\right)^{2}}=\mathrm{e}^{-\frac{1}{2}\left(p_{-}^{B}+p_{+}^{B}\right)^{2} N_{1}} .
$$

Suppose $E_{n}\left(\omega_{S}\right), E_{n}\left(\omega_{S}^{\prime}\right) \in I_{1}$ are such that for some $j$ we have $\omega_{\zeta}=\omega_{\zeta}^{\prime}$ for $\zeta \in$ $S \backslash \zeta_{j}$, and $\eta_{j}^{(1)}\left(\omega_{S}\right)=\eta_{j}^{(2)}\left(\omega_{S}^{\prime}\right)=1$. It then follows from (4.83) that

$$
E_{n}\left(\omega_{S}^{\prime}\right)-E_{n}\left(\omega_{S}\right) \geq u_{-} \theta_{\mu} \mathrm{e}^{-2 C_{3} \ell_{1}^{4 / 3}\left(\log \ell_{1}\right)} .
$$

We set

$$
I=\left[E-\frac{1}{2} u_{-} \theta_{\mu} \mathrm{e}^{-2 C_{3} \ell_{1}^{4 / 3}\left(\log \ell_{1}\right)}, E+\frac{1}{2} u_{-} \theta_{\mu} \mathrm{e}^{-2 C_{3} \ell_{1}^{4 / 3}\left(\log \ell_{1}\right)}\right],
$$

and we will estimate (we write $\boldsymbol{\eta}=\boldsymbol{\eta}^{(0)}$ )

$$
\mathbb{P}_{S}\left\{E_{n}\left(\omega_{S}\right) \in I \mid \eta\right\}=\widehat{\mathbb{E}}_{J_{\eta}}\left\{\widehat{\mathbb{P}}_{J_{\eta}}\left\{E_{n}\left(\omega_{S}\right) \in I\right\}\right\},
$$

where, given $J \subset\left\{1, \ldots, N_{1}\right\}$, we write

$$
\widehat{\mathbb{P}}_{J}\{\cdot\}:=\mathbb{P}_{S_{J}}\left\{\cdot \mid \eta_{j}=1, j \in J\right\}, \quad \widehat{\mathbb{E}}_{J}\{\cdot\}:=\mathbb{E}_{S \backslash S_{J}}\left\{\cdot \mid \eta_{j}=0, j \notin J\right\} .
$$

It follows from (4.88) that, with respect to $\widehat{\mathbb{P}}_{J}, \boldsymbol{\eta}_{J}^{(2)}=\left\{\eta_{j}^{(2)}\right\}_{j \in J}$ is a family of independent identically distributed Bernoulli random variables with

$$
\widehat{\mathbb{P}}_{J}\left\{\eta_{j}^{(2)}=1\right\}=\frac{p^{(2)}}{p^{(0)}}=\frac{p_{+}^{B}}{p_{-}^{B}+p_{+}^{B}}, \quad j \in J .
$$


The configuration space of $\eta_{J}^{(2)},\{0,1\}^{J}$, is partially ordered by the relation defined by $\boldsymbol{\varepsilon} \prec \boldsymbol{\varepsilon}^{\prime} \Leftrightarrow \varepsilon_{j} \leq \varepsilon_{j}^{\prime}$ for all $\in J$. Let us write $\omega_{S}=\left(\boldsymbol{\omega}_{S \backslash S_{J}}, \boldsymbol{\omega}_{S_{J}}\right)$. For a fixed $\omega_{S \backslash S_{J}}$ we set

$$
\mathcal{A}_{\omega_{S \backslash S_{J}}}=\left\{\eta_{J}^{(2)}\left(\omega_{S \backslash S_{J}}, \omega_{S_{J}}\right) ; E_{n}\left(\omega_{S \backslash S_{J}}, \omega_{S_{J}}\right) \in I\right\} \subset\{0,1\}^{J} .
$$

It follows from (4.90) and (4.91) that $\mathcal{A}_{\omega_{S \backslash S_{J}}}$ is an anti-chain in $\{0,1\}^{J}$, i.e., if $\boldsymbol{\varepsilon}, \boldsymbol{\varepsilon}^{\prime} \in$ $\mathcal{A}_{\omega_{S \backslash S_{J}}}$ and $\boldsymbol{\varepsilon} \prec \boldsymbol{\varepsilon}^{\prime}$, then $\boldsymbol{\varepsilon}=\boldsymbol{\varepsilon}^{\prime}$. Using the probabilistic Sperner Lemma given in [AGKW, Lemma 3.1] with (4.94), we get

$$
\widehat{\mathbb{P}}_{J}\left\{E_{n}\left(\omega_{S \backslash S_{J}}, \omega_{S_{J}}\right) \in I\right\}=\widehat{\mathbb{P}}_{J}\left\{\eta_{J}^{(2)} \in \mathcal{A}_{\omega_{S \backslash S_{J}}}\right\} \leq \frac{2 \sqrt{2}\left(p_{-}^{B}+p_{+}^{B}\right)}{\left(p_{-} p_{+}\right)^{B / 2} \sqrt{\# J}} .
$$

It follows from (4.92) and (4.96) that

$$
\mathbb{P}_{S}\left\{E_{n}\left(\omega_{S}\right) \in I \mid \eta\right\} \leq \frac{2 \sqrt{2}}{\left(p_{-} p_{+}\right)^{K_{1} / 2} \sqrt{\# J_{\eta}}} .
$$

Combining (4.89) and (4.97) we obtain

$$
\begin{aligned}
\mathbb{P}_{S}\left\{E_{n}\left(\omega_{S}\right) \in I\right\} & \leq \mathrm{e}^{-\frac{1}{2}\left(p_{-}^{B}+p_{+}^{B}\right)^{2} N_{1}}+4\left(p_{-} p_{+}\right)^{-B / 2}\left(p_{-}^{B}+p_{+}^{B}\right)^{1 / 2} N_{1}^{-1 / 2} \\
& \leq \mathrm{e}^{-\frac{1}{2}\left(p_{-}^{K_{1}}+p_{+}^{K_{1}}\right)^{2} N_{1}}+4\left(p_{-} p_{+}\right)^{-K_{1} / 2} N_{1}^{-1 / 2} \\
& \leq C_{\mu, K_{1}} \ell_{1}^{-\frac{1}{2}\left(1-\varsigma^{\prime}\right) d} .
\end{aligned}
$$

We now conclude from (4.98), (4.71), and (4.76) that

$$
\begin{aligned}
\mathbb{P}_{S}\left\{\left\|\left(\widetilde{H}_{\omega_{S}}-E\right)^{-1}\right\| \geq 2\left(u_{-} \theta_{\mu}\right)^{-1} \mathrm{e}^{2 C_{3} \ell_{1}^{4 / 3}\left(\log \ell_{1}\right)}\right\} & =\mathbb{P}_{S}\left\{\sigma\left(\widetilde{H}_{\omega_{S}}\right) \cap I \neq \emptyset\right\} \\
& \leq C_{4} \ell_{1}^{-\frac{1}{2}\left(1-\varsigma^{\prime}\right) d} \ell_{2}^{d},
\end{aligned}
$$

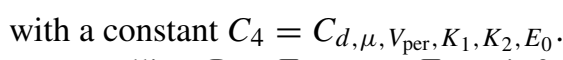

Recalling $\mathcal{P} \in \mathcal{F}_{\Lambda \backslash S_{\mathcal{P}}} \subset \mathcal{F}_{\Lambda \backslash S}$, it follows from (4.99) that

$$
\begin{aligned}
\mathbb{P}\left\{\left\{\left\|R_{\boldsymbol{\omega}, \Lambda_{L_{1}}}(E)\right\|\right.\right. & \left.\left.\geq 2\left(u_{-} \theta_{\mu}\right)^{-1} \mathrm{e}^{2 C_{3} \ell_{1}^{4 / 3}\left(\log \ell_{1}\right)}\right\} \cap \mathcal{P}\right\} \\
& =\mathbb{P}\left\{\chi_{\mathcal{P}}(\omega) \mathbb{P}_{S}\left\{\left\|R_{\boldsymbol{\omega}, \Lambda_{L_{1}}}(E)\right\| \leq 2\left(u_{-} \theta_{\mu}\right)^{-1} \mathrm{e}^{2 C_{3} \ell_{1}^{4 / 3}\left(\log \ell_{1}\right)}\right\}\right\} \\
& \leq C_{4} \ell_{1}^{-\frac{1}{2}\left(1-\varsigma^{\prime}\right) d} \ell_{2}^{d} \mathbb{P}\{\mathcal{P}\},
\end{aligned}
$$

which yields (4.59).

Lemma 4.15. Given a box $\Lambda=\Lambda_{L}$, let $\mathcal{P}$ be a $\left(\Lambda, E, K_{1}, K_{2}\right)$-prepared event. Then, if $L$ is large enough, depending only on $d, \mu, \delta_{ \pm}, V_{\mathrm{per}}, U_{+}, p, E_{0}, \rho_{1}, \rho_{2}, \varsigma, \varsigma^{\prime}, \tau, K_{1}, K_{2}$, there exists an event $\mathcal{W}_{\mathcal{P}} \subset \mathcal{P}$, with

$$
\mathbb{P}\left\{\mathcal{W}_{\mathcal{P}}\right\} \leq C_{2} K_{1} L^{-\frac{d}{2}\left(\rho_{1}\left(1-\varsigma^{\prime}\right)-2 \rho_{2}\right)} \mathbb{P}\{\mathcal{P}\},
$$


where the constant $C_{2}$ is as in (4.59), such that the event $\mathcal{P} \backslash \mathcal{W}_{\mathcal{P}}$ is $\left(\Lambda, E, m_{L}, \varsigma, \varsigma^{\prime}\right)$ adapted with

$$
\begin{aligned}
m_{L} & =m_{\ell_{1}}\left(1-C_{d, V_{\mathrm{per}}, E_{0}, K_{1}} L^{-\beta}\right) \geq L^{-\tau}, \quad \text { where } \\
\beta & =\min \left\{\left(\frac{4}{3} \varsigma(1-\varsigma)^{-1}-\tau\right) \rho_{1}, \frac{1}{2}\left(1-\frac{4}{3}(1-\varsigma)^{-1} \rho_{1}\right)\right\}>0 .
\end{aligned}
$$

Proof. Let $\mathcal{P}$ be a $\left(\Lambda, E, K_{1}, K_{2}\right)$-prepared event as in (4.47). We take

$$
\kappa=\frac{1}{2}\left(1+\frac{4}{3} \rho_{1}(1-\varsigma)^{-1}\right), \quad \text { so } \quad \frac{4}{3} \rho_{1}(1-\varsigma)^{-1}<\kappa<1,
$$

where we used (4.39), and we have

$$
\kappa \varsigma-\tau \rho_{1}>\left(\frac{4}{3} \varsigma(1-\varsigma)^{-1}-\tau\right) \rho_{1} .
$$

By geometrical considerations, we can find disjoint boxes $\left\{\Lambda_{j}\right\}_{j=1}^{J}, J \leq \# R^{\prime \prime} \leq K_{1}$, where each $\Lambda_{j}=\Lambda_{L_{j}} \subset \Lambda$ is constructed as in (3.53) from the standard $\ell_{1}$-covering $\mathcal{R}=\left\{\Lambda_{\ell_{1}}(r)\right\}_{r \in R}$ of $\Lambda$ with $L^{\kappa} \leq L_{j} \leq K_{1} L^{\kappa}$, and for every $r \in R^{\prime \prime}$ there exists a (unique) $j_{r} \in\{1, \ldots, J\}$ with $\Lambda_{\ell_{1}(r)} \subset \Lambda_{j_{r}}^{\left(\Lambda, L^{\kappa} / 10\right)}$. Since it follows from (4.103) that (for $L$ large enough)

$$
\mathrm{e}^{C_{1} L^{\frac{4}{3} \rho_{1}} \log L} \leq \mathrm{e}^{L^{\kappa(1-\varsigma)}}
$$

we conclude from Lemma 4.14 that for all $j=1, \ldots, J$, letting

$$
\mathcal{W}_{j}=\left\{\left\|R_{\omega, \Lambda_{j}}(E)\right\| \geq \mathrm{e}^{L^{\kappa(1-\varsigma)}}\right\} \cap \mathcal{P},
$$

we have

$$
\mathbb{P}\left\{\mathcal{W}_{j}\right\} \leq C_{2} L^{-\frac{d}{2}\left(\rho_{1}\left(1-\varsigma^{\prime}\right)-2 \rho_{2}\right)} \mathbb{P}\{\mathcal{P}\}
$$

We set $\mathcal{W}_{\mathcal{P}}=\bigcup_{j=1}^{J} \mathcal{W}_{j} \subset \mathcal{P}$, so (4.101) holds.

Since $\mathcal{P}$ is a $\left(\Lambda, E, K_{1}, K_{2}\right)$-prepared event, the hypotheses of Lemma 3.10 are satisfied for $\omega \in \mathcal{P} \backslash \mathcal{W}_{\mathcal{P}}$, so we conclude that the box $\Lambda$ is $\left(\boldsymbol{\omega}, E, m_{L}, \varsigma\right)$-good for all $\omega \in \mathcal{P} \backslash \mathcal{W}_{\mathcal{P}}$ with $m_{L}$ as in (4.102).

Moreover, for all $j$ we have $\left\{\left\|R_{\omega, \Lambda_{j}}(E)\right\| \geq \mathrm{e}^{L^{\kappa(1-s)}}\right\} \in \mathcal{F}_{\Lambda_{j}}$, so it follows from (4.47) that $\mathcal{W}_{j} \in \mathcal{F}_{\Lambda_{j}^{\prime}}$, where $\Lambda_{j}^{\prime}=\left\{x \in \Lambda\right.$; $\left.\operatorname{dist}\left(x, \Lambda_{j}\right)<\ell_{1}\right\}$. Let $\Lambda^{\prime}=\bigcup_{j=1}^{J} \Lambda_{j}^{\prime}$. It follows that $S_{\mathcal{P} \backslash \mathcal{W}_{\mathcal{P}}}:=S_{\mathcal{P}} \backslash \Lambda^{\prime}$ consists of free sites for $\mathcal{P} \backslash \mathcal{W}_{\mathcal{P}}$, i.e., the box $\Lambda$ is

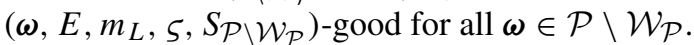

To conclude that $\mathcal{P} \backslash \mathcal{W}_{\mathcal{P}}$ is $\left(\Lambda, E, m_{L}, \varsigma, \varsigma^{\prime}\right)$-adapted we only need to show that $S_{\mathcal{P} \backslash \mathcal{W}_{\mathcal{P}}}$ is $\varsigma^{\prime}$-abundant. This can be done as in Lemma 3.9(i). Since

$$
\bigsqcup_{r \in R^{\prime} \backslash \Lambda^{\prime}} S_{\mathcal{C}_{\Lambda_{\ell_{1}}(r)}} \cap \Lambda_{\ell_{1} / 5}(r) \subset S_{\mathcal{P} \backslash \mathcal{W}_{\mathcal{P}}},
$$

it follows, using (3.14), that for all boxes $\Lambda_{L / 5} \subset \Lambda$ we have (for $L$ sufficiently large)

$$
\begin{aligned}
\#\left(S_{\left.\mathcal{P} \backslash \mathcal{W}_{\mathcal{P}} \cap \Lambda_{L / 5}\right)}\right. & \geq \ell_{1}^{\left(1-\varsigma^{\prime}\right) d}\left(\left(\frac{5}{4} \frac{L}{5 \ell_{1}}-2\right)^{d}-K_{1}\left(\frac{5}{3} \frac{K_{1} L^{\kappa}}{\ell_{1}}\right)^{d}\right) \\
& \geq L^{\left(1-\varsigma^{\prime}\right) d},
\end{aligned}
$$

and hence $S_{\mathcal{P} \backslash \mathcal{W}_{\mathcal{P}}}$ is a $\varsigma^{\prime}$-abundant subset of $\Lambda$. 
Lemma 4.16. Suppose $\left\{L_{n} ; n=0,1, \ldots, n_{1}\right\}$ is $\left(E, \varsigma, \varsigma^{\prime}, p, \tau\right)$-extra good. Then, if $L$ is sufficiently large, depending only on $d, \mu, \delta_{ \pm}, V_{\mathrm{per}}, U_{+}, p, E_{0}, \rho_{1}, \rho_{2}, \varsigma, \varsigma^{\prime}, \tau$, the scale $L$ is $\left(E, m_{L}, \varsigma, \varsigma^{\prime}, p\right)$-extra good, and

$$
m_{L}=m_{\ell_{1}}\left(1-C_{d, V_{\mathrm{per}}, E_{0}, \rho_{1}, \rho_{2}} L^{-\beta}\right) \geq L^{-\tau},
$$

where $\beta$ is given in (4.102).

Proof. Since by hypothesis $\left\{L_{n} ; n=0,1, \ldots, n_{1}\right\}$ is $\left(E, \varsigma, \varsigma^{\prime}, p, \tau\right)$-extra good, it follows from Lemma 4.13 that there exist $K_{1}, K_{2} \in \mathbb{N}$ such that, given a box $\Lambda=\Lambda_{L}$, if $L$ is sufficiently large there exists a $\left(\Lambda, E, K_{1}, K_{2}\right)$-ready event $\mathcal{Q}$ satisfying (4.51). We write $\mathcal{Q}$ as in (4.49), and apply Lemma 4.15 to any $\left(\Lambda, E, K_{1}, K_{2}\right)$-prepared events $\mathcal{P}_{j}$, letting $\mathcal{W}_{\mathcal{P}_{j}}$ denote the corresponding event. In particular, $\mathcal{W}_{\mathcal{P}_{j}}$ satisfies (4.101) and $\mathcal{P}_{j} \backslash \mathcal{W}_{\mathcal{P}_{j}}$ is a $\left(\Lambda, E, m_{L}, \zeta, \varsigma^{\prime}\right)$-adapted event with $m_{L}$ is as in (4.102), which yields (4.110) since $K_{1}, K_{2}$ depend only on $d, p, \rho_{1}, \rho_{2}$. It then follows that

$$
\mathcal{E}=\bigsqcup_{j=1}^{J}\left(\mathcal{P}_{j} \backslash \mathcal{W}_{\mathcal{P}_{j}}\right)=\mathcal{Q} \backslash\left(\bigcup_{j=1}^{J} \mathcal{W}_{\mathcal{P}_{j}}\right)
$$

is a $\left(\Lambda_{L}, E, m_{L}, \varsigma, \varsigma^{\prime}\right)$-extra good event. Since it follows from (4.49) and (4.101) that

$$
\mathbb{P}\left\{\bigcup_{j=1}^{J} \mathcal{W}_{\mathcal{P}_{j}}\right\} \leq C_{2} K_{1} L^{-\frac{d}{2}\left(\rho_{1}\left(1-\varsigma^{\prime}\right)-2 \rho_{2}\right)} \mathbb{P}\{\mathcal{Q}\},
$$

we get, using (4.51) and (4.39),

$$
\mathbb{P}\{\mathcal{E}\} \geq\left(1-2 L^{-2 d}\right)\left(1-C_{2} K_{1} L^{-\frac{d}{2}\left(\rho_{1}\left(1-\varsigma^{\prime}\right)-2 \rho_{2}\right)}\right) \geq 1-L^{-p d} .
$$

We can now finish the proof of Proposition 4.6.

Proof of Proposition 4.6. Let $E \in\left[0, E_{0}\right]$ and suppose that for some scale $L_{0}$ we know that $L$ is $\left(E, m_{0}, \varsigma, \varsigma^{\prime}, p\right)$-extra good for all $L \in\left[L_{0}, L_{0}^{\rho_{1}^{-1} \rho_{2}^{-1}}\right]$, with $m_{0}$ satisfying (4.40). In other words, the interval $\left[L_{0}, L_{0}^{\rho_{1}^{-1} \rho_{2}^{-1}}\right]$ is $\left(E, \varsigma, \varsigma^{\prime}, p, \tau\right)$-extra good with $m_{L}=m_{0}$ for $L \in\left[L_{0}, L_{0}^{\rho_{1}^{-1} \rho_{2}^{-1}}\right]$. We also assume that $L_{0}$ is large enough so we can use Lemma 4.16 for all $L \geq L_{0}$.

$$
\begin{gathered}
\text { Let } \mathcal{L}_{0}=\left[L_{0}, L_{0}^{\rho_{1}^{-1} \rho_{2}^{-1}}\right] \text { and } \mathcal{L}_{k}=\left[L_{0}^{\rho_{1}^{-k} \rho_{2}^{-1}, L_{0}^{\rho_{1}^{-(k+1)}} \rho_{2}^{-1}}\right] \text { for } k=1,2, \ldots \text { We set } \\
\qquad m_{k}=m_{0} \prod_{k^{\prime}=1}^{k}\left(1-C_{E_{0}} L_{0}^{-\beta \rho_{1}^{-k^{\prime}} \rho_{2}^{-1}}\right) \geq L_{0}^{-\tau \beta \rho_{1}^{-k} \rho_{2}^{-1}}
\end{gathered}
$$

where $C_{E_{0}}=C_{d, V_{\mathrm{per}}, \rho_{1}, \rho_{2}, E_{0}}$ and $\beta$ are as in (4.110), the inequality holding for all $k$ by taking $L_{0}$ sufficiently large. We consider statements $\left(\mathcal{S}_{k}\right)$, given for $k=0,1, \ldots$ by:

$\left(\mathcal{S}_{k}\right)$ The scale interval $\mathcal{L}_{k}$ is $\left(E, \varsigma, \varsigma^{\prime}, p, \tau\right)$-extra good with $m_{L} \geq m_{k}$ for all $L \in \mathcal{L}_{k}$. 
We will prove that $\left(\mathcal{S}_{k}\right)$ is valid for all $k=0,1, \ldots$ by induction. Note that the validity of $\left(\mathcal{S}_{0}\right)$ is our hypothesis, and $\left(\mathcal{S}_{1}\right)$ follows immediately from $\left(\mathcal{S}_{0}\right)$ by Lemma 4.16. If $k=1,2, \ldots$, and $\left(\mathcal{S}_{k-1}\right)$ and $\left(\mathcal{S}_{k}\right)$ are valid, we can apply Lemma 4.16 for all $L \in \mathcal{L}_{k+1}$, and conclude that $\left(\mathcal{S}_{k+1}\right)$ holds with

$$
m_{L} \geq m_{k}\left(1-C_{E_{0}} L^{-\beta}\right) \geq m_{k+1} \geq L_{0}^{-\tau \beta \rho_{1}^{-(k+1)} \rho_{2}^{-1}} \geq L^{-\tau}
$$

Since we have $\left(\mathcal{S}_{k}\right)$ for all $k=0,1, \ldots$, we conclude that the scale interval $\left[L_{0}, \infty\right.$ [ $=\bigcup_{k=0}^{\infty} \mathcal{L}_{k}$ is $\left(E, \varsigma, \varsigma^{\prime}, p, \tau\right)$-extra good, and for all $L \in\left[L_{0}, \infty[\right.$ we have

$$
m_{L} \geq m_{0} \prod_{k=1}^{\infty}\left(1-C_{E_{0}} L_{0}^{-\beta \rho_{1}^{-k} \rho_{2}^{-1}}\right) \geq m_{0} / 2
$$

for sufficiently large $L_{0}$. In particular, every scale $L \geq L_{0}$ is $\left(E, m_{0} / 2, \varsigma, \varsigma^{\prime}, p\right)$-extra good, so the theorem is proved.

\section{Preamble to localization}

In this section we introduce tools for extracting localization from the multiscale analysis.

Let $v>d / 2$. (We will work with a fixed $v$ that will be generally omitted from the notation.) Given $y \in \mathbb{R}^{d}$, we recall that $T_{y}=T_{\nu, y}$ denotes the operator on the Hilbert space $\mathcal{H}=\mathrm{L}^{2}\left(\mathbb{R}^{d}\right)$ given by multiplication by the function $T_{y}(x)=T_{v, y}(x):=\langle x-y\rangle^{v}$ for $x \in \mathbb{R}^{d}$ with $T:=T_{0}$. Since $\left\langle y_{1}+y_{2}\right\rangle \leq \sqrt{2}\left\langle y_{1}\right\rangle\left\langle y_{2}\right\rangle$, we have

$$
\left\|T_{y_{1}} T_{y_{2}}^{-1}\right\| \leq 2^{v / 2}\left\langle y_{1}-y_{2}\right\rangle^{v} .
$$

The domain of $T, \mathcal{D}(T)$, equipped with the norm $\|\phi\|_{+}=\|T \phi\|$, is a Hilbert space, denoted by $\mathcal{H}_{+}=\mathcal{H}_{v,+}$. The Hilbert space $\mathcal{H}_{-}=\mathcal{H}_{v,-}$ is defined as the completion of $\mathcal{H}$ in the norm $\|\psi\|_{-}=\left\|T^{-1} \psi\right\|$. By construction, $\mathcal{H}_{+} \subset \mathcal{H} \subset \mathcal{H}_{-}$, and the natural injections $l_{+}: \mathcal{H}_{+} \rightarrow \mathcal{H}$ and $\iota_{-}: \mathcal{H} \rightarrow \mathcal{H}_{-}$are continuous with dense range. The operators $T_{+}: \mathcal{H}_{+} \rightarrow \mathcal{H}$ and $T_{-}: \mathcal{H} \rightarrow \mathcal{H}_{-}$, defined by $T_{+}=T l_{+}$, and $T_{-}=l_{-} T$ on $\mathcal{D}(T)$, are unitary. Note that it follows from (5.1) that

$$
\left\|T_{y}^{-1} \psi\right\| \leq 2^{v / 2}\langle y\rangle^{v}\left\|T^{-1} \psi\right\| \quad \text { for all } y \in \mathbb{R}^{d} \text { and } \psi \in \mathcal{H}_{-} .
$$

\section{1. v-generalized eigenfunctions}

Let $H_{\omega}$ be a generalized Anderson Hamiltonian. For a fixed $\omega \in \Omega$ we now consider only generalized eigenfunctions $\psi \in \mathcal{H}_{-}=\mathcal{H}_{v,-}$, so we rewrite Definition 2.3 as follows.

Definition 5.1. A v-generalized eigenfunction for $H_{\omega}$ with generalized eigenvalue $E$ is a function $\psi \in \mathcal{H}_{v,-}$ such that $\psi \neq 0$ and

$$
\left\langle H_{\omega} \varphi, \psi\right\rangle=E\langle\varphi, \psi\rangle \quad \text { for all } \varphi \in C_{c}^{\infty}\left(\mathbb{R}^{d}\right) .
$$


Given $E \in \mathbb{R}$ we let $\Theta_{\omega}(E)=\Theta_{\nu, \omega}(E)$ denote the collection of all $\nu$-generalized eigenfunctions for $H_{\omega}$ with generalized eigenvalue $E$, and set $\widetilde{\Theta}_{\omega}(E)=\Theta_{\omega}(E) \cup\{0\}$. We will drop $v$ from the notation: $\psi$ will be called a generalized eigenfunction for $H_{\omega}$ with generalized eigenvalue $E$ if and only if $\psi \in \Theta_{\omega}(E)$. We will also call $E \in \mathbb{R}$ a generalized eigenvalue for $H_{\omega}$ if and only if $\Theta_{\omega}(E) \neq \emptyset$.

The generalized eigenvalues and eigenfunctions of $H_{\omega}$ are the same as the eigenvalues and eigenfunctions of the operator $H_{\omega,-}$ : a function $\psi \in \mathcal{H}_{-}, \psi \neq 0$, is a generalized eigenfunction of $H_{\omega}$ with generalized eigenvalue $E$ if and only if $\psi \in \mathcal{D}\left(H_{\omega,-}\right)$ and $H_{\omega,-} \psi=E \psi$, i.e.,

$$
\left\langle H_{\omega} \phi, \psi\right\rangle=E\langle\phi, \psi\rangle \quad \text { for all } \phi \in \mathcal{D}\left(H_{\omega}\right) \cap \mathcal{H}_{+} .
$$

This follows from the fact that (5.4) is equivalent to (5.3) since $C_{c}^{\infty}\left(\mathbb{R}^{d}\right)$ is a core for the $H_{\omega}$.

Eigenvalues and eigenfunctions of $H_{\omega}$ are always generalized eigenvalues and eigenfunctions. Conversely, if $\psi \in \Theta_{\omega}(E) \cap \mathcal{H}$, i.e., $\psi \in \mathcal{H}$ is a generalized eigenfunction of $H_{\omega}$ with generalized eigenvalue $E$, then $\psi$ is an eigenfunction of $H_{\omega}$ with eigenvalue $E$.

\subsection{Generalized eigenfunctions and good boxes}

Given $\omega \in \Omega, x \in \mathbb{R}^{d}$ and $E \in \mathbb{R}$, we set

$$
W_{\boldsymbol{\omega}, x}(E)=W_{\omega, x}^{(v)}(E):= \begin{cases}\sup _{\psi \in \Theta_{\omega}(E)} \frac{\left\|\chi_{x} \psi\right\|}{\left\|T_{x}^{-1} \psi\right\|} & \text { if } \Theta_{\omega}(E) \neq \emptyset \\ 0 & \text { otherwise. }\end{cases}
$$

Note that

$$
W_{\omega, x}(E) \leq(5 / 4)^{v / 2}<2^{v / 2} .
$$

Remark 5.2. By the unique continuation principle, $\Theta_{\omega}(E) \neq \emptyset$ if and only if $W_{\omega, x}(E)$ $\neq 0$ for all $x \in \mathbb{R}$.

Lemma 5.3. Let $\omega \in \Omega, I \subset \mathbb{R}$ a bounded interval, $E \in I, 0<\varsigma<1$, and $m>0$. Suppose the box $\Lambda_{L}(x)$ is $(\omega, E, m, \varsigma)$-jgood. Then, if $m \geq C_{d, v, V_{\mathrm{per}}, I} \frac{\log L}{L}$, we have

$$
W_{\omega, y}(E) \leq \mathrm{e}^{-\frac{m}{15} L} \quad \text { for all } y \in \Lambda_{L}(x) \text { with } \Lambda_{L / 5}(y) \subset \Lambda_{L}(x) .
$$

Proof. We can assume $\Theta_{\omega}(E) \neq \emptyset$. Given $\psi \in \Theta_{\omega}(E)$, it follows from Lemma 2.5 that for all $y \in \Lambda_{L}(x)$ with $\Lambda_{L / 5}(y) \subset \Lambda_{L}(x)$ we have

$$
\left\|\chi_{y} \psi\right\| \leq 2 \gamma_{E} L^{d-1} \mathrm{e}^{-\frac{m}{11} L} \max _{y^{\prime} \in \Upsilon_{\Lambda_{L}(x)}}\left\|\chi_{y^{\prime}} \psi\right\| \leq 2 \gamma_{E} L^{d-1}\left(1+L^{2}\right)^{v / 2} \mathrm{e}^{-\frac{m}{11} L}\left\|T_{y}^{-1} \psi\right\|,
$$

so

$$
\frac{\left\|\chi_{y} \psi\right\|}{\left\|T_{y}^{-1} \psi\right\|} \leq \mathrm{e}^{-\frac{m}{15} L} \quad \text { for } m \geq C_{d, \nu, V_{\mathrm{per}}, I} \frac{\log L}{L}
$$




\subsection{Generalized eigenfunctions and annuli of good boxes}

Given $\omega \in \Omega, x \in \mathbb{R}^{d}, E \in \mathbb{R}$, and a scale $L$, we set (cf. (1.8))

$$
W_{\boldsymbol{\omega}, x, L}(E)=W_{\omega, x, L}^{(\nu)}(E):= \begin{cases}\sup _{\psi \in \Theta_{\omega}(E)} \frac{\left\|\chi_{x, L} \psi\right\|}{\left\|T_{x}^{-1} \psi\right\|} & \text { if } \Theta_{\omega}(E) \neq \emptyset \\ 0 & \text { otherwise. }\end{cases}
$$

where $\chi_{x, L}:=\chi_{\Lambda_{2 L+1, L-1}(x)}$, and

$$
L_{-}:=L-\frac{1}{5} \frac{L}{100}=\frac{499}{500} L, \quad L_{+}:=2 L+\frac{1}{5} \frac{L}{100}=\frac{1001}{500} L .
$$

In particular, we have $(L \geq 2)$

$$
W_{\omega, x, L}(E) \leq\left(1+(L+1 / 2)^{2}\right)^{v / 2} \leq 2^{v / 2} L^{v} .
$$

Note also that, using (5.1),

$$
\begin{aligned}
W_{\boldsymbol{\omega}, y}(E) & \leq 2^{v / 2}\langle y-x\rangle^{\nu} W_{\boldsymbol{\omega}, x, L}(E) \\
& \leq 2^{v} L^{\nu} W_{\boldsymbol{\omega}, x, L}(E) \quad \text { for } y \in \bar{\Lambda}_{2 L, L}(x) .
\end{aligned}
$$

Lemma 5.4. Let $\omega \in \Omega, I \subset \mathbb{R}$ a bounded interval, $E \in I, 0<\varsigma<1,0<\widetilde{m}<m$. Suppose every box $\Lambda_{L / 100}$ in the standard L/100-covering of the annulus $\Lambda_{L_{+}, L_{-}}(x)$ is $(\omega, E, m, \varsigma)$-jgood. Then, if $m \geq C_{d, v, V_{\mathrm{per}}, I}^{\prime} \frac{\log L}{L}$, we have

$$
W_{\boldsymbol{\omega}, x, L}(E) \leq \mathrm{e}^{-\frac{m}{2000} L} .
$$

Proof. We can assume $\Theta_{\omega}(E) \neq \emptyset$. Given $y \in \bar{\Lambda}_{2 L, L}(x)$ there exists a box $\Lambda_{L / 100}^{(y)}$ in the standard $L / 100$-covering of the annulus $\Lambda_{L_{+}, L_{-}}(x)$ with $\Lambda_{L / 500}(y) \subset \Lambda_{L / 100}^{(y)}$. Since the box $\Lambda_{L / 100}^{(y)}$ is $(\omega, E, m, \varsigma)$-jgood by hypothesis, it follows from Lemma 5.3 that for all $\psi \in \Theta_{\omega}(E)$ we have, with $\ell=L / 100$ and $m \geq C_{d, v, V_{\mathrm{per}}, I} \frac{\log \ell}{\ell}$,

$$
\begin{aligned}
\left\|\chi_{y} \psi\right\| & \leq\left\|T_{y}^{-1} \psi\right\| \mathrm{e}^{-\frac{m}{15} \ell} \leq 2^{v / 2}\langle y-x\rangle^{v}\left\|T_{x}^{-1} \psi\right\| \mathrm{e}^{-\frac{m}{15} \ell} \\
& \leq 2^{v} L^{v}\left\|T_{x}^{-1} \psi\right\| \mathrm{e}^{-\frac{m}{15} \ell} .
\end{aligned}
$$

It follows that

$$
\left\|\chi_{x, L} \psi\right\| \leq C_{d, \nu} L^{\nu+d}\left\|T_{x}^{-1} \psi\right\| \mathrm{e}^{-\frac{m}{15} \ell} \leq\left\|T_{x}^{-1} \psi\right\| \mathrm{e}^{-\frac{m}{20} \ell}
$$

which yields (5.14). 


\subsection{Generalized eigenfunction expansion}

A generalized Anderson Hamiltonian $H_{\omega}$ has a generalized eigenfunction expansion, which we will now review. We follow [KIKS, Section 3], to which we refer for all the details. (Although the results in [KIKS] are stated for classical wave operators, which include $-\Delta$, they clearly hold for $-\Delta+V$ with $V$ a bounded potential; in particular they hold for generalized Anderson Hamiltonians as in Definition 2.2.)

Let $H_{\omega}$ be a generalized Anderson Hamiltonian. For all $\omega \in \Omega$ we have the estimate (e.g., [GK5, Lemma A.4])

$$
\operatorname{tr}\left\{T^{-1}\left(H_{\omega}+1+\left\|V_{\text {per }}^{-}\right\|\right)^{-2[[d / 4]]} T^{-1}\right\} \leq C_{d, v,\left\|V_{\text {per }}^{-}\right\|}<\infty,
$$

where $[[d / 4]]=\min \{n \in \mathbb{N} ; n>d / 4\}$ and $V_{\text {per }}^{-}$is the negative part of $V_{\text {per. }}$. We define the spectral measure

$$
\left.\mu_{\omega}(B):=\operatorname{tr}\left\{T^{-1} P_{\omega}(B) T^{-1}\right\}=\| T^{-1} P_{\omega}(B)\right) \|_{2}^{2}, \quad B \subset \mathbb{R} \text { a Borel set. }
$$

As a consequence of (5.17), for all Borel sets $B$ with $\sup B<\infty$ we have

$$
\mu_{\omega}(B) \leq C_{d, v,\left\|V_{\operatorname{per}}^{-}\right\|, \sup B}<\infty \quad \text { for all } \omega \in \Omega .
$$

Moreover, since the constants in (5.17) and (5.19) depend on the potential only through $\left\|V_{\text {per }}^{-}\right\|$(they are independent of the background potential $U \geq 0$ and the random potential $V_{\omega} \geq 0$ ), we deduce, similarly to [GK6, Eq. (2.5)], that for all $\omega \in \Omega$ and Borel sets $B$ with $\sup B<\infty$ we have

$$
\mu_{\omega, y}(B):=\operatorname{tr}\left\{T_{y}^{-1} P_{\omega}(B) T_{y}^{-1}\right\} \leq C_{d, v,\left\|V_{\text {per }}^{-}\right\| \text {sup } B}<\infty \quad \text { for all } y \in \mathbb{R}^{d},
$$

and hence

$$
\left\|\chi_{y} P_{\boldsymbol{\omega}}(B)\right\|_{2} \leq C_{d,\left\|V_{\text {per }}^{-}\right\|, \sup B}<\infty \quad \text { for all } y \in \mathbb{R}^{d} .
$$

Note also that $\mu_{\omega}$ and $\mu_{\omega, y}$ are absolutely continuous with respect to each other.

Let $\mathcal{T}_{1}\left(\mathcal{H}_{+}, \mathcal{H}_{-}\right)$be the Banach space of bounded linear operators $A: \mathcal{H}_{+} \rightarrow \mathcal{H}_{-}$ with $T_{-}^{-1} A T_{+}^{-1}$ trace class. Then for all $\omega \in \Omega$ there exists a $\mu_{\omega}$-locally integrable function $\boldsymbol{P}_{\omega}: \mathbb{R} \rightarrow \mathcal{T}_{1}\left(\mathcal{H}_{+}, \mathcal{H}_{-}\right)$, such that

$$
\operatorname{tr}\left\{T_{-}^{-1} \boldsymbol{P}_{\omega}(E) T_{+}^{-1}\right\}=1 \text { for } \mu_{\omega} \text {-a.e. } E,
$$

and, for all Borel sets $B$ with $\sup B<\infty$,

$$
{ }_{-} P_{\omega}(B) l_{+}=\int_{B} \boldsymbol{P}_{\omega}(E) \mathrm{d} \mu_{\omega}(E),
$$

where the integral is the Bochner integral of $\mathcal{T}_{1}\left(\mathcal{H}_{+}, \mathcal{H}_{-}\right)$-valued functions. Note that $\boldsymbol{P}_{\boldsymbol{\omega}}(E)$ is jointly measurable in $(\boldsymbol{\omega}, E)$. (This can be seen from [KIKS, Eq. (46)].) Moreover, we have (e.g., [KIKS, Corollary 3.1])

$$
H_{\omega,-} \boldsymbol{P}_{\omega}(E)=E \boldsymbol{P}_{\omega}(E) \quad \text { for } \mu_{\omega} \text {-a.e. } E \in \mathbb{R},
$$


where $H_{\omega,-}$ is the closure of the operator $H_{\omega}$ in the Hilbert space $\mathcal{H}_{-}$. It follows that

$$
\boldsymbol{P}_{\omega}(E) \mathcal{H}_{+} \subset \widetilde{\Theta}_{\omega}(E) \text { for } \mu_{\omega} \text {-a.e. } E \in \mathbb{R} \text {. }
$$

If for a given Borel set $B$ we have $\left(\mathcal{H}=\iota_{-} \mathcal{H}\right.$ as sets)

$$
\boldsymbol{P}_{\omega}(E) \mathcal{H}_{+} \subset \mathcal{H} \quad \text { for } \mu_{\omega} \text {-a.e } E \in B
$$

it follows from (5.25) that $H_{\omega}$ has pure point spectrum in $B$.

Given $\omega \in \Omega, x \in \mathbb{R}^{d}, E \in \mathbb{R}$, and a scale $L$, we set (cf. [GK6])

$$
\begin{aligned}
\boldsymbol{W}_{\boldsymbol{\omega}, x}(E) & := \begin{cases}\sup _{\substack{\phi \in \mathcal{H}_{+} \\
\boldsymbol{P}_{\omega}(E) \phi \neq 0}} \frac{\left\|\chi_{x} \boldsymbol{P}_{\omega}(E) \phi\right\|}{\left\|T_{x}^{-1} \boldsymbol{P}_{\omega}(E) \phi\right\|} & \text { if } \boldsymbol{P}_{\omega}(E) \neq 0, \\
0 & \text { otherwise, }\end{cases} \\
\boldsymbol{W}_{\boldsymbol{\omega}, x, L}(E) & := \begin{cases}\sup _{\substack{\phi \in \mathcal{H}_{+} \\
\boldsymbol{P}_{\omega}(E) \phi \neq 0 \\
0}} \frac{\left\|\chi_{x, L} \boldsymbol{P}_{\omega}(E) \phi\right\|}{\left\|T_{x}^{-1} \boldsymbol{P}_{\omega}(E) \phi\right\|} & \text { if } \boldsymbol{P}_{\omega}(E) \neq 0,\end{cases}
\end{aligned}
$$

$\boldsymbol{W}_{\boldsymbol{\omega}, x}(E)$ and $\boldsymbol{W}_{\boldsymbol{\omega}, x, L}(E)$ are measurable functions of $(\boldsymbol{\omega}, E)$ for each $x \in \mathbb{R}^{d}$ with

$$
\begin{aligned}
\boldsymbol{W}_{\boldsymbol{\omega}, x}(E) & \leq(5 / 4)^{v / 2}<2^{v / 2}, \\
\boldsymbol{W}_{\boldsymbol{\omega}, x, L}(E) & \leq\left(1+(L+1 / 2)^{2}\right)^{v / 2} \leq 2^{v / 2} L^{v}, \\
\boldsymbol{W}_{\boldsymbol{\omega}, y}(E) & \leq 2^{v} L^{v} \boldsymbol{W}_{\boldsymbol{\omega}, x, L} \quad \text { for } y \in \bar{\Lambda}_{2 L, L}(x) .
\end{aligned}
$$

Moreover, it follows from (5.25) that

$$
\boldsymbol{W}_{\boldsymbol{\omega}, x}(E) \leq W_{\boldsymbol{\omega}, x}(E) \text { and } \boldsymbol{W}_{\boldsymbol{\omega}, x, L}(E) \leq W_{\boldsymbol{\omega}, x, L}(E) \quad \text { for } \mu_{\boldsymbol{\omega}} \text {-a.e. } E \in \mathbb{R} .
$$

Remark 5.5. There is a difference between $\boldsymbol{W}_{\boldsymbol{\omega}, x}(E)$ and $\boldsymbol{W}_{\boldsymbol{\omega}, x, L}(E)$, defined in (5.27) and (5.28), and $W_{\omega, x}(E)$ and $W_{\omega, x, L}(E)$, previously defined in (5.5) and (5.10). The conclusions of the multiscale analysis of Proposition 4.6 will yield bounds on $W_{\omega, x}(E)$ and $W_{\omega, x, L}(E)$ in an energy interval $I$. In view of (5.32), these bounds will hold for $\boldsymbol{W}_{\boldsymbol{\omega}, x}(E)$ and $\boldsymbol{W}_{\boldsymbol{\omega}, x, L}(E)$ for $\mu_{\boldsymbol{\omega}}$-a.e. $E \in I$, yielding (5.26) for $\mu_{\boldsymbol{\omega}}$-a.e. $E \in I$, and hence establishing pure point spectrum in the interval $I$. Note that $\boldsymbol{W}_{\boldsymbol{\omega}, x}(E)$ and $\boldsymbol{W}_{\boldsymbol{\omega}, x, L}(E)$ are measurable functions of $(\omega, E)$ for each $x \in \mathbb{R}^{d}$, but we do not make such a claim for $W_{\omega, x}(E)$ and $W_{\omega, x, L}(E)$.

\subsection{Connection with point spectrum}

Given $E \in \mathbb{R}$, we set

$$
P_{\omega}(E):=\chi_{\{E\}}\left(H_{\omega}\right) \quad \text { and } \quad \mu_{\omega}(E):=\mu_{\omega}(\{E\})=\left\|T^{-1} P_{\omega}(E)\right\|_{2}^{2} .
$$

In particular, $P_{\omega}(E) \neq 0$ if and only if $\mu_{\omega}(E) \neq 0$. 
It follows from (5.23) that

$$
l_{-} P_{\omega}(E) l_{+}=\boldsymbol{P}_{\omega}(E) \mu_{\omega}(E) .
$$

Thus, given $x \in \mathbb{R}^{d}$ and a scale $L$, we have

$$
\begin{gathered}
\left\|\chi_{x} P_{\boldsymbol{\omega}}(E)\right\|_{2} \leq \boldsymbol{W}_{\boldsymbol{\omega}, x}(E)\left\|T_{x}^{-1} P_{\boldsymbol{\omega}}(E)\right\|_{2}=\boldsymbol{W}_{\boldsymbol{\omega}, x}(E) \sqrt{\mu_{\boldsymbol{\omega}, x}(E)} \\
\left\|\chi_{x, L} P_{\boldsymbol{\omega}}(E)\right\|_{2} \leq \boldsymbol{W}_{\boldsymbol{\omega}, x, L}(E)\left\|T_{x}^{-1} P_{\boldsymbol{\omega}}(E)\right\|_{2}=\boldsymbol{W}_{\boldsymbol{\omega}, x, L}(E) \sqrt{\mu_{\boldsymbol{\omega}, x}(E)}
\end{gathered}
$$

If $H_{\omega}$ has pure point spectrum in an interval $I$, it follows from (5.23) and (5.34) that for all bounded Borel functions $f$ we have

$$
f\left(H_{\boldsymbol{\omega}}\right) P_{\boldsymbol{\omega}}(I)=\int_{I} f(E) P_{\boldsymbol{\omega}, x}(E) \mathrm{d} \mu_{\boldsymbol{\omega}, x}(E) \quad \text { for all } x \in \mathbb{R}^{d},
$$

where

$$
P_{\omega, x}(E):= \begin{cases}\left(\mu_{\omega, x}(E)\right)^{-1} P_{\omega}(E) & \text { if } P_{\omega}(E) \neq 0 \\ 0 & \text { otherwise }\end{cases}
$$

\section{From the multiscale analysis to localization}

We will now assume that the conclusions of the multiscale analysis (i.e., of Proposition 4.6) hold for all energies in a bounded open interval $\mathcal{I}$, and prove a theorem that encapsulates localization in the interval $\mathcal{I}$. All forms of localization will be derived from this theorem.

We fix $v>d / 2$, which will be generally omitted from the notation.

Theorem 6.1. Let $H_{\omega}$ be a generalized Anderson Hamiltonian on $\mathrm{L}^{2}\left(\mathbb{R}^{d}\right)$. Consider a bounded open interval $\mathcal{I} \subset \mathbb{R}, m>0, p>0$, and $\varsigma \in] 0,1[$, and assume there is a scale $\mathcal{L}$ such that all scales $L \geq \mathcal{L}$ are $(E, m, \varsigma, p)$-good for all energies $E \in \mathcal{I}$. Set

$$
M=M(m, p):=m / 30^{\widehat{n}+2} \text { where } \widehat{n}=\widehat{n}(p):=\min \left\{n \in \mathbb{N} ; 2^{1 / n}-1<p\right\} .
$$

Fix $\tilde{p} \in] 0, p\left[\right.$, and pick $\vartheta=\beta / 2$, where $\beta=\rho^{n_{1}}$ with $\rho>0$ and $n_{1} \in \mathbb{N}$ such that

$$
(1+p)^{-1}<\rho<1 \text { and }\left(n_{1}+1\right) \beta<p-\tilde{p},
$$

and set, at scale $L$,

$$
\mathcal{I}_{L}:=\left\{E \in \mathcal{I} ; \operatorname{dist}(E, \mathbb{R} \backslash \mathcal{I})>\mathrm{e}^{-M L^{\vartheta}}\right\}
$$

Then, given a sufficiently large scale $L$, for each $x_{0} \in \mathbb{R}^{d}$ there exists an event $\mathcal{U}_{L, x_{0}}$ with the following properties:

(i) We have

$$
\mathcal{U}_{L, x_{0}} \in \mathcal{F}_{\Lambda_{L_{+}}\left(x_{0}\right)} \quad \text { and } \quad \mathbb{P}\left\{\mathcal{U}_{L, x_{0}}\right\} \geq 1-L^{-\tilde{p} d}
$$


(ii) If $\omega \in \mathcal{U}_{L, x_{0}}$ and $E \in \mathcal{I}_{L}$, whenever

$$
W_{\boldsymbol{\omega}, x_{0}}(E)>\mathrm{e}^{-M L^{\vartheta}},
$$

we conclude that

$$
W_{\omega, x_{0}, L}(E) \leq \mathrm{e}^{-M L} .
$$

(iii) If $\omega \in \mathcal{U}_{L, x_{0}}$, we have

$$
W_{\boldsymbol{\omega}, x_{0}}(E) W_{\boldsymbol{\omega}, x_{0}, L}(E) \leq \mathrm{e}^{-\frac{1}{2} M L^{\vartheta}} \quad \text { for all } E \in \mathcal{I}_{L} .
$$

Remark 6.2. If $p \in] 1 / 3,3 / 8[$, as in Theorem 4.1, we have $\widehat{n}=3$.

The proof of this theorem will require several propositions. The scale $\mathcal{L}$ will always be assumed to be sufficiently large; in particular we assume $m \geq \mathcal{L}^{-5 / 2}$. We consider only scales $L \geq \mathcal{L}$. We use the notation $A^{(\mathcal{I})}=A \cap \mathcal{I}$ for $A \subset \mathbb{R}$.

We assume the hypotheses of Theorem 6.1 in the remainder of this section.

\subsection{The first spectral reduction}

Proposition 6.3. Given $b \geq 1$, there exists a constant $K_{d, p, b} \geq 1$ with the following property: Fix $K \geq K_{d, p, b}$. Then, given a sufficiently large scale $L$, for each $x_{0} \in \mathbb{R}^{d}$ there is an event $\mathcal{Q}_{L, x_{0}}$ with

$$
\mathcal{Q}_{L, x_{0}} \in \mathcal{F}_{\Lambda_{L}\left(x_{0}\right)} \text { and } \mathbb{P}\left\{\mathcal{Q}_{L, x_{0}}\right\} \geq 1-L^{-2 b d},
$$

such that for $\omega \in \mathcal{Q}_{L, x_{0}}$, given $E \in \mathcal{I}$ such that

$$
W_{\omega, x_{0}}(E)>\mathrm{e}^{-\widehat{m} \sqrt{L / K}} \quad \text { and } \operatorname{dist}(E, \mathbb{R} \backslash \mathcal{I})>\mathrm{e}^{-\widehat{m} \sqrt{L / K}},
$$

where $\widehat{m}=\widehat{m}(m, p):=30 M$ with $M$ given in (6.1), it follows that

$$
\operatorname{dist}\left(E, \sigma^{(\mathcal{I})}\left(H_{\omega, \Lambda_{L}\left(x_{0}\right)}\right)\right) \leq \mathrm{e}^{-\widehat{m} L} .
$$

The proof of this proposition will rely on several lemmas.

6.1.1. A site percolation model. Given a box $\Lambda_{L^{\prime}}\left(x_{0}\right)$ and a scale $\ell \ll L^{\prime}$, we set $L^{\prime \prime}=$ $L^{\prime}+\ell$, let $\alpha=\alpha_{L^{\prime \prime}, \ell}$ be as in (3.55), and consider the graph

$$
\mathbb{G}=\mathbb{G}_{x_{0}, L^{\prime}, \ell}:=x_{0}+\alpha \ell \mathbb{Z}^{d} \quad \text { with edges } \quad\left\{\left\{r, r^{\prime}\right\} \subset \mathbb{G} ;\left\|r-r^{\prime}\right\|=\alpha \ell\right\} .
$$

Note that for $r, r^{\prime} \in \mathbb{G}$ we have

$$
\left\|r-r^{\prime}\right\|=\alpha \ell \Leftrightarrow r \neq r^{\prime} \text { and } \Lambda_{\ell}(r) \cap \Lambda_{\ell}\left(r^{\prime}\right) \neq \emptyset .
$$

The external boundary of $\Gamma \subset \mathbb{G}$ is defined as

$$
\partial^{+} \Gamma:=\left\{r \in \mathbb{G} \backslash \Gamma ;\left\{r, r^{\prime}\right\} \text { is an edge for some } r^{\prime} \in \Gamma\right\} .
$$


We have \#( $\left(\partial^{+}\{r\}\right)=3^{d}-1$ for all $r \in \mathbb{G}$, i.e., any site is connected by edges to $3^{d}-1$ other sites. We call $y_{0}, y_{1}, \ldots, y_{k} \in \mathbb{G}$ a path if $\left\{y_{j-1}, y_{j}\right\}$ is an edge of $\mathbb{G}$ for $j=1, \ldots, k$; it is a self-avoiding path if the $y_{0}, y_{1}, \ldots, y_{k}$ are distinct.

Given an energy $E \in \mathcal{I}$, we consider the following site percolation model on the graph $\mathbb{G}$ : every site $r \in \mathbb{G}_{\Lambda_{L^{\prime \prime}}\left(x_{0}\right)}^{(\ell)}=\mathbb{G} \cap \Lambda_{L^{\prime \prime}}\left(x_{0}\right)$ (cf. (3.48)) is bad with probability one; a site $r \in \mathbb{G} \backslash \mathbb{G}_{\Lambda_{L^{\prime \prime}}\left(x_{0}\right)}^{(\ell)}$ is good if the box $\Lambda_{\ell}(r)$ is $(\omega, E, m, \varsigma, p)$-good and bad otherwise. We let $\mathbb{A}_{E}=\mathbb{A}_{E}(\boldsymbol{\omega})=\mathbb{A}_{E, x_{0}, L^{\prime}, \ell}(\boldsymbol{\omega})$ denote the cluster of bad sites containing $\mathbb{G}_{\Lambda_{L^{\prime \prime}}\left(x_{0}\right)}^{(\ell)}$ (i.e., the connected component of the subgraph of bad sites containing $\mathbb{G}_{\Lambda_{L^{\prime \prime}}\left(x_{0}\right)}^{(\ell)}$ ).

We now take scales $\ell, \widetilde{L}$ with $\ell \ll L^{\prime}$ and $100^{d} \ell \leq \widetilde{L}$. Given an energy $E \in \mathbb{R}$, we consider the event

$$
\mathcal{Y}_{x_{0}, L^{\prime}, \ell, \widetilde{L}}^{(E)}:= \begin{cases}\left\{\mathbb{A}_{E} \subset \Lambda_{L^{\prime}+\widetilde{L}-3 \ell}\left(x_{0}\right)\right\} & \text { if } E \in \mathcal{I} \\ \Omega & \text { if } E \notin \mathcal{I}\end{cases}
$$

Note that $\mathcal{Y}_{x_{0}, L^{\prime}, \ell, \widetilde{L}}^{(E)} \in \mathcal{F}_{\Lambda_{L^{\prime}+\widetilde{L}, L^{\prime}}\left(x_{0}\right)}$ for all $E \in \mathbb{R}$, and it follows from (3.6) that $\mathcal{Y}_{x_{0}, L^{\prime}, \ell, \tilde{L}}^{(E)}$ is jointly measurable in $\left(E, \omega_{\Lambda_{L^{\prime}+\tilde{L}, L^{\prime}}\left(x_{0}\right)}\right)$.

Lemma 6.4. For all $E \in \mathcal{I}$ we have

$$
\mathbb{P}\left\{\mathcal{Y}_{x_{0}, L^{\prime}, \ell, \tilde{L}}^{(E)}\right\} \geq 1-\left(4 L^{\prime} / \ell\right)^{d-1} 3^{[\widetilde{L} / 2 \ell] d} \ell^{-c_{d} p \widetilde{L} / \ell} .
$$

In particular, if $L^{\prime}=\widetilde{L}=L / 2$ and $\ell=\sqrt{L}$, we get

$$
\mathbb{P}\left\{\mathcal{Y}_{x_{0}, L / 2, \sqrt{L}, L / 2}^{(E)}\right\} \geq 1-L^{-c_{d, p} \sqrt{L}} .
$$

Proof. Fix $E \in \mathcal{I}$, and suppose $\mathbb{A}_{E} \not \subset \Lambda_{L^{\prime}+\widetilde{L}-3 \ell}\left(x_{0}\right)$. Then there exists a self-avoiding path $y_{0}, y_{1}, \ldots, y_{k}$ in $\mathbb{G}$ such that $\left\|y_{0}-x_{0}\right\|=L^{\prime} / 2, y_{1}, y_{2}, \ldots, y_{k} \notin \mathbb{G}_{\Lambda_{L^{\prime \prime}}\left(x_{0}\right)}^{(\ell)}$,

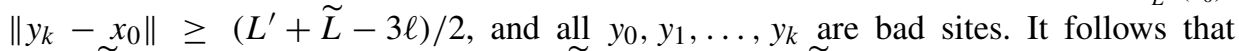
$\left(L^{\prime}+\widetilde{L}-3 \ell\right) / 2 \leq L^{\prime} / 2+k \alpha \ell$, so $k \geq(\widetilde{L}-3 \ell) / 2 \alpha \ell \geq[\widetilde{L} / 2 \ell]$. We thus conclude that if $\mathbb{A}_{E} \not \subset \Lambda_{L^{\prime}+\widetilde{L}-3 \ell}\left(x_{0}\right)$ we can find a self-avoiding path $y_{0}, y_{1}, \ldots, y_{[\widetilde{L} / 2 \ell]}$ of bad sites with $\left\|y_{0}-x_{0}\right\|=L^{\prime} / 2$ and $y_{1}, y_{2}, \ldots, y_{[\tilde{L} / 2 \ell]} \notin \mathbb{G}_{\Lambda_{L^{\prime \prime}}\left(x_{0}\right)}^{(\ell)}$. The number of such self-avoiding paths is bounded by $\left(4 L^{\prime} / \ell\right)^{d-1} 3^{[\widetilde{L} / 2 \ell] d}$. Since sites $y, y^{\prime} \notin \mathbb{G}_{\Lambda_{L^{\prime \prime}}\left(x_{0}\right)}^{(\ell)}$ are independent unless $\left\|y-y^{\prime}\right\| \leq \alpha \ell$, such a self-avoiding path must contain at least $\left[3^{-d}[\tilde{L} / 2 \ell]\right] \geq c_{d}^{\prime} \tilde{L} / \ell$ independent sites, and hence its probability of having only bad sites is $\leq \ell^{-c_{d}^{\prime} p d \widetilde{L} / \ell}$. Thus

$$
\mathbb{P}\left\{\mathbb{A}_{E} \not \subset \Lambda_{L^{\prime}+\widetilde{L}-3 \ell}\left(x_{0}\right)\right\} \leq\left(4 L^{\prime} / \ell\right)^{d-1} 3^{[\widetilde{L} / 2 \ell] d} \ell^{-c_{d}^{\prime} p d \tilde{L} / \ell} .
$$

Given $\Gamma \subset \mathbb{G}$ and $0 \leq \varepsilon_{1}<\varepsilon_{2}$, we set

$$
\widehat{\Gamma}:=\bigcup_{x \in \Gamma} \Lambda_{\ell}(x), \quad \partial^{\left(\varepsilon_{1}, \varepsilon_{2}\right)} \widehat{\Gamma}:=\left\{x \in \mathbb{R}^{d} ; \varepsilon_{1}<\operatorname{dist}(x, \widehat{\Gamma})<\varepsilon_{2}\right\} .
$$

Note that $\widehat{\Gamma}$ is a connected subset of $\mathbb{R}^{d}$ if $\Gamma$ is a connected subset of $\mathbb{G}$. 
Lemma 6.5. Let $E \in \mathcal{I}$ and $\omega \in \mathcal{Y}_{x_{0}, L^{\prime}, \ell, \widetilde{L}^{\prime}}^{(E)}$ Then $\left(\mathbb{A}_{E}=\mathbb{A}_{E}(\boldsymbol{\omega})\right)$ :

(i) For all $r \in \partial^{+} \mathbb{A}_{E}$ we have $\Lambda_{\ell}(r) \subset \Lambda_{L^{\prime}+\widetilde{L}}\left(x_{0}\right)$ and the box $\Lambda_{\ell}(r)$ is $(\omega, E, m, \varsigma, p)$ good.

(ii) There exists a function $\phi=\phi_{\omega, E} \in C_{\mathrm{c}}^{2}\left(\mathbb{R}^{d}\right)$, with $0 \leq \phi \leq 1$, such that

$$
\begin{aligned}
& \phi \equiv 1 \quad \text { on } \widehat{\mathbb{A}_{E}}, \\
& \phi \equiv 0 \quad \text { on } \mathbb{R}^{d} \backslash \Lambda_{L^{\prime}+\widetilde{L}-\ell}\left(x_{0}\right), \\
& \operatorname{supp} \nabla \phi \subset \partial^{(4,8)} \widehat{\mathbb{A}_{E}}, \\
& |\nabla \phi|,|\Delta \phi| \leq C_{d}, \quad \text { the constant } C_{d} \text { depending only on } d,
\end{aligned}
$$

and for all $x \in \mathbb{R}^{d}$ with $\Lambda_{1 / 2}(x) \cap \operatorname{supp} \nabla \phi \neq \emptyset$ there exists $r(x) \in \partial^{+} \mathbb{A}_{E}$ such that $\Lambda_{\ell / 5}(x) \subset \Lambda_{\ell}(r(x))$.

Proof. Since $\omega \in \mathcal{Y}_{x_{0}, L^{\prime}, \ell, \tilde{L}}^{(E)}$, we have $\widehat{\mathbb{A}_{E}} \subset \Lambda_{L^{\prime}+\tilde{L}-2 \ell}\left(x_{0}\right)$. (i) follows from the definition of $\widehat{\mathbb{A}_{E}}$. To prove (ii), let $\psi$ be the characteristic function of the set $\left\{x \in \mathbb{R}^{d} ; \operatorname{dist}\left(x, \widehat{\mathbb{A}_{E}}\right)\right.$ $\leq 6\}$. Pick a non-negative function $\eta \in C^{2}\left(\mathbb{R}^{d}\right)$ with compact support in $\Lambda_{1}(0)$, $\int_{\mathbb{R}^{d}} \eta(x) \mathrm{d} x=1$, and $|\nabla \eta|,|\Delta \eta| \leq C_{d}^{\prime}$. Then $\phi=\eta * \psi$ has all the desired properties.

Let $x \in \mathbb{R}^{d}$ with $\Lambda_{1 / 2}(x) \cap \operatorname{supp} \nabla \phi \neq \emptyset$. Then, in view of (3.54), there exists $r(x) \in \partial^{+} \mathbb{A}_{E}$ with $\Lambda_{\ell / 5}(x) \subset \Lambda_{\ell}(r(x))$. Since $x \in \partial^{(3,9)} \widehat{\mathbb{A}_{E}} \cap \Lambda_{\ell}(r)$ for some $r \in \mathbb{G}$ implies $r \in \partial^{+} \mathbb{A}_{E}$, we conclude that $r(x) \in \partial^{+} \mathbb{A}_{E}$.

\subsubsection{The energy trap}

Lemma 6.6. Given a sufficiently large scale L, for each $x_{0} \in \mathbb{R}^{d}$ there exists an event $\mathcal{T}_{L, x_{0}}$ with

$$
\mathcal{T}_{L, x_{0}} \in \mathcal{F}_{\Lambda_{L, L / 2}\left(x_{0}\right)} \quad \text { and } \quad \mathbb{P}\left\{\mathcal{T}_{L, x_{0}}\right\} \geq 1-L^{-c_{d, p, m,|\mathcal{I}|} \sqrt{L}}
$$

such that for $\omega \in \mathcal{T}_{L, x_{0}}$ we have

$$
W_{\boldsymbol{\omega}, x_{0}}(E) \operatorname{dist}\left(E, \sigma\left(H_{\left.\boldsymbol{\omega}, \Lambda_{L}\left(x_{0}\right)\right)} \leq \mathrm{e}^{-\frac{m}{15} \sqrt{L}} \quad \text { for all } E \in \mathcal{I}\right.\right.
$$

In particular, for $\omega \in \mathcal{T}_{L, x_{0}}$ and $E \in \mathcal{I}$,

$$
W_{\boldsymbol{\omega}, x_{0}}(E)>\mathrm{e}^{-\frac{m}{30} \sqrt{L}} \Rightarrow \operatorname{dist}\left(E, \sigma\left(H_{\boldsymbol{\omega}, \Lambda_{L}\left(x_{0}\right)}\right)\right) \leq \mathrm{e}^{-\frac{m}{30} \sqrt{L}} .
$$

Proof. Fix a scale $L$ and $x_{0} \in \mathbb{R}^{d}$. Since $\mathcal{I}$ is a bounded interval, we can find $\left\{E_{j}\right\}_{j=1, \ldots, J}$ $\subset \mathcal{I}$ such that

$$
\mathcal{I} \subset \bigcup_{j=1}^{J}\left[E_{j}-\mathrm{e}^{-2 m \sqrt{L}}, E_{j}+\mathrm{e}^{-2 m \sqrt{L}}\right] \quad \text { and } \quad J \leq \mathrm{e}^{2 m \sqrt{L}}|\mathcal{I}|
$$


We set

$$
\mathcal{T}_{L, x_{0}}=\bigcap_{j=1}^{J} \mathcal{Y}_{x_{0}, L / 2, \sqrt{L}, L / 2}^{\left(E_{j}\right)} .
$$

The estimate (6.23) follows immediately from (6.16).

Let $\omega \in \mathcal{T}_{L, x_{0}}$ and $E \in \mathcal{I}$ with $\Theta_{\omega}(E) \neq \varnothing$. Pick $j \in\{1, \ldots, J\}$ such that we have $E \in\left[E_{j}-\mathrm{e}^{-2 m \sqrt{L}}, E_{j}+\mathrm{e}^{-2 m \sqrt{L}}\right]$, write $\mathbb{A}_{E_{j}}=\mathbb{A}_{E_{j}}(\boldsymbol{\omega})$, and let $\phi=\phi_{\boldsymbol{\omega}, E_{j}}$ be the function given in Lemma 6.5. Let $\psi \in \Theta_{\omega}(E)$, a generalized eigenfunction. Then $\phi \psi \in \mathcal{D}\left(H_{\omega, \Lambda}\right)$ and we have (2.23), where $\Lambda=\Lambda_{L}\left(x_{0}\right)$. It follows that, for $\mathcal{L}$ sufficiently large,

$$
\begin{aligned}
& \left\|\left(H_{\omega, \Lambda}-E\right) \phi \psi\right\|^{2}=\left\|W_{\Lambda}(\phi) \psi\right\|^{2}=\sum_{\substack{x \in x_{0}+\frac{1}{2} \mathbb{Z}^{d} \\
\Lambda_{1 / 2}(x) \cap \operatorname{supp} \nabla \phi \neq \emptyset}}\left\|\chi_{\Lambda_{1 / 2}(x)} W_{\Lambda}(\phi) \psi\right\|^{2} \\
& \leq C_{d, \mathcal{I}, V_{\text {per }}} \sum_{\substack{x \in x_{0}+\frac{1}{2} \mathbb{Z}^{d} \\
\Lambda_{1 / 2}(x) \cap \operatorname{supp} \nabla \phi \neq \emptyset}}\left\|\chi_{x} \psi\right\|^{2} \leq C_{d, \mathcal{I}, V_{\text {per }}}^{2} \mathrm{e}^{-\frac{2 m^{\prime}}{11} \sqrt{L}} \sum_{\substack{x \in x_{0}+\frac{1}{2} \mathbb{Z}^{d} \\
\Lambda_{1 / 2}(x) \cap \operatorname{supp} \nabla \phi \neq \emptyset}}\|\psi\|_{\Lambda_{\sqrt{L}}(r(x))}^{2} \\
& \leq C_{d, \mathcal{I}, V_{\text {per }}}^{\prime} L^{d} \mathrm{e}^{-\frac{2 m^{\prime}}{11} \sqrt{L}}\|\psi\|_{\Lambda_{L, \frac{L}{2}}\left(x_{0}\right)}^{2} \leq \mathrm{e}^{-\frac{2 m}{15} \sqrt{L}}\left\|T_{x_{0}}^{-1} \psi\right\|^{2}
\end{aligned}
$$

where we used (2.24) and (6.22), applied the interior estimate given in (2.43) as in the derivation of (2.44), used Lemma 6.5 with $\ell=\sqrt{L}\left(r(x) \in \partial^{+} \mathbb{A}_{E_{j}}\right.$ is given in the lemma), applied Lemma 3.9(iii), using (3.22) with $m^{\prime}$ as in (3.17) taking $\ell=\sqrt{L}$, and then used (3.17) to write the final estimate in terms of $m$. Since it follows from (6.19) that $\|\phi \psi\| \geq\|\psi\|_{\Lambda_{L / 2}\left(x_{0}\right)} \geq\left\|\chi_{x_{0}} \psi\right\|$, we conclude that

$$
\operatorname{dist}\left(E, \sigma\left(H_{\omega, \Lambda_{L}\left(x_{0}\right)}\right)\right) \leq \frac{\left\|\left(H_{\omega, \Lambda}-E\right) \phi \psi\right\|}{\|\phi \psi\|} \leq \mathrm{e}^{-\frac{m}{15} \sqrt{L}} \frac{\left\|T_{x_{0}}^{-1} \psi\right\|}{\left\|\chi_{x_{0}} \psi\right\|}
$$

The desired (6.24) now follows using (5.5), and it yields (6.25) .

6.1.3. The energy bootstrap. We fix $b \geq 1$, let $\widehat{n}=\widehat{n}(p)$ be as in (6.1), and set

$$
\left.\left.\eta=\eta(p):=2^{1 / \widehat{n}}-1<p, \quad \text { so } \quad \eta \in\right] 0,1\right] \quad \text { and } \quad(1+\eta)^{\widehat{n}}=2 .
$$

We now fix a scale $L$, let $\ell_{0}=\sqrt{L}$, and set $\ell_{k}=\ell_{k-1}^{1+\eta}$ for $k=1, \ldots, \widehat{n}$, so $\ell_{\widehat{n}}=L$ by (6.30). We take $J \in \mathbb{N}$, to be determined later, and let $L_{0}=L, L_{k}=L_{k-1}+2 J \ell_{k}$ for $k=1, \ldots, \widehat{n}$. We have

$$
L_{\widehat{n}}=L+2 J \sum_{k=1}^{\widehat{n}} \ell_{k} \leq(1+2 J \widehat{n}) L
$$

Given $x_{0} \in \mathbb{R}^{d}$ and $E \in \mathcal{I}$, we consider the events $\widetilde{\mathcal{Y}}_{x_{0}, L_{k-1}, \ell_{k}, 2 J \ell_{k}}^{(E)}, k=1, \ldots, \widehat{n}$, defined similarly to the event in (6.14), but with a modified site percolation model: a 
site $r$ is now either pgood or pbad according to whether the corresponding box $\Lambda_{\ell_{k}}$ is $(\omega, E, m, \varsigma, \eta)$-pgood or not (see Definition 3.15), and the set $\widetilde{\mathbb{A}}_{E}(\omega)$, defined similarly to $\mathbb{A}_{E}(\boldsymbol{\omega})$, is now a cluster of bad sites. Requiring $2 J \geq 100^{d}$, Lemma 6.4 still applies, with $\widehat{p}=\widehat{p}(p):=p-\eta /(2(1+\eta))$ substituted for $p$ in view of Lemma 3.17, yielding for all $k=1, \ldots, \widehat{n}$ the estimate

$$
\begin{aligned}
\mathbb{P}\left\{\tilde{\mathcal{Y}}_{x_{0}, L_{k-1}, \ell_{k}, 2 J \ell_{k}}^{(E)}\right\} & \geq 1-\left(4 L_{k-1} / \ell_{k}\right)^{d-1} 3^{J d} \ell_{k}^{-2 c_{d} \widehat{p} J} \geq 1-\left(4 L_{\widehat{n}} / \ell_{0}\right)^{d-1} 3^{J d} \ell_{0}^{-2 c_{d} \widehat{p}^{J}} \\
& \geq 1-\left(4(1+2 J \widehat{n}) L^{1 / 2}\right)^{d-1} 3^{J d} L^{-c_{d} \widehat{p} J} \geq 1-L^{-6 b d}
\end{aligned}
$$

provided $J \geq C_{d, p, b}$. We fix $J_{d, p, b}:=\left[\max \left\{C_{d, p, b}, 100^{d} / 2\right\}\right]+1$ so if $J \geq J_{d, p, b}$ the estimate (6.32) holds for all $k=1, \ldots, \widehat{n}$.

For each $k=0,1, \ldots, \widehat{n}-1$ the finite volume operator $H_{\Lambda_{L_{k}}\left(x_{0}\right), \omega}$, which depends only on $\omega_{\Lambda_{L_{k}}\left(x_{0}\right)}$, is a non-negative self-adjoint operator with discrete spectrum. We let $\left\{E_{j}^{(k)}\left(\boldsymbol{\omega}_{\Lambda_{L_{k}}\left(x_{0}\right)}\right)\right\}_{j \in \mathbb{N}}$ be the enumeration of these eigenvalues given by the min-max principle, as in (4.64). Each $E_{j}^{(k)}=E_{j}^{(k)}\left(\omega_{\Lambda_{L_{k}}\left(x_{0}\right)}\right)$ is a continuous function of $\omega_{\Lambda_{L_{k}}\left(x_{0}\right)}$. We define events

$$
\mathcal{Z}_{k}=\mathcal{Z}_{\Lambda_{L_{k}}\left(x_{0}\right)}:=\bigcap_{j \in \mathbb{N}} \tilde{\mathcal{Y}}_{x_{0}, L_{k-1}, \ell_{k}, 2 J \ell_{k}}^{(k-1)} \in \mathcal{F}_{\Lambda_{L_{k}}\left(x_{0}\right)} \quad \text { for } k=1, \ldots, \widehat{n} .
$$

Note that $\mathcal{Z}_{k} \in \mathcal{F}_{\Lambda_{L_{k}}\left(x_{0}\right)}$ since the event $\tilde{\mathcal{Y}}_{x_{0}, L_{k-1}, \ell_{k}, 2 J \ell_{k}}^{(E)}$ is jointly measurable in $\left(E, \omega_{\Lambda_{L_{k}, L_{k-1}}\left(x_{0}\right)}\right)$ and each $E_{j}^{(k-1)}$ is a measurable function of $\boldsymbol{\omega}_{\Lambda_{L_{k-1}}\left(x_{0}\right)}$. Since general estimates yield (cf. [GK5, Eq. (A.7)])

$$
\operatorname{tr}\left\{\chi_{\mathcal{I}}\left(H_{\Lambda_{L}\left(x_{0}\right), \omega}\right)\right\} \leq C_{d, V_{\text {per }}, \sup \mathcal{I}} L^{d} \quad \text { for all } L \geq 10,
$$

it follows from (6.14) and (6.32) that

$$
\mathbb{P}\left\{\mathcal{Z}_{k}\right\} \geq 1-L^{-4 b d} \quad \text { for } k=1, \ldots, \widehat{n} .
$$

Lemma 6.7. Given a sufficiently large scale $L$, for each $x_{0} \in \mathbb{R}^{d}$ there exists an event $\mathcal{Z}_{L, x_{0}}$ with

$$
\mathcal{Z}_{L, x_{0}} \in \mathcal{F}_{\Lambda_{L_{\widehat{n}}}\left(x_{0}\right)} \quad \text { and } \quad \mathbb{P}\left\{\mathcal{Z}_{L, x_{0}}\right\} \geq 1-\widehat{n} L^{-4 b d} \geq 1-L^{-3 b d}
$$

such that for all $\omega \in \mathcal{Z}_{L, x_{0}}$, if $E \in \mathcal{I}$ satisfies

$$
\begin{aligned}
& \operatorname{dist}\left(E, \sigma^{(\mathcal{I})}\left(H_{\omega, \Lambda_{L}\left(x_{0}\right)}\right)\right) \leq \mathrm{e}^{-\frac{m}{30} \sqrt{L}}, \\
& \operatorname{dist}(E, \mathbb{R} \backslash \mathcal{I})>\mathrm{e}^{-\widehat{m} \sqrt{L}}, \\
& W_{\boldsymbol{\omega}, x_{0}}(E)>\mathrm{e}^{-\widehat{m} \sqrt{L}},
\end{aligned}
$$

where $\widehat{m}:=m / 30^{\widehat{n}+1}=30 M($ see $(6.1))$, it follows that

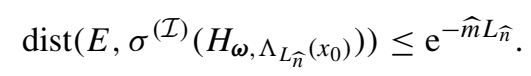


Proof. Given $L$ and $x_{0}$, we set

$$
\mathcal{Z}_{L, x_{0}}:=\bigcap_{k=1}^{\widehat{n}} \mathcal{Z}_{k}
$$

so (6.36) follows immediately from (6.35).

Let $\tilde{m}=m / 30$. Given $\omega \in \mathcal{Z}_{L, x_{0}}$ and $E \in \mathcal{I}$ satisfying (6.37) and $W_{\omega, x_{0}}(E)>0$, we pick $E^{\prime} \in \sigma^{(\mathcal{I})}\left(H_{\omega, \Lambda_{L}\left(x_{0}\right)}\right)$ such that $\left|E-E^{\prime}\right| \leq \mathrm{e}^{-\widetilde{m} \sqrt{L}}$ and $\psi \in \Theta_{\omega}(E)$. We have $\omega \in \tilde{\mathcal{Y}}_{x_{0}, L, \ell_{1}, 2 J \ell_{1}}^{\left(E^{\prime}\right)}$, so we let $\phi=\phi_{\omega, E^{\prime}}$ be the function given in Lemma 6.5. Note that Lemma 6.5 applies as stated for the modified site percolation model, the only modification being that a box $\Lambda_{\ell_{1}}(r)$ with $r \in \partial^{+} \widetilde{\mathbb{A}}_{E^{\prime}}(\boldsymbol{\omega})$ is now $\left(\boldsymbol{\omega}, E^{\prime}, m, \varsigma, \eta\right)$-pgood, and hence, using Lemma 3.16, $\Lambda_{\ell_{1}}(r)$ is $(\omega, E, \widetilde{\widetilde{m}}, \varsigma)$-good, where

$$
\widetilde{\tilde{m}}=\tilde{m}\left(1-C_{d, p, m} \ell_{1}^{-\min \{s, \eta\} /(1+\eta)}\right) .
$$

Proceeding as in (6.28) and (6.29), we get (for $L$ large)

$$
\frac{\left\|\left(H_{\omega, \Lambda_{L}}\left(x_{0}\right)-E\right) \phi \psi\right\|}{\|\phi \psi\|} \leq \mathrm{e}^{-\frac{\tilde{\tilde{m}}}{13} \ell_{1}} \frac{\left\|T_{x_{0}}^{-1} \psi\right\|}{\left\|\chi_{x_{0}} \psi\right\|} \leq \mathrm{e}^{-\frac{\tilde{\tilde{r}}}{15} \ell_{1}} \frac{\left\|T_{x_{0}}^{-1} \psi\right\|}{\left\|\chi_{x_{0}} \psi\right\|},
$$

the generalized eigenfunction $\psi$ being arbitrary, so we conclude that

$$
\operatorname{dist}\left(E, \sigma\left(H_{\left.\omega, \Lambda_{L_{1}}\left(x_{0}\right)\right)}\right) \leq \mathrm{e}^{-\frac{\tilde{m}}{15} \ell_{1}}\left(W_{\omega, x_{0}}(E)\right)^{-1} .\right.
$$

Since it follows from (6.39) that

$$
W_{\boldsymbol{\omega}, x_{0}}(E)>\mathrm{e}^{-\frac{\tilde{m}}{30} \ell_{1}},
$$

we get, using also (6.38),

$$
\operatorname{dist}\left(E, \sigma^{(\mathcal{I})}\left(H_{\left.\omega, \Lambda_{L_{1}}\left(x_{0}\right)\right)}\right) \leq \mathrm{e}^{-\frac{\tilde{m}}{30} \ell_{1}} .\right.
$$

Repeating the argument $\widehat{n}-1$ times we get (6.40).

6.1.4. Completing the proof of Proposition 6.3. Given a scale $L$, let $\breve{L}$ be the unique scale such that $\breve{L}_{\widehat{n}}=L$ (see (6.31)). We take $J \geq J_{d, p, b}$, so $K=1+2 J \widehat{n} \geq K_{d, p, b}:=$ $1+2 J_{d, p, b} \widehat{n}$, and hence $\breve{L} \geq L / K$. Recalling Lemmas 6.6 and 6.7, we let

$$
\mathcal{Q}_{L, x_{0}}=\mathcal{T}_{\breve{L}, x_{0}} \cap \mathcal{Z}_{\breve{L}, x_{0}} \in \mathcal{F}_{\Lambda_{\breve{L}, \breve{L} / 2}\left(x_{0}\right)} \cap \mathcal{F}_{\Lambda_{\breve{L}_{\widehat{n}}}\left(x_{0}\right)} \subset \mathcal{F}_{\Lambda_{L}\left(x_{0}\right)},
$$

SO

$$
\mathbb{P}\left\{\mathcal{Q}_{L, x_{0}}\right\} \geq 1-\breve{L}^{-c_{d, p, m,|\mathcal{I}|} \sqrt{\check{L}}}-\breve{L}^{-3 d} \geq 1-L^{-2 b d} .
$$

Let $\omega \in \mathcal{Q}_{L, x_{0}}$ and $E \in \mathcal{I}$ satisfying (6.9). It follows that

$$
W_{\boldsymbol{\omega}, x_{0}}(E)>\mathrm{e}^{-\frac{m}{30} \sqrt{\breve{L}}} \text { and } \operatorname{dist}(E, \mathbb{R} \backslash \mathcal{I})>\mathrm{e}^{-\frac{m}{30} \sqrt{\check{L}}},
$$

so we conclude from Lemma 6.6 that

$$
\operatorname{dist}\left(E, \sigma^{(\mathcal{I})}\left(H_{\left.\omega, \Lambda_{\check{L}}\left(x_{0}\right)\right)}\right) \leq \mathrm{e}^{-\frac{m}{30} \sqrt{\breve{L}}} .\right.
$$

Since (6.50) is just (6.37) at scale $\breve{L}$, and (6.9) implies (6.38) and (6.39) at scale $\breve{L}$, Lemma 6.7 now yields (6.40) for the scale $\breve{L}$, which is the desired (6.10). 


\subsection{The second spectral reduction}

If $p \leq 1$ we need a second spectral reduction.

Given a scale $L$, we set $L_{n}=L^{\rho^{n}}$ for $n=0,1, \ldots, n_{1}\left(\right.$ note $\left.L_{0}=L, L_{n_{1}}=L^{\beta}\right)$, where $\rho, n_{1}, \beta$ are as in Theorem 6.1.

Definition 6.8. The reduced spectrum of the operator $H_{\omega}$ in the box $\Lambda_{L}\left(x_{0}\right)$, in the energy interval $\mathcal{I}$, is given by

$$
\sigma^{(\mathcal{I}, \text { red })}\left(H_{\boldsymbol{\omega}, \Lambda_{L}\left(x_{0}\right)}\right):=\left\{E \in \sigma^{(\mathcal{I})}\left(H_{\boldsymbol{\omega}, \Lambda_{L}\left(x_{0}\right)}\right) ; \operatorname{dist}\left(E, \sigma^{(\mathcal{I})}\left(H_{\omega, \Lambda_{L_{n}}\left(x_{0}\right)}\right)\right) \leq 2 \mathrm{e}^{-\widehat{m} L_{n}},\right.
$$

where $\widehat{m}$ is given in (6.1).

Note that the set $\left\{(E, \omega) ; E \in \sigma^{(\mathcal{I}, \text { red })}\left(H_{\boldsymbol{\omega}, \Lambda_{L}\left(x_{0}\right)}\right)\right\}$ is jointly measurable in $\left(E, \omega_{\Lambda_{L}\left(x_{0}\right)}\right)$.

Proposition 6.9. Let $b \geq 1$ and fix $K \geq K_{d, p, b}$, where $K_{d, p, b}$ is the constant of Proposition 6.3. Given a sufficiently large scale $L$, for each $x_{0} \in \mathbb{R}^{d}$ there exists an event $\mathcal{X}_{L, x_{0}}$, with

$$
\mathcal{X}_{L, x_{0}} \in \mathcal{F}_{\Lambda_{L}\left(x_{0}\right)} \quad \text { and } \quad \mathbb{P}\left\{\mathcal{X}_{L, x_{0}}\right\} \geq 1-L^{-b \beta d},
$$

such that for all $\omega \in \mathcal{X}_{L, x_{0}}$ :

(i) If $E \in \mathcal{I}$ satisfies

$$
W_{\boldsymbol{\omega}, x_{0}}(E)>\mathrm{e}^{-\widehat{m} \sqrt{L^{\beta} / K}} \text { and } \operatorname{dist}(E, \mathbb{R} \backslash \mathcal{I})>\mathrm{e}^{-\widehat{m} \sqrt{L^{\beta} / K}},
$$

it follows that

$$
\operatorname{dist}\left(E, \sigma^{(\mathcal{I}, \text { red })}\left(H_{\omega, \Lambda_{L}\left(x_{0}\right)}\right)\right) \leq \mathrm{e}^{-\widehat{m} L}
$$

(ii) We have

$$
\# \sigma^{(\mathcal{I}, \text { red })}\left(H_{\omega, \Lambda_{L}\left(x_{0}\right)}\right) \leq C_{d, V_{\mathrm{per}}, \mathcal{I}, p, \rho, n_{1}} L^{\left(n_{1}+1\right) \beta d} .
$$

The proof will use several lemmas.

Lemma 6.10. Given a sufficiently large scale $L$ and $x_{0} \in \mathbb{R}^{d}$, consider the event

$$
\widetilde{\mathcal{Q}}_{L, x_{0}}:=\bigcap_{n=0}^{n_{1}} \mathcal{Q}_{L_{n}, x_{0}} \in \mathcal{F}_{\Lambda_{L}\left(x_{0}\right)},
$$

where $\mathcal{Q}_{L^{\prime}, x_{0}}$ is the event given in Proposition 6.3 at scale $L^{\prime}$. Then

$$
\mathbb{P}\left\{\widetilde{\mathcal{Q}}_{L, x_{0}}\right\}>1-\left(n_{1}+1\right) L^{-2 b \beta d} .
$$

Moreover, if $\omega \in \widetilde{\mathcal{Q}}_{L, x_{0}}$, we have (6.54) for any $E \in \mathcal{I}$ satisfying (6.53). 
Proof. The estimate (6.57) follows immediately from (6.56), (6.8), and (6.2). The second part of the lemma is an immediate consequence of Proposition 6.3.

Given scales $L^{\prime}<L$ with $L^{\rho}<\left(L-L^{\prime}\right) / 7$ and $x_{0} \in \mathbb{R}$, we consider the annulus $\Lambda_{L, L^{\prime}}=\Lambda_{L, L^{\prime}}\left(x_{0}\right)$. We let $\mathcal{R}_{n}=\left\{\Lambda_{L_{n}}(r)\right\}_{r \in R_{n}}$ denote the standard $L_{n}$-covering of the annulus $\Lambda_{L, L^{\prime}}$ for $n=1, \ldots, n_{1}$ (see Section 3.3.2). Given $K_{2} \in \mathbb{N}$ (to be chosen later), we set

$$
\mathcal{S}_{\Lambda_{L, L^{\prime}}}:=\left\{\bigcup_{r \in R_{n_{1}}^{\prime}} \Lambda_{3 L_{n_{1}}}(r) ; R_{n_{1}}^{\prime} \subset R_{n_{1}} \text { with \# } R_{n_{1}}^{\prime} \leq K_{2}\right\} .
$$

Similarly to Definition 4.10, the annulus $\Lambda_{L, L^{\prime}}$ is said to be $\left(\omega, E, K_{2}\right)$-notsobad if there exists an $\left(\omega, L, L^{\prime}, E\right)$-singular set $\Theta \in \mathcal{S}_{\Lambda_{L, L^{\prime}}}$ : for all $x \in \Lambda_{L, L^{\prime}} \backslash \Theta$ there is an $(\omega, E, m, \varsigma)$-good box $\Lambda_{L_{n}}(r) \in \mathcal{R}_{n}$, for some $n \in\left\{1, \ldots, n_{1}\right\}$, with $\Lambda_{L_{n} / 5}(x) \cap \Lambda_{L, L^{\prime}} \subset$ $\Lambda_{L_{n}}(r)$. An event $\mathcal{N}$ is $\left(\Lambda_{L, L^{\prime}}, E, K_{2}\right)$-notsobad if $\mathcal{N} \in \mathcal{F}_{\Lambda_{L, L^{\prime}}}$ and the annulus $\Lambda_{L, L^{\prime}}$ is $\left(\omega, E, K_{2}\right)$-notsobad for all $\omega \in \mathcal{N}$. We have the analogue of Lemma 4.11: If $K_{2} \geq$ $\widehat{K}_{2}=\widehat{K}_{2}(d, p, b)$, and $L \geq \widehat{L}=\widehat{L}\left(d, p, b, K_{2}\right)$, then for all $E \in \mathcal{I}$ there exists a $\left(\Lambda_{L, L^{\prime}}, E, K_{2}\right)$-notsobad event $\mathcal{N}_{\Lambda_{L, L^{\prime}}}^{(E)}$ with

$$
\mathbb{P}\left\{\mathcal{N}_{\Lambda_{L, L^{\prime}}}^{(E)}\right\}>1-L^{-5 b d}
$$

(The proof of Lemma 4.11 applies since $\rho>(1+p)^{-1}$.) We fix $K_{2}=\left[\widehat{K}_{2}\right]+1$, so (6.59)

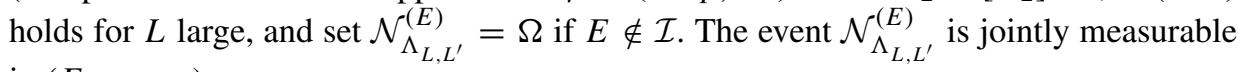
in $\left(E, \omega_{\Lambda_{L, L^{\prime}}}\right)$, so

$$
\mathcal{N}_{\Lambda_{L, L^{\prime}}}=\bigcap_{E \in \sigma\left(H_{\Lambda_{L^{\prime}}, \omega}\right)} \mathcal{N}_{\Lambda_{L, L^{\prime}}}^{(E)} \in \mathcal{F}_{\Lambda_{L}}
$$

and it follows from (6.59) and (6.34) that

$$
\mathbb{P}\left\{\mathcal{N}_{\Lambda_{L, L^{\prime}}}\right\}>1-C_{d, V_{\mathrm{per}}, \sup \mathcal{I}} L^{-4 b d} .
$$

Given a box $\Lambda_{L}\left(x_{0}\right)$, we define the multi-spectrum of the operator $H_{\omega}$, in the energy interval $\mathcal{I}$, by

$$
\Sigma_{H_{\omega}, L, x_{0}}^{(k)}:=\prod_{n=k}^{n_{1}} \sigma^{(\mathcal{I})}\left(H_{\omega, \Lambda_{L_{n}}\left(x_{0}\right)}\right) \quad \text { for } k=0,1, \ldots, n_{1} .
$$

A "multi-eigenvalue" $\boldsymbol{E}^{(k)}=\left\{E_{n}\right\}_{n=k}^{n_{1}} \in \boldsymbol{\Sigma}_{H_{\boldsymbol{\omega}}, L, x_{0}}^{(k)}$ will be called linked if

$$
\left|E_{n}-E_{n^{\prime}}\right| \leq 4 \mathrm{e}^{-\widehat{m} L_{\max \left\{n, n^{\prime}\right\}}} \quad \text { for all } n, n^{\prime} \in\left\{k, k+1, \ldots, n_{1}\right\} .
$$

The reduced multi-spectrum is then defined as

$$
\boldsymbol{\Sigma}_{H_{\omega}, L, x_{0}}^{(k, \text { red })}:=\left\{\boldsymbol{E}^{(k)} \in \boldsymbol{\Sigma}_{H_{\omega}, L, x_{0}}^{(k)} ; \boldsymbol{E}^{(k)} \text { is linked }\right\}, \quad k=0,1, \ldots, n_{1} .
$$


Lemma 6.11. Given a (sufficiently large) scale $L$ and $x_{0} \in \mathbb{R}^{d}$, consider the event

$$
\mathcal{N}_{L, x_{0}}:=\mathcal{N}_{\Lambda_{L_{0}, L_{1}}\left(x_{0}\right)} \cap\left\{\bigcap_{n=1}^{n_{1}-1}\left\{\mathcal{N}_{\Lambda_{L_{n}, L_{n+1}}\left(x_{0}\right)} \cap \mathcal{N}_{\Lambda_{2 L_{n}, L_{n+1}}\left(x_{0}\right)}\right\}\right\} .
$$

Then $\mathcal{N}_{L, x_{0}} \in \mathcal{F}_{\Lambda_{L}\left(x_{0}\right)}$ and

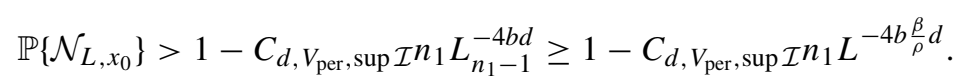

Moreover, for all $\omega \in \mathcal{N}_{L, x_{0}}$ we have

$$
\# \sigma^{(\mathcal{I}, \text { red })}\left(H_{\omega, \Lambda_{L}\left(x_{0}\right)}\right) \leq \# \Sigma_{H_{\omega}, L, x_{0}}^{(0, \text { red }} \leq C_{d, V_{\text {per }}, \mathcal{I}, p, \rho, n_{1}} L^{\left(n_{1}+1\right) \beta d} .
$$

Proof. We have $\mathcal{N}_{L, x_{0}} \in \mathcal{F}_{\Lambda_{L}\left(x_{0}\right)}$ by construction. Since $\beta=\rho^{n_{1}}$, the estimate (6.66) follows immediately from (6.61).

The first inequality in (6.67) is obvious, we only need to estimate $\# \Sigma_{H_{\omega}, L, x_{0}}^{(0, \text { red) }}$ for $\omega \in \mathcal{N}_{L, x_{0}}$. We will write $\Lambda_{L}=\Lambda_{L}\left(x_{0}\right), \Lambda_{L_{n}}=\Lambda_{L_{n}}\left(x_{0}\right)$.

It follows from (6.34) that

$$
\# \Sigma_{H_{\omega}, L, x_{0}}^{\left(n_{1}, \text { red }\right)}=\# \sigma^{(\mathcal{I})}\left(H_{\omega, \Lambda_{L_{1}}}\right) \leq C_{d, V_{\mathrm{per}}, \sup \mathcal{I}}\left(L_{n_{1}}\right)^{d}=C_{d, V_{\mathrm{per}}, \sup \mathcal{I}} L^{\beta d} .
$$

Let $k \in\left\{1, \ldots, n_{1}\right\}$. We set $\widetilde{L}_{k-1}=L_{k-1}$ and $\widetilde{L}_{n}=\widehat{L}_{n}=2 L_{n}$ for $n=k, k+1, \ldots, n_{1}-$ 1 , and let $\Lambda_{\tilde{L}_{n}, L_{n+1}}=\Lambda_{L_{n}, L_{n+1}}\left(x_{0}\right)$. We take $\boldsymbol{E}^{(k)}=\left\{E_{n}\right\}_{n=k}^{n_{1}} \in \boldsymbol{\Sigma}_{H_{\omega}, L, x_{0}}^{(k, \text { red }}$. Since $\boldsymbol{\omega} \in$

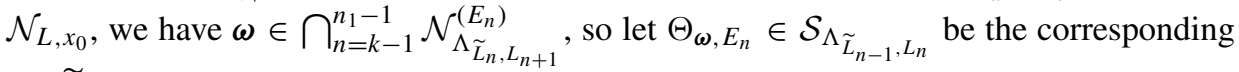
$\left(\omega, \widetilde{L}_{n-1}, L_{n}, E_{n}\right)$-singular set for $n=k, k+1, \ldots, n_{1}$, and set

$$
\Theta_{\omega, \boldsymbol{E}^{(k)}}=\Lambda_{2 L_{n_{1}}} \cup \bigcup_{n=k}^{n_{1}} \Theta_{\omega, E_{n}} .
$$

We have

$$
\left|\Theta_{\omega, \boldsymbol{E}^{(k)}}\right| \leq\left(2 L_{n_{1}}\right)^{d}+K_{2} \sum_{n=k}^{n_{1}}\left(3 \widetilde{L}_{n-1}^{\beta}\right)^{d} \leq 6^{d}\left(n_{1}-k+2\right) K_{2} L_{k-1}^{\beta d} .
$$

Given $k=1, \ldots, n_{1}$ and $\boldsymbol{E}^{(k)} \in \boldsymbol{\Sigma}_{H_{\omega}, L, x_{0}}^{(k, \text { red })}$, we set

$$
\Sigma_{H_{\omega}, L, x_{0}}^{(k-1)}\left(\boldsymbol{E}^{(k)}\right)=\left\{E \in \sigma^{(\mathcal{I})}\left(H_{\omega, \Lambda_{L_{k-1}}}\right) ;\left(E, \boldsymbol{E}^{(k)}\right) \in \boldsymbol{\Sigma}_{H_{\omega}, L, x_{0}}^{(k-1, \text { red }}\right\},
$$

and note that

$$
\# \boldsymbol{\Sigma}_{H_{\omega}, L, x_{0}}^{(k-1, \text { red })} \leq\left(\max _{\boldsymbol{E}^{(k)} \in \boldsymbol{\Sigma}_{H_{\omega}, L, x_{0}}^{(k, \text { red }}} \# \Sigma_{H_{\omega}, L, x_{0}}^{(k-1)}\left(\boldsymbol{E}^{(k)}\right)\right)\left(\# \boldsymbol{\Sigma}_{H_{\omega}, L, x_{0}}^{(k, \text { red })}\right) .
$$

We now fix $\boldsymbol{E}^{(k)} \in \Sigma_{H_{\omega}, L, x_{0}}^{(k, \text { red }}$. Given $E \in \Sigma_{H_{\omega}, L, x_{0}}^{(k-1)}\left(\boldsymbol{E}^{(k)}\right)$, let $\psi_{E}$ be a normalized eigenfunction of $H_{\boldsymbol{\omega}, \Lambda_{L_{k-1}}}$ corresponding to the eigenvalue $E$. If $x \in \Lambda_{L_{k-1}} \backslash \Theta_{\boldsymbol{\omega}, \boldsymbol{E}^{(k)}}$, there exist $n \in\left\{k, k+1, \ldots, n_{1}\right\}, j \in\left\{1, \ldots, n_{1}\right\}$, and an $\left(\omega, E_{n}, m, \varsigma\right)$-good box 
$\Lambda_{\ell_{n, j}} \subset \Lambda_{L_{k-1}}$, where $\ell_{n, j}=\left(\widetilde{L}_{n-1}\right)_{j} \geq L^{\rho^{n+j-1}}$, such that $\Lambda_{\ell_{n, j} / 5}(x) \cap \Lambda_{L_{k-1}} \subset \Lambda_{\ell_{n, j}}$. (This is ensured by our choice of the $\widetilde{L}_{n}$.) Since $\left|E-E_{n}\right| \leq 4 \mathrm{e}^{-\widehat{m} L_{n}}$, it follows from Lemma 3.7 that the box $\Lambda_{\ell_{n, j}}$ is $(\omega, E, \widehat{m} / 2, \varsigma)$-jgood, and hence we get, proceeding as in (3.22),

$$
\left\|\chi_{x} \psi_{E}\right\| \leq \mathrm{e}^{-\frac{\widehat{m}}{25} \ell_{n, j}} \leq \mathrm{e}^{-\frac{\widehat{m}}{25} L^{2 \rho^{2 n}-1}}
$$

so we conclude that

$$
\left\|\chi_{\Theta_{\boldsymbol{\omega}, \boldsymbol{E}}^{(k)}} \psi_{E}\right\|^{2} \geq 1-\mathrm{e}^{-\frac{2 \widehat{m}}{25} L^{\rho^{2 n} 1^{-1}}} L^{\rho^{k-1} d} \geq 1 / 2 .
$$

Thus,

$$
\begin{aligned}
\# \Sigma_{H_{\boldsymbol{\omega}}, L, x_{0}}^{(k-1)}\left(\boldsymbol{E}^{(k)}\right) & \leq 2 \operatorname{tr}\left\{\chi_{\mathcal{I}}\left(H_{\boldsymbol{\omega}, \Lambda_{L_{k-1}}}\right) \chi_{\Theta_{\omega, \boldsymbol{E}}^{(k)}}\right\} \leq 2 C_{d, V_{\mathrm{per}}, \mathcal{I}}\left|\Theta_{\omega, \boldsymbol{E}^{(k)}}\right| \\
& \leq C_{d, V_{\mathrm{per}}, \mathcal{I}, p, \rho, n_{1}} L_{k-1}^{\beta d},
\end{aligned}
$$

where we used [GK5, Lemma A.4] (as in (4.76)) and (6.70).

In view of (6.68) and (6.72), and recalling $\rho<1$, we get

$$
\# \boldsymbol{\Sigma}_{H_{\omega}, L, x_{0}}^{(0, \text { red })} \leq\left(C_{d, V_{\mathrm{per}}, \mathcal{I}, p, \rho, n_{1}}^{\prime} L^{\beta d}\right)^{n_{1}+1} \leq C_{d, V_{\mathrm{per}}, \mathcal{I}, p, \rho, n_{1}}^{\prime \prime} L^{\left(n_{1}+1\right) \beta d}
$$

We are now ready to prove Proposition 6.9.

Proof of Proposition 6.9. Setting

$$
\mathcal{X}_{L, x_{0}}:=\widetilde{\mathcal{Q}}_{L, x_{0}} \cap \mathcal{N}_{L, x_{0}},
$$

Proposition 6.9 is an immediate consequence of Lemmas 6.10 and 6.11.

\subsection{Annuli of good boxes}

We are now ready to prove Theorem 6.1.

Proposition 6.12. Given a sufficiently large scale $L$, for each $x_{0} \in \mathbb{R}^{d}$ there exists an event $\mathcal{U}_{L, x_{0}}$ as in (6.4) such that for all $\omega \in \mathcal{U}_{L, x_{0}}$, if $E \in \mathcal{I}_{L}$ satisfies (6.5), then every box $\Lambda_{L / 100}$ in the standard $L / 100$-covering of the annulus $\Lambda_{L_{+}, L_{-}}\left(x_{0}\right)$ is $(\omega, E, 70 \widehat{m}, \varsigma)$ jgood.

Proof. Given $E \in \mathcal{I}$, we let $\mathcal{M}_{L, x_{0}}^{(E)}$ be the event that all the boxes in the standard $L / 100$-covering of the annulus $\Lambda_{L_{+}, L_{-}}=\Lambda_{L_{+}, L_{-}}\left(x_{0}\right)$ are $(\omega, E, m, \varsigma)$-good, and set $\mathcal{M}_{L, x_{0}}^{(E)}=\Omega$ if $E \notin \mathcal{I}$. The event is jointly measurable in $\left(E, \omega_{\Lambda_{L_{+}, L_{-}}}\right)$, and, using (3.69),

$$
\mathbb{P}\left\{\mathcal{M}_{L, x_{0}}^{(E)}\right\}>1-(2002)^{d}(100)^{p d} L^{-p d} \quad \text { for } E \in \mathcal{I}
$$

Setting

$$
\mathcal{M}_{L, x_{0}}=\bigcap_{E \in \sigma^{(\mathcal{I}, \text { red })}\left(H_{\omega, \Lambda_{L-}\left(x_{0}\right)}\right)} \mathcal{M}_{L, x_{0}}^{(E)} \in \mathcal{F}_{\Lambda_{L_{+}}\left(x_{0}\right)},
$$


it follows from (6.55) and (6.78) that

$$
\begin{aligned}
\mathbb{P}\left\{\mathcal{M}_{L, x_{0}}\right\} & >1-C_{d, V_{\mathrm{per}}, \mathcal{I}, p, \rho, n_{1}}(2002)^{d}(100)^{p d} L^{-p d} L^{\left(n_{1}+1\right) \beta d} \\
& \geq 1-C_{d, V_{\mathrm{per}}, \mathcal{I}, p, \rho, n_{1}}^{\prime} L^{-\left(p-\left(n_{1}+1\right) \beta\right) d} .
\end{aligned}
$$

We now require that $K$, fixed in Proposition 6.3 subject only to the condition $K \geq$ $K_{d, p, b}$, is sufficiently large to ensure that, given a scale $L$, if $E \in \mathcal{I}_{L}$ satisfies (6.5), then $E$ satisfies (6.53) at scale $L_{-}$:

$$
\mathrm{e}^{-M L^{\beta / 2}} \geq \mathrm{e}^{-\widehat{m} \sqrt{L_{-}^{\beta} / K}}, \quad \text { i.e., } \quad K \geq 900\left(\frac{499}{500}\right)^{\beta} .
$$

We introduce the event

$$
\mathcal{U}_{L, x_{0}}=\mathcal{X}_{L_{-}, x_{0}} \cap \mathcal{M}_{L, x_{0}} \in \mathcal{F}_{\Lambda_{L_{+}}\left(x_{0}\right)},
$$

where $\mathcal{X}_{L_{-}, x_{0}}$ is the event given in Proposition 6.9 with $b=1+\frac{1}{\beta}\left(p-\left(n_{1}+1\right) \beta\right)$. It follows from (6.52), (6.80) and (6.2) that

$$
\mathbb{P}\left\{\mathcal{U}_{L, x_{0}}\right\}>1-L_{-}^{-b \beta d}-C_{d, V_{\mathrm{per}}, \mathcal{I}, p, \rho, n_{1}}^{\prime} L^{-\left(p-\left(n_{1}+1\right) \beta\right) d} \geq 1-L^{-\tilde{p} d}
$$

Fix $\omega \in \mathcal{U}_{L, x_{0}}$, and let $E \in \mathcal{I}_{L}$ satisfy (6.5), so it follows that (6.53) holds at scale $L_{-}$. Proposition 6.9 then gives (6.54) at scale $L_{-}$:

$$
\operatorname{dist}\left(E, \sigma^{(\mathcal{I}, \text { red })}\left(H_{\omega, \Lambda_{L_{-}}\left(x_{0}\right)}\right)\right) \leq e^{-\widehat{m} L_{-}}=\mathrm{e}^{-\frac{499 \widehat{m}}{5} \frac{L}{100}} .
$$

Thus, given a box $\Lambda_{L / 100}$ in the standard $L / 100$-covering of the annulus $\Lambda_{L_{+}, L_{-}}\left(x_{0}\right)$, it follows from (6.79) that the box $\Lambda_{L / 100}$ is $(\omega, E, m, \varsigma)$-good for all energies $E \in$ $\sigma^{(\mathcal{I} \text {,red) }}\left(H_{\omega, \Lambda_{L_{-}}\left(x_{0}\right)}\right)$. We conclude from (6.84) and Lemma 3.7 that the box $\Lambda_{L / 100}$ is $(\omega, E, 70 \widehat{m}, \varsigma)$-jgood.

Proof of Theorem 6.1. The theorem follows from Proposition 6.12, with $\mathcal{U}_{L, x_{0}}$ the event given in Proposition 6.12.

We fix $\omega \in \mathcal{U}_{L, x_{0}}$ and $E \in \mathcal{I}_{L}$. Recall $\vartheta=\beta / 2$.

If (6.5) holds, Proposition 6.12 guarantees that every box $\Lambda_{L / 100}$ in the standard $L / 100$-covering of the annulus $\Lambda_{L_{+}, L_{-}}\left(x_{0}\right)$ is $(\omega, E, 70 \widehat{m}, \varsigma)$-jgood, so it follows from Lemma 5.4 that

$$
W_{\boldsymbol{\omega}, x, L}(E) \leq \mathrm{e}^{-\frac{70 \widehat{m}}{2000} L} \leq \mathrm{e}^{-\frac{\widehat{m}}{30} L}=\mathrm{e}^{-M L},
$$

proving (6.6) (5.6),

To prove (6.7), note that either $E$ satisfies (6.5), so we have (6.6), and hence, recalling

or we have

$$
W_{\boldsymbol{\omega}, x_{0}}(E) W_{\boldsymbol{\omega}, x_{0}, L}(E) \leq 2^{v / 2} \mathrm{e}^{-M L},
$$

$$
\text { or we have } W_{\boldsymbol{\omega}, x_{0}}(E) \leq \mathrm{e}^{-M L^{\vartheta}},
$$

so using (5.12) we get

$$
W_{\boldsymbol{\omega}, x_{0}}(E) W_{\boldsymbol{\omega}, x_{0}, L}(E) \leq 2^{v / 2} L^{v} \mathrm{e}^{-M L^{\vartheta}} \leq \mathrm{e}^{-\frac{1}{2} M L^{\vartheta}} .
$$

The desired (6.7) follows. 
Remark 6.13. If $p>1$, the proof of Theorem 6.1 is much simpler; it does not require the second energy reduction of Proposition 6.9. The event $\mathcal{M}_{L, x_{0}}$ in (6.79) is replaced by

$$
\widetilde{\mathcal{M}}_{L, x_{0}}=\bigcap_{E \in \sigma(\mathcal{I})} \bigcap_{\left(H_{\Lambda_{L}\left(x_{0}\right), \omega}\right)} \mathcal{M}_{L, x_{0}}^{(E)} \in \mathcal{F}_{\Lambda_{L_{+}}\left(x_{0}\right)},
$$

so we have

$$
\mathbb{P}\left\{\widetilde{\mathcal{M}}_{L, x_{0}}\right\}>1-(2002)^{d}(100)^{p d} L^{-p d} C_{d, V_{\mathrm{per}}, \mathcal{I}} L^{d} \geq 1-C_{d, V_{\mathrm{per}}, \mathcal{I}}^{\prime} L^{-(p-1) d} .
$$

The event $\mathcal{U}_{L, x_{0}}$ in (6.82) is replaced by

$$
\tilde{\mathcal{U}}_{L, x_{0}}=\mathcal{Q}_{L, x_{0}} \cap \widetilde{\mathcal{M}}_{L, x_{0}} \in \mathcal{F}_{\Lambda_{L_{+}}\left(x_{0}\right)},
$$

where $\mathcal{Q}_{L, x_{0}}$ is the event given in Proposition 6.3. It follows from (6.8) and (6.90) that

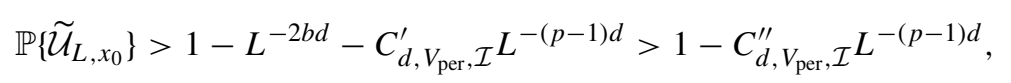

choosing $b=1+(p-1) / 2$. The proof of Theorem 6.1 then proceeds as before, with $\vartheta=1$ in (6.5) and (6.7).

Remark 6.14. If $p>3$, we can prove a modified version of Theorem 6.1 that does not require either Proposition 6.3 or Proposition 6.9; it suffices to use Lemma 6.6. The conditions $E \in \mathcal{I}_{L}$ and (6.5) are replaced by

$$
E \in \mathcal{I} \quad \text { and } \quad W_{\omega, x_{0}}(E)>\mathrm{e}^{-\frac{m}{30} \sqrt{L}} .
$$

We replace the event $\mathcal{M}_{L, x_{0}}^{(E)}$ by $\widehat{\mathcal{M}}_{L, x_{0}}^{(E)}$, the event that all the boxes in the standard $\sqrt{L}$ covering of the annulus $\Lambda_{L_{+}, L_{-}}\left(x_{0}\right)$ are $(\omega, E, m, \varsigma)$-good, and set $\widehat{\mathcal{M}}_{L, x_{0}}^{(E)}=\Omega$ if $E \notin \mathcal{I}$. We have

$$
\mathbb{P}\left\{\widehat{\mathcal{M}}_{L, x_{0}}^{(E)}\right\}>1-20^{d} L^{\frac{1}{2} d} L^{-\frac{p}{2} d}=1-20^{d} L^{-\frac{p-1}{2} d} \quad \text { for } E \in \mathcal{I} .
$$

We set

$$
\widehat{\mathcal{M}}_{L, x_{0}}=\bigcap_{E \in \sigma(\mathcal{I})} \bigcap_{\left(H_{\Lambda_{L}\left(x_{0}\right), \omega}\right)} \widehat{\mathcal{M}}_{L, x_{0}}^{(E)} \in \mathcal{F}_{\Lambda_{L_{+}}\left(x_{0}\right)},
$$

so we have

$$
\mathbb{P}\left\{\widehat{\mathcal{M}}_{L, x_{0}}\right\}>1-20^{d} L^{-\frac{p-1}{2} d} C_{d, V_{\mathrm{per}}, \mathcal{I}} L^{d} \geq 1-C_{d, V_{\mathrm{per}}, \mathcal{I}^{\prime \prime}} L^{-\frac{p-3}{2} d} .
$$

The event $\mathcal{U}_{L, x_{0}}$ in (6.82) is replaced by

$$
\widehat{\mathcal{U}}_{L, x_{0}}=\mathcal{T}_{L, x_{0}} \cap \widehat{\mathcal{M}}_{L, x_{0}} \in \mathcal{F}_{\Lambda_{L_{+}}\left(x_{0}\right)},
$$

where $\mathcal{T}_{L, x_{0}}$ is the event in Lemma 6.6. It follows from (6.23) and (6.96) that

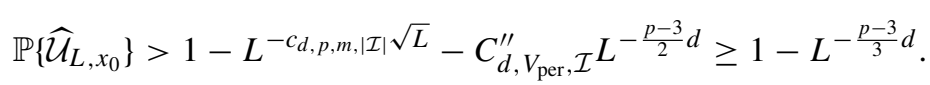


The proof of Proposition 6.12 then proceeds as before, except that we use Lemma 6.6 and boxes of side $\sqrt{L}$ instead of $L / 100$. We conclude, using Lemma 3.7, that if $E$ satisfies (6.93), then all the boxes in the standard $\sqrt{L}$-covering of the annulus $\Lambda_{L_{+}, L_{-}}\left(x_{0}\right)$ are $(\omega, E, m / 60, \varsigma)$-jgood. Applying Lemma 5.4, modified for boxes of side $\sqrt{L}$ instead of $L / 100$, we obtain (cf. (6.6))

$$
W_{\boldsymbol{\omega}, x_{0}, L}(E) \leq \mathrm{e}^{-\frac{m}{1000} \sqrt{L}} .
$$

It follows that we have (cf. (6.7))

$$
W_{\boldsymbol{\omega}, x_{0}}(E) W_{\boldsymbol{\omega}, x_{0}, L}(E) \leq \mathrm{e}^{-\frac{m}{1000} \sqrt{L}} \quad \text { for all } E \in \mathcal{I} .
$$

This simpler result implies pure point spectrum with subexponential decay of eigenfunctions, as well as dynamical localization.

\section{Localization}

In this section we derive all the usual forms of localization from Theorem 6.1. We will assume only the conclusions of this theorem. More precisely, we will assume only the existence of the events $\mathcal{U}_{L, 0}$ satisfying the conclusions of Theorem 6.1 for some fixed $\tilde{p}, \vartheta, M$. In particular, we do not assume the conclusions of the multiscale analysis, which were the hypotheses for Theorem 6.1. We fix $v>d / 2$, which will be generally omitted from the notation.

\subsection{Anderson localization and finite multiplicity of eigenvalues}

A simple Borel-Cantelli Lemma argument based on Theorem 6.1 yields Anderson localization and finite multiplicity of eigenvalues. We only need the events of Theorem 6.1 at $x_{0}=0$.

Theorem 7.1. Let $H_{\omega}$ be a generalized Anderson Hamiltonian on $\mathrm{L}^{2}\left(\mathbb{R}^{d}\right)$. Let $\mathcal{I} \subset \mathbb{R}$ be a bounded open interval, for which there is a scale $\mathcal{L}_{1}$ such that for all $L \geq \mathcal{L}_{1}$ there exists an event $\mathcal{U}_{L, 0}$ as in Theorem 6.1. Then the following holds with probability one:

(i) $H_{\omega}$ has pure point spectrum in the interval $\mathcal{I}$.

(ii) If $\psi$ is an eigenfunction of $H_{\omega}$ with eigenvalue $E \in \mathcal{I}$, then $\psi$ is exponentially localized with rate of decay $M$, more precisely,

$$
\left\|\chi_{x} \psi\right\| \leq C_{\omega, E}\left\|T^{-1} \psi\right\| e^{-M\|x\|} \quad \text { for all } x \in \mathbb{R}^{d} .
$$

(iii) For all $E \in \mathcal{I}$ we have

$$
\left\|\chi_{x} P_{\omega}(E)\right\|_{2} \leq C_{\omega, E}^{\prime} \mathrm{e}^{-M\|x\|} \quad \text { for all } x \in \mathbb{R}^{d} .
$$

(iv) The eigenvalues of $H_{\omega}$ in $\mathcal{I}$ have finite multiplicity:

$$
\operatorname{tr} P_{\omega}(E)<\infty \quad \text { for all } E \in \mathcal{I} \text {. }
$$


Proof. It suffices to prove the theorem in every closed interval $I \subset \mathcal{I}$. We fix $I$, and pick a scale $L_{0} \geq \mathcal{L}_{1}$ such that $I \subset \mathcal{I}_{L_{0}}$ (see (6.3)). We introduce scales $L_{k+1}=2 L_{k}$ for $k=1, \ldots$, and set $\mathcal{U}_{k}=\mathcal{U}_{L_{k}, 0}$. It follows from the Borel-Cantelli Lemma, using (6.4), that

$$
\mathbb{P}\left\{\mathcal{U}_{\infty}\right\}=1, \quad \text { where } \quad \mathcal{U}_{\infty}=\liminf _{k \rightarrow \infty} \mathcal{U}_{k}
$$

Fix $\omega \in \mathcal{U}_{\infty}$; there exists $k_{\omega} \in \mathbb{N}$ such that $\omega \in \mathcal{U}_{k}$ for all $k \geq k_{\boldsymbol{\omega}}$. If $E \in I$ is a generalized eigenvalue of $H_{\omega}$, i.e., $\Theta_{\omega}(E) \neq \emptyset$, and hence $W_{\omega, 0}(E)>0$, we set

$k_{\omega, E}=\min \left\{k \in \mathbb{N} ; k \geq k_{\omega}\right.$ and (6.5) holds for $E$ and $L_{k}\left(\right.$ with $\left.\left.x_{0}=0\right)\right\}<\infty$.

Given $\psi \in \Theta_{\omega}(E)$, it follows from (6.6) that

$$
\left\|\chi_{0, L_{k}} \psi\right\| \leq\left\|T^{-1} \psi\right\| \mathrm{e}^{-M L_{k}} \quad \text { for all } k \geq k_{\omega, E}
$$

If $x \in \mathbb{R}^{d}$ with $\|x\| \geq L_{k_{\omega, E}}$, we can always find $k \geq k_{\omega, E}$ such that $x \in \bar{\Lambda}_{L_{k+1}, L_{k}}(0)$, so

$$
\left\|\chi_{x} \psi\right\| \leq\left\|\chi_{0, L_{k}} \psi\right\| \leq\left\|T^{-1} \psi\right\| \mathrm{e}^{-M L_{k}} \leq\left\|T^{-1} \psi\right\| \mathrm{e}^{-M\|x\|} .
$$

It follows that that $\psi \in \mathcal{H}=\mathrm{L}^{2}\left(\mathbb{R}^{d}\right)$ and satisfies (7.1). It now follows from (5.25) that (5.26) holds with $B=I$. We conclude that $H_{\omega}$ has pure point spectrum in $I$, and if $\psi$ is an eigenfunction of $H_{\omega}$ with eigenvalue $E \in I$ it has the exponential decay given in (7.1).

The estimate (7.2) is an immediate consequence of (7.1), and implies (7.3).

\subsection{Eigenfunctions correlations and dynamical localization}

Another Borel-Cantelli Lemma argument based on Theorem 6.1 yields eigenfunctions correlations. In particular, we obtain pure point spectrum, finite multiplicity of eigenvalues, SUDEC (summable uniform decay of eigenfunction correlations; see [GK6]) and SULE (semi-uniformly localized eigenfunctions; see [DeRJLS, GK1, GK6]), and dynamical localization. We will need the events of Theorem 6.1 for all $x \in \mathbb{Z}^{d}$. We do not assume or use Theorem 7.1.

Theorem 7.2. Let $H_{\omega}$ be a generalized Anderson Hamiltonian on $\mathrm{L}^{2}\left(\mathbb{R}^{d}\right)$. Let $\mathcal{I} \subset \mathbb{R}$ be a bounded open interval for which there is a scale $\mathcal{L}_{2}$ such that for all $L \geq \mathcal{L}_{2}$ and $x \in \mathbb{Z}^{d}$ there exists an event $\mathcal{U}_{L, x}$ as in Theorem 6.1. Let $\varepsilon>0$ and fix an open interval $I \subset \bar{I} \subset \mathcal{I}$. The following holds with probability one:

(i) For all $E \in I$ we have

$$
W_{\boldsymbol{\omega}, x}(E) W_{\boldsymbol{\omega}, y}(E) \leq C_{\boldsymbol{\omega}, I, \varepsilon} \mathrm{e}^{\|x\|^{(1+\varepsilon) \vartheta / \widetilde{p}}} \mathrm{e}^{-\frac{1}{3} M\|x-y\|^{\vartheta}} \quad \text { for all } x, y \in \mathbb{R}^{d} .
$$

(ii) $H_{\omega}$ has pure point spectrum in the interval I. Moreover, the eigenvalues of $H_{\omega}$ in $I$ have finite multiplicity. 
(iii) (SUDEC) For all $E \in I$ and $\phi, \psi \in \operatorname{Ran} P_{\omega}(E)$ we have

$$
\begin{aligned}
& \left\|\chi_{x} \phi\right\|\left\|\chi_{y} \psi\right\| \\
& \leq C_{\omega, I, \varepsilon}^{\prime}\left\|T^{-1} \phi\right\|\left\|T^{-1} \psi\right\| \mathrm{e}^{\|x\| \|^{(1+\varepsilon) \vartheta / \widetilde{p}}} \mathrm{e}^{-\frac{1}{4} M\|x-y\|^{\vartheta}} \quad \text { for all } x, y \in \mathbb{R}^{d} .
\end{aligned}
$$

In addition, for all $E \in I$ we have

$$
\begin{aligned}
& \left\|\chi_{x} P_{\omega}(E)\right\|_{2}\left\|\chi_{y} P_{\omega}(E)\right\|_{2} \\
& \quad \leq C_{\omega, I, \varepsilon}^{\prime} \mu_{\omega}(E) \mathrm{e}^{\|x\|^{(1+\varepsilon) \vartheta / \widetilde{p}}} \mathrm{e}^{-\frac{1}{4} M\|x-y\|^{\vartheta}} \quad \text { for all } x, y \in \mathbb{R}^{d} .
\end{aligned}
$$

(iv) (SULE) For all $E \in I$ there exists a center of localization $y_{\omega, E} \in \mathbb{R}^{d}$ for all eigenfunctions with eigenvalue E, i.e., for all $\phi \in \operatorname{Ran} P_{\omega}(E)$ we have

$$
\left\|\chi_{x} \phi\right\| \leq C_{\omega, I, \varepsilon}^{\prime \prime}\left\|T^{-1} \phi\right\| \mathrm{e}^{\left\|y_{\omega, E}\right\|^{(1+\varepsilon) \vartheta / \widetilde{p}}} \mathrm{e}^{-\frac{1}{4} M\left\|x-y_{\omega, E}\right\|^{\vartheta}} \quad \text { for all } x \in \mathbb{R}^{d} .
$$

In addition, for all $E \in I$ we have

$$
\begin{aligned}
& \left\|\chi_{x} P_{\omega}(E)\right\|_{2} \\
& \quad \leq C_{\omega, I, \varepsilon}^{\prime \prime} \sqrt{\mu_{\omega}(E)} \mathrm{e}^{\left\|y_{\omega, E}\right\|^{(1+\varepsilon) \vartheta / \widetilde{p}}} \mathrm{e}^{-\frac{1}{4} M\left\|x-y_{\omega, E}\right\|^{\vartheta}} \quad \text { for all } x \in \mathbb{R}^{d} .
\end{aligned}
$$

(v) We have

$$
N_{\omega, I}(L):=\sum_{E \in I ;\left\|y_{\omega, E}\right\| \leq L} \operatorname{tr} P_{\omega}(E) \leq C_{\omega, I, \varepsilon} L^{(1+\varepsilon) d / \widetilde{p}} \quad \text { for all } L \geq 1 .
$$

Proof. Fix $\varepsilon>0$. Given $k \in \mathbb{N}$, we set $L_{k}=2^{k}$, and consider the event

$$
\mathcal{J}_{k}:=\bigcap_{x \in \mathbb{Z}^{d} ;\|x\|^{1+\varepsilon} \leq \tau L_{k}^{\tilde{p}}} \mathcal{U}_{L_{k}, x}
$$

where $\mathcal{U}_{L, x}, M, \tilde{p}, \beta$ are as in Theorem 6.1 , and $\tau>0$ is a constant to be chosen later. It follows from (6.4) that

$$
\mathbb{P}\left\{\mathcal{J}_{k}\right\} \geq 1-C_{d, M, \varepsilon, \tau} L_{k}^{-\frac{\varepsilon}{1+\varepsilon} \widetilde{p} d} .
$$

Applying the Borel-Cantelli Lemma we conclude that

$$
\mathbb{P}\left\{\mathcal{J}_{\infty}\right\}=1, \quad \text { where } \quad \mathcal{J}_{\infty}=\liminf _{k \rightarrow \infty} \mathcal{J}_{k}
$$

Thus, for $\omega \in \mathcal{J}_{\infty}$ there exists $k_{1}(\boldsymbol{\omega}) \in \mathbb{N}$ such that $\omega \in \mathcal{U}_{L_{k}, x}$ for all $k \geq k_{1}(\boldsymbol{\omega})$ and $x \in \mathbb{Z}^{d}$ with $\|x\|^{1+\varepsilon} \leq \tau L_{k}^{\tilde{p}}$.

We now fix $\omega \in \mathcal{J}_{\infty}$ and an open interval $I \subset \bar{I} \subset \mathcal{I}$. We set

$$
k_{1}(\omega, I)=\min \left\{k \in \mathbb{N} ; k \geq k_{1}(\omega), k \geq 2, I \subset \mathcal{I}_{L_{k}}\right\},
$$

where $\mathcal{I}_{L}$ is defined in (6.3). Given $x \in \mathbb{Z}^{d}$, we define $k_{2}(x) \in \mathbb{N}, k_{2}(x) \geq 2$, by

$$
\tau L_{k_{2}(x)-1}^{\widetilde{p}}<\|x\|^{1+\varepsilon} \leq \tau L_{k_{2}(x)}^{\widetilde{p}},
$$


when possible, and set $k_{2}(x)=1$ otherwise. We let $k_{3}(\omega, I, x)=\max \left\{k_{1}(\omega, I), k_{2}(x)\right\}$; note that $k_{3}(\omega, I, x) \geq 2$. It follows from (6.7), using (5.13), that for all $E \in I$ and $y \in \mathbb{R}^{d} \backslash \Lambda_{L_{k_{3}(\omega, I, x)}}(x)$ we have

$$
W_{\omega, x}(E) W_{\omega, y}(E) \leq 2^{\nu}\|x-y\|^{\nu} \mathrm{e}^{-\frac{1}{2} M\|x-y\|^{\vartheta}} .
$$

If $y \in \Lambda_{L_{k_{3}(\omega, I, x)}}(x)$, we have

$$
\begin{aligned}
& W_{\boldsymbol{\omega}, x}(E) W_{\boldsymbol{\omega}, y}(E)=W_{\boldsymbol{\omega}, x}(E) W_{\boldsymbol{\omega}, y}(E) \mathrm{e}^{\frac{1}{2} M\|x-y\|^{\vartheta}} \mathrm{e}^{-\frac{1}{2} M\|x-y\|^{\vartheta}} \\
& \leq 2^{\nu} \mathrm{e}^{\frac{1}{2} M\left(\frac{1}{2} L_{\left.k_{3}(\omega, I, x)\right)^{\vartheta}}\right.} \mathrm{e}^{-\frac{1}{2} M\|x-y\|^{\vartheta}} \leq 2^{\nu} \mathrm{e}^{\frac{1}{2} M L_{k_{3}(\omega, I, x)-1}^{\vartheta}} \mathrm{e}^{-\frac{1}{2} M\|x-y\|^{\vartheta}} \\
& \leq \begin{cases}2^{v} \mathrm{e}^{\|x\| \|^{(1+\varepsilon) \vartheta / \tilde{p}}} \mathrm{e}^{-\frac{1}{2} M\|x-y\|^{\vartheta}} & \text { if } k_{3}(\omega, I, x)=k_{2}(x), \\
2^{v} \mathrm{e}^{\frac{1}{2} M L_{k_{1}(\omega, I)-1}} \mathrm{e}^{-\frac{1}{2} M\|x-y\|^{\vartheta}} & \text { if } k_{3}(\omega, I, x)=k_{1}(\omega, I),\end{cases}
\end{aligned}
$$

where we used (5.6) and made an appropriate chice of the constant $\tau$. The estimate (7.8) follows from (7.19) and (7.20).

It follows from (7.8) that for all $E \in I$ and all $\phi, \psi \in \widetilde{\Theta}_{\omega}(E)$ we have, for all $x, y \in \mathbb{R}^{d}$,

$$
\begin{aligned}
\left\|\chi_{x} \phi\right\|\left\|\chi_{y} \psi\right\| & \leq C_{\omega, I, \varepsilon}\left\|T_{x}^{-1} \phi\right\|\left\|T_{y}^{-1} \psi\right\| \mathrm{e}^{\|x\|^{(1+\varepsilon) \vartheta / \widetilde{p}}} \mathrm{e}^{-\frac{1}{3} M\|x-y\|^{\vartheta}} \\
& \leq 2^{\nu} C_{\omega, I, \varepsilon}\langle x\rangle^{\nu}\langle y\rangle^{\nu}\left\|T^{-1} \phi\right\|\left\|T^{-1} \psi\right\| \mathrm{e}^{(1+\varepsilon) \vartheta / \widetilde{p}} \mathrm{e}^{-\frac{1}{3} M\|x-y\|^{\vartheta}} .
\end{aligned}
$$

Thus $\widetilde{\Theta}_{\omega}(E) \subset \mathcal{H}$ for all $E \in I$. It now follows from (5.25) that (5.26) holds with $B=I$, and hence $H_{\omega}$ has pure point spectrum in $I$. The estimate (7.9) follows from (7.21). The estimate (7.10) is an immediate consequence of (7.9), and implies $\operatorname{tr} P_{\omega}(E)<\infty$ for all $E \in I$.

Given $E \in I$ with $P_{\omega}(E) \neq 0$, we pick $\psi \in \operatorname{Ran} P_{\omega}(E), \psi \neq 0$, and pick $y_{\omega, E} \in \mathbb{Z}^{d}$ (not unique) such that

$$
\left\|\chi_{y_{\omega, E}} \psi\right\|=\max _{y \in \mathbb{Z}^{d}}\left\|\chi_{y} \psi\right\| .
$$

It follows that (see [GK6, Eq. (4.22)])

$$
W_{\omega, y_{\omega, E}}(E) \geq \frac{\left\|\chi_{y_{\omega, E}} \psi\right\|}{\left\|T_{y_{\omega, E}}^{-1} \psi\right\|} \geq C_{d}>0
$$

If $P_{\omega}(E)=0$ we take $y_{\omega, E} \in \mathbb{Z}^{d}=0$. Then for all $E \in I$ and all $\psi \in \operatorname{Ran} P_{\omega}(E)$, (7.11) and (7.12) follow from (7.8) (taking $y=y_{\omega, E}$ ) and (7.23).

To prove (7.13), note that it follows from (7.12) that for all $E \in I$ and $R \geq 1$ we have

$$
\begin{aligned}
\left\|\chi_{\mathbb{R}^{d} \backslash \Lambda_{2 R}\left(y_{\omega, E}\right)} P_{\omega}(E)\right\|_{2}^{2} & \leq \sum_{x \in \mathbb{Z}^{d} \backslash \Lambda_{2 R-1}\left(y_{\omega, E}\right)}\left\|\chi_{x} P_{\omega}(E)\right\|_{2}^{2} \\
& \leq C_{\omega, I, \varepsilon} \mu_{\omega}(I) \mathrm{e}^{2\left\|y_{\omega, E}\right\|^{(1+\varepsilon) \vartheta / \widetilde{p}}} \mathrm{e}^{-\frac{1}{2} M(R / 2)^{\vartheta}} \\
& =C_{\omega, I, \varepsilon}^{\prime} \mathrm{e}^{2\left\|y_{\omega, E}\right\|^{(1+\varepsilon) \vartheta / \widetilde{p}}} \mathrm{e}^{-\frac{1}{2} M(R / 2)^{\vartheta}} .
\end{aligned}
$$


There is a constant $D_{\omega, I, \varepsilon} \geq 1$ such that for all $L \geq 1$,

$$
R \geq D_{\omega, I, \varepsilon} L^{(1+\varepsilon) / \widetilde{p}} \Rightarrow C_{\omega, I, \varepsilon}^{\prime} \mathrm{e}^{2 L^{(1+\varepsilon) \vartheta / \widetilde{p}}} \mathrm{e}^{-\frac{1}{2} M(R / 2)^{\vartheta}} \leq 1 / 2 .
$$

Thus, given $L \geq 1$, letting $R_{L}:=D_{\omega, I, \varepsilon} L^{(1+\varepsilon) / \tilde{p}}$, we have

$$
\left\|\chi_{\Lambda_{2 R_{L}}\left(y_{\omega, E}\right)} P_{\omega}(E)\right\|_{2}^{2}>1 / 2 \quad \text { whenever } \quad\left\|y_{\omega, E}\right\| \leq L .
$$

It follows, using also (5.21), that

$$
\begin{aligned}
N_{\boldsymbol{\omega}, I}(L) & \leq 2 \sum_{\substack{E \in I \\
\left\|y_{\boldsymbol{\omega}, E}\right\| \leq L}}\left\|\chi_{\Lambda_{2 R_{L}}\left(y_{\omega, E}\right)} P_{\omega}(E)\right\|_{2}^{2} \leq 2 \sum_{\substack{E \in I \\
\left\|y_{\omega, E}\right\| \leq L}}\left\|\chi_{\Lambda_{2\left(L+R_{L}\right)}(0)} P_{\omega}(E)\right\|_{2}^{2} \\
& \leq 2\left\|\chi_{\Lambda_{2\left(L+R_{L}\right)}(0)} P_{\omega}(I)\right\|_{2}^{2} \leq C_{I}\left(L+R_{L}\right)^{d} \leq C_{\omega, I, \varepsilon} L^{(1+\varepsilon) d / \tilde{p}}
\end{aligned}
$$

We can now prove dynamical localization with probability one.

Corollary 7.3. Let $H_{\omega}$ be a generalized Anderson Hamiltonian satisfying the hypotheses of Theorem 7.2 in a bounded open interval $\mathcal{I}$. Let $\varepsilon>0$ and fix an open interval I $\subset$ $\bar{I} \subset \mathcal{I}$. The following holds with probability one:

(i) For all $E \in I$ we have

$$
\left\|\chi_{y} P_{\omega}(E) \chi_{x}\right\|_{1} \leq C_{\omega, I, \varepsilon}^{\prime} \mu_{\omega}(E) \mathrm{e}^{\|x\|^{(1+\varepsilon) \vartheta / \widetilde{p}}} \mathrm{e}^{-\frac{1}{4} M\|x-y\|^{\vartheta}} \quad \text { for all } x, y \in \mathbb{R}^{d} .
$$

(ii) We have

$$
\begin{aligned}
\sup _{f \in \mathcal{B}_{b, 1}} \| \chi_{y} f\left(H_{\omega}\right) & P_{\omega}(I) \chi_{x} \|_{1} \\
& \leq C_{\omega, I, \varepsilon}^{\prime \prime} \mathrm{e}^{\|x\|^{(1+\varepsilon) \vartheta / \widetilde{p}}} \mathrm{e}^{-\frac{1}{4} M\|x-y\|^{\vartheta}} \quad \text { for all } x, y \in \mathbb{R}^{d} .
\end{aligned}
$$

(iii) For all $b>0$ and $x_{0} \in \mathbb{R}^{d}$ we have

$$
\sup _{f \in \mathcal{B}_{b, 1}}\left\|\left\langle X-x_{0}\right\rangle^{b d} f\left(H_{\boldsymbol{\omega}}\right) P_{\boldsymbol{\omega}}(I) \chi_{x_{0}}\right\|_{1} \leq C_{\boldsymbol{\omega}, I, \varepsilon, b} \mathrm{e}^{\left\|x_{0}\right\|^{(1+\varepsilon) \vartheta / \widetilde{p}}},
$$

and, in particular,

$$
\sup _{t \in \mathbb{R}}\left\|\left\langle X-x_{0}\right\rangle^{b d} \mathrm{e}^{-i t H_{\omega}} P_{\omega}(I) \chi_{x_{0}}\right\|_{1} \leq C_{\omega, I, \varepsilon, b} \mathrm{e}^{\left\|x_{0}\right\|^{(1+\varepsilon) \vartheta / \widetilde{p}}} .
$$

(iv) For all $E \in I$ we have

$$
\left\|\chi_{y} P_{\omega}^{(E)} \chi_{x}\right\|_{1} \leq C_{\omega, I, \mathcal{I}, \varepsilon} \mathrm{e}^{\|x\|^{(1+\varepsilon) \vartheta / \widetilde{p}}} \mathrm{e}^{-\frac{1}{4} M\|x-y\|^{\vartheta}} \quad \text { for all } x, y \in \mathbb{R}^{d} .
$$


Proof. Since

$$
\left\|\chi_{x} P_{\omega}(E) \chi_{y}\right\|_{1} \leq\left\|\chi_{x} P_{\omega}(E)\right\|_{2}\left\|\chi_{y} P_{\omega}(E)\right\|_{2},
$$

(7.28) follows immediately from (7.10).

Given $f \in \mathcal{B}_{b, 1}$, it follows from (5.37) and (7.28) that

$$
\begin{aligned}
\left\|\chi_{y} f\left(H_{\omega}\right) P_{\omega}(I) \chi_{x}\right\|_{1} & \leq \int_{I}|f(E)|\left\|\chi_{y} P_{\omega, 0}(E) \chi_{x}\right\|_{1} \mathrm{~d} \mu_{\omega}(E) \\
& \leq C_{\omega, I, \varepsilon}^{\prime} \mu_{\omega}(I) \mathrm{e}^{\|x\|^{(1+\varepsilon) \vartheta / \widetilde{p}}} \mathrm{e}^{-\frac{1}{4} M\|x-y\|^{\vartheta}},
\end{aligned}
$$

which is (7.29).

Given $b>0$ and $x_{0} \in \mathbb{R}^{d}$, (7.30) and (7.31) follow from (7.29).

To prove (7.32), we proceed as in [GK6, Proof of Theorem 3]. We write $I=] \alpha_{1}, \alpha_{2}[$, let $\delta=\frac{1}{2} \operatorname{dist}(I, \mathbb{R} \backslash \mathcal{I})>0$, and consider the open interval $\left.I_{1}=\right] \alpha_{1}-\delta / 2, \alpha_{2}+\delta / 2[\subset$ $\overline{I_{1}} \subset \mathcal{I}$. We set $\left.\zeta=1 / 2(1+\beta / 2) \in\right] \beta / 2,1\left[\right.$ and $\left.\zeta^{\prime}=1 / 2(1+\zeta) \in\right] \zeta, 1[$. We pick an $\mathrm{L}^{1}$-Gevrey function $g$ of class $1 / \zeta^{\prime}$ on $]-1, \infty[$, such that $0 \leq g \leq 1, g \equiv 1$ on ]$\left.-\infty, \alpha_{1}-\delta / 2\right]$ and $g \equiv 0$ on $\left[\alpha_{2}+\delta / 2, \infty\right.$ [. (See [BGK, Definition 1.1]; such a function always exists.) For all $E \in I$ we have

$$
\begin{gathered}
P_{\omega}^{(E)}=g^{2}\left(H_{\omega}\right)+f_{E}\left(H_{\omega}\right), \quad \text { where } \\
f_{E}(t):=\chi_{]-\infty, E]}(t)-g^{2}(t)=f_{E}\left(H_{\omega}\right) P_{\omega}\left(I_{1}\right) \in \mathcal{B}_{b, 1} .
\end{gathered}
$$

Since we proved (7.29), we have

$$
\left\|\chi_{y} f_{E}\left(H_{\omega}\right) \chi_{x}\right\|_{1} \leq C_{\omega, I, \varepsilon}^{\prime \prime} \mathrm{e}^{\|x\|^{(1+\varepsilon) \vartheta / \widetilde{p}}} \mathrm{e}^{-\frac{1}{4} M\|x-y\|^{\vartheta}} \quad \text { for all } x, y \in \mathbb{R}^{d} .
$$

The function $g$ was chosen so that we can use [BGK, Theorem 1.4], obtaining

$$
\left\|\chi_{x} g\left(H_{\omega}\right) \chi_{y}\right\| \leq C_{g} \mathrm{e}^{-C_{g}^{\prime}\|x-y\|^{\zeta}} \quad \text { for all } x, y \in \mathbb{R}^{d} .
$$

We also have, using (5.21),

$$
\begin{aligned}
\left\|\chi_{x} g\left(H_{\omega}\right) \chi_{y}\right\|_{1} & \leq\left\|\chi_{x} \sqrt{g}\left(H_{\omega}\right)\right\|_{2}\left\|\chi_{y} \sqrt{g}\left(H_{\omega}\right)\right\|_{2} \leq\left\|\chi_{x} P_{\omega}^{\left(\alpha_{2}+\delta / 2\right)}\right\|_{2}\left\|\chi_{y} P_{\omega}^{\left(\alpha_{2}+\delta / 2\right)}\right\|_{2} \\
& \leq C_{d,\left\|V_{\text {per }}^{-}\right\|, \alpha_{2}+\delta / 2}^{2}
\end{aligned}
$$

It follows that

$$
\begin{aligned}
\left\|\chi_{x} g\left(H_{\omega}\right) \chi_{y}\right\|_{2}^{2} & \leq\left\|\chi_{x} g\left(H_{\omega}\right) \chi_{y}\right\|\left\|\chi_{x} g\left(H_{\omega}\right) \chi_{y}\right\|_{1} \\
& \leq C_{d,\left\|V_{\text {per }}^{-}\right\|, \alpha_{2}+\delta / 2, g} \mathrm{e}^{-C_{g}^{\prime}\|x-y\|^{\zeta}} .
\end{aligned}
$$

Thus, given $x, y \in \mathbb{R}^{d}$ we get

$$
\begin{aligned}
\left\|\chi_{x} g^{2}\left(H_{\omega}\right) \chi_{y}\right\|_{1} & \leq \sum_{z \in \mathbb{Z}^{d}}\left\|\chi_{x} g\left(H_{\omega}\right) \chi_{z}\right\|_{2}\left\|\chi_{z} g\left(H_{\omega}\right) \chi_{y}\right\|_{2} \\
& \leq C_{1} \sum_{z \in \mathbb{Z}^{d}} \mathrm{e}^{-\frac{1}{2} C_{g}^{\prime}\|x-z\|^{\zeta}} \mathrm{e}^{-\frac{1}{2} C_{g}^{\prime}\|z-y\|^{\zeta}} \leq C_{2} \mathrm{e}^{-C_{3}\|x-y\|^{\zeta}},
\end{aligned}
$$

where $C_{1}=C_{d,\left\|V_{\text {per }}^{-}\right\|, \alpha_{2}+\delta / 2, g}$ and $C_{2}, C_{3}$ depend only on $d,\left\|V_{\text {per }}^{-}\right\|, I, \mathcal{I}, \zeta$.

Since $\zeta>\beta / 2$, the estimate (7.32) now follows from (7.35)-(7.40). 


\subsection{Localization in expectation}

We will now derive eigenfunctions correlations estimates in expectation from Theorem 6.1 , and use them to get dynamical localization in expectation, as well as pure point spectrum, finite multiplicity of eigenvalues, etc., as in [GK6]. We do not assume or use the results of Subections 7.1 and 7.2.

We recall that we pick $v>d / 2$, and that $\boldsymbol{W}_{\boldsymbol{\omega}, x}(E)$ and $\boldsymbol{W}_{\boldsymbol{\omega}, x, L}(E)$, defined in (5.27) and (5.28), are measurable functions of $(\omega, E)$ for each $x \in \mathbb{R}^{d}$, and satisfy (5.32).

Theorem 7.4. Let $H_{\omega}$ be a generalized Anderson Hamiltonian on $\mathrm{L}^{2}\left(\mathbb{R}^{d}\right)$. Let $\mathcal{I} \subset \mathbb{R}$ be a bounded open interval for which there is a scale $\mathcal{L}_{3}$ such that for all $L \geq \mathcal{L}_{3}$ and $x \in \mathbb{R}^{d}$ there exists an event $\mathcal{U}_{L, x}$ as in Theorem 6.1. Then the following holds for all open intervals $I \subset \bar{I} \subset \mathcal{I}$ :

(i) For all $x, y \in \mathbb{R}^{d}$ we have

$$
\mathbb{E}\left\{\left\|\boldsymbol{W}_{\boldsymbol{\omega}, x}(E) \boldsymbol{W}_{\boldsymbol{\omega}, y}(E)\right\|_{\mathrm{L}^{\infty}\left(I, \mathrm{~d} \mu_{\boldsymbol{\omega}}(E)\right)}\right\} \leq C\langle x-y\rangle^{-\tilde{p} d},
$$

with a constant $C=C_{d, \tilde{p}, \vartheta, M, v, \mathcal{L}_{3}}$.

(ii) For all $x_{0} \in \mathbb{R}^{d}, L \geq 1$, and $\left.s \in\right] 0, \tilde{p} d / v[$ we have

$$
\mathbb{E}\left\{\left\|\boldsymbol{W}_{\boldsymbol{\omega}, x_{0}}(E) \boldsymbol{W}_{\boldsymbol{\omega}, x_{0}, L}(E)\right\|_{\mathrm{L}^{\infty}\left(I, \mathrm{~d} \mu_{\boldsymbol{\omega}}(E)\right)}^{S}\right\} \leq C L^{-(\tilde{p} d-s v)},
$$

with a constant $C=C_{d, \tilde{p}, \vartheta, M, v, \mathcal{L}_{3}, s}$.

(iii) For all $\left.x_{0} \in \mathbb{R}^{d}, s \in\right] 0, \tilde{p} d / \nu[$ and $r \in[0, \tilde{p} d-s v[$ we have, for $\mathbb{P}$-a.e. $\omega$,

$$
\left\|\boldsymbol{W}_{\boldsymbol{\omega}, x_{0}}(E) \boldsymbol{W}_{\boldsymbol{\omega}, x_{0}, 2^{k-1}}(E)\right\|_{\mathrm{L}^{\infty}\left(I, \mathrm{~d} \mu_{\boldsymbol{\omega}}(E)\right)} \leq C_{\boldsymbol{\omega}, I, s, r} 2^{-k r / s} \quad \text { for } k=0,1, \ldots
$$

As a consequence, $H_{\omega}$ has pure point spectrum in the interval I.

Remark 7.5. (ii) and (iii) hold for any $s \in] 0,2 \widetilde{p}$ [, since in this case we can choose $v>d / 2$ such that $\tilde{p} d-s v>0$.

We set

$$
\chi_{x}^{(k)}=\chi_{x, 2^{k-1}} \quad \text { and } \quad \boldsymbol{W}_{\boldsymbol{\omega}, x}^{(k)}(E)=\boldsymbol{W}_{\boldsymbol{\omega}, x, 2^{k-1}}(E) \quad \text { for } k \in \mathbb{N} .
$$

We also set $\chi_{x}^{(0)}=\chi_{x}$ and $\boldsymbol{W}_{\boldsymbol{\omega}, x}^{(0)}(E)=\boldsymbol{W}_{\boldsymbol{\omega}, x}(E)$ for convenience. Note that

$$
1 \leq \sum_{k=0}^{\infty} \chi_{x}^{(k)} .
$$

Proof of Theorem 7.4. We take $L$ sufficiently large to ensure that $I \subset \mathcal{I}_{L}$ and we can apply Theorem 6.1. We will prove (7.42); the correlation estimate (7.41) is proved in a similar way. In this case, applying (5.32), (6.7), (5.29), (5.30), and (6.4), we have

$$
\begin{array}{r}
\mathbb{E}\left\{\left\|\boldsymbol{W}_{\boldsymbol{\omega}, x_{0}}(E) \boldsymbol{W}_{\boldsymbol{\omega}, x_{0}, L}(E)\right\|_{\mathrm{L}^{\infty}\left(I, \mathrm{~d} \mu_{\boldsymbol{\omega}}(E)\right)}^{s} \leq \mathrm{e}^{-\frac{s}{2} M L^{\vartheta}} \mathbb{P}\{\Omega\}+2^{s v} L^{s v} \mathbb{P}\left\{\Omega \backslash \mathcal{U}_{L, x_{0}}\right\}\right. \\
\leq \mathrm{e}^{-\frac{s}{2} M L^{\vartheta}}+2^{s v} L^{s v} L^{-\tilde{p} d} \leq\left(1+2^{\tilde{p} d}\right) L^{-(\widetilde{p} d-s v)}
\end{array}
$$

Using the bounds (5.29) and (5.30) we get (7.42) for all $L \geq 1$. 
Given $r \in[0, \tilde{p} d-s v[$, it follows from (7.42) that

$$
\mathbb{E}\left\{\left\|\sum_{k=0}^{\infty} 2^{k r}\left[\boldsymbol{W}_{\boldsymbol{\omega}, x_{0}}(E) \boldsymbol{W}_{\boldsymbol{\omega}, x_{0}}^{(k)}(E)\right]^{s}\right\|_{\mathrm{L}^{\infty}\left(I, \mathrm{~d} \mu_{\boldsymbol{\omega}}(E)\right)}\right\} \leq C_{d, \nu, p, s, r}<\infty,
$$

and (7.43) is an immediate consequence of (7.47) using the Borel-Cantelli Lemma. Given $\omega$ for which (7.43) holds and $\phi \in \mathcal{H}_{+}$, it follows, using (5.27) and (5.28), that for $\mu_{\omega}$-a.e. $E \in I$ we have

$$
\begin{aligned}
\left\|\chi_{x_{0}} \boldsymbol{P}_{\omega}(E) \phi\right\|\left\|\boldsymbol{P}_{\omega}(E) \phi\right\| & \leq \sum_{k=0}^{\infty}\left\{\left\|\chi_{x_{0}} \boldsymbol{P}_{\omega}(E) \phi\right\|\left\|\chi_{x_{0}, k} \boldsymbol{P}_{\omega}(E) \phi\right\|\right\} \\
& \leq C_{\omega, I, s, r}\left(1-2^{-r / s}\right)^{-1}\left\|T_{x_{0}}^{-1} \boldsymbol{P}_{\omega}(E) \phi\right\|^{2}<\infty .
\end{aligned}
$$

If $\boldsymbol{P}_{\omega}(E) \phi \neq 0$, we have $\left\|\chi_{x_{0}} \boldsymbol{P}_{\omega}(E) \phi\right\| \neq 0$ for some $x_{0} \in \mathbb{R}^{d}$, and hence $\left\|\boldsymbol{P}_{\omega}(E) \phi\right\|$ $<\infty$ by (7.48), so we conclude that $\boldsymbol{P}_{\omega}(E) \phi \in \mathcal{H}=\mathrm{L}^{2}\left(\mathbb{R}^{d}\right)$. Thus we have (5.26) with $B=I$, and we conclude that $H_{\omega}$ has pure point spectrum in $I$.

Since $H_{\omega}$, as in Theorem 7.4, has pure point spectrum in the interval $\mathcal{I}$ with probability one, we might as well work with eigenfunctions, not generalized eigenfunctions. We use the notation given in (5.38).

Corollary 7.6. Let $H_{\omega}$ be a generalized Anderson Hamiltonian satisfying the hypotheses of Theorem 7.4 in a bounded open interval $\mathcal{I}$. Let $I \subset \bar{I} \subset \mathcal{I}$ be an open interval and $s \in] 0, \tilde{p} d / \nu[$. Then

(i) For all $x_{0} \in \mathbb{R}^{d}$ and $L \geq 1$ we have

$$
\mathbb{E}\left\{\sup _{E \in I}\left\|\chi_{x_{0}, L} P_{\omega}(E) \chi_{x_{0}}\right\|_{1}^{s}\right\} \leq C_{1} \mathbb{E}\left\{\sup _{E \in I}\left\|\chi_{x_{0}, L} P_{\omega, x_{0}}(E) \chi_{x_{0}}\right\|_{1}^{s}\right\} \leq C_{2} L^{-(\tilde{p} d-s v)},
$$

with $C_{1}=C_{1, d, v,\left\|V_{\text {per }}^{-}\right\|, I, s}$ and $C_{2}=C_{2, d,\left\|V_{\text {per }}^{-}\right\|, \widetilde{p}, \vartheta, M, \nu, \mathcal{L}_{3}, I, s}$.

(ii) We have

$$
\mathbb{E}\left\{\sup _{E \in I}\left(\left\|\chi_{x_{0}} P_{\omega}(E)\right\|_{2}^{2} \operatorname{tr} P_{\omega}(E)\right)^{s / 2}\right\}<\infty,
$$

and hence for $\mathbb{P}$-a.e. $\omega$ the eigenvalues of $H_{\omega}$ in I are of finite multiplicity.

Proof. Recalling (5.35) and (5.36), we have

$$
\begin{aligned}
\left\|\chi_{x_{0}, L} P_{\boldsymbol{\omega}}(E) \chi_{x_{0}}\right\|_{1} & \leq\left\|\chi_{x_{0}} P_{\boldsymbol{\omega}}(E)\right\|_{2}\left\|\chi_{x_{0}, L} P_{\boldsymbol{\omega}}(E)\right\|_{2} \\
& \leq \mu_{\boldsymbol{\omega}, x_{0}}(E) \boldsymbol{W}_{\boldsymbol{\omega}, x_{0}}(E) \boldsymbol{W}_{\boldsymbol{\omega}, x_{0}, L}(E),
\end{aligned}
$$

and (7.49) follows from (7.42) and (5.20).

In addition, we have

$$
\begin{aligned}
\left(\left\|\chi_{x_{0}} P_{\boldsymbol{\omega}}(E)\right\|_{2}^{2}\left(\operatorname{tr} P_{\boldsymbol{\omega}}(E)\right)\right)^{s / 2} & \leq \sum_{k=0}^{\infty}\left\{\left\|\chi_{x_{0}} P_{\boldsymbol{\omega}}(E)\right\|_{2}\left\|\chi_{x_{0}}^{(k)} P_{\boldsymbol{\omega}}(E)\right\|_{2}\right\}^{s} \\
& \leq\left\{\sum_{k=0}^{\infty}\left\{\boldsymbol{W}_{\boldsymbol{\omega}, x_{0}}(E) \boldsymbol{W}_{\boldsymbol{\omega}, x_{0}}^{(k)}(E)\right\}^{s}\right\}\left\{\mu_{\boldsymbol{\omega}, x_{0}}(I)\right\}^{s}
\end{aligned}
$$

so (7.50) follows from (7.42) and (5.20). 
Since for $\mathbb{P}$-a.e. $\omega$ the operator $H_{\omega}$ has pure point spectrum in the interval $I$, it follows from (7.50) that for $\mathbb{P}$-a.e. $\omega$ we have

$$
\left\|\chi_{x_{0}} P_{\omega}(E)\right\|_{2}^{2} \operatorname{tr} P_{\omega}(E)<\infty \quad \text { for all } E \in I,
$$

and hence, since $\chi_{x_{0}} P_{\omega}(E) \neq 0$ for some $x_{0} \in \mathbb{R}^{d}$ if $P_{\omega}(E) \neq 0$, we have $\operatorname{tr} P_{\omega}(E)<\infty$ for all $E \in I$.

We can now prove dynamical localization in expectation.

Corollary 7.7. Let $H_{\omega}$ be a generalized Anderson Hamiltonian satisfying the hypotheses of Theorem 7.4 in a bounded open interval $\mathcal{I}$. The following holds for all $x_{0} \in \mathbb{R}^{d}$ and open intervals $I \subset \bar{I} \subset \mathcal{I}$ :

(i) For all $L \geq 1$ and $s \in] 0, \tilde{p} d / v[$ we have

$$
\begin{array}{r}
\mathbb{E}\left\{\sup _{f \in \mathcal{B}_{b, 1}}\left\|\chi_{x_{0}, L} f\left(H_{\omega}\right) P_{\omega}(I) \chi_{x_{0}}\right\|_{1}^{s}\right\} \\
\mathbb{E}\left\{\sup _{E \in I}\left\|\chi_{x_{0}, L} P_{\omega}^{(E)} \chi_{x_{0}}\right\|_{1}^{s}\right\} \leq C L^{-(\tilde{p} d-s v),},
\end{array}
$$

with $C=C_{d,\left\|V_{\text {per }}^{-}\right\|, \tilde{p}, \vartheta, M, v, \mathcal{L}_{3}, I, s}$.

(ii) Given $b>0$, for all $s \in] 0, \widetilde{p} /(b+1 / 2)[$ we have

$$
\begin{array}{r}
\mathbb{E}\left\{\sup _{f \in \mathcal{B}_{b, 1}}\left\|\left\langle X-x_{0}\right\rangle^{b d} f\left(H_{\omega}\right) P_{\omega}(I) \chi_{x_{0}}\right\|_{1}^{s}\right\} \leq C<\infty, \\
\mathbb{E}\left\{\sup _{t \in \mathbb{R}}\left\|\left\langle X-x_{0}\right\rangle^{b d} \mathrm{e}^{-i t H_{\omega}} P_{\omega}(I) \chi_{x_{0}}\right\|_{1}^{s}\right\} \leq C<\infty, \\
\mathbb{E}\left\{\sup _{E \in I}\left\|\left\langle X-x_{0}\right\rangle^{b d} P_{\omega}^{(E)} \chi_{x_{0}}\right\|_{1}^{s}\right\} \leq C<\infty,
\end{array}
$$

with $C=C_{d,\left\|V_{\text {per }}^{-}\right\|, \tilde{p}, \vartheta, M, v, \mathcal{L}_{3}, I, b, s}$.

Proof. Given $f \in \mathcal{B}_{b, 1}$, it follows from (5.37) that

$$
\begin{aligned}
\left\|\chi_{x_{0}, L} f\left(H_{\omega}\right) P_{\omega}(I) \chi_{x_{0}}\right\|_{1} & \leq \int_{I}|f(E)|\left\|\chi_{x_{0}, L} P_{\boldsymbol{\omega}, x_{0}}(E) \chi_{x_{0}}\right\|_{1} \mathrm{~d} \mu_{\boldsymbol{\omega}, x_{0}}(E) \\
& \leq \sup _{E \in I}\left\|\chi_{x_{0}, L} P_{\boldsymbol{\omega}, x_{0}}(E) \chi_{x_{0}}\right\|_{1} \mu_{\boldsymbol{\omega}, x_{0}}(I),
\end{aligned}
$$

and hence (7.54) is an immediate consequence of (7.49).

The estimate (7.55) is proven similarly to (7.32). We introduce the decomposition $P_{\omega}^{(E)}=g^{2}\left(H_{\omega}\right)+f_{E}\left(H_{\omega}\right)$ as in (7.35), and (7.55) follows from (7.35), (7.54), and (7.40). Since

Given $b>0$ and $s \in] 0, \tilde{p} /(b+1 / 2)$ [, we pick $v>d / 2$ such that $s \in] 0, \tilde{p} /(b+v / d)[$.

$$
\left\|\left\langle X-x_{0}\right\rangle^{b d} f\left(H_{\omega}\right) P_{\omega}(I) \chi_{x_{0}}\right\|_{1} \leq C_{d, b} \sum_{k=0}^{\infty} 2^{k b d}\left\|\chi_{x_{0}}^{(k)} f\left(H_{\omega}\right) P_{\omega}(I) \chi_{x_{0}}\right\|_{1},
$$

the estimate (7.56) follows from (7.54); (7.57) is a special case of (7.56). Similarly, (7.58) follows from (7.55). 


\section{Log-Hölder continuity of the integrated density of states}

We will now assume that the conclusions of the multiscale analysis (i.e., of Proposition 4.6) hold for all energies in a bounded open interval $\mathcal{I}$, and prove log-Hölder continuity of the integrated density of states.

Given a generalized Anderson Hamiltonian $H_{\omega}$ and $x_{0} \in \mathbb{R}^{d}$, we set

$$
N_{x_{0}}(E)=\mathbb{E} \operatorname{tr}\left\{\chi_{x_{0}} P_{\omega}^{(E)} \chi_{x_{0}}\right\} \quad \text { for } E \in \mathbb{R} .
$$

Theorem 8.1. Let $H_{\omega}$ be a generalized Anderson Hamiltonian on $\mathrm{L}^{2}\left(\mathbb{R}^{d}\right)$. Consider a bounded open interval $\mathcal{I} \subset \mathbb{R}, m>0, p>0$, and $\varsigma \in] 0,1$, and assume there is a scale $\mathcal{L}$ such that all scales $L \geq \mathcal{L}$ are $(E, m, \varsigma, p)$-good for all energies $E \in \mathcal{I}$. Then, for all $0<\widehat{p}<p$, closed interval $I \subset \mathcal{I}$ with length $|I| \leq 1 / 2$, and $x_{0} \in \mathbb{R}^{d}$, we have

$$
\left|N_{x_{0}}\left(E_{2}\right)-N_{x_{0}}\left(E_{1}\right)\right| \leq \frac{C_{\widehat{p}, I}}{|\log | E_{2}-E_{1}||^{\widehat{p} d}} \quad \text { for all } E_{1}, E_{2} \in I .
$$

The proof of this theorem will use the Helffer-Sjöstrand formula (see [Dav, Section 2.2] and [HuS, Appendix B] for details). Given $g \in C^{\infty}(\mathbb{R}), n \in \mathbb{N}$, and $a>0$, we define a quasi-analytic extension of $g$ of order $n$ by

$$
\tilde{g}_{n, a}(z):=\left\{\sum_{r=0}^{n} \frac{1}{r !} g^{(r)}(u)(i v)^{r}\right\} \xi\left(\frac{a v}{\langle u\rangle}\right),
$$

where $z=u+i v,\langle u\rangle=\left(1+|u|^{2}\right)^{1 / 2}$, and $\xi \in C^{\infty}(\mathbb{R})$ is such that $0 \leq \xi \leq 1$, $\xi(u)=1$ if $|u| \leq 1, \xi(u)=0$ if $|u| \geq 2$. (We choose and fix $\xi$.) We set $\mathrm{d} \tilde{g}_{n, a}(z):=$ $(2 \pi)^{-1} \partial_{\bar{z}} \tilde{g}_{n, a}(z) \mathrm{d} u \mathrm{~d} v$, with $\partial_{\bar{z}}=\partial_{u}+i \partial_{v}$, and $\left|\mathrm{d} \tilde{g}_{n, a}(z)\right|:=(2 \pi)^{-1}\left|\partial_{\bar{z}} \tilde{g}_{n, a}(z)\right| \mathrm{d} u \mathrm{~d} v$. Proceeding as in the derivation of [HuS, Eq. (B.8)], we get, for all $n \in \mathbb{N}, a>0$, and $s \in[0, n]$,

$$
\int_{\mathbb{R}^{2}}\left|\mathrm{~d} \tilde{g}_{n}(z)\right||\Im z|^{-(s+1)} \leq C_{n, s}\left\{\{g\}_{n, s, a} \leq C_{n, s} \max \left\{a^{s+1}, a^{s-n}\right\}\left\{\{g\}_{n},\right.\right.
$$

with

$$
\left\{\{g\}_{n, s, a}:=\sum_{r=0}^{n+1} a^{-(r-s-1)} \int_{\mathbb{R}} \mathrm{d} u\langle u\rangle^{r-s-1}\left|g^{(r)}(u)\right|, \quad\left\{\{g\}_{n}=\left\{\{g\}_{n, 0,1} .\right.\right.\right.
$$

In particular, if $\left\{\{g\}_{n}<\infty\right.$, then for any self-adjoint operator $K$ and $a>0$ we have

$$
g(K)=\int_{\mathbb{R}^{2}} \mathrm{~d} \tilde{g}_{n, a}(z)(K-z)^{-1},
$$

where the integral converges absolutely in operator norm.

Remark 8.2. In the usual Helffer-Sjöstrand formula there is no parameter $a$ in the definition of the quasi-analytic extension, i.e., $a=1$ in (8.3) (e.g., [Dav, HuS]). The proof of Theorem 8.1 requires the insertion of the parameter $a$ in (8.3), which is then chosen according to the scale $L$-we will need $a \approx \mathrm{e}^{L^{1-\varsigma}}$. 
Proof of Theorem 8.1. Let $\eta \in] 0, p[$ and $I \subset \mathcal{I}$ be a closed interval with length $|I| \leq$ $1 / 2$. Without loss of generality we assume $\eta>\varsigma /(1-\varsigma)$. We consider scales $L \geq \mathcal{L}$ such that $\operatorname{dist}(I, \mathbb{R} \backslash \mathcal{I})>\frac{1}{2} \mathrm{e}^{-L^{1-\varsigma}}$. Let $I_{L} \subset I$ be a closed interval of length $\left|I_{L}\right|=$ $\mathrm{e}^{-L^{1-\varsigma}}$, so it can be written as $I_{L}=\left[E-\frac{1}{2} \mathrm{e}^{-L^{1-\varsigma}}, E+\frac{1}{2} \mathrm{e}^{-L^{1-\varsigma}}\right]$ with $E \in I$. Set $\tilde{I}_{L}=\left[E-\mathrm{e}^{-L^{1-\varsigma}}, E+\mathrm{e}^{-L^{1-\varsigma}}\right] \subset \mathcal{I}$. We fix $h_{L} \in C^{\infty}(\mathbb{R}), 0 \leq h_{L} \leq 1$, such that

$$
\operatorname{supp} h_{L} \subset \tilde{I}_{L}, \quad h_{L} \chi_{I_{L}}=\chi_{I_{L}}, \quad\left|h_{L}^{(j)}\right| \leq C_{d} \mathrm{e}^{j L^{1-\varsigma}} \quad \text { for } j=1, \ldots, d+2,
$$

with $C_{d}$ a constant independent of $L$.

Given $x_{0} \in \mathbb{R}^{d}$, we let $\mathcal{Y}_{L}=\mathcal{Y}_{L, x_{0}}$ be the event that the box $\Lambda_{L}=\Lambda_{L}\left(x_{0}\right)$ is $(\omega, E, m, \varsigma, \eta)$-pgood (cf. Definition 3.15). Since all large scales $L \geq \mathcal{L}$ are $(E, m, \varsigma, p)$ good by hypothesis, we have, using (3.60) and (5.18),

$$
\begin{aligned}
\mathbb{E} \operatorname{tr}\left\{\chi_{x_{0}} P_{\omega}\left(I_{L}\right) \chi_{x_{0}}\right\} & \leq \mathbb{E} \operatorname{tr}\left\{\chi_{x_{0}} h_{L}\left(H_{\omega}\right) \chi_{x_{0}}\right\} \\
& \leq \mathbb{E}\left\{\operatorname{tr}\left\{\chi_{x_{0}} h_{L}\left(H_{\omega}\right) \chi_{x_{0}} ; \mathcal{Y}_{L}\right\}\right\}+C_{\mathcal{I}} L^{-\frac{p-\eta}{1+\eta} d},
\end{aligned}
$$

with a constant $C_{\mathcal{I}}=C_{d, v,\left\|V_{\text {per }}^{-}\right\|, \sup \mathcal{I}}$.

If $\omega \in \mathcal{Y}_{L}, \Lambda_{L}$ is $\left(\omega, E, M_{1}, \varsigma\right)$-good by Lemma 3.16 (with $M_{1}$ given in (3.56)), and hence $h_{L}\left(H_{\omega, \Lambda_{L}}\right)=0$. Thus,

$$
\operatorname{tr}\left\{\chi_{x_{0}} h_{L}\left(H_{\omega}\right) \chi_{x_{0}}\right\}=\operatorname{tr}\left\{\chi_{x_{0}} h_{L}\left(H_{\omega}\right) \chi_{x_{0}}-\chi_{x_{0}} h_{L}\left(H_{\omega, \Lambda_{L}}\right) \chi_{x_{0}}\right\} \quad \text { for } \omega \in \mathcal{Y}_{L}
$$

The right-hand-side of (8.9) may now be estimated by the Helffer-Sjöstrand formula. We apply the Helffer-Sjöstrand formula to $h_{L}\left(H_{\boldsymbol{\omega}}\right)$ and $h_{L}\left(H_{\boldsymbol{\omega}, \Lambda_{L}}\right)$, with $a \geq 1$ in (8.3) to be chosen later depending on $L$. We take $\phi_{0} \in C_{c}^{\infty}(\mathbb{R})$ such that $0 \leq \phi_{0} \leq 1, \phi_{0}=1$ on $\Lambda_{L / 2}\left(x_{0}\right)$, and supp $\phi_{0} \subset \Lambda_{L / 2+10}\left(x_{0}\right)$. We have, with $n \in \mathbb{N}$ to be chosen later (we omit $n$ and $a$ from the notation),

$$
\begin{aligned}
T_{\omega}^{L} & =T_{\omega}^{L, x_{0}}:=\chi_{x_{0}} h_{L}\left(H_{\omega}\right) \chi_{x_{0}}-\chi_{x_{0}} h_{L}\left(H_{\boldsymbol{\omega}, \Lambda_{L}}\right) \chi_{x_{0}} \\
& =\int_{\mathbb{R}^{2}} \mathrm{~d} \widetilde{h_{L}}(z)\left\{\chi_{x_{0}} R_{\boldsymbol{\omega}}(z) \chi_{x_{0}}-\chi_{x_{0}} R_{\boldsymbol{\omega}, \Lambda_{L}}(z) \chi_{x_{0}}\right\} \\
& =\int_{\mathbb{R}^{2}} \mathrm{~d} \widetilde{h_{L}}(z)\left\{\chi_{x_{0}} R_{\boldsymbol{\omega}}(z) \phi_{0} \chi_{x_{0}}-\chi_{x_{0}} \phi_{0} R_{\boldsymbol{\omega}, \Lambda_{L}}(z) \chi_{x_{0}}\right\} \\
& =\int_{\mathbb{R}^{2}} \mathrm{~d} \widetilde{h_{L}}(z)\left\{\chi_{x_{0}} R_{\boldsymbol{\omega}}(z) W\left(\phi_{0}\right) R_{\boldsymbol{\omega}, \Lambda_{L}}(z) \chi_{x_{0}}\right\}
\end{aligned}
$$

where we used the geometric resolvent identity as in (2.36).

We now pick functions $\phi_{i} \in C_{c}^{\infty}(\mathbb{R}), i=1, \ldots, 2 k-1$, where $k \in \mathbb{N}$ will be chosen later, such that $0 \leq \phi_{i} \leq 1, \phi_{i}=1$ on supp $\nabla \phi_{i-1}$, and supp $\phi_{i} \subset \Lambda_{L / 2+50, L / 2-50}\left(x_{0}\right)$. Using the resolvent identity $2 k-1$ times, noticing $\phi_{i} \chi_{x_{0}}=0$ for $i=1, \ldots, 2 k-1$, and writing $\chi_{\nabla \phi}=\chi_{\text {supp } \nabla \phi}$, we get

$$
\begin{aligned}
\chi_{x_{0}} R_{\omega}(z) W\left(\phi_{0}\right)=\chi_{x_{0}} R_{\omega}(z) W\left(\phi_{2 k-1}\right) R_{\omega}(z) W\left(\phi_{2 k-2}\right) \ldots R_{\omega}(z) W\left(\phi_{1}\right) R_{\omega}(z) W\left(\phi_{0}\right) \\
=\left\{\chi_{x_{0}} R_{\omega}(z)\right\}\left\{W\left(\phi_{2 k-1}\right) R_{\omega}(z) W\left(\phi_{2 k-2}\right)\right\}\left\{\chi_{\nabla \phi_{2 k-2}} R_{\omega}\right\} \\
\quad \times\left\{W\left(\phi_{2 k-3}\right) R_{\omega}(z) W\left(\phi_{2 k-4}\right)\right\} \ldots\left\{\chi_{\nabla \phi_{2}} R_{\omega}(z)\right\}\left\{W\left(\phi_{1}\right) R_{\omega}(z) W\left(\phi_{0}\right)\right\} .
\end{aligned}
$$


Given $\phi \in C_{c}^{\infty}(\mathbb{R})$, it follows from (2.24) that for all $\omega \in \Omega$,

$$
\left\|\left(H_{\omega}+1\right)^{-1 / 2} W(\phi)\right\|=\left\|W(\phi)\left(H_{\omega}+1\right)^{-1 / 2}\right\| \leq C_{\phi}:=C_{1}\left(\|\Delta \phi\|_{\infty}+\|\nabla \phi\|_{\infty}\right),
$$

where $C_{1}=C_{d,\left\|V_{\text {per }}^{-}\right\|}$. Moreover, for all $x \in \mathbb{R}^{d}$ we have

$$
\left\|\chi_{x}\left(H_{\omega}+1\right)^{-1}\right\|_{k_{d}} \leq C_{2}<\infty \quad \text { with } \quad k_{d}=[d / 2]+1
$$

the constant $C_{2}=C_{d,\left\|V_{\text {per }}\right\|, U_{+}}$being independent of $x$ (cf. [KlKS, Eqs. (130)-(136)]). We have

$$
\left\|\left(H_{\omega}+1\right) R_{\omega}(z)\right\| \leq 1+\frac{1+|z|}{|\Im z|} \leq 2+\frac{1+|\Re z|}{|\Im z|},
$$

Using (8.12)-(8.14), we have

$$
\left\|W\left(\phi_{i}\right) R_{\omega}(z) W\left(\phi_{i-1}\right)\right\| \leq C_{\phi_{i}} C_{\phi_{i-1}}\left(2+\frac{1+|\Re z|}{|\Im z|}\right)
$$

and, for all measurable sets $\Xi \subset \Lambda_{L}$, we get

$$
\left\|\chi_{\Xi} R_{\omega}(z)\right\|_{k_{d}} \leq C_{2}\left(2+\frac{1+|\Re z|}{|\Im z|}\right) L^{d} .
$$

We now take $k=k_{d}$ as in (8.13), and note that we can choose the functions $\phi_{i}$ $\in C_{c}^{\infty}(\mathbb{R}), i=0,1, \ldots, 2 k_{d}-1$, so that $C_{\phi_{i}} \leq C_{3}=C_{d,\left\|V_{\text {per }}^{-}\right\|}$, a constant independent of $\Lambda_{L}$. From (8.11), (8.15) and (8.16), we get, for all $\omega \in \Omega$,

$$
\begin{aligned}
& \left\|\chi_{x_{0}} R_{\omega}(z) W\left(\phi_{0}\right) R_{\omega, \Lambda_{L}}(z) \chi_{x_{0}}\right\|_{1} \\
& \leq C_{4} L^{d k_{d}}\left(2+\frac{1+|\Re z|}{|\mathfrak{\Im} z|}\right)^{2 k_{d}}\left\|\chi_{\nabla \phi_{0}} R_{\omega, \Lambda_{L}}(z) \chi_{x_{0}}\right\|,
\end{aligned}
$$

with a constant $C_{4}=\left(C_{2} C_{3}^{2}\right)^{k d}=C_{d,\left\|V_{\text {per }}\right\|, U_{+}}$.

We can now estimate $\operatorname{tr} T_{\omega}^{L}$. First, note that, with $\mathcal{I}_{+}=\sup \mathcal{I}<\infty$,

$$
\operatorname{supp} \widetilde{h_{L}} \subset\left\{z=u+i v ; u \in \widetilde{I}_{L},|v| \leq \frac{2}{a}\langle u\rangle\right\} \subset \widetilde{I}_{L}+i\left[-\frac{2}{a}\left\langle\mathcal{I}_{+}\right\rangle, \frac{2}{a}\left\langle\mathcal{I}_{+}\right\rangle\right]
$$

and hence (recall $a \geq 1$ )

$$
2+\frac{1+|\Re z|}{|\Im z|} \leq \frac{\frac{4}{a}\left\langle\mathcal{I}_{+}\right\rangle+1+\mathcal{I}_{+}}{|\mathfrak{I} z|} \leq \frac{C_{\mathcal{I}_{+}}}{|\mathfrak{I} z|} \quad \text { for all } z \in \operatorname{supp} \widetilde{h_{L}}
$$


with $C_{\mathcal{I}_{+}}=5\left(1+\mathcal{I}_{+}\right)$. Combining (8.10), (8.17), (8.19), and (8.4), and using the fact that $\{\{g\}\}_{n}$ in (8.5) is increasing in $n$, we get

$$
\begin{aligned}
\left|\operatorname{tr} T_{\omega}^{L}\right| & \leq \int_{\mathbb{R}^{2}}\left|\mathrm{~d} \widetilde{h_{L}}(z)\right|\left\|\chi_{x_{0}} R_{\boldsymbol{\omega}}(z) W\left(\phi_{0}\right) R_{\boldsymbol{\omega}, \Lambda_{L}}(z) \chi_{x_{0}}\right\|_{1} \\
& \leq C_{4} C_{\mathcal{I}_{+}}^{2 k_{d}} L^{d k_{d}} \int_{\mathbb{R}^{2}}\left|\mathrm{~d} \widetilde{h_{L}}(z)\right||\Im z|^{-2 k_{d}}\left\|\chi_{\nabla \phi_{0}} R_{\boldsymbol{\omega}, \Lambda_{L}}(z) \chi_{x_{0}}\right\| \\
& \leq C_{4} C_{\mathcal{I}_{+}}^{2 k_{d}} L^{d k_{d}} a^{2 k_{d}}\left\{\left\{h_{L}\right\}\right\}_{2 k_{d}-1}\left\{\max _{z \in \operatorname{supp} \widetilde{h_{L}}}\left\|\chi \nabla \phi_{0} R_{\boldsymbol{\omega}, \Lambda_{L}}(z) \chi_{x_{0}}\right\|\right\} \\
& \leq C_{4} C_{d} C_{\mathcal{I}_{+}}^{d+2} L^{\frac{d}{2}(d+2)} a^{d+2}\left\{\left\{h_{L}\right\}\right\}_{d+1}\left\{\max _{z \in \operatorname{supp} \widetilde{h_{L}}}\left\|\chi_{\nabla \phi_{0}} R_{\boldsymbol{\omega}, \Lambda_{L}}(z) \chi_{x_{0}}\right\|\right\} .
\end{aligned}
$$

In view of (8.7) and (8.5), we have

$$
\left\{\left\{h_{L}\right\}_{d+1} \leq C_{d, \mathcal{I}_{+}}\left|\tilde{I}_{L}\right| \mathrm{e}^{(d+2) L^{1-\varsigma}}=2 C_{d, \mathcal{I}_{+}} \mathrm{e}^{(d+1) L^{1-\varsigma}} \quad \text { for all } \omega \in \Omega .\right.
$$

We are now ready to estimate the quantity in (8.9). We choose $a=2\left\langle\mathcal{I}_{+}\right\rangle \mathrm{e}^{L^{1-\varsigma}}$, so it follows from (8.18) that

$$
\operatorname{supp}_{z \in \operatorname{supp} \widetilde{h_{L}}}|z-E| \leq \mathrm{e}^{-L^{1-\varsigma}}+\frac{2}{a}\left\langle\mathcal{I}_{+}\right\rangle \leq 2 \mathrm{e}^{-L^{1-\varsigma}} .
$$

Since $\eta>\varsigma /(1-\varsigma)$, we may take $L$ large enough to ensure $2 \mathrm{e}^{-L^{1-\varsigma}}<\mathrm{e}^{-m L^{1 /(1+\eta)}}$, so Lemma 3.16 guarantees that, for large $L$, for all $\omega \in \mathcal{Y}_{L}$ the box $\Lambda_{L}$ is $(\omega, z, m / 2, \varsigma)$ good for all $z \in \operatorname{supp} \widetilde{h_{L}}$. Thus, for large $L$,

$$
\max _{z \in \operatorname{supp} \widetilde{h_{L}}}\left\|\chi_{\nabla \phi_{0}} R_{\boldsymbol{\omega}, \Lambda_{L}}(z) \chi_{x_{0}}\right\| \leq(L / 2+11)^{d} \mathrm{e}^{-\frac{m}{2} \frac{L}{4}} \leq \mathrm{e}^{-\frac{m}{10} L} .
$$

It follows from (8.9), (8.10), (8.20), and (8.23) that for all $\omega \in \mathcal{Y}_{L}$ we have, again taking $L$ large,

$$
\begin{aligned}
\operatorname{tr}\left\{\chi_{x_{0}} h_{L}\left(H_{\omega}\right) \chi_{x_{0}}\right\} & \leq C_{4} C_{d} C_{\mathcal{I}_{+}}^{d+2} L^{\frac{d}{2}(d+2)}\left(2 C_{d, \mathcal{I}_{+}} \mathrm{e}^{(d+1) L^{1-\varsigma}}\right)\left(2\left\langle\mathcal{I}_{+}\right\rangle \mathrm{e}^{L^{1-\varsigma}}\right)^{d+2} \mathrm{e}^{-\frac{m}{10} L} \\
& \leq \mathrm{e}^{-\frac{m}{20} L} .
\end{aligned}
$$

Combining (8.8) and (8.24), we get, for large $L$,

$$
\mathbb{E} \operatorname{tr}\left\{\chi_{x_{0}} P_{\omega}\left(I_{L}\right) \chi_{x_{0}}\right\} \leq \mathrm{e}^{-\frac{m}{20} L}+C_{\mathcal{I}} L^{-\frac{p-\eta}{1+\eta} d} \leq 2 C_{\mathcal{I}} L^{-\frac{p-\eta}{1+\eta} d} .
$$

In particular, for all intervals $J \subset I$ with sufficiently small length $|J|$, we have

$$
\mathbb{E} \operatorname{tr}\left\{\chi_{x_{0}} P_{\omega}(J) \chi_{x_{0}}\right\} \leq 2 C_{\mathcal{I}}|\log | J||^{-\frac{p-\eta}{(1+\eta)(1-\varsigma)} d} .
$$

The estimate (8.2) follows.

Remark 8.3. The proof of Theorem 8.1 uses the pgood boxes of Definition 3.15 because we need Lemma 3.16. It does not suffice to use good boxes. 


\section{Appendix. A quantitative unique continuation principle for Schrödinger operators}

In this appendix we rewrite Bourgain and Kenig's quantitative unique continuation principle for Schrödinger operators [BoK] in a convenient form for our purposes. We also give an application of this quantitative unique continuation principle to periodic Schrödinger operators, giving an alternative proof to Combes, Hislop and Klopp's lower bound estimate for spectral projections [CoHK1].

We use the norm $|x|:=\left(\sum_{j=1}^{d}\left|x_{j}\right|^{2}\right)^{1 / 2}$ for $x=\left(x_{1}, \ldots, x_{d}\right) \in \mathbb{R}^{d}$; all distances in $\mathbb{R}^{d}$ will be measured with respect to this norm. Given $x \in \mathbb{R}^{d}$ and $\delta>0$, we set $B(x, \delta):=\left\{y \in \mathbb{R}^{d} ;|y-x|<\delta\right\}$ and $B(x, \delta)^{*}:=B((x, \delta) \backslash\{x\}$. Given subsets $A$ and $B$ of $\mathbb{R}^{d}$, and a function $\varphi$ on set $B$, we set $\varphi_{A}:=\varphi \chi_{A \cap B}$. In particular, given $x \in \mathbb{R}^{d}$ and $\delta>0$ we write $\varphi_{x, \delta}:=\varphi_{B(x, \delta / 2) \text {. }}$

We also set

$$
C_{1}=\mathrm{e}^{\int_{0}^{1} \frac{1-\mathrm{e}^{-t}}{t} \mathrm{~d} t} ; \quad \text { note } \quad 2<\mathrm{e}^{3 / 4}<C_{1}<\mathrm{e}<3
$$

\section{A.1. The quantitative unique continuation principle}

The following theorem is our version of [BoK, Lemma 3.10].

Theorem A.1. Let $G$ be an open subset of $\mathbb{R}^{d}$. Let $\psi \in \mathrm{H}^{2}(G)$ and $\zeta \in \mathrm{L}^{2}(G)$ be real-valued functions satisfying

$$
-\Delta \psi+V \psi=\zeta \quad \text { a.e. on } G,
$$

where $V$ is a real measurable function on $G$ with $\|V\|_{\infty} \leq K<\infty$. Fix $\delta, D_{0}, D$ such that $0<\delta / 4 \leq D_{0} \leq D$. There exists a constant $m=m\left(d, \delta, D_{0}\right)>0$ such that, given a measurable set $\Theta \subset G$ with $\operatorname{diam} \Theta \leq D$, and $x \in G$ such that

$$
R:=\operatorname{dist}(x, \Theta) \geq D \quad \text { and } \quad B\left(x, 4 C_{1} R+2 D_{0}\right) \subset G,
$$

where $C_{1}$ is the constant in (A.1), we have

$$
(1+K)\left\|\psi_{x, \delta}\right\|_{2}^{2}+\left\|\zeta_{G}\right\|_{2}^{2} \geq R^{-m\left(1+K^{2 / 3}+\log \left(\left\|\psi_{G}\right\|_{2}\left\|\psi_{\Theta}\right\|_{2}^{-1}\right)\right) R^{4 / 3}}\left\|\psi_{\Theta}\right\|_{2}^{2} .
$$

If the open set $G$ is bounded, the second condition in (A.3) restricts the application of Theorem A.1 to sites $x \in G$ sufficiently far away from the boundary of $G$. When $G$ is a box $\Lambda$, and (A.2) holds on $\Lambda$ with either a Dirichlet or a periodic boundary condition, Theorem A.1 can be extended to sites $x \in \Lambda$ near the boundary of $\Lambda$ as in the following corollary.

Corollary A.2. Consider the Schrödinger operator $H_{\Lambda}:=-\Delta_{\Lambda}+V$ on $\mathrm{L}^{2}(\Lambda)$, where $\left.\Lambda=\Lambda_{L}\left(x_{0}\right)=x_{0}+\right]-L / 2, L / 2\left[^{d}\right.$, the open box of side $L>0$ centered at $x_{0} \in \mathbb{R}^{d}$, $\Delta_{\Lambda}$ is the Laplacian with either a Dirichlet or a periodic boundary condition on $\Lambda$, and $V$ is a bounded potential on $\Lambda$ with $\|V\|_{\infty} \leq K<\infty$. Let $\psi \in \mathcal{D}\left(\Delta_{\Lambda}\right)$. 
(i) Fix $\delta, D$ such that $0<\delta / 4 \leq D$, There exists a constant $\tilde{m}=\tilde{m}(d, \delta, D)>0$ such that, given a measurable set $\Theta \subset \Lambda$ with diam $\Theta \leq D$, and $x \in \Lambda$ such that

$$
B(x, \delta / 2) \subset \Lambda \quad \text { and } \quad R:=\operatorname{dist}(x, \Theta) \geq D,
$$

we have

$$
(1+K)\left\|\psi_{x, \delta}\right\|_{2}^{2}+(29 \sqrt{d})^{d}\left\|\left(H_{\Lambda} \psi\right)_{\Lambda}\right\|_{2}^{2} \geq R^{-\widetilde{m}\left(1+K^{2 / 3}+\log \left(\left\|\psi_{\Lambda}\right\|_{2}\left\|\psi_{\Theta}\right\|_{2}^{-1}\right)\right) R^{4 / 3}}\left\|\psi_{\Theta}\right\|_{2}^{2} .
$$

(ii) Let $L \geq 2$ and $0<\delta \leq L$. Then there exists a constant $\widehat{m}=\widehat{m}(d, \delta)>0$ such that for all $x \in \Lambda$ with $B(x, \delta / 2) \subset \Lambda$ we have

$$
(1+K)\left\|\psi_{x, \delta}\right\|_{2}^{2}+(41)^{d}\left\|\left(H_{\Lambda} \psi\right)_{\Lambda}\right\|_{2}^{2} \geq L^{-\widehat{m}\left(1+K^{2 / 3}\right) L^{4 / 3}}\left\|\psi_{\Lambda}\right\|_{2}^{2} .
$$

We will prove Theorem A.1 from Bourgain and Kenig's Carleman-type inequality estimate [BoK, Lemma 3.15], which we state in the next lemma.

Lemma A.3. Consider the function $w(x)=\varphi(|x|)$ on $\mathbb{R}^{d}$, where

$$
\varphi(s):=\mathrm{e}^{-\int_{0}^{s} \frac{1-\mathrm{e}^{-t}}{t} \mathrm{~d} t} s \quad \text { for } s \in[0, \infty[
$$

is a strictly increasing continuous function on $\left[0, \infty\left[, C^{\infty}\right.\right.$ on $] 0, \infty[$. In particular,

$$
\frac{1}{C_{1}}|x| \leq w(x) \leq|x| \quad \text { for all } x \in B(0,1),
$$

where $C_{1}$ is the constant in (A.1). Then there are positive finite constants $C_{2}$ and $C_{3}$, depending only on $d$, such that for all $\alpha \geq C_{2}$ and all real-valued functions $f \in$ $C_{\mathrm{c}}^{\infty}\left(B(0,1)^{*}\right)$ we have

$$
\alpha^{3} \int_{\mathbb{R}^{d}} w^{-1-2 \alpha} f^{2} \mathrm{~d} x \leq C_{3} \int_{\mathbb{R}^{d}} w^{2-2 \alpha}(\Delta f)^{2} \mathrm{~d} x .
$$

We refer to $[\mathrm{BoK}]$ for the proof. We shall use Lemma A.3 with a function $f$ that is not necessarily smooth, but $f \in \mathrm{H}_{\mathrm{loc}}^{2}$. However in our case $f$ is compactly supported away from zero, and thus we can use the following extension of Lemma A.3.

Lemma A.4. Let $f \in \mathrm{H}^{2}(B(0,1))$ be real-valued with supp $f \subset B(0,1)^{*}$. Then (A.10) holds for all $\alpha \geq C_{2}$.

Proof. This follows from Lemma A.3 by an approximation argument. Let $f$ be as in the lemma, pick $h \in C_{\mathrm{c}}^{\infty}(\mathbb{R})$ with $\int h(t) \mathrm{d} t=1$, and set $h_{\eta}(t):=\eta^{-d} h(t / \eta)$. Note that for $\eta$ small enough we have $f_{\eta}:=f * h_{\eta} \in C_{\mathrm{c}}^{\infty}\left(B(0,1)^{*}\right)$. Thus, for such $\eta$ 's, Lemma A.3 applies to $f_{\eta}$. Then, as $\eta$ goes to zero, $f \eta$ converges to $f$ in $\mathrm{L}^{2}\left(\mathbb{R}^{d}\right)$ and $\Delta f_{\eta}=(\Delta f) * h_{\eta}$ to $\Delta f$ in $\mathrm{L}^{2}\left(\mathbb{R}^{d}\right)$. Since $w^{-1}$ is bounded above and below on $B(0, R) \backslash B(0, \delta)$ for any $\delta>0$, the lemma follows.

We now rewrite these lemmas as follows. 
Lemma A.5. Given $\varrho>0$, there exists a function $w_{\varrho}(x)=\varphi_{\varrho}(|x|)$ on $\mathbb{R}^{d}$, where $\varphi_{\varrho}$ is a strictly increasing continuous real valued function on $\left[0, \infty\left[, C^{\infty}\right.\right.$ on $] 0, \infty[$, such that

$$
\frac{1}{C_{1} \varrho}|x| \leq w_{\varrho}(x) \leq \frac{1}{\varrho}|x| \quad \text { for all } x \in B(0, \varrho),
$$

and for all $\alpha \geq C_{2}$ and all real-valued functions $f \in \mathrm{H}^{2}(B(0, \varrho))$ with supp $f \subset$ $B(0, \varrho)^{*}$ we have

$$
\alpha^{3} \int_{\mathbb{R}^{d}} w_{\varrho}^{-1-2 \alpha} f^{2} \mathrm{~d} x \leq C_{3} \varrho^{4} \int_{\mathbb{R}^{d}} w_{\varrho}^{2-2 \alpha}(\Delta f)^{2} \mathrm{~d} x,
$$

where $C_{1}, C_{2}, C_{3}$ are the constants of Lemma A.3.

Proof. (A.12) follows from (A.10) by a change of variables, with $w_{\varrho}(x)=w(x / \varrho)$.

We are ready to prove Theorem A.1 and Corollary A.2.

Proof of Theorem A.1. Without loss of generality we assume

$$
\left\|\psi_{\Theta}\right\|_{2}=1 .
$$

Let $x_{0} \in G$ satisfy (A.3) with $R:=\operatorname{dist}\left(x_{0}, \Theta\right.$ ), and set $A:=4 C_{1}>4$. For convenience we may assume $x_{0}=0$, in which case $\Theta \subset B(0, A R)$, and take $G=$ $B\left(0, A R+2 D_{0}\right)$.

Let us consider a function $\eta \in C_{\mathrm{c}}^{\infty}\left(\mathbb{R}^{d}\right)$ given by $\eta(x)=\xi(|x|)$, where $\xi$ is an even $C^{\infty}$ function on $\mathbb{R}$ such that

$$
\begin{aligned}
& 0 \leq \xi(s) \leq 1 \quad \text { for all } s \in \mathbb{R}, \\
& \xi(s)=0 \quad \text { if either }|s| \leq \delta / 8 \text { or }|s| \geq A R+D_{0}, \\
& \xi(s)=1 \quad \text { if } \delta / 4 \leq|s| \leq A R, \\
& \left|\xi^{(j)}(s)\right| \leq C_{4} \quad \text { for all } s \in \mathbb{R}, j=1,2,
\end{aligned}
$$

where $C_{4}=C_{4}\left(d, \delta, D_{0}\right)$ is a finite constant (independent of $A$ and $R$ ). Note that $|\nabla \eta| \leq$ $C_{4} \sqrt{d}$ and $|\Delta \eta| \leq C_{4} d$.

We now apply (A.12) to the function $\eta \psi$ with $\varrho=2 A R$. Given $\alpha \geq C_{2}>1$ (without loss of generality we take $C_{2}>1$ ), we get

$$
\begin{aligned}
\frac{\alpha^{3}}{3 C_{3} \varrho^{4}} \int_{\mathbb{R}^{d}} w_{\varrho}^{-1-2 \alpha} \eta^{2} \psi^{2} \mathrm{~d} x & \leq \frac{1}{3} \int_{\mathbb{R}^{d}} w_{\varrho}^{2-2 \alpha}(\Delta(\eta \psi))^{2} \mathrm{~d} x \\
\leq & \int_{\mathbb{R}^{d}} w_{\varrho}^{2-2 \alpha} \eta^{2}(\Delta \psi)^{2} \mathrm{~d} x+4 \int_{\operatorname{supp} \nabla \eta} w_{\varrho}^{2-2 \alpha}|\nabla \eta|^{2}|\nabla \psi|^{2} \mathrm{~d} x \\
& +\int_{\operatorname{supp} \nabla \eta} w_{\varrho}^{2-2 \alpha}(\Delta \eta)^{2} \psi^{2} \mathrm{~d} x
\end{aligned}
$$

where supp $\nabla \eta \subset\{\delta / 8 \leq|x| \leq \delta / 4\} \cup\left\{A R \leq|x| \leq A R+D_{0}\right\}$. 
It follows from (A.2), recalling $\|V\|_{\infty} \leq K$, and using also the fact that $w_{\varrho} \leq 1$ on supp $\eta$, that

$$
\begin{aligned}
\int_{\mathbb{R}^{d}} w_{\varrho}^{2-2 \alpha} \eta^{2}(\Delta \psi)^{2} \mathrm{~d} x & =\int_{\mathbb{R}^{d}} w_{\varrho}^{2-2 \alpha} \eta^{2}(V \psi-\zeta)^{2} \mathrm{~d} x \\
& \leq 2 K^{2} \int_{\mathbb{R}^{d}} w_{\varrho}^{-1-2 \alpha} \eta^{2} \psi^{2} \mathrm{~d} x+2 \int_{\mathbb{R}^{d}} w_{\varrho}^{2-2 \alpha} \eta^{2} \zeta^{2} \mathrm{~d} x
\end{aligned}
$$

We take

$$
\alpha=\alpha_{0} \rho^{4 / 3} \quad \text { where } \quad \alpha_{0} \geq \max \left\{\left(18 C_{3} K^{2}\right)^{1 / 3}, C_{2}\left(8 C_{1} D_{0}\right)^{-4 / 3}\right\},
$$

so we have

$$
\frac{\alpha^{3}}{3 C_{3} \varrho^{4}}=\frac{\alpha_{0}^{3}}{3 C_{3}} \geq 6 K^{2}
$$

Using (A.11) and (A.13), and recalling that diam $\Theta \leq D \leq R$, we have

$$
\int_{\mathbb{R}^{d}} w_{\varrho}^{-1-2 \alpha} \eta^{2} \psi^{2} \mathrm{~d} x \geq\left(\frac{\varrho}{R+\operatorname{diam} \Theta}\right)^{1+2 \alpha}\left\|\psi_{\Theta}\right\|_{2}^{2} \geq A^{1+2 \alpha}
$$

Combining (A.18), (A.19), (A.21), and (A.22), we conclude that

$$
\begin{aligned}
\frac{2 \alpha_{0}^{3}}{9 C_{3}} A^{1+2 \alpha} \leq & 4 \int_{\operatorname{supp} \nabla \eta} w_{\varrho}^{2-2 \alpha}|\nabla \eta|^{2}|\nabla \psi|^{2} \mathrm{~d} x \\
& +\int_{\operatorname{supp} \nabla \eta} w_{\varrho}^{2-2 \alpha}(\Delta \eta)^{2} \psi^{2} \mathrm{~d} x+2 \int_{\operatorname{supp} \eta} w_{\varrho}^{2-2 \alpha} \eta^{2} \zeta^{2} \mathrm{~d} x .
\end{aligned}
$$

We have

$$
\begin{aligned}
\int_{\left\{A R \leq|x| \leq A R+D_{0}\right\}} w_{\varrho}^{2-2 \alpha}\left(4|\nabla \eta|^{2}|\nabla \psi|^{2}+(\Delta \eta)^{2} \psi^{2}\right) \mathrm{d} x \\
\leq C_{4}^{2} d^{2}\left(\frac{C_{1} \varrho}{A R}\right)^{2 \alpha-2} \int_{\left\{A R \leq|x| \leq A R+D_{0}\right\}}\left(4|\nabla \psi|^{2}+\psi^{2}\right) \mathrm{d} x \\
\leq C_{5}\left(\frac{C_{1} \varrho}{A R}\right)^{2 \alpha-2} \int_{\left\{A R-D_{0} \leq|x| \leq A R+2 D_{0}\right\}}\left(\zeta^{2}+(1+K) \psi^{2}\right) \mathrm{d} x \\
\leq C_{5}\left(\frac{C_{1} \varrho}{A R}\right)^{2 \alpha-2}\left(\left\|\zeta_{G}\right\|_{2}^{2}+(1+K)\left\|\psi_{G}\right\|_{2}^{2}\right) \\
=C_{5}\left(2 C_{1}\right)^{2 \alpha-2}\left(\left\|\zeta_{G}\right\|_{2}^{2}+(1+K)\left\|\psi_{G}\right\|_{2}^{2}\right)
\end{aligned}
$$

where we used an interior estimate (e.g., [GK5, Lemma A.2]) and $C_{5}=C_{5}\left(d, \delta, D_{0}\right)$ is a constant. 
Similarly,

$$
\begin{aligned}
\int_{\{\delta / 8 \leq|x| \leq \delta / 4\}} w_{\varrho}^{2-2 \alpha}\left(4|\nabla \eta|^{2}|\nabla \psi|^{2}+(\Delta \eta)^{2} \psi^{2}\right) \mathrm{d} x \\
\leq C_{4}^{2} d^{2}\left(8 \delta^{-1} C_{1} \varrho\right)^{2 \alpha-2} \int_{\{\delta / 8 \leq|x| \leq \delta / 4\}}\left(4|\nabla \psi|^{2}+\psi^{2}\right) \mathrm{d} x \\
\leq C_{6}\left(8 \delta^{-1} C_{1} \varrho\right)^{2 \alpha-2} \int_{\{|x| \leq \delta / 2\}}\left(\zeta^{2}+(1+K) \psi^{2}\right) \mathrm{d} x \\
\leq C_{6}\left(8 \delta^{-1} C_{1} \varrho\right)^{2 \alpha-2}\left(\left\|\zeta_{G}\right\|_{2}^{2}+(1+K)\left\|\psi_{0, \delta}\right\|_{2}^{2}\right) \\
=C_{6}\left(16 \delta^{-1} C_{1} A R\right)^{2 \alpha-2}\left(\left\|\zeta_{G}\right\|_{2}^{2}+(1+K)\left\|\psi_{0, \delta}\right\|_{2}^{2}\right) \\
=C_{6}\left(64 \delta^{-1} C_{1}^{2} R\right)^{2 \alpha-2}\left(\left\|\zeta_{G}\right\|_{2}^{2}+(1+K)\left\|\psi_{0, \delta}\right\|_{2}^{2}\right),
\end{aligned}
$$

where $C_{6}=C_{6}\left(d, \delta, D_{0}\right)$ is a constant.

In addition,

$$
\begin{aligned}
2 \int_{\operatorname{supp} \eta} w_{\varrho}^{2-2 \alpha} \eta^{2} \zeta^{2} \mathrm{~d} x & \leq 2\left(8 \delta^{-1} C_{1} \varrho\right)^{2 \alpha-2}\left\|\zeta_{G}\right\|_{2}^{2} \\
& =2\left(64 \delta^{-1} C_{1}^{2} R\right)^{2 \alpha-2}\left\|\zeta_{G}\right\|_{2}^{2}
\end{aligned}
$$

Thus, if

$$
C_{5}(1+K)\left\|\psi_{G}\right\|_{2}^{2}\left(2 C_{1}\right)^{2 \alpha-2} \leq \frac{1}{2} \frac{2 \alpha_{0}^{3}}{9 C_{3}} A^{1+2 \alpha}=\frac{\alpha_{0}^{3}}{9 C_{3}}\left(4 C_{1}\right)^{1+2 \alpha},
$$

or, equivalently,

$$
\alpha_{0}^{3} 4^{\alpha} \geq \frac{9}{16} C_{5} C_{1}^{-2}(1+K)\left\|\psi_{G}\right\|_{2}^{2},
$$

we conclude that

$$
\begin{aligned}
\frac{\alpha_{0}^{3}}{9 C_{3}}\left(4 C_{1}\right)^{1+2 \alpha} \leq & C_{6}\left(64 \delta^{-1} C_{1}^{2} R\right)^{2 \alpha-2}(1+K)\left\|\psi_{0, \delta}\right\|_{2}^{2} \\
& +\left(\left(C_{6}+2\right)\left(64 \delta^{-1} C_{1}^{2} R\right)^{2 \alpha-2}+C_{5}\left(2 C_{1}\right)^{2 \alpha-2}\right)\left\|\zeta_{G}\right\|_{2}^{2} \\
\leq & C_{7}\left(\beta_{1} \delta^{-1} C_{1}^{2} R\right)^{2 \alpha-2}\left((1+K)\left\|\psi_{0, \delta}\right\|_{2}^{2}+\left\|\zeta_{G}\right\|_{2}^{2}\right),
\end{aligned}
$$

where we used $R \geq D \geq D_{0}$, set $C_{7}=\max \left\{C_{5}, C_{6}+2\right\}$, and took

$$
\beta_{1}=\max \left\{64,2 \delta\left(C_{1} D_{0}\right)^{-1}\right\} .
$$

It follows that

$$
C_{8} \frac{\alpha_{0}^{3}}{C_{3}}(\beta R)^{-2 \alpha} \leq(1+K)\left\|\psi_{0, \delta}\right\|_{2}^{2}+\left\|\zeta_{G}\right\|_{2}^{2},
$$

with a constant $C_{8}=C_{8}\left(d, \delta, D_{0}, C_{1}\right)>0$ and

$$
\beta=\frac{1}{4} \beta_{1} \delta^{-1} C_{1}=\max \left\{16 \delta^{-1} C_{1},\left(2 D_{0}\right)^{-1}\right\} .
$$


Since $R \geq D$ and we require (A.20), to satisfy (A.28) it suffices to also require

$$
4^{\alpha_{0}\left(4 C_{1} D_{0}\right)^{4 / 3}} \geq \frac{9}{16} C_{2}^{-3}\left(8 C_{1} D_{0}\right)^{4} C_{5} C_{1}^{-2}(1+K)\left\|\psi_{G}\right\|_{2}^{2},
$$

that is,

$$
\alpha_{0} \geq\left(4 C_{1} D_{0}\right)^{-4 / 3}(\log 4)^{-1} \log \left(C_{9}(1+K)\left\|\psi_{G}\right\|_{2}^{2}\right),
$$

where $C_{9}=C_{9}\left(d, \delta, D_{0}\right)$.

Thus we can satisfy (A.20) and (A.28) by taking

$$
\alpha=\alpha_{1} R^{4 / 3} \quad \text { with } \quad \alpha_{1}=C_{10}\left(1+K^{2 / 3}+\log \left\|\psi_{G}\right\|_{2}\right),
$$

for some appropriate constant $C_{10}=C_{10}\left(d, \delta, D_{0}\right)$.

It now follows from (A.31), (A.32) and (A.35) that we can find a constant $m=$ $m\left(d, \delta, D_{0}\right)>0$ such that

$$
R^{-m\left(1+K^{2 / 3}+\log \left\|\psi_{G}\right\|_{2}\right) R^{4 / 3}} \leq(1+K)\left\|\psi_{0, \delta}\right\|_{2}^{2}+\left\|\zeta_{G}\right\|_{2}^{2} \quad \text { for all } R \geq D .
$$

Proof of Corollary A.2. Without loss of generality we take $x_{0}=0$, i.e., $\Lambda=\Lambda_{L}(0)$. We will prove the corollary for the case of a Dirichlet boundary condition, the modifications for the (easier) case of a periodic boundary condition will be obvious.

Let $\Delta_{\Lambda}$ be the Dirichlet Laplacian on $\Lambda$, and let $V$ be a bounded potential on $\Lambda$ with $\|V\|_{\infty} \leq K<\infty$. Given $\varphi \in \mathrm{L}^{2}(\Lambda)$, we extend it to a function $\widetilde{\varphi} \in \mathrm{L}_{\text {loc }}^{2}\left(\mathbb{R}^{d}\right)$ by setting $\widetilde{\varphi}=\varphi$ on $\Lambda$ and $\tilde{\varphi}=0$ on $\partial \Lambda$, and requiring that for all $x \in \mathbb{R}^{d}$ and $j \in\{1, \ldots, d\}$ we have

$$
\widetilde{\varphi}(x)=-\widetilde{\varphi}\left(x+\left(L-2 \widehat{x_{j}}\right) \mathrm{e}_{j}\right),
$$

where $\left\{\mathrm{e}_{j}\right\}_{j=1, \ldots, d}$ is the canonical orthonormal basis in $\mathbb{R}^{d}$, and for each $t \in \mathbb{R}$ we define $\hat{t} \in]-L / 2, L / 2]$ by $t=k L+\hat{t}$ with $k \in \mathbb{Z}$. Note that if $\left.\Lambda^{\prime}=\Lambda_{L^{\prime}}(0)=\right]-L^{\prime} / 2, L^{\prime} / 2\left[^{d}\right.$, we have

$$
\left\|\widetilde{\varphi}_{\Lambda^{\prime}}\right\|_{2}^{2}=(2 n+1)^{d}\left\|\varphi_{\Lambda}\right\|_{2}^{2} \quad \text { if } L^{\prime}=(2 n+1) L \text { for some } n \in \mathbb{N} .
$$

We also extend the potential $V$ to a potential $\widehat{V}$ on $\mathbb{R}^{d}$ by by setting $\widehat{V}=V$ on $\Lambda$ and $V=0$ on $\partial \Lambda$, and requiring that for all $x \in \mathbb{R}^{d}$ and $j \in\{1, \ldots, d\}$ we have

$$
\widehat{V}(x)=\widehat{V}\left(x+\left(L-2 \widehat{x}_{j}\right) \mathrm{e}_{j}\right) .
$$

In particular, $\|\widehat{V}\|_{\infty}=\|V\|_{\infty} \leq K$.

Using the fact that for all eigenfunctions $\phi$ of $\Delta_{\Lambda}$ (given explicitly in [RS, Eq. (113) in Chapter XIII]) we have $\widetilde{\phi} \in C^{\infty}\left(\mathbb{R}^{d}\right)$, we conclude that $\psi \in \mathcal{D}\left(\Delta_{\Lambda}\right)$ implies $\widetilde{\psi} \in$ $\mathrm{H}_{\text {loc }}^{2}\left(\mathbb{R}^{d}\right)$, satisfying

$$
-\Delta \widetilde{\psi}+\widehat{V} \widetilde{\psi}=\widetilde{H_{\Lambda} \psi} \quad \text { a.e. in } \mathbb{R}^{d} .
$$

Now let $\delta, D, \Theta$ be as in Corollary A.2(i), and set $D_{0}=D$. In view of (A.5) we may assume $D \leq R \leq \sqrt{d} L$ without loss of generality. We take $\Lambda_{1}=\Lambda_{L_{1}}(0)$, with

$$
L_{1}=\left(2\left[\left[\left(4 C_{1}+2\right) \sqrt{d}\right]\right]+1\right) L \leq 29 \sqrt{d} L,
$$


where $[[t]]$ denotes the smallest integer greater than or equal to $t$, and we used (A.1). Fix $x \in \Lambda$ satisfying (A.5), it follows that $x$ satisfies (A.3) with $G=\Lambda_{1}$. We now apply Theorem A.1 with $G=\Lambda_{1}$. Given $\psi \in \mathcal{D}\left(\Delta_{\Lambda}\right), \widetilde{\psi}$ satisfies (A.40) on $\Lambda_{1}$, and hence (A.4) yields

$$
(1+K)\left\|\widetilde{\psi}_{x, \delta}\right\|_{2}^{2}+\left\|\left(\widetilde{H_{\Lambda} \psi}\right)_{\Lambda_{1}}\right\|_{2}^{2} \geq R^{-m\left(1+K^{2 / 3}+\log \left(\left\|\widetilde{\psi}_{\Lambda_{1}}\right\|_{2}\left\|\widetilde{\psi}_{\Theta}\right\|_{2}^{-1}\right)\right) R^{4 / 3}}\left\|\widetilde{\psi}_{\Theta}\right\|_{2}^{2},
$$

with a constant $m=m(d, \delta, D)>0$. Taking into account (A.37), (A.38), and (A.41), we get (A.6).

To prove Corollary A.2(ii), let $L \geq 2,0<\delta \leq L$, and $x \in \Lambda$ with $B(x, \delta / 2) \subset \Lambda$. We take $\Lambda_{2}=\Lambda_{L_{2}}(0)$ with

$$
L_{2}=\left(2\left[\left[\left(6 C_{1}+3\right)\right]\right]+1\right) L \leq 41 L,
$$

where we used (A.1). We let $\Theta_{x}=\Lambda+2 L \mathrm{e}^{(x)} \subset \Lambda_{2}$, where $\mathrm{e}^{(x)} \in\left\{ \pm \mathrm{e}_{j}\right\}_{j=1, \ldots, d}$ is chosen such that $R:=\operatorname{dist}\left(x, \Theta_{x}\right) \in[L, 3 / 2 L]$. It follows that $x$ satisfies (A.3) with $G=\Lambda_{2}$, so we apply Theorem A.1 with $G=\Lambda_{2}, D_{0}=\delta / 2, D=L$, and $\Theta=\Theta_{x}$. Given $\psi \in \mathcal{D}\left(\Delta_{\Lambda}\right), \widetilde{\psi}$ satisfies (A.40) on $\Lambda_{2}$, we have $\left\|\widetilde{\psi}_{\Theta}\right\|_{2}=\left\|\psi_{\Lambda}\right\|_{2}$, and hence (A.4) yields

$$
(1+K)\left\|\widetilde{\psi}_{x, \delta}\right\|_{2}^{2}+\left\|\left(\widetilde{H_{\Lambda} \psi}\right)_{\Lambda_{2}}\right\|_{2}^{2} \geq(3 L / 2)^{-m^{\prime}\left(1+K^{2 / 3}\right)(3 L / 2)^{4 / 3}}\left\|\psi_{\Lambda}\right\|_{2},
$$

with a constant $m^{\prime}=m^{\prime}(d, \delta)>0$. Using (A.37), (A.38), and (A.43), we get (A.7)

\section{A.2. Application to Schrödinger operators with periodic potentials}

Consider the Schrödinger operator $H=-\Delta+V$ on $\mathrm{L}^{2}\left(\mathbb{R}^{d}\right)$, where $\Delta$ is the $d$-dimensional Laplacian operator and $V$ is a bounded periodic potential with period $q>0$, i.e., periodic with respect to the group $q \mathbb{Z}^{d}$. Without loss of generality we assume inf $\sigma(H)=0$, i.e., $0 \in \sigma(H) \subset[0, \infty[$.

Given $\delta \in] 0, q]$, we set $b_{\delta}=\chi_{B(0, \delta / 2)}$, and consider the $q$-periodic bounded operator $W_{\delta}$ on $\mathrm{L}^{2}\left(\mathbb{R}^{d}\right)$ given by multiplication by the function

$$
W_{\delta}(x)=\sum_{m \in q \mathbb{Z}^{d}} b_{\delta}(x-m) .
$$

We also consider the corresponding finite volume operators. Given $L \in q \mathbb{N}$, we set $H_{L}=-\Delta_{L}+V$ on $\mathrm{L}^{2}\left(\Lambda_{L}, \mathrm{~d} x\right)$, where $\Lambda_{L}=\Lambda_{L}(0) . \Delta_{L}$ is the Laplacian with a periodic boundary condition on $\Lambda_{L}$, which we identify with the torus $\mathbb{R}^{d} / L \mathbb{Z}^{d}$ in the usual way. We will also write $H_{\infty}=H$.

Combes, Hislop and Klopp [CoHK1, Section 4] proved that for every compact interval $I$ there exists a constant $C_{I, \delta}=C_{d, V, I, \delta}>0$, such that for all $L \in q \mathbb{N} \cup\{\infty\}$ we have

$$
\chi_{I}\left(H_{L}\right) W_{\delta} \chi_{I}\left(H_{L}\right) \geq C_{I, \delta} \chi_{I}\left(H_{L}\right) .
$$

Their proof relies on the unique continuation principle for Schrödinger operators, and for this reason does not provide much information on the constant $C_{I, \delta}>0$. We will show that the quantitative unique continuation principle can be used to prove a modified form of their result with control of the constant. 
Theorem A.6. Let $H=-\Delta+V$ be a periodic Schrödinger operator on $\mathrm{L}^{2}\left(\mathbb{R}^{d}\right)$ as above, with period $q \geq 2$, and let $W_{\delta}$ be as in (A.45). Given $E_{0}>0$, set $K_{0}=E_{0}+$ $\|V\|_{\infty}$. There exists a constant $\widehat{m}=\widehat{m}(d, \delta)>0$ such that, defining $\gamma>0$ by

$$
\gamma^{2}=\frac{1}{2}(41)^{-d} q^{-\widehat{m}\left(1+K_{0}^{2 / 3}\right) q^{4 / 3}},
$$

for any closed interval $I \subset\left[0, E_{0}\right]$ with $|I| \leq 2 \gamma$ and any scale $L \in q \mathbb{N} \cup\{\infty\}$ we have

$$
\chi_{I}\left(H_{L}\right) W_{\delta} \chi_{I}\left(H_{L}\right) \geq(41)^{d} \gamma^{2}\left(1+K_{0}\right)^{-1} \chi_{I}\left(H_{L}\right) .
$$

Proof. We will need to review Floquet Theory (see [RS, Section XIII.6]). We let $Q=$ $\Lambda_{q}(0)$ be the basic period cell, and $\widetilde{Q}=\Lambda_{2 \pi / q}(0)$ the dual basic cell. We define the Floquet transform

$$
\mathcal{F}: \mathrm{L}^{2}\left(\mathbb{R}^{d}, \mathrm{~d} x\right) \rightarrow \int_{\widetilde{Q}}^{\oplus} \mathrm{L}^{2}(Q, \mathrm{~d} x) \mathrm{d} k \cong L^{2}\left(\widetilde{Q}, \mathrm{~d} k ; \mathrm{L}^{2}(Q, \mathrm{~d} x)\right)
$$

by

$$
(\mathcal{F} \psi)(k, x)=\left(\frac{q}{2 \pi}\right)^{d / 2} \sum_{m \in q \mathbb{Z}^{d}} \mathrm{e}^{-i k \cdot m} \psi(x-m), \quad x \in Q, k \in \widetilde{Q},
$$

if $\psi$ has compact support; it extends by continuity to a unitary operator.

The $q$-periodic operator $H$ is decomposable in this direct integral representation, more precisely,

$$
\mathcal{F} H \mathcal{F}^{*}=\int_{\widetilde{Q}}^{\oplus} H_{Q}(k) \mathrm{d} k
$$

where for each $k \in \mathbb{R}^{d}$ we set $H_{Q}(k)=-\Delta_{Q}(k)+V$, where $\Delta_{Q}(k)$ is the Laplacian on $Q$ with a $k$-quasi-periodic boundary condition, i.e., defined on functions of the form $\psi(x)=\mathrm{e}^{-i k \cdot x} \varphi(x)$ with $\varphi$ a periodic function on $Q$. Note that $H_{Q}(0)=H_{q}$. Moreover, if $p \in(2 \pi / q) \mathbb{Z}^{d}$, then for all $k \in \mathbb{R}^{d}$ we have $H_{Q}(k+p)=\mathrm{e}^{-i p \cdot x} H_{Q}(k) \mathrm{e}^{i p \cdot x}$.

If $L \in q \mathbb{N}$, similar considerations apply to the operator $H_{L}$, which is $q$-periodic on the torus $\Lambda_{L} \cong \mathbb{R}^{d} / L \mathbb{Z}^{d}$. The Floquet transform

$$
\mathcal{F}_{L}: \mathrm{L}^{2}\left(\Lambda_{L}, \mathrm{~d} x\right) \rightarrow \bigoplus_{k \in \frac{2 \pi}{L} \mathbb{Z}^{d} \cap \widetilde{Q}} \mathrm{~L}^{2}(Q, \mathrm{~d} x)
$$

is a unitary operator now defined by

$$
\left(\mathcal{F}_{L} \psi\right)(k, x)=(q / L)^{d / 2} \sum_{m \in q \mathbb{Z}^{d} \cap \Lambda_{L}} \mathrm{e}^{-i k \cdot m} \psi(x-m),
$$

where $x \in Q, k \in \frac{2 \pi}{L} \mathbb{Z}^{d} \cap \widetilde{Q}, \psi \in \mathrm{L}^{2}\left(\Lambda_{L}, \mathrm{~d} x\right)$, and $\psi(x-m)$ is properly interpreted in the torus $\Lambda_{L}$. We also have

$$
\mathcal{F}_{L} H_{L} \mathcal{F}_{L}^{*}=\bigoplus_{k \in \frac{2 \pi}{L} \mathbb{Z}^{d} \cap \widetilde{Q}} H_{Q}(k)
$$


It follows that for any bounded Borel function $f$ we have

$$
\mathcal{F} f(H) \mathcal{F}^{*}=\int_{\widetilde{Q}}^{\oplus} f\left(H_{Q}(k)\right) \mathrm{d} k, \quad \mathcal{F}_{L} f\left(H_{L}\right) \mathcal{F}_{L}^{*}=\bigoplus_{k \in \frac{2 \pi}{L} \mathbb{Z}^{d} \cap \widetilde{Q}} f\left(H_{Q}(k)\right) .
$$

Let us fix $\delta \in] 0, q]$ and $E_{0}>0$. We set $K_{0}=\|V\|_{\infty}+E_{0}$, so $\|V-E\|_{\infty} \leq K_{0}$ for all $E \in I_{0}$. Given $k \in \widetilde{Q}$, we consider the Schrödinger operator $H_{Q}(k)$ on $\mathrm{L}^{\overline{2}}(Q)$, and proceed similarly to the proof of Corollary A.2(ii). Since we have a $k$-quasi-periodic boundary condition, we extend a function $\varphi \in \mathrm{L}^{2}(Q)$ to a function $\widetilde{\varphi} \in \mathrm{L}_{\text {loc }}^{2}\left(\mathbb{R}^{d}\right)$ by requiring $\widetilde{\varphi}=\varphi$ on $Q$ and $\widetilde{\varphi}(x+m)=\mathrm{e}^{-i k \cdot m} \widetilde{\varphi}(x)$ for all $x \in \mathbb{R}^{d}$ and $m \in q \mathbb{Z}^{d}$. If $\psi \in \mathcal{D}\left(\Delta_{Q}(k)\right)$, then $\widetilde{\psi} \in \mathrm{H}_{\text {loc }}^{2}\left(\mathbb{R}^{d}\right)$ and we have

$$
-\Delta \widetilde{\psi}+V \widetilde{\psi}=\widetilde{H_{Q}(k) \psi} \quad \text { a.e. in } \mathbb{R}^{d} .
$$

We apply Theorem A.1 with $G=\Lambda_{L_{2}}(0)$, where $L_{2}$ is given in (A.43) (recall $L=q$ ). Proceeding as in the derivation of (A.44) and (A.7), using $q \geq 2$, we get

$$
\left(1+K_{0}\right)\left\|\left(b_{\delta} \psi\right)_{Q}\right\|_{2}^{2}+(41)^{d}\left\|\left(\left(H_{Q}(k)-E\right) \psi\right)_{Q}\right\|_{2}^{2} \geq q^{-\widehat{m}\left(1+K_{0}^{2 / 3}\right) q^{4 / 3}}\left\|\psi_{Q}\right\|_{2}^{2}
$$

for all $E \in\left[0, E_{0}\right]$, with a constant $\widehat{m}=\widehat{m}(d, \delta)>0$.

We now take $I=[E-\varepsilon, E+\varepsilon] \subset\left[0, E_{0}\right]$. If $\psi=\chi_{I}\left(H_{Q}(k)\right) \psi$, we have

$$
\left\|\left(\left(H_{Q}(k)-E\right) \psi\right)_{Q}\right\|_{2} \leq \varepsilon\left\|\psi_{Q}\right\|_{2} \text {, }
$$

and it follows from (A.57) that

$$
\left(1+K_{0}\right)\left\|\left(b_{\delta} \psi\right)_{Q}\right\|_{2}^{2}+\varepsilon^{2}(41)^{d}\left\|\psi_{Q}\right\|_{2}^{2} \geq q^{-\widehat{m}\left(1+K_{0}^{2 / 3}\right) q^{4 / 3}}\left\|\psi_{Q}\right\|_{2}^{2} .
$$

Thus, if $\varepsilon \leq \gamma$, where $\gamma$ is given in (A.47), we get

$$
\left(1+K_{0}\right)\left\|\left(b_{\delta} \psi\right)_{Q}\right\|_{2}^{2} \geq \frac{1}{2} q^{-\widehat{m}\left(1+K_{0}^{2 / 3}\right) q^{4 / 3}}\left\|\psi_{Q}\right\|_{2}^{2}=(41)^{d} \gamma^{2}\left\|\psi_{Q}\right\|_{2}^{2},
$$

that is,

$$
\chi_{I}\left(H_{Q}(k)\right) b_{\delta} \chi_{I}\left(H_{Q}(k)\right) \geq(41)^{d} \gamma^{2}\left(1+K_{0}\right)^{-1} \chi_{I}\left(H_{Q}(k)\right) .
$$

Given an interval $I$, we have

$$
\mathcal{F}\left\{\chi_{I}(H) W_{\delta} \chi_{I}(H)\right\} \mathcal{F}^{*}=\int_{\widetilde{Q}}^{\oplus}\left\{\chi_{I}\left(H_{Q}(k)\right) b_{\delta} \chi_{I}\left(H_{Q}(k)\right)\right\} \mathrm{d} k,
$$

and, for $L \in q \mathbb{N}$,

$$
\mathcal{F}_{L}\left\{\chi_{I}\left(H_{L}\right) W_{\delta} \chi_{I}\left(H_{L}\right)\right\} \mathcal{F}_{L}^{*}=\bigoplus_{k \in \frac{2 \pi}{L} \mathbb{Z}^{d} \cap \tilde{Q}}\left\{\chi_{I}\left(H_{Q}(k)\right) b_{\delta} \chi_{I}\left(H_{Q}(k)\right)\right\} .
$$

Thus for $I=[E-\varepsilon, E+\varepsilon] \subset\left[0, E_{0}\right]$, with $\varepsilon \leq \gamma$, it follows from (A.61)-(A.63) that for all $L \in q \mathbb{N} \cup\{\infty\}$ we have

$$
\chi_{I}\left(H_{L}\right) W_{\delta} \chi_{I}\left(H_{L}\right) \geq(41)^{d} \gamma^{2}\left(1+K_{0}\right)^{-1} \chi_{I}\left(H_{L}\right),
$$

so we proved (A.48). 
Remark A.7. Note that (A.48) holds for $I=\left[0, E_{1}\right]$ where

$$
E_{1}^{2}=2(41)^{-d} q^{-\widehat{m}\left(1+\left(\|V\|_{\infty}+E_{1}\right)^{2 / 3}\right) q^{4 / 3}} .
$$

Note that this equation has a solution $E_{1}>0$.

Acknowledgments. François Germinet was partially supported by the ANR BLAN 0261. Abel Klein was supported in part by the NSF under grant DMS-1001509.

\section{References}

[A] Aizenman, M.: Localization at weak disorder: some elementary bounds. Rev. Math. Phys. 6, 1163-1182 (1994) Zbl 0843.47039 MR 1301371

[ASFH] Aizenman, M., Schenker, J., Friedrich, R., Hundertmark, D.: Finite volume fractionalmoment criteria for Anderson localization. Comm. Math. Phys. 224, 219-253 (2001) Zbl 1038.82038 MR 1868998

[AENSS] Aizenman, M., Elgart, A., Naboko, S., Schenker, J., Stolz, G.: Moment analysis for localization in random Schrödinger operators. Invent. Math. 163, 343-413 (2006) Zbl 1090.81026 MR 2207021

[AGKW] Aizenman, M., Germinet, F., Klein, A., Warzel, S.: On Bernoulli decompositions for random variables, concentration bounds, and spectral localization. Probab. Theory Related Fields 143, 219-238 (2009) Zbl 1152.60020 MR 2449128

[AM] Aizenman, M., Molchanov, S.: Localization at large disorder and extreme energies: an elementary derivation. Comm. Math. Phys. 157, 245-278 (1993) Zbl 0782.60044 MR 1244867

[BGK] Bouclet, J. M., Germinet, F., Klein, A.: Sub-exponential decay of operator kernels for functions of generalized Schrödinger operators. Proc. Amer. Math. Soc. 132, 27032712 (2004) Zbl 1053.81028 MR 2054797

[Bo] Bourgain, J.: On localization for lattice Schrödinger operators involving Bernoulli variables. In: Geometric Aspects of Functional Analysis, Lecture Notes in Math. 1850, Springer, Berlin, 77-99 (2004) Zbl 1083.35073 MR 2087153

[BoK] Bourgain, J., Kenig, C.: On localization in the continuous Anderson-Bernoulli model in higher dimension. Invent. Math. 161, 389-426 (2005) Zbl 1084.82005 MR 2180453

[CKM] Carmona, R., Klein, A., Martinelli, F.: Anderson localization for Bernoulli and other singular potentials. Comm. Math. Phys. 108, 41-66 (1987) Zbl 0615.60098 MR 0872140

[CL] Carmona, R, Lacroix, J.: Spectral Theory of Random Schrödinger Operators. Birkhäuser, Boston (1990) Zbl 0717.60074 MR 1102675

[CoH1] Combes, J. M., Hislop, P. D.: Localization for some continuous, random Hamiltonians in $d$-dimension. J. Funct. Anal. 124, 149-180 (1994) Zbl 0801.60054 MR 1284608

[CoH2] Combes, J. M., Hislop, P. D.: Landau Hamiltonians with random potentials: localization and the density of states. Comm. Math. Phys. 177, 603-629 (1996) Zbl 0851.60099 MR 1385078

[CoHK1] Combes, J. M., Hislop, P. D., Klopp, F.: Hölder continuity of the integrated density of states for some random operators at all energies. Int. Math. Res. Notices 4, 179-209 (2003) Zbl 1022.47028 MR 1935272 
[CoHK2] Combes, J. M., Hislop, P. D., Klopp, F.: An optimal Wegner estimate and its application to the global continuity of the integrated density of states for random Schrödinger operators. Duke Math. J. 140, 469-498 (2007) Zbl 1134.81022 MR 2362242

[CoHKN] Combes, J. M., Hislop, P. D., Klopp, F., Nakamura, S.: The Wegner estimate and the integrated density of states for some random operators. In: Spectral and Inverse Spectral Theory (Goa, 2000), Proc. Indian Acad. Sci. Math. Sci. 112 , 31-53 (2002) Zbl 1009.47060 MR 1894541

[CoHM] Combes, J. M., Hislop, P. D., Mourre, E.: Spectral averaging, perturbation of singular spectra, and localization. Trans. Amer. Math. Soc. 348, 4883-4894 (1996) Zbl 0868.35081 MR 1344205

[CoHN] Combes, J. M., Hislop, P. D., Nakamura, S.: The $\mathrm{L}^{p}$-theory of the spectral shift function, the Wegner estimate and the integrated density of states for some random operators. Comm. Math. Phys. 218, 113-130 (2001) Zbl 1042.82024 MR 1824200

[CrS] Craig, W., Simon, B.: Log Hölder continuity of the integrated density of states for stochastic Jacobi matrices. Comm. Math. Phys. 90, 207-218 (1983) Zbl 0532.60057 MR 0714434

[DSS] Damanik, D., Sims, R., Stolz, G.: Localization for one dimensional, continuum, Bernoulli-Anderson models. Duke Math. J. 114, 59-100 (2002) Zbl 1107.82025 MR 1915036

[DS] Damanik, D., Stollmann, P.: Multi-scale analysis implies strong dynamical localization. Geom. Funct. Anal. 11, 11-29 (2001) Zbl 0976.60064 MR 1829640

[Da] Davey, B.: PhD thesis. In preparation (private communication by C. Kenig)

[Dav] Davies, E. B.: Spectral Theory and Differential Operators. Cambridge Univ. Press (1995) MR 1349825

[DeRJLS] Del Rio, R., Jitomirskaya, S., Last, Y., Simon, B.: Operators with singular continuous spectrum IV: Hausdorff dimensions, rank one pertubations and localization. J. Anal. Math. 69, 153-200 (1996) Zbl 0908.47002 MR 1428099

[DelLS] Delyon, F., Lévy, Y., Souillard, B.: Anderson localization for multidimensional systems at large disorder or large energy. Comm. Math. Phys. 100, 463-470 (1985) Zbl 0576.60053 MR 0806247

[DelS] Delyon, F., Lévy, Y., Souillard, B.: Remark on the continuity of the density of states of ergodic finite difference operators. Comm. Math. Phys. 94, 289-291 (1984) Zbl 0546.60065 MR 0761798

[DoIM] Doi, S., Iwatsuka, A., Mine, T.: The uniqueness of the integrated density of states for the Schrödinger operators with magnetic fields. Math. Z. 237, 335-371 (2001) Zbl 0985.35055 MR 1838315

[Dr] von Dreifus, H.: On the effects of randomness in ferromagnetic models and Schrödinger operators. Ph. D. thesis, New York Univ. (1987) MR 2636321

[DrK1] von Dreifus, H., Klein, A.: A new proof of localization in the Anderson tight binding model. Comm. Math. Phys. 124, 285-299 (1989) Zbl 0698.60051 MR 1012868

[DrK2] von Dreifus, H., Klein, A.: Localization for random Schrodinger operators with correlated potentials. Comm. Math. Phys. 140, 133-147 (1991) Zbl 0734.60070 MR 1124263

[FK1] Figotin, A., Klein, A.: Localization phenomenon in gaps of the spectrum of random lattice operators. J. Statist. Phys. 75, 997-1021 (1994) Zbl 0838.47050 MR 1285295

[FK2] Figotin, A., Klein, A.: Localization of classical waves I: Acoustic waves. Comm. Math. Phys. 180, 439-482 (1996) Zbl 0878.35109 MR 1405959 
[FK3] Figotin, A., Klein, A.: Midgap defect modes in dielectric and acoustic media. SIAM J. Appl. Math. 58, 1748-1773 (1998) Zbl 0963.78005 MR 1638668

[FiLM] Fischer, W., Leschke, H., Müller, P.: Spectral localization by Gaussian random potentials in multi-dimensional continuous space. J. Statist. Phys. 101, 935-985 (2000) Zbl 1049.82034 MR 1806712

[FrMSS] Fröhlich, J.: Martinelli, F., Scoppola, E., Spencer, T.: Constructive proof of localization in the Anderson tight binding model. Comm. Math. Phys. 101, 21-46 (1985) Zbl 0573.60096 MR 0814541

[FrS] Fröhlich, J., Spencer, T.: Absence of diffusion with Anderson tight binding model for large disorder or low energy. Comm. Math. Phys. 88, 151-184 (1983) Zbl 0519.60066 MR 0696803

[GDB] Germinet, F., De Bièvre, S.: Dynamical localization for discrete and continuous random Schrödinger operators. Comm. Math. Phys. 194, 323-341 (1998) Zbl 0911.60099 MR 1627657

[GHK1] Germinet, F., Hislop, P., Klein, A.: On localization for the Schrödinger operator with a Poisson random potential. C. R. Math. Acad. Sci. Paris 341, 525-528 (2005) Zbl 1083.35078 MR 2180822

[GHK2] Germinet, F., Hislop, P., Klein, A.: Localization for Schrödinger operators with Poisson random potential. J. Eur. Math. Soc. 9, 577-607 (2007) Zbl 1214.82053 MR 2314108

[GHK3] Germinet, F., Hislop, P., Klein, A.: Localization at low energies for attractive Poisson random Schrödinger operators. In: Probability and Mathematical Physics: A Volume in Honor of Stanislav Molchanov, CRM Proc. Lecture Notes 42, Amer. Math. Soc., 153-165 (2007) Zbl 1149.35064 MR 2352267

[GK1] Germinet, F., Klein, A.: Bootstrap multiscale analysis and localization in random media. Comm. Math. Phys. 222, 415-448 (2001) Zbl 0982.82030 MR 1859605

[GK2] Germinet, F., Klein, A.: Operator kernel estimates for functions of generalized Schrödinger operators. Proc. Amer. Math. Soc. 131, 911-920 (2003) Zbl 1013.81009 MR 1937430

[GK3] Germinet, F., Klein, A.: Explicit finite volume criteria for localization in continuous random media and applications. Geom. Funct. Anal. 13, 1201-1238 (2003) Zbl 1086.82008 MR 2033837

[GK4] Germinet, F., Klein, A.: High disorder localization for random Schrödinger operators through explicit finite volume criteria. Markov Process. Related Fields 9, 633-650 (2003) Zbl 1213.82045 MR 2072246

[GK5] Germinet, F., Klein, A.: A characterization of the Anderson metal-insulator transport transition. Duke Math. J. 124, 309-351 (2004) Zbl 1062.82020 MR 2078370

[GK6] Germinet, F., Klein, A.: New characterizations of the region of complete localization for random Schrödinger operators. J. Statist. Phys. 122, $73-94$ (2006) Zbl 1127.82031 MR 2203782

[GK7] Germinet, F., Klein, A.: Localization for some Cantor-Anderson Schrödinger operators. In: Adventures in Mathematical Physics, Contemp. Math. 447, Amer. Math. Soc., Providence, RI, 103-112 (2007) Zbl 1137.81011 MR 2423574

[GKM] Germinet, F., Klein, A., Mandy, B.: Dynamical delocalization in random Landau Hamiltonians with unbounded random couplings. In: Spectral and Scattering Theory for Quantum Magnetic Systems, Contemp. Math. 500, Amer. Math. Soc., Providence, RI, 87-100 (2009) Zbl 1193.82019 MR 2655144

[GKS1] Germinet, F., Klein, A., Schenker, J.: Dynamical delocalization in random Landau Hamiltonians. Ann. of Math. 166, 215-244 (2007) Zbl 1159.82009 MR 2342695 
[GKS2] Germinet, F., Klein, A., Schenker, J.: Quantization of the Hall conductance and delocalization in ergodic Landau Hamiltonians. Rev. Math. Phys. 21, 1045-1080 (2009) MR 25680511180.82099

[GhK] Ghribi, F., Klopp, F.: Localization for the random displacement model at weak disorder. Ann. Henri Poincaré 11, 127-149 (2010) Zbl 1210.82063 MR 2658987

[GoMP] Gol'dsheid, Ya., Molchanov, S., Pastur, L.: Pure point spectrum of stochastic one dimensional Schrödinger operators. Funct. Anal. Appl. 11, 1-10 (1977) Zbl 0368.34015 MR 0470515

[HK] Hislop, P. D., Klopp, F.: The integrated density of states for some random operators with nonsign definite potentials. J. Funct. Anal. 195, 12-47 (2002) Zbl 1013.60046 MR 1934351

[Ho] Hoeffding, W.: Probability inequalities for sums of bounded random variables. J. Amer. Statist. Assoc. 58, 13-30 (1963) Zbl 0127.10602 MR 0144363

[HolM] Holden, H., Martinelli, F.: On absence of diffusion near the bottom of the spectrum for a random Schrödinger operator. Comm. Math. Phys. 93, 197-217 (1984) Zbl 0546.60063 MR 0742193

[HuS] Hunziker, W., Sigal, I. M.: Time-dependent scattering theory of $N$-body quantum systems. Rev. Math. Phys. 12, 1033-1084 (2000) Zbl 0978.47008 MR 1791435

[J] Jitomirskaya, S.: Ergodic Schrödinger operators (on one foot). In: Spectral theory and Mathematical Physics: a Festschrift in honor of Barry Simon's 60th birthday. Proc. Sympos. Pure Math. 76, Part 2, Amer. Math. Soc., Providence, RI, 613-647 (2007) Zbl 1129.82018 MR 2307750

[K] Kato, T.: Perturbation Theory for Linear Operators. Springer (1976) Zbl 0342.47009 MR 0407617

[Ki] Kirsch, W.: Wegner estimates and Anderson localization for alloy-type potentials. Math. Z. 221, 507-512 (1996) Zbl 0863.35069 MR 1381595

[KiM1] Kirsch, W., Martinelli, F.: On the ergodic properties of the spectrum of general random operators. J. Reine Angew. Math. 334, 141-156 (1982) Zbl 0476.60058 MR 0667454

[KiM2] Kirsch, W., Martinelli, F. : On the spectrum of Schrödinger operators with a random potential. Comm. Math. Phys. 85, 329-350 (1982) Zbl 0506.60058 MR 0678150

[KiSS1] Kirsch, W., Stollman, P., Stolz, G.: Localization for random perturbations of periodic Schrödinger operators. Random Oper. Stochastic Equations 6, 241-268 (1998) Zbl 0927.60067 MR 1630995

[KiSS2] Kirsch, W., Stollmann, P, Stolz, G.: Anderson localization for random Schrödinger operators with long range interactions. Comm. Math. Phys. 195, 495-507 (1998) Zbl 0928.47049 MR 1640991

[K11] Klein, A.: Localization in the Anderson model with long range hopping. Brazilian J. Phys. 23, 363-371 (1993)

[K12] Klein, A.: Multiscale analysis and localization of random operators. In: Random Schrödinger Operators, Panoramas et Synthèses 25, Soc. Math. France, Paris, 121159 (2008) Zbl 1187.82058 MR 2509111

[KIKS] Klein, A., Koines, A., Seifert, M.: Generalized eigenfunctions for waves in inhomogeneous media. J. Funct. Anal. 190, 255-291 (2002) Zbl 1043.35097 MR 1895534

[KILS] Klein, A., Lacroix, J., Speis, A.: Localization for the Anderson model on a strip with singular potentials. J. Funct. Anal. 94, 135-155 (1990) Zbl 0780.60063 MR 1077548

[KlMP] Klein, A, Martinelli, F., Perez, J. F.: A rigorous replica trick approach to Anderson localization in one dimension. Comm. Math. Phys. 106 623-633 (1986) Zbl 0614.60098 MR 0860313 
[KlM] Klein. A., Molchanov, S.: Simplicity of eigenvalues in the Anderson model. J. Statist. Phys. 122, 95-99 (2006) Zbl 1152.82010 MR 2203783

[Klo1] Klopp, F.: Localization for semiclassical continuous random Schrödinger operators. II. The random displacement model. Helv. Phys. Acta 66, 810-841 (1993) Zbl 0820.60043 MR 1264047

[Klo2] Klopp, F.: Localization for continuous random Schrödinger operators. Comm. Math. Phys. 167, 553-569 (1995) Zbl 0820.60044 MR 1316760

[Klo3] Klopp, F.: Internal Lifshits tails for random perturbations of periodic Schrödinger operators. Duke Math. J. 98, 335-396 (1999) Zbl 1060.82509 MR 1845184

[Klo4] Klopp, F.: Weak disorder localization and Lifshitz tails. Comm. Math. Phys. 232, 125-155 (2002) Zbl 1034.82024 MR 1942859

[Klo5] Klopp F.: Weak disorder localization and Lifshitz tails: continuous Hamiltonians. Ann. Inst. H. Poincaré 3, 711-737 (2002) Zbl 1016.60095 MR 1933367

[KloLNS] Klopp F., Loss, M., Nakamura, S., Stolz, G.: Localization for the random displacement model. Duke Math. J., 161, No. 4, 587-621 (2012). Zbl pre06024992

[KloN] Klopp F., Nakamura, S.: Lifshitz tails for generalized alloy-type random Schrödinger operators. Anal. PDE 3, 409-426 (2010) Zbl 1226.35058 MR 2718259

[KuS] Kunz, H., Souillard, B.: Sur le spectre des opérateurs aux différences finies aléatoires. Comm. Math. Phys. 78, 201-246 (1980) Zbl 0449.60048 MR 0597748

[L] Lacroix, J.: Localisation pour l'opérateur de Schrödinger aléatoire dans un ruban. Ann. Inst. H. Poincaré Sect. A 40, 97-116 (1984) Z Zbl 0599.60062 MR 0745684

[LeMW] Leschke, H., Müller, P., Warzel, S.: A survey of rigorous results on random Schrödinger operators for amorphous solids. Markov Process. Related Fields 9, 729 760 (2003) Zbl 1204.82025 MR 2072253

[LiGP] Lifshits, I. M., Gredeskul, A. G., Pastur, L. A.: Introduction to the Theory of Disordered Systems. Wiley-Interscience, New York (1988) MR 1042095

[MS1] Martinelli, F., Scoppola, E.: Remark on the absence of absolutely continuous spectrum for $d$-dimensional Schrödinger operators with random potential for large disorder or low energy. Comm. Math. Phys. 97, 465-471 (1985) Zbl 0603.60060 MR 0778628

[MS2] Martinelli, F., Scoppola, E.: Introduction to the mathematical theory of Anderson localization. Riv. Nuovo Cimento 10, 1-90 (1987) MR 0924528

[PF] Pastur, L., Figotin, A.: Spectra of Random and Almost-Periodic Operators. Springer, Heidelberg (1992) Zbl 0752.47002 MR 1223779

[RS] Reed, M., Simon, B.: Methods of Modern Mathematical Physics, Vol. IV, Analysis of Operators. Academic Press (1978) Zbl 0401.47001 MR 0493421

[SVW] Shubin, C., Vakilian, R., Wolff, T.: Some harmonic analysis questions suggested by Anderson-Bernoulli models. Geom. Funct. Anal. 8, 932-964 (1998) Zbl 0920.42005 MR 1650106

[SiW] Simon, B., Wolff, T.: Singular continuum spectrum under rank one perturbations and localization for random Hamiltonians. Comm. Pure Appl. Math. 39, 75-90 (1986) Zbl 0609.47001 MR 0820340

[Sp] Spencer, T.: Localization for random and quasiperiodic potentials. J. Statist. Phys. 51, 1009-1019 (1988) Zbl 1086.82547 MR 0971043

[St] Stollmann, P.: Wegner estimates and localization for continuum Anderson models with some singular distributions. Arch. Math. (Basel) 75, 307-311 (2000) Zbl 1068.82523 MR 1786177

[Sto] Stolz, G.: Localization for random Schrödinger operators with Poisson potential. Ann. Inst. H. Poincaré Phys. Théor. 63, 297-314 (1995) Z Zbl 0843.60058 MR 1363537 
[U] Ueki, N.: Wegner estimates and localization for Gaussian random potentials. Publ. Res. Inst. Math. Sci. 40, 29-90 (2004) Zbl 1134.60354 MR 2030070

[W1] Wang, W.-M.: Microlocalization, percolation, and Anderson localization for the magnetic Schrödinger operator with a random potential. J. Funct. Anal. 146, 1-26 (1997) Zbl 0872.35137 MR 1446374

[W2] Wang, W.-M.: Localization and universality of Poisson statistics for the multidimensional Anderson model at weak disorder. Invent. Math. 146, 365-398 (2001) Zbl 1035.82023 MR 1865399

[We] Wegner, F.: Bounds on the density of states in disordered systems. Z. Phys. B 44, 9-15 (1981) MR 0639135

[Y] Yurinsky, V.: Sums and Gaussian Vectors. Lecture Notes in Math. 1617, Springer, Berlin (1995) Zbl 0846.60003 MR 1442713 